\title{
Patagonia's diverse but homogeneous early Paleocene forests: Angiosperm leaves from the Danian Salamanca and Peñas Coloradas formations, San Jorge Basin, Chubut, Argentina
}

\author{
Ari Iglesias, Peter Wilf, Elena Stiles, and Rebecca Wilf
}

\begin{abstract}
Early Paleocene macrofloras from the Southern Hemisphere are little known, despite their significance for understanding plant evolution, biogeography, and global variation in recovery after the end-Cretaceous extinction. As a foundation for systematic and paleoecological work, we describe 51 angiosperm leaf morphotypes from three distinct, precisely dated early to late Danian time intervals, using collections from the Salamanca and Peñas Coloradas formations in the San Jorge Basin, Chubut Province, Patagonia, Argentina. These rich floras were previously analyzed but with minimal descriptions. The assemblages comprise the first stratigraphically controlled and quantitatively collected floras for the early Paleocene of the Southern Hemisphere. Botanical affinities of the angiosperm morphotypes are not formally assigned here, but we informally associate some of them with families including Arecaceae, Fabaceae, Cunoniaceae, Lauraceae, Nothofagaceae, Rhamnaceae, and Rosaceae; in addition, leaves of Menispermaceae and other Rhamnaceae were formally described in previous work. Other families potentially present in these assemblages include Akaniaceae, Anacardiaceae, Apiaceae, Araceae, Bixaceae, Juglandaceae, Malvaceae, Sapindaceae, and Urticaceae. Remarkably, there is little floral turnover or change in dominance through the Danian floral sequence spanned by the studied localities, even among estuarine vs. continental depositional environments. This finding indicates a homogeneous, generalist, long-lived floral association following the K-Pg extinction, similar in these respects to many North American Danian floras. However, the richness of the Danian Patagonian floras, from paleolatitudes $>50$ degrees South, along with other lines of evidence from the region, suggests differences in the response of terrestrial ecosystems in southern South America to the terminal Cretaceous event from those of the Northern Hemisphere. The flora appears to be largely paleo-endemic in nature and shows several compositional links to the Eocene floras of Patagonia, emphasizing the importance of diversification within Patagonia after the end-Cretaceous event as a factor leading to the hyperdiverse Eocene regional floras.
\end{abstract}

Iglesias, Ari, Wilf, Peter, Stiles, Elena, and Wilf, Rebecca. 2021. Patagonia's diverse but homogeneous early Paleocene forests: Angiosperm leaves from the Danian Salamanca and Peñas Coloradas formations, San Jorge Basin, Chubut, Argentina. Palaeontologia Electronica, 24(1):a02. https://doi.org/10.26879/1124

palaeo-electronica.org/content/2021/3257-patagonia-danian-forests

Copyright: January 2021 Paleontological Society.

This is an open access article distributed under the terms of Attribution-NonCommercial-ShareAlike 4.0 International (CC BY-NC-SA 4.0 ), which permits users to copy and redistribute the material in any medium or format, provided it is not used for commercial purposes and the original author and source are credited, with indications if any changes are made. creativecommons.org/licenses/by-nc-sa/4.0/ 
Ari Iglesias. Instituto de Investigaciones en Biodiversidad y Ambiente INIBIOMA, CONICET-UNCO, San Carlos de Bariloche, 8400, Río Negro, Argentina. ari_iglesias@yahoo.com.ar (corresponding author) Peter Wilf. Department of Geosciences and Earth and Environmental Systems Institute, Pennsylvania State University, University Park, Pennsylvania 16802, USA. pwilf@psu.edu Elena Stiles. Department of Geosciences and Earth and Environmental Systems Institute, Pennsylvania State University; and Department of Biology and Burke Museum of Natural History, University of Washington, Seattle, Washington 98105, USA. estiles@uw.edu

Rebecca Wilf. The Arboretum at Penn State, Pennsylvania State University, University Park, Pennsylvania 16802, USA. rebeccawilf@gmail.com

Keywords: angiosperms; Danian; extinction/recovery; fossil leaves; Paleocene; South America

Submission: 20 August 2020. Acceptance: 11 December 2020.

\section{INTRODUCTION}

The Southern Hemisphere has produced few known macrofloras that can be reliably dated to the early Paleocene (Danian stage) (Vajda et al., 2001; Vajda and Raine, 2003; Vajda and McLoughlin, 2006; Pole and Vajda, 2009; Ferrow et al., 2011; Steinthorsdottir et al., 2016; Barreda et al., 2012; Clyde et al., 2014; Askin, 1988; Macphail, 1994). This paucity of information has impaired our understanding of floral recovery over a vast area of the planet following the globally disruptive end-Cretaceous extinction event.

The presence of Paleocene macrofloras in the Salamanca Formation of southern Chubut Province, Argentina, has long been known (Berry, $1937 a$ ), but for many decades they were the focus of few studies, and their precise ages remained uncertain (see Clyde et al., 2014). Most paleobotanical work from the Salamanca Formation had focused on the abundantly preserved, permineralized fossil wood and occasional fruits that so far represent relatively few, though significant, taxa of conifers and angiosperms (Petriella, 1972; Petriella and Archangelsky, 1975; Romero, 1968; Ragonese, 1980; Brea et al., 2005, 2007, 2008, 2011; Futey et al., 2012; Ruiz et al., 2017, 2020). More recently, several macrofloral compression assemblages from the Salamanca and overlying Peñas Coloradas formations were intensively collected in the vicinity of Sarmiento, southern Chubut, and found to be outstandingly preserved and diverse (Brea and Zucol, 2006; Iglesias, 2007; Iglesias et al., 2007; Raigemborn et al., 2009). These floras, dominated by fossil angiosperm leaves, are also now well-dated to the early and late Danian based on numerous new chronostratigraphic constraints (Table 1), and they have been paleoenvironmentally interpreted based on high- resolution stratigraphic and facies analyses (Clyde et al., 2014; Comer et al., 2015; Table 1).

The exquisitely preserved Salamanca and Peñas Coloradas compression floras have been the focus of several recent systematic and paleoecological studies, significantly increasing the understanding of early Paleocene floral composition and ecosystems in Patagonia. Among gymnosperms, the Salamanca Formation produced associated leafy branches, pollen cones, ovuliferous complexes, and seeds of the oldest known Agathis Salisbury, 1807 (Araucariaceae; Escapa et al., 2018); leafy branches with in situ cuticles of the oldest taxon attributable to the scale-leaved clade of Podocarpaceae (Andruchow-Colombo et al., 2018); and the oldest record of the podocarp genus Dacrycarpus (Endlicher) de Laubenfels, 1969 (Quiroga et al., 2016). Angiosperm taxa described from the Salamanca Formation include wood and an associated leaflet of Fabaceae Lindley, 1836 (Brea et al., 2008); associated flowers and leaves of the first fossil Rhamnaceae Jussieu, 1789, known from the Southern Hemisphere (Jud et al., 2017); flowers with in situ pollen of Tribe Schizomerieae Bradford and Barnes, 2001 (Cunoniaceae Brown, 1814 nom. cons., Jud et al., 2018a); and the oldest Menispermaceae Jussieu, 1789 nom. cons. endocarp, associated with a diagnostic leaf of the family (Jud et al., 2018b). In addition fertile sporophytes of the aquatic fern Azolla Lamarck, 1783 were recently reported (Hermsen et al., 2019). Insect-feeding damage on the fossil angiosperm and conifer leaves supported the first analyses of the effects of the Cretaceous-Paleogene (K-Pg) extinction on plant-insect food webs in the Southern Hemisphere (Donovan et al., 2016, $2018,2020)$. The leaf floras of Iglesias et al. (2007) have also been used in several other paleoecological and paleoclimatic studies (Labandeira et al., 
TABLE 1. Censused abundance of angiosperm leaf morphotypes per plant locality, organized by geomagnetic polarity chron (following Clyde, et al., 2014; Comer, et al., 2015; and Gradstein, et al., 2012). Fossiliferous quarries from the Palacio de los Loros locality (Salamanca Formation): Palacio de los Loros 1 (PL1), Palacio de los Loros 2 (PL2), and Palacio de los Loros 4 (PL4). Fossiliferous quarries from the Ormachea Petrified Forest Park (Salamanca Formation): Ormachea-1 (OR1), Ormachea-2 (OR2, including the subsite Cerro de las Palmeras CP), Cerro Solitario (CS), and Dromedary Hill (DR). Fossiliferous quarry from the Peñas Coloradas Formation: Las Flores oil field /La Campanita Ranch (LF).

\begin{tabular}{|c|c|c|c|c|c|c|c|c|c|c|}
\hline \multirow{2}{*}{$\begin{array}{c}\text { Leaf } \\
\text { Morphotype }\end{array}$} & \multirow{2}{*}{$\begin{array}{l}\text { Family } \\
\text { affinity }\end{array}$} & \multicolumn{4}{|c|}{ C29n } & \multicolumn{3}{|c|}{ C28n } & \multirow{2}{*}{$\begin{array}{c}\text { C27n } \\
\text { LF }\end{array}$} & \multirow{2}{*}{$\begin{array}{c}\text { Tot. } \\
8\end{array}$} \\
\hline & & OR2 & PL1 & PL4 & CS & OR1 & DR & PL2 & & \\
\hline SA001 & Akaniaceae? & - & 3 & - & - & 1 & - & 16 & - & 20 \\
\hline SA002 & unknown & - & 14 & - & - & - & - & 13 & - & 27 \\
\hline SA004 & Nothofagaceae & - & 69 & 4 & 2 & - & 3 & 21 & 93 & 192 \\
\hline SA005 & Cunoniaceae? & - & 12 & 2 & 1 & 4 & 1 & 10 & 4 & 34 \\
\hline SA008 & Rosaceae? & 3 & 53 & 34 & - & 8 & 1 & 12 & 4 & 115 \\
\hline SA009 & unknown & 2 & 208 & 43 & 4 & 1 & - & 256 & 28 & 542 \\
\hline SA010 & Lauraceae & 112 & 210 & 60 & 1 & 12 & 2 & 179 & 106 & 682 \\
\hline SA014 & unknown & 1 & 95 & 19 & - & - & - & 66 & 20 & 201 \\
\hline SA016 & unknown & - & 3 & - & - & 1 & 1 & 26 & 5 & 36 \\
\hline SA019 & unknown & 1 & 63 & 28 & - & 1 & - & 22 & 11 & 126 \\
\hline SA020 & Cunoniaceae & 2 & 4 & 8 & - & 3 & 1 & 129 & 13 & 160 \\
\hline SA032 & Arecaceae & 2 & 16 & - & - & - & - & 1 & 1 & 20 \\
\hline SA035 & Bixaceae? & - & 13 & 1 & - & - & - & 25 & 7 & 46 \\
\hline SA038 & unknown & 5 & - & - & - & - & - & - & - & 5 \\
\hline SA039 & unknown & - & 10 & 4 & - & - & - & - & 3 & 17 \\
\hline SA040 & unknown & - & 4 & - & - & - & - & 1 & - & 5 \\
\hline SA041 & Rhamnaceae? & - & 9 & 2 & - & - & - & 42 & - & 53 \\
\hline SA042 & Cunoniaceae & - & 25 & 7 & - & 3 & - & 11 & 11 & 57 \\
\hline SA043 & $\begin{array}{l}\text { Cunoniaceae? - } \\
\text { Sapindaceae? }\end{array}$ & 5 & 1 & 12 & 1 & 6 & 2 & 58 & - & 85 \\
\hline SA044 & Cunoniaceae & 3 & 63 & 14 & - & 2 & - & 15 & 13 & 110 \\
\hline SA045 & Fabaceae & 1 & 4 & - & - & - & - & - & - & 5 \\
\hline SA046 & Lauraceae & 1 & 56 & 25 & - & - & 2 & 9 & - & 93 \\
\hline SA047 & unknown & 1 & 18 & 2 & - & - & - & 38 & 15 & 74 \\
\hline SA048 & Rhamnaceae? & 1 & 105 & 22 & - & 1 & - & 8 & 19 & 156 \\
\hline SA049 & unknown & - & 17 & 22 & - & - & 1 & 285 & - & 325 \\
\hline SA050 & Anacardiaceae? & 1 & - & - & - & - & - & 9 & - & 10 \\
\hline SA051 & Urticaceae? & - & 2 & - & - & - & - & 5 & - & 7 \\
\hline SA052 & Sapindaceae? & - & 1 & - & - & - & - & - & - & 1 \\
\hline SA053 & unknown & 5 & 8 & 1 & - & - & - & 2 & - & 16 \\
\hline SA054 & Cunoniaceae? & - & - & - & - & - & - & 10 & - & 10 \\
\hline SA055 & unknown & - & - & - & - & - & - & 1 & 1 & 2 \\
\hline SA056 & unknown & 3 & 4 & 2 & - & - & - & 4 & 6 & 19 \\
\hline SA057 & unknown & - & - & - & - & - & - & 1 & - & 1 \\
\hline SA058 & Malvaceae? & - & 3 & - & - & - & - & 9 & 1 & 13 \\
\hline SA059 & unknown & - & - & - & - & - & - & 1 & 1 & 2 \\
\hline SA060 & Menispermaceae & - & 2 & - & - & - & - & - & - & 2 \\
\hline SA061 & Araceae? & 1 & - & - & - & - & - & 8 & - & 9 \\
\hline SA063 & unknown & 14 & - & - & - & - & - & - & - & 14 \\
\hline
\end{tabular}


TABLE 1 (continued).

\begin{tabular}{|c|c|c|c|c|c|c|c|c|c|c|}
\hline \multirow{2}{*}{$\begin{array}{l}\text { Leaf } \\
\text { Morphotype }\end{array}$} & \multirow{2}{*}{$\begin{array}{l}\text { Family } \\
\text { affinity }\end{array}$} & \multicolumn{4}{|c|}{ C29n } & \multicolumn{3}{|c|}{ C28n } & \multirow{2}{*}{$\begin{array}{c}\text { C27n } \\
\text { LF }\end{array}$} & \multirow{2}{*}{$\begin{array}{c}\text { Tot. } \\
8\end{array}$} \\
\hline & & OR2 & PL1 & PL4 & CS & OR1 & DR & PL2 & & \\
\hline SA065 & Arecaceae & 1 & - & - & - & - & - & - & - & 1 \\
\hline SA066 & unknown & 2 & - & - & - & - & - & - & 2 & 4 \\
\hline SA067 & unknown & 1 & - & - & - & - & - & - & - & 1 \\
\hline SA068 & unknown & - & - & - & - & - & - & - & 33 & 33 \\
\hline SA069 & Rhamnaceae? & - & - & - & - & - & - & - & 1 & 1 \\
\hline SA070 & Juglandaceae? & - & - & - & - & - & - & - & 17 & 17 \\
\hline SA072 & Arecaceae & $\begin{array}{c}\text { (4 at } \mathrm{CP} \\
\text { site) }\end{array}$ & - & - & - & - & - & - & - & 4 \\
\hline SA073 & unknown & - & - & - & - & - & - & - & 108 & 108 \\
\hline SA074 & unknown & - & - & - & - & - & - & - & 6 & 6 \\
\hline SA075 & unknown & - & - & - & - & - & - & - & 36 & 36 \\
\hline SA076 & unknown & - & - & - & - & - & - & - & 5 & 5 \\
\hline SA077 & unknown & - & - & - & - & - & - & - & 11 & 11 \\
\hline \multirow[t]{3}{*}{ SA078 } & unknown & - & - & - & - & - & - & - & 3 & 3 \\
\hline & \# specimens & 172 & 1095 & 312 & 9 & 43 & 14 & 1293 & 584 & 3522 \\
\hline & \# morphotypes & 22 & 30 & 21 & 5 & 12 & 9 & 32 & 30 & 51 \\
\hline
\end{tabular}

2007; Wing et al., 2009; Feild et al., 2011; Peppe et al., 2011, Hinojosa et al., 2011), including a related paper that is the first quantitative analysis of $\mathrm{K}-\mathrm{Pg}$ macrofloral turnover in Patagonia (Stiles et al., 2020).

Although the Danian Patagonian leaf floras have supported many advances in our understanding of early Paleocene floral diversity and composition in the region, the extensive angiosperm leaf collections have so far only been described in preliminary form and only from one site (Palacio de los Loros; Iglesias et al., 2007). These collections have since expanded in number of specimens and localities, and they now represent the most abundant and diverse Danian compression floras for the Southern Hemisphere.

Here, we take a critical step toward elevating our understanding of the informative Danian floras of Patagonia and their broader systematic, evolutionary, and biogeographic research potential by establishing a descriptive framework and inventory for the diverse morphotypes of angiosperm leaf compressions of the Salamanca and Peñas Coloradas formations. The descriptions presented here document the angiosperm leaf morphotypes and establish them as recognizable, vouchered entities across the large Danian collections from different sites and formations. This morphotype approach has already proven beneficial for associated angiosperm leaves and reproductive organs of Menispermaceae and Rhamnaceae from the floras studied here (e.g., Jud et al., 2017, 2018b) and, in general, in many other deposits around the world (e.g., Johnson et al., 1989). We briefly interpret the leaf morphotypes with regard to recovery from the end-Cretaceous extinction and floral turnover through the Danian of Patagonia; however, this issue is explored in subtantially more detail in a related paper (Stiles et al., 2020).

\section{GEOLOGICAL SETTING}

The fossil floras studied here come from the westernmost exposures of Paleocene strata in the north-central San Jorge Basin, southern Chubut Province, Argentina (Figure 1) at a paleolatitude of ca. $51^{\circ} \mathrm{S}$ as detailed in several recent papers (Iglesias et al., 2007; Clyde et al., 2014; Comer et al., 2015) and summarized here. The fossil sites (Figures 1-4; Table 1) are located in natural outcrops of two formations: the estuarine, early Danian Salamanca Formation; and the overlying, fluvio-volcanic, late Danian Peñas Coloradas Formation of the Río Chico Group. The chronostratigraphic framework for these formations in the study area is based on U-Pb and ${ }^{40} \mathrm{Ar}-39 \mathrm{Ar}$ geochronology; paleomagnetic stratigraphy; and biostratigraphy of foraminifera, dinocysts, calcareous nanoplankton, and palynomorphs (Clyde et al., 2014; Comer et al., 2015; Krause et al., 2017). This information, coupled with detailed facies analyses of the depositional environments of the fossil plant localities 


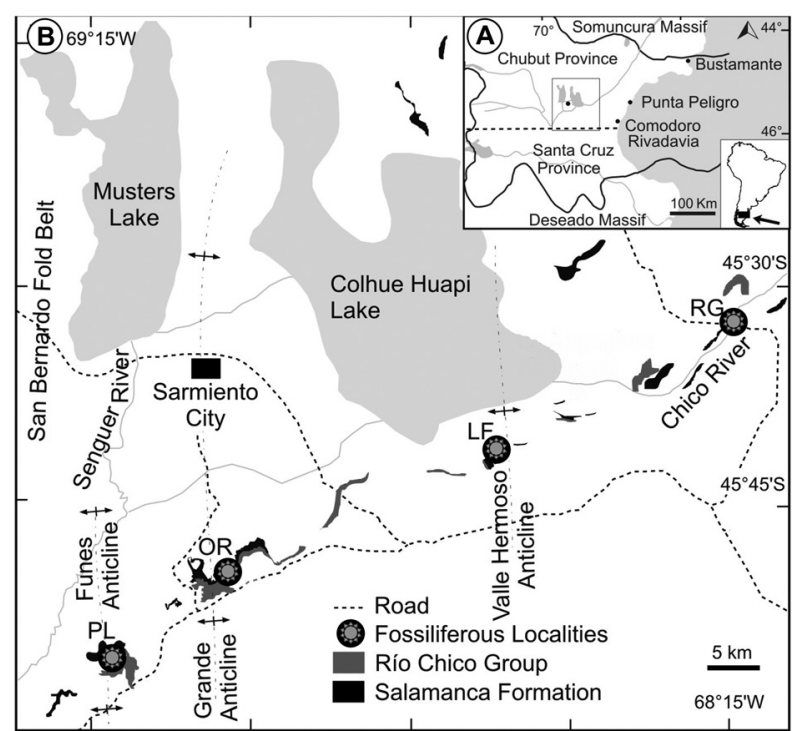

FIGURE 1. A, Regional map with early Paleocene outline of the San Jorge Basin (dark line; after Sylwan, 2001), with rectangular inset for study area (B); B, Salamanca Formation and Río Chico Group outcrops in the Sarmiento area and major geological structures. Fossil localities: OR, Ormachea Petrified Forest Park; PL, Palacio de los Loros; LF, Las Flores; RG, Rancho Grande.
(Comer et al., 2015), provides robust geological constraints on all the fossil quarries discussed in this paper (Table 1).

The bulk of the Salamanca Formation in the study area lies below an unconformity (interpreted as a sequence boundary) and is correlated to geomagnetic polarity Chron C29n (early Danian, 65.58-64.86 Ma per Gradstein et al., 2012; Clyde et al., 2014; Comer et al., 2015). The sediments above the sequence boundary are constrained to Chron C28n (early Danian, 64.67-63.49 Ma). The entire Salamanca package represents sedimentation from a paleo-Atlantic estuarine system transitioning in its upper section to fluvially influenced mudstones from landward tidal channels and eventually coastal swamps (Clyde et al., 2014; Comer et al., 2015). Some of the most landward fossiliferous strata derived from tidal channels contain quarry PL2 (Figure 2; Iglesias et al., 2007), preserving the most prolific and well-preserved fossil assemblage studied here. All Salamanca Formation leaf floras conspicuously preserve the trace fossil Cochlichnus Hitchcock, 1858, representing underwater burrows parallel to substrate. The cap-

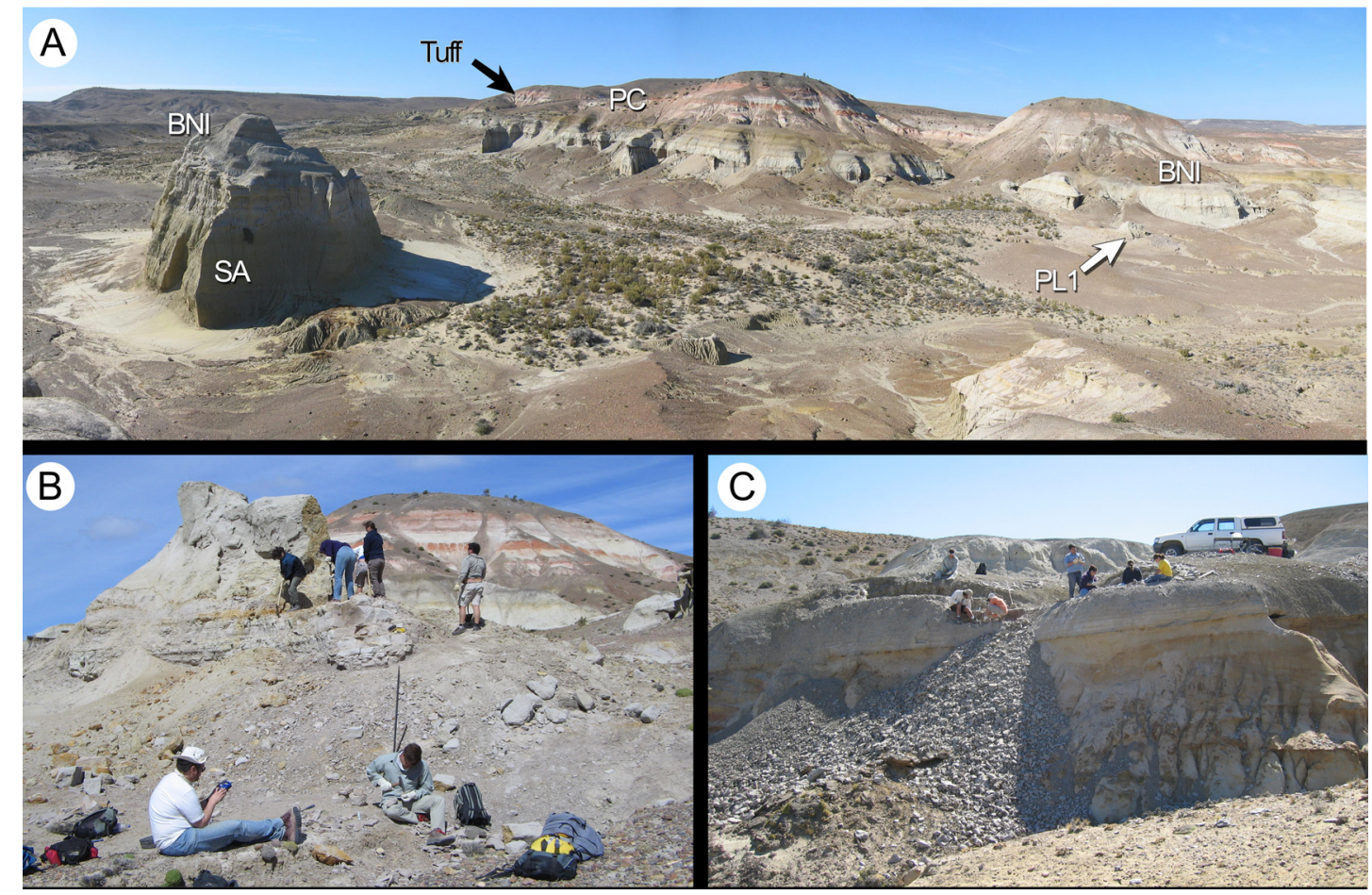

FIGURE 2. Paleocene fossil quarries at Palacio de los Loros (PL). A, General view of the Danian Salamanca (SA) and Peñas Coloradas (PC) formations, showing the distinctive butte at left that is home to large numbers of burrowing parrots (Cyanoliseus patagonus Vieillot, 1818); the locations of the PL1 quarry (white arrow) and the PL-1 tuff horizon (black arrow) dated in Clyde et al. (2014); and the Banco Negro Inferior (BNI) capping the Salamanca Formation. B, Quarry PL1 (assigned to Chron C29n, 65.58-64.86 Ma; Clyde et al., 2014; Comer et al., 2015). C, Quarry PL2 (Chron C28n, 64.67-63.49 Ma). 


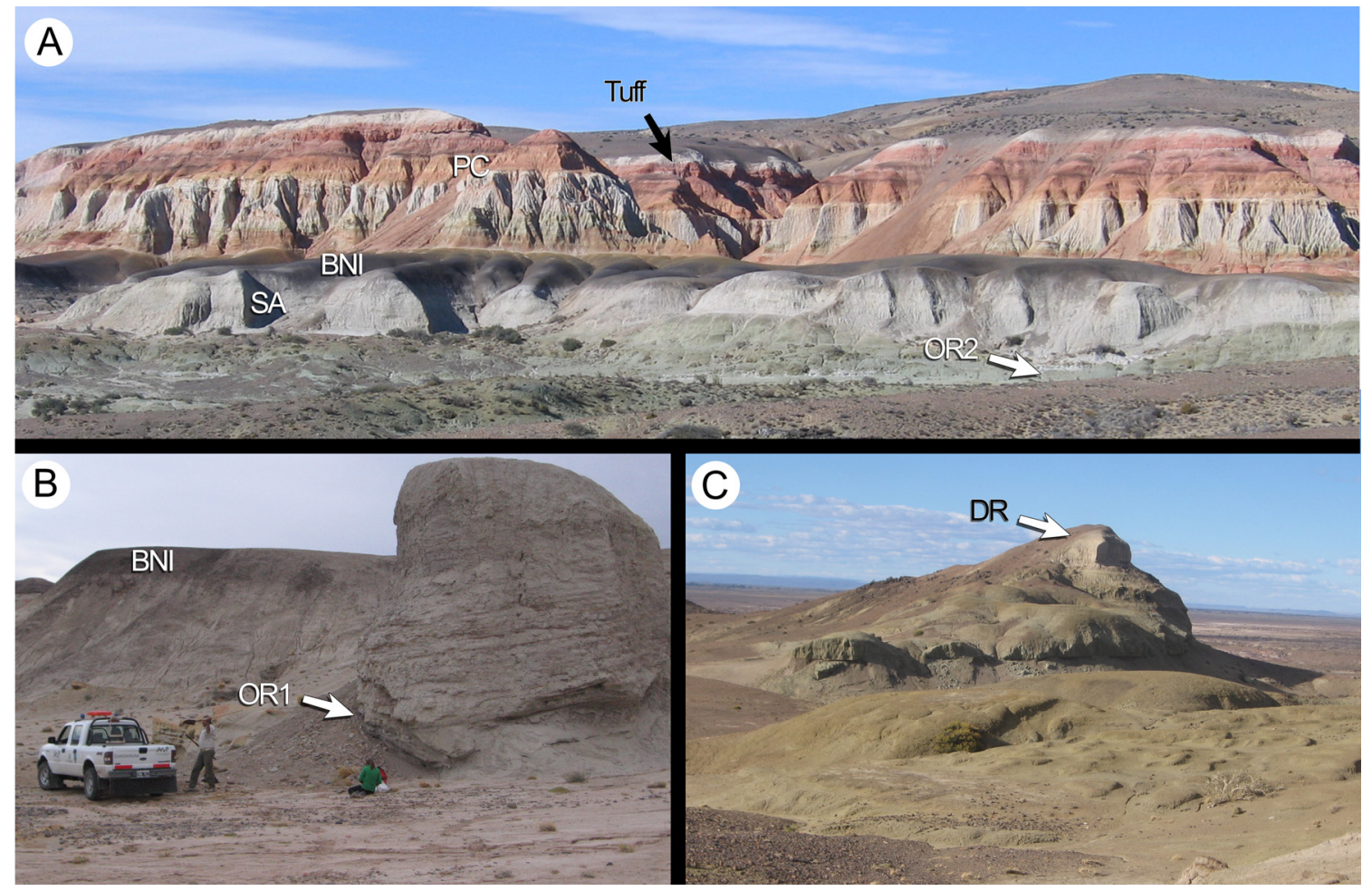

FIGURE 3. Strata exposed at Ormachea Petrified Forest Park. A, General view of the Danian Salamanca (SA) and Peñas Coloradas (PC) formations; white arrow marking OR2 quarry (Chron C29n), black arrow showing the white tuff horizon dated in Iglesias et al. (2007) and Clyde et al (2014). The prominent dark bed is the Banco Negro Inferior (BNI) at the top of the Salamanca Formation. Strata above the tuff include the Eocene Las Flores and Koluel Kaike formations. B, OR1 quarry (arrow, Chron C28n, 64.67-63.49 Ma) in heterolithic cross-bedded sandstones of the Salamanca Formation. C, Dromedary Hill, exposing part of the upper section of the Salamanca Formation (Chron C28n), arrow marking the DR fossil quarry. Scale ca. $7 \mathrm{~m}$.

ping unit of the Salamanca Formation is the Banco Negro Inferior (BNI, Figures 2A, 3A), a widespread coastal swamp deposit that is well known for preserving significant mammal and reptile occurrences in its eastern exposures at Punta Peligro along the modern coast (Figure 1; Bonaparte et al., 1993; Pascual et al., 2002; Sterli and de la Fuente, 2012). The BNI may be a time-transgressive unit, becoming younger toward the east where the fossil vertebrates are preserved (Krause et al., 2017). In our study area, the late Danian Peñas Coloradas Formation sits uncomformably above the BNI. The fossil flora studied here from the base of the Peñas Coloradas Formation at the Las Flores fossil-plant locality (LF, Figures 1,4 ) is assigned to Chron C27n (62.52-62.22 Ma; Clyde et al., 2014). Thus, the assemblages discussed here fall into three Danian time intervals, namely Chrons C29n (lower Salamanca floras), C28n (upper Salamanca floras), and C27n (Las Flores flora; Table 1).

Fossil floras of the Salamanca Formation discussed here come from the Palacio de los Loros $(\mathrm{PL})$ and Ormachea Petrified Forest Park (OR; also known as the Bosque Petrificado José Ormachea or the Monumento Provincial Bosque Petrificado Sarmiento) localities, as described previously (Iglesias 2007; Iglesias et al., 2007; Clyde et al., 2014; Comer et al., 2015; Table 1; Figures 1-3). Compressed fossil plants from Palacio de los Loros were first collected by A. Piatnitzky in the 1930s (see Feruglio 1949 page 316) and described by Berry (1937a; "Cerro Funes" flora), who published 11 species (Table 2) from a single site that we could not precisely relocate. The PL1 and PL2 macrofossil localities (quarries) from Palacio de los Loros were discovered and first studied by Iglesias et al. (2007), whereas the PL3, PL4, and PL5 quarries were both discovered and collected later (Clyde et al., 2014; Comer et al., 2015, Figure 4C). Among these more recent collections, the PL4 flora is the best preserved and was quantitatively collected (i.e., all identifiable material was collected; Table 1). Quarry PL2 is very fine-grained and also preserves many delicate flowers (Jud et al., 2018a), other plant reproductive material (Escapa et al., 2018; Jud et al., 2018b), leafy branches 


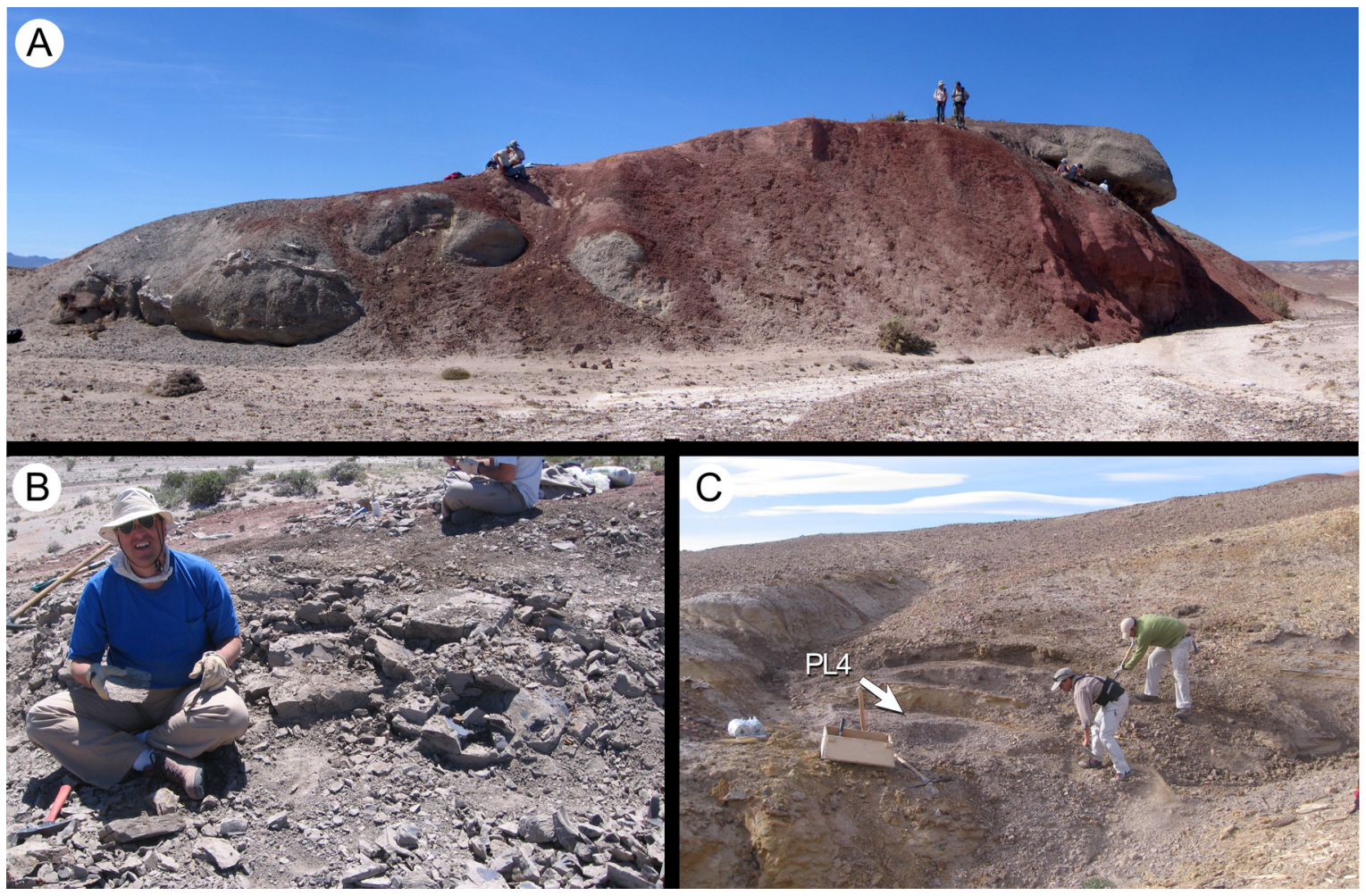

FIGURE 4. A-B, Las Flores oil field locality (La Campanita Ranch), Peñas Coloradas Formation. A, General view of coarse, continental fluvial channel deposits near the fossil locality, basal Peñas Coloradas Formation. B, Las Flores (LF) fossil quarry (Chron C27n, late Danian, 62.52-62.22 Ma). C, Palacio de los Loros PL4 quarry, Salamanca Formation (Chron C29n, early Danian, 65.58-64.86 Ma), arrow marking the fossiliferous level.

(Andruchow-Colombo et al., 2018), feathers (Tambussi and Degrange, 2013), and unpublished insect wings and bivalves. We note that the Rancho Grande flora, from a very different inner-shelf depositional environment of the Salamanca Formation assigned to Chron C29n (early Danian, 65.58-64.86 Ma, Clyde et al., 2014; Comer et al., 2015), is under separate study (e.g., Jud et al., 2017) and not covered here.

Fossil floras from OR were found at several quarries in the Salamanca Formation (Table 1): Ormachea-1 (OR1), Ormachea-2 (OR2, including the subsite Cerro de las Palmeras CP), Cerro Solitario (CS), and Dromedary Hill (DR) (Iglesias, 2007; Clyde et al., 2014; Comer et al., 2015). Quarry OR2 is the best preserved local assemblage, found in heterolithic cross-bedded sandstones correlated to Chron C29n along with the CS quarry (early Danian, 65.58-64.86 Ma, Clyde et al., 2014; Comer et al., 2015, Figure 3A); quarry CP, located in the lower Salamanca Formation, appears to be of similar age. Fossil quarries OR1 and DR are all composed of heterolithic cross-bedded sandstones from Chron C28n (early Danian,
64.67-63.49 Ma, Clyde et al., 2014; Comer et al., 2015, Figure 3B-C).

The late Danian (Chron C27n, 62.52-62.22 $\mathrm{Ma}$ ) flora was collected and field-censused at the Las Flores oil field quarry (LF) paleobotanical locality, at the La Campanita Ranch (Figure 1), corresponding to the base of the Peñas Coloradas Formation (Figure 4A-B). This site is geographically close to -but contains strata much older than - the overlying Eocene to Neogene sequence of fossil vertebrate localities along the Gran Barranca from the Las Flores and Sarmiento formations (e.g., Ameghino, 1906; Dunn et al., 2013; Woodburne et al., 2014; Krause et al., 2017). The LF flora occurs in fine-to-coarse sand channel-fill facies of a mixed-load fluvial system, containing a high proportion of volcanic clasts (Iglesias, 2007; Legarreta and Uliana, 1994; Raigemborn, 2005, 2006; Raigemborn et al., 2014; Comer et al., 2015). At the LF plant locality, Clyde et al. (2014) recorded terrestrial palynomorphs and a small number of dynocysts, indicating minor marine influence with low salinity. The only previously studied fossil plants from the Peñas Coloradas Formation include a few specimens of cunoniaceous flowers 
TABLE 2. Berry (1937a) type specimens from the Cerro Funes locality (Palacio de los Loros, Salamanca Formation), correlated to the leaf morphotypes in this work. USNM: Smithsonian National Museum of Natural History, Washington, D.C.

\begin{tabular}{|c|c|c|c|c|c|}
\hline \multirow[b]{2}{*}{ USNM \# } & \multicolumn{2}{|l|}{ Berry 1937a type collection } & \multirow[b]{2}{*}{ Figure } & \multirow[t]{2}{*}{$\begin{array}{l}\text { Morphotype } \\
\text { in this work }\end{array}$} & \multirow[t]{2}{*}{$\begin{array}{c}\text { Figures in this } \\
\text { work }\end{array}$} \\
\hline & Species & Plate & & & \\
\hline 201953 & Banaraphyllum ovatum & IX & 1 & SA048 & $27 \mathrm{D}, 28 \mathrm{~A}$ \\
\hline 201955 & Cissites patagonica & VII & 1 & SA009 & $10 \mathrm{~N}$ \\
\hline 201956a & Cissites patagonica & VII & 2 & SA009 & $100,10 P$ \\
\hline 201956b & Cissites patagonica & VII & 2 & SA009 & - \\
\hline 201957 & Cissites patagonica & VII & 3 & SA009 & $10 Q$ \\
\hline 201959 & "Cissites patagonica" & IX & 5 & SA073 & $36 \mathrm{C}$ \\
\hline 208520 & Cryptocaryoides maria-santisimensis & $\mathrm{VI}$ & 3 & SA010 & - \\
\hline 208521 & Cryptocaryoides maria-santisimensis & $\mathrm{VI}$ & 3 & SA010 & 12B \\
\hline 208522 & "Dryophyllum australis" & VI & 5 & SA042 & $7 \mathrm{~A}$ \\
\hline $208523 a$ & "Dryophyllum" australis & $\mathrm{VI}$ & 4 & SA004 & $7 \mathrm{~B}$ \\
\hline $208523 b$ & "Dryophyllum" australis & - & - & SA004 & - \\
\hline 201960 & Fagophyllum duseni & V & 1 & SA042 & $16 \mathrm{~L}$ \\
\hline 201961a & Fagophyllum duseni & V & 2 & SA042 & $16 \mathrm{M}$ \\
\hline $201961 b$ & Fagophyllum duseni & - & - & SA042 & - \\
\hline 208530 & Laurophyllum chubutensis & IX & 3 & SA046 & $26 \mathrm{~F}$ \\
\hline 208531 & Laurophyllum chubutensis & IX & 4 & SA046? & - \\
\hline 201965 & Laurophyllum piatnitzkyi & VI & 1 & SA010 & - \\
\hline 201966 & Laurophyllum piatnitzkyi & VI & 2 & SA010 & $12 \mathrm{~A}$ \\
\hline 201962 & "Myrica premira" & V & 3 & SA020 & $29 \mathrm{C}$ \\
\hline 201963 & "Myrica" premira & V & 4 & SA049 & $29 A, 29 B$ \\
\hline 201964 & "Paranymphaea" aristolochiaformis & V & 5 & SA039 & $21 \mathrm{~A}, 22 \mathrm{~A}$ \\
\hline $208524 a$ & "Sterculia" acuminataloba & VIII & 1 & SA014 & $14 \mathrm{~A}$ \\
\hline $208524 b$ & "Sterculia" acuminataloba & - & - & SA014 & - \\
\hline 208525 & "Sterculia acuminataloba" & VIII & 2 & SA058 & 321 \\
\hline 208526 & "Sterculia" acuminataloba & VIII & 3 & SA014 & 14B \\
\hline 208527 & "Sterculia" acuminataloba & VIII & 4 & SA014 & - \\
\hline 208528 & "Sterculia acuminataloba" & VIII & 5 & SA019 & 171 \\
\hline 208529 & Ziziphus chubutensis & IX & 2 & SA048 & $27 \mathrm{C}, 28 \mathrm{~B}$ \\
\hline
\end{tabular}

(Jud et al. 2018a), a single species of petrified wood assigned to Cordioxylon Awasthi, 1984 (Boraginaceae Adanson, 1763), and dispersed phytoliths from sites to the east, along the modern coast (Brea and Zucol, 2006; Raigemborn et al., 2009).

\section{METHODS}

The small type collection of 11 species from Palacio de los Loros (Berry, 1937a; Table 2), housed at the Smithsonian National Museum of Natural History, Washington, DC (acronym USNM), was examined at USNM and in the Penn State Paleobotany Laboratory.

We made the collections studied here over several field seasons (2003-2012) from bench quarries, using standard field reconnaissance to find the sites, hand tools, and occasionally a portable pneumatic hammer. The fossiliferous localities (Table 1) were collected selectively at first, but those that showed abundant and well-preserved material were also quantitatively censused (all identifiable material collected and brought to the museum for tallying) and field-labeled with unique field numbers (Iglesias et al., 2007). Census sam- 
ples included 1,093 angiosperm leaves (or leaflets for compound leaves) for quarry PL1; 1,189 for PL2; 380 for PL4; 168 for OR2; and 597 for LF, for a total of 3,522 leaf-fossil specimens (Table 1) collected and accessioned at Museo Paleontológico Egidio Feruglio (MEF, Trelew, Argentina, repository acronym MPEF-Pb). In addition to the unique field number labeled for each specimen, many received a formal MPEF-Pb catalog number as well (Appen$\operatorname{dix} 1)$.

Fossils were prepared with needles and air tools at the Museo de Ciencias Naturales de La Plata (MLP, La Plata, Argentina) and MEF; MEF is the repository for all collections described here, other than the Berry (1937a) types at USNM. Specimens were observed using a Wild M5 stereoscope at MLP and a Nikon SMZ-1000 stereoscope at MEF, both with camera lucida attachments for drawings. All photography was done using a Nikon Coolpix 8800 digital camera, connected to a stereoscope when higher magnification was required.

We used standard terminology for leaf architectural descriptions (Hickey, 1973, 1979; Hickey and Wolfe, 1975; Ash et al., 1999; Ellis et al., 2009). Foliar rank is indicated if lower than $3 r$, to emphasize the visual distinctiveness of the morphotypes with relatively poor vein organization (Hickey, 1971; Green et al., 2014; Little et al., 2014). Sinus-bracing veins are used in the sense of Upchurch and Dilcher (1990) for lobed leaf forms. The term "leaf texture" (per Hickey, 1973) is used here, not to infer features of the once-living leaf, but to indicate directly observed variation in leaf preservation, resulting from some combination of original leaf properties and taphonomic processes, that we found useful in identification. When preservation permitted, vein density was measured based on standard techniques and is given as previously reported for most of these species in Feild et al. (2011).

The leaves were divided into morphotypes based on shared leaf architectural characters, following the procedures of Johnson et al. (1989) and Ash et al. (1999) and a conservative, "lumping" approach. Morphotyping is a well-established parataxonomic method that is widely used as an organizational step for investigating large, diverse, and newly collected fossil leaf floras for which much taxonomic work remains to be done, as is the case here. In practice, morphotypes may or may not correspond to preliminary approximations of biological species, but here we view each one as a likely species equivalent. Illustrating the value of the method, two Salamanca Formation angio- sperm leaf morphotypes have already advanced to formally described botanical entities (Jud et al., 2017, 2018b), as well as several fern and conifer morphotypes (see Introduction). Each morphotype is designated with the two-letter prefix "SA", plus a unique three-digit number, and is assigned a single exemplar and one or more accessory exemplar specimens to provide a reference set and potential future type specimens. The informal exemplars are chosen entirely from our field collections for comparative purposes, and in some instances they are listed in addition to previous formal type specimens of the same entity (Berry, 1937a).

Morphotypes are simply presented as assigned in numerical, not systematic order, first for non-monocot (Figures 5-40) and then for monocot angiosperms (Figures 40-41). We note that there are several "missing" morphotype numbers that variously pertain to combined or to non-angiosperm morphotypes, some of these now published formally (e.g., SA018 as Agathis immortalis Escapa, Iglesias, Wilf, Catalano, Carballo-Ortiz and Cúneo, 2018; SA062 as Dacrycarpus sp. in Quiroga et al., 2016, figure 1a-1c; and SA071 as Kirketapel salamanquensis Andruchow-Colombo, Escapa, Carpenter, Iglesias, Arbazua and Wilf, 2018), and several not yet published, including ferns (see Iglesias, 2007). To avoid disrupting the museum catalog and to maintain consistency with prior and ongoing work (e.g., Iglesias et al., 2007; Donovan et al., 2016, 2018; Stiles et al., 2020), we have left the numbering gaps and not changed any morphotype numbers. A complete inventory of referred specimens for all morphotypes is provided in Appendix 1 and an identification key to the morphotypes in Appendix 2. We note in the descriptions below all morphotypes that appear to represent the same entities as the Berry (1937a) types (see also Table 2).

All material that is cited and figured here (Figures 5-41) is assigned repository numbers (acronyms USNM and MPEF-Pb), whereas material from the large general collection is mostly listed by field number (Appendix 1). Table 1 summarizes fossil quarries by time interval (geomagnetic polarity chron based on Clyde et al., 2014 and Comer et al., 2015) and lists morphotype occurrences and relative abundances by locality. Possible botanical affinities for some of the morphotypes are indicated for general interest and briefly discussed, but we stress that the suggested affinities are still preliminary pending further investigation, and the focus here is on establishing descriptive categories and a large set of voucher specimens as a robust founda- 
tion for improved understanding of the Danian fossil floras of Patagonia, including formal systematic work. Preliminary taxonomic discussions of many of the angiosperm leaf morphotypes appeared in the lead author's Ph.D. thesis (Iglesias, 2007), and systematic studies of individual leaf species are the topics of separate investigations (Jud et al., 2017, 2018a, 2018b).

\section{NON-MONOCOT LEAF MORPHOTYPES}

\section{Morphotype SA001}

Affinity. Akaniaceae? Stapf, 1912 nom. cons. Exemplar. MPEF-PB-2020 from quarry PL2 (Figure $5 \mathrm{~B}, 5 \mathrm{H}$ )

\section{Figure 5}

Description. Blade symmetric, size microphyll, mean length $7.1(5.7-8.5) \mathrm{cm}$, mean width 2.4 (1.5$3.8) \mathrm{cm}$. Length:width ratio $3.7: 1$ (3.6-3.8:1). Margin dentate. Petiole/petiolule insertion marginal, length $>1.6 \mathrm{~cm}$, disposed at an angle to the main blade axis (Figure 5B, 5G-H). Blade shape ovate. Texture chartaceous. Base shape decurrent, angle acute (Figure 5B). Apex shape straight, angle acute (Figure 5C-D). Primary venation pinnate, primary stout, straight in course, tapering apically (Figure 5D). Basal veins three. Agrophic veins absent. Secondary venation semicraspedodromous, thin, course slightly curved, joining supradjacent secondaries via successive loops and nearperpendicular junctions. Spacing between secondaries uniform and subopposite. Secondaries decurrent on midvein (Figure 5A). Perimarginal venation a thick fimbrial vein, to $0.2 \mathrm{~mm}$ in width, thickening the margin and presumably stiffening the teeth (Figure 5J). Intersecondary veins thin, perpendicular to midvein, less than half of the subjacent secondary in length, becoming perpendicular to subjacent secondary in distal course, frequency one to less than one per secondary (Figure 5G). Intercostal tertiary venation random reticulate to weakly percurrent, course convex or straight, obtuse to midvein (Figure 5F). Epimedial tertiary venation mixed percurrent, course obtuse to midvein and approximately perpendicular to secondaries (Figure 5G). Fourth order veins nearly as thick in gauge as tertiaries, regular polygonal reticulate. Fifth order veins regular polygonal reticulate, tending to opposite percurrent (Figure 5F). Areoles polygonal, size medium (0.3-1 $\mathrm{mm}$ width), poorly developed, randomly oriented. Idioblasts (preserved as dark dots) common (Figure 5E). Teeth large, variable in size, simple, spinose, some recurved towards apex, length to $2 \mathrm{~mm}$, width to 2 $\mathrm{mm}$, absent near leaf base and best developed in medial to distal portions of the blade. Tooth shape concave/convex, concave/concave, concave/flexuous, or straight/convex; sinuses rounded (Figure $5 F)$. Tooth spacing uniform, up to two-three teeth per secondary vein and up to four teeth per centimeter, absent at leaf base (Figure 5A-B, 5D). Tooth venation originates from an exterior tertiary branch derived from a secondary loop. Principal vein basally deflected or running near the tooth basal flank, course slightly curved (Figure 5F).

Observations. Morphotype SA001 has a consistently angled petiole/petiolule that may indicate derivation from a compound leaf. Similarities to Akania Hooker, 1862 [in Bentham and Hooker, 1862] (Akaniaceae) include non-glandular, spinose teeth, secondaries that join at right angles, intersecondaries reaching brochidodromous arches, tooth and sinus venation with a principal vein running along the tooth basal flank and originating from a tertiary branch that bifurcates at the tooth base at an acute angle, and an intramarginal vein supplying accessory tooth venation. The living $A$. bidwillii (Hogg) Mabberley, 1989 (east Australia), differs from morphotype SA001 in having oblong, elongated leaflets, poorly developed secondary vein loops, and teeth that are more and irregularly spaced. The family is represented in the fossil record of Patagonia by two Eocene leaf species: Akania americana Romero and Hickey, 1976, and A. patagonica Gandolfo, Dibbern, and Romero, 1988, both from the early Eocene Laguna del Hunco flora (Romero and Hickey, 1976; Gandolfo et al., 1988; Wilf et al., 2005), and a Miocene wood (Brea et al., 2018). The two fossil leaf species share with morphotype SA001 the length:width ratio, acute base, toothed margin except at the base, angled petiole (petiolule), tooth characters and venation, fimbrial vein, and secondary venation. Morphotype SA001 is similar to the Eocene species but with a more ovate shape and a cuneate/decurrent base. Characters that in combination easily differentiate this morphotype from all others (see key in supplementary Appendix 2) include: blade ovate; secondary veins semicraspedodromous; teeth simple, recurved, spinose; basal portion of blade untoothed; and fimbrial vein present.

\section{Morphotype SA002}

Affinity. Unknown.

Exemplar. MPEF-PB-2021 from quarry PL2 (Figure $6 \mathrm{~A}, 6 \mathrm{~F}, 6 \mathrm{H}-\mathrm{I})$.

Figure 6 

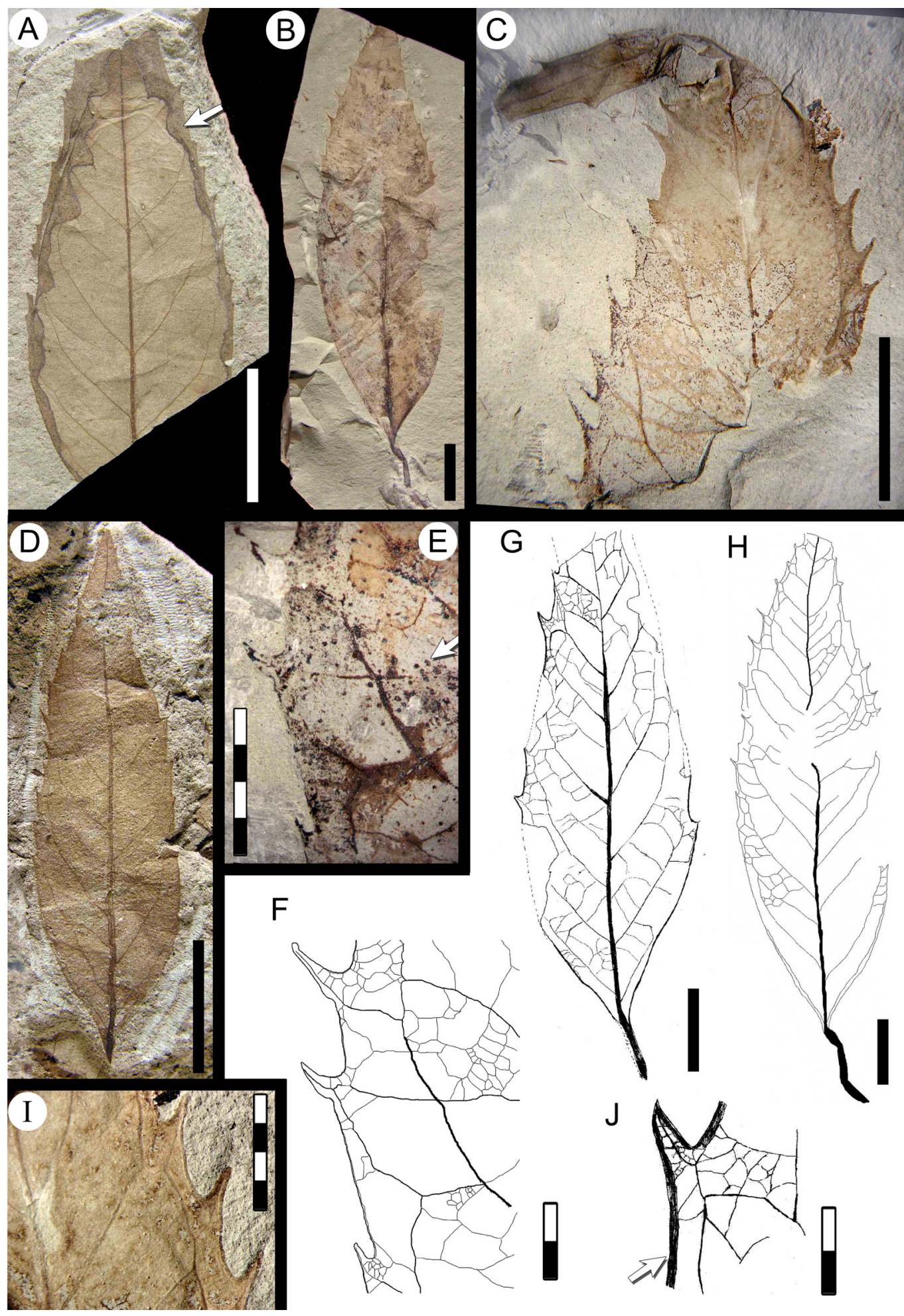

FIGURE 5. Morphotype SA001 (Akaniaceae?). A, MPEF-Pb-9000, note insect damage at the leaf margin (arrow; see also Labandeira et al., 2007 p.15, figure DT114); B, MPEF-Pb-2020 (exemplar, see also Figure 5H); C, MPEF-Pb9001; D, MPEF-Pb-4000; E, detail of tooth venation in Figure 5B, note darkly preserved idioblasts (arrow); F, digital overlay drawing (DOD) of teeth and associated venation of specimen in Figure 5E; G, camera lucida drawing (CLD) of leaf venation of MPEF-Pb-9002; H, DOD of leaf venation of specimen in Figure 5B (exemplar); I, tooth detail of specimen in Figure 5C; J, CLD of tooth venation in Figure 5G; note the thick fimbrial vein (arrow). Single-color scale bars equal $10 \mathrm{~mm}$; grid scales equal one millimeter (per rectangle). 


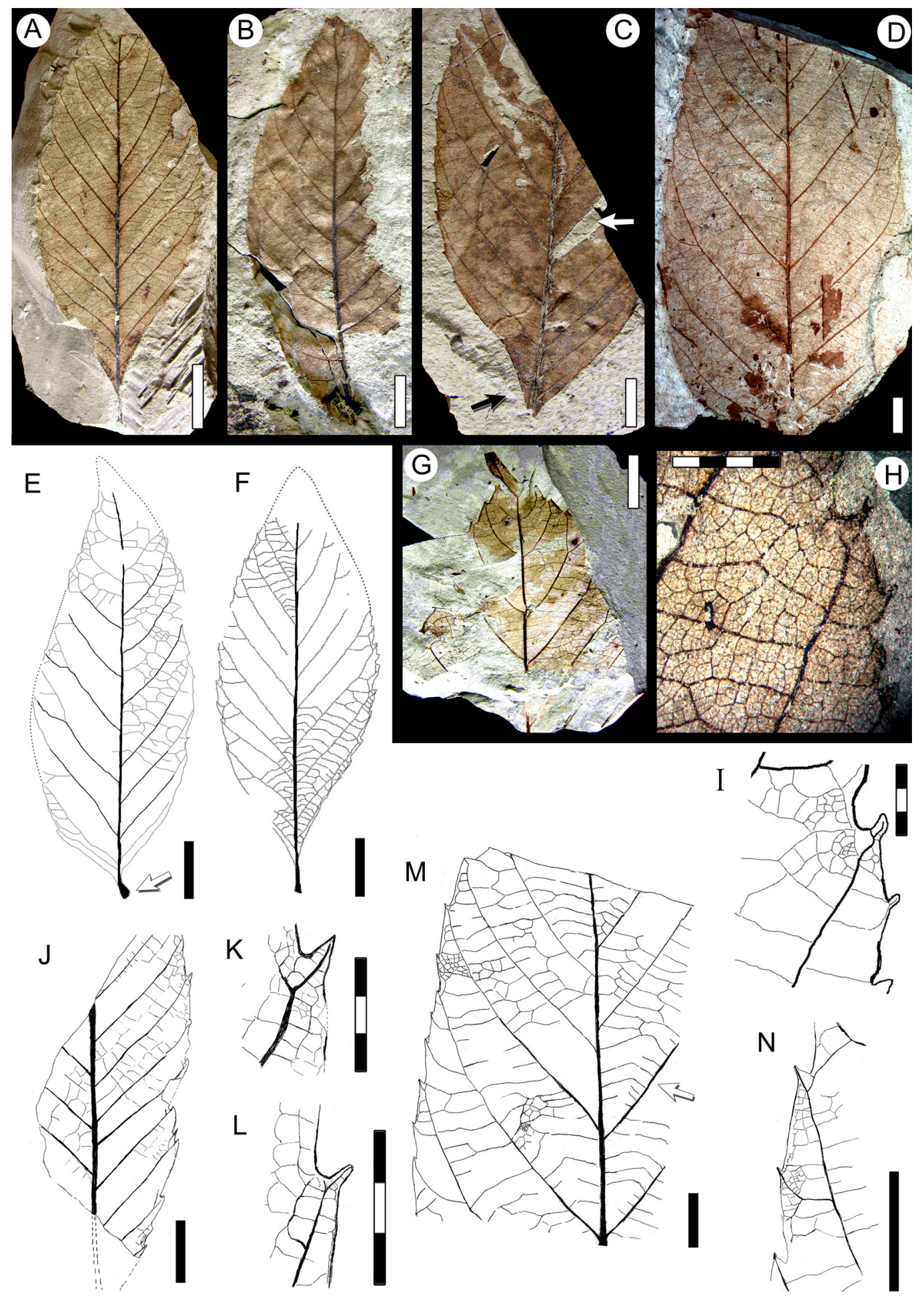

FIGURE 6. Morphotype SA002. A, MPEF-Pb-2021 (exemplar, see also Figure 6F); B, MPEF-Pb-4007; C, MPEF-Pb4010 (white arrow, asymmetric base), note insect damage (black arrow, DT26; also see Donovan et al., 2018 figure 9.16); D, MPEF-Pb-9004; E, digital overlay drawing (DOD) of leaf venation in Figure 6C (arrow, swollen petiole base; see also Figure 31F); F, DOD of leaf venation of specimen in Figure 6.A (exemplar); G, apex in MPEF-Pb-9005; $\mathrm{H}$, tooth detail of specimen in Figure 6.A; I, DOD of tooth venation of specimen in Figure $6 \mathrm{H}$; J, camera lucida drawing (CLD) of MPEF-Pb-9006; K, medial tooth venation of specimen in Figure 6J; L, basal tooth venation of specimen in Figure 6J; M, CLD of leaf venation of specimen in Figure 6D (arrow, chevroned epimedial tertiaries); N, tooth venation of specimen in Figure 6M. Single-color scale bars equal $10 \mathrm{~mm}$; grid scales equal one millimeter (per rectangle). 
Description. Blade simple, symmetrical, base asymmetrical (Figure 6C, 6E-F); size microphyll to mesophyll, most frequently notophyll; mean length $10.0(5.3-15.0) \mathrm{cm}$, mean width $4.5(3.1-6.0) \mathrm{cm}$. Length:width ratio 1.5:1 (1.1-1.9:1). Margin serrate. Petiole wide, insertion marginal, base swollen (Figure $6 \mathrm{E}$ ), length to $1 \mathrm{~cm}$. Shape slightly ovate to elliptic. Apex shape convex, angle acute (Figure $6 \mathrm{E}$ ). Base shape decurrent, angle acute (Figure $6 \mathrm{C}, 6 \mathrm{~F})$. Primary venation pinnate, primary vein straight and moderately thick. Basal veins one. Secondary venation craspedodromous, basally and apically crowded (Figure 6A-D), spacing irregular, characteristically forking near the margins (Figure 6E-F, 6K-L). Secondary veins moderate in thickness, arising at moderate angles $\left(54^{\circ}-65^{\circ}\right)$, course somewhat curved toward the margin. Perimarginal venation a well developed fimbrial vein (Figure 6K-L, 6H-I). Agrophic and intersecondary veins absent. Intercostal tertiary venation mixed percurrent or opposite percurrent (Figure 6M). Epimedial tertiary venation mixed opposite-alternate weakly percurrent to reticulate; course convex, somewhat chevroned near the primary (Figure $6 \mathrm{M})$; angle perpendicular to obtuse to primary vein. Fourth order veins random reticulate. Fifth order veins random reticulate, forming pentagonal or rectangular areoles of $0.4 \mathrm{~mm}$ width (Figure $6 \mathrm{H}-\mathrm{I}$, $6 \mathrm{M})$. Freely ending veinlets (FEVs) one- or twobranched (Figure $6 \mathrm{H}$ ). Marginal ultimate venation looped. Teeth simple, occasionally spinose, length to $0.5 \mathrm{~mm}$, width to $0.3 \mathrm{~mm}$ (Figure 6I, 6K-L, 6N). Tooth shape concave/convex, concave/straight, straight/convex, or flexuous/straight; sinuses rounded, sometimes angular (Figure 6I, 6N). Tooth spacing uniform, two-three per centimeter or secondary vein (Figure 6M). Tooth venation supplied by one of the branches of the forked secondary vein entering the tooth medially, or by a secondary branch that forks at the tooth base, generating an apical branch to the supradjacent sinus (Figure 6I, $6 \mathrm{~K}-\mathrm{L}, 6 \mathrm{~N}$ ). Medial vein slightly curved toward the tooth apex, occasionally projected out of the margin as a spine.

Observations. Morphotype SA002 occurs at low abundance and is known from quarries PL1 and PL2 (Salamanca Formation, Table 1). Its distinguishing characters include: blade elliptic to weakly ovate, secondary veins basally and apically crowded, tertiary veins somewhat chevroned near the primary vein, fimbrial vein well developed, and teeth regularly spaced (see key in supplementary Appendix 2).

\section{Morphotype SA004}

Equivalent. "Dryophyllum" australis Berry, 1937 in part, syntype USNM-208523 (Berry, 1937a, plate VI, figure 5; here Figure 7B) only (Table 2); syntype USNM-208522 excluded (see morphotype SA042).

Affinity. Nothofagaceae Kuprianova, 1962.

Exemplar. MPEF-PB-2022 from quarry PL2 (Figure 7C).

Accessory exemplar. MPEF-PB-2053 from quarry PL2 (Figure 7D-E).

Figure 7B-M

Description. Blade symmetric or weakly asymmetric; size notophyll to mesophyll, generally microphyll; mean length $7.6(3.5-11) \mathrm{cm}$, mean width 4.4 $(2.2-8) \mathrm{cm}$, length:width ratio 1.8:1 (1.5-2:1). Margin serrate, often not well preserved (Figure 7F, $7 \mathrm{~K})$. Petiole insertion marginal, base sometimes swollen, length to $1 \mathrm{~cm}$, width to $1.5 \mathrm{~mm}$. Texture membranaceous. Shape ovate (Figure 7C, 7G). Apex shape convex or weakly concave, angle acute. Base shape convex, rarely rounded; angle obtuse. Primary venation pinnate, primary vein thinner near middle of blade and dichotomizing at the apex, course sinuous, occasionally deflected at secondary junctions (Figure 7B, 7F, 7K). Agrophic veins present, simple or compound (Figure 7H, 7LM). Secondary venation craspedodromous; veins in 10-12 pairs; width moderate; opposite or subopposite to midvein; angle acute and uniform or slightly increasing toward the base (Figure $7 \mathrm{H}$ ), often asymmetric on either side of primary (Figure $7 \mathrm{C}$ ); secondary vein course straight, then curving smoothly towards the margin and generating two or three curving basal branches that enter minor teeth or their supradjacent sinuses (Figure 7E, 7H). Perimarginal and intersecondary veins absent. Intercostal tertiary venation weak, mixed oppositealternate percurrent; densely spaced; course very straight; angle uniform, sometimes increasing basally. Epimedial tertiary venation opposite percurrent, course curved, perpendicular or obtuse to midvein (Figure $7 \mathrm{~J}$ ). Fourth order and fifth order veins regular polygonal reticulate (Figure 7E). Areolation moderately developed. Freely ending veinlets not observed. Vein density range 7.59-11.69 $\mathrm{mm} / \mathrm{mm}^{2}$. Marginal ultimate venation looped, loops sometimes incomplete (Figure 7E). Teeth simple or compound (Figure 7E, 7I), length to $5 \mathrm{~mm}$, may be simple or appear in two orders; tooth apex simple or shortly mucronate. Tooth shape convex/convex for simple teeth, convex/convex or flexuous/flexuous for compound teeth, which have two-three minor teeth on the basal flank and one-two on the 

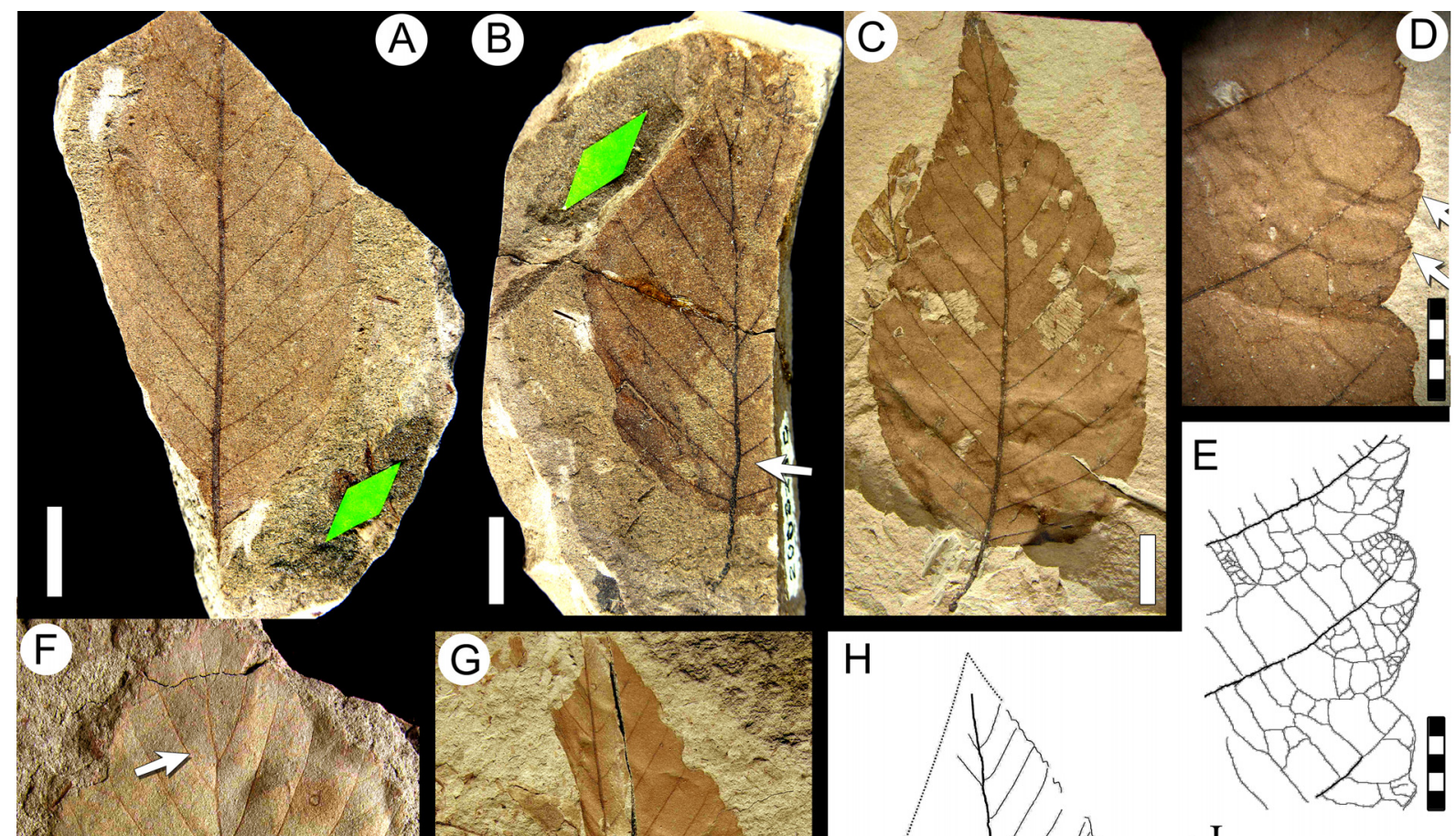

$\mathrm{E}$
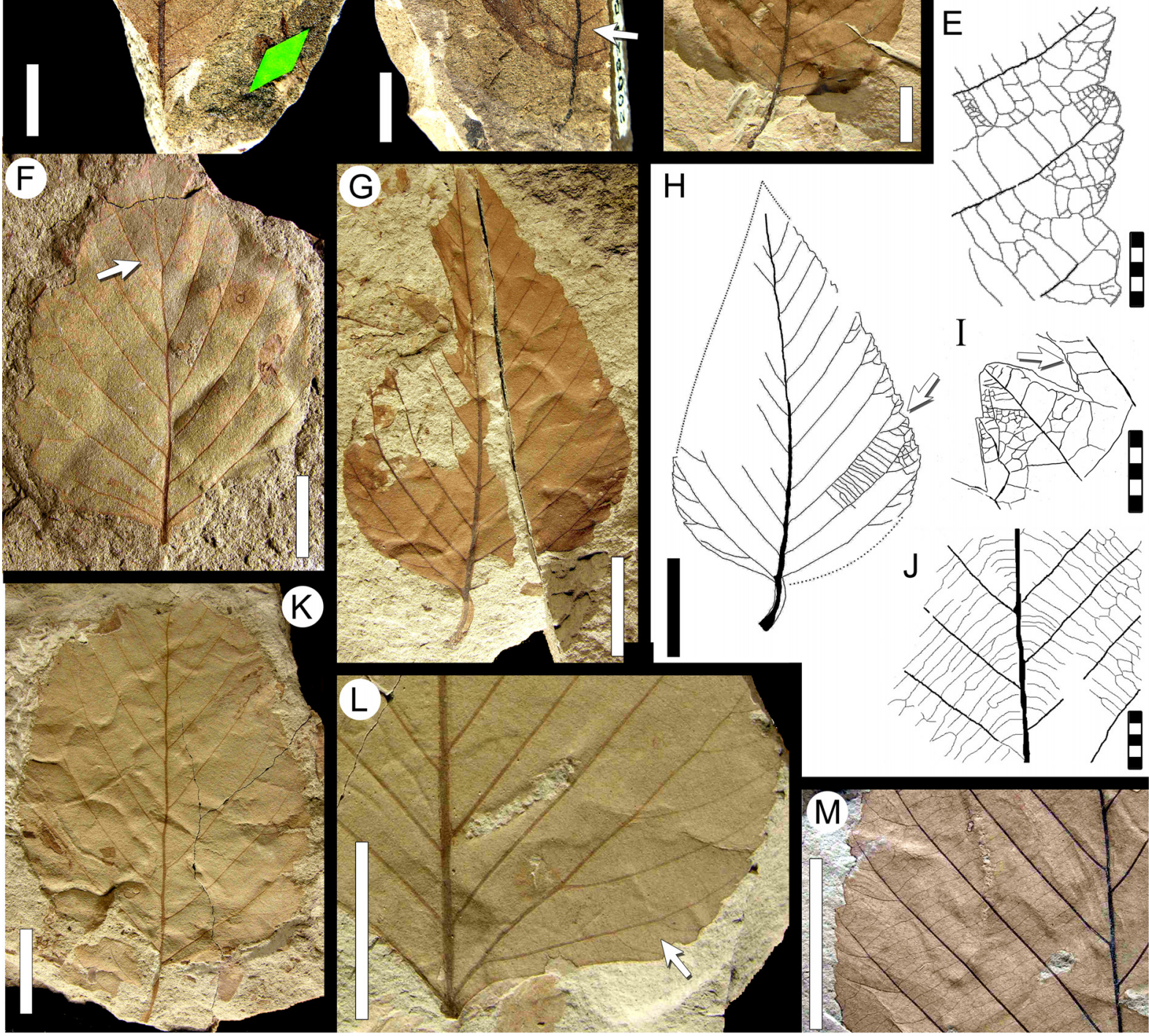

FIGURE 7. Morphotype SA004 "Dryophyllum" australis Berry 1937a (Nothofagaceae) and related material. A, " $D$. australis" syntype specimen USNM-208522 (illustrated in Berry 1937a, plate VI figure 4; specimen referred in this work to morphotype SA042 Cunoniaceae, see also Tables 1, 2). B, "D." australis syntype specimen USNM-208523a (illustrated in Berry 1937a, plate VI, figure 5; see also Tables 1, 2) (arrow, primary vein sinuosity); C, MPEF-Pb-2022 (exemplar); D, MPEF-Pb-2053 (accessory exemplar; arrows, two basal branches from a secondary vein innervating teeth); E, camera lucida drawing (CLD) of tooth venation of specimen in Figure 7D (accessory exemplar); F, MPEF$\mathrm{Pb}-9007$ (arrow, primary vein sinuosity); G, MPEF-Pb-9008; $\mathrm{H}$, digital overlay drawing (DOD) of specimen in Figure 7G (arrow, agrophic veins); I, CLD of tooth venation in MPEF-Pb-9009 (arrow, accessory vein on basal flank); J, DOD of MPEF-Pb-9010; K, MPEF-Pb-9011; L, MPEF-Pb-9012 (arrow, basal branches of agrophic veins); M, MPEF-Pb4018. Single-color scale bars equal $10 \mathrm{~mm}$; grid scales equal one millimeter (per rectangle). 
apical flank. Sinuses angular (Figure 7E). Tooth spacing uniform, at approximately four per centimeter (Figure 7D). Tooth venation with a medial principal vein, course straight or slightly curved; medial vein emits branches to the minor teeth and generates loops toward tooth margins; one basal accessory vein often arises from the subjacent sinus (Figure 7E, 7I).

Observations. Morphotype SA004 is present through all three Paleocene time intervals in this study (Table 1). It is referrable to one (USNM208523, Figure 7B) of the two type specimens of "Dryophyllum" australis Berry, 1937 from Palacio de los Loros (Berry, 1937a; Iglesias et al., 2007). The numerous (192; Table 1) new specimens reported here are more completely preserved than Berry's fragmentary types, showing higher order venation and better details of the margin and teeth. The other syntype (USNM-208522, Figure 7A) differs from USNM-208523 and the new specimens presented here. USNM-208522 is equivalent to Fagophyllum dusenii Berry, 1937a (morphotype SA042 here), leaving USNM-208523 as the sole type specimen that matches Berry's written description of "Dryophyllum" australis.

Jones et al. (1988) re-described type specimens of Dryophyllum Debey ex Saporta, 1862 from the Paleocene of France, emended the generic diagnosis, and concluded that the type material has juglandaceous affinities. However, the emended diagnosis of Jones et al. (1988) does not match the characters observed in " $D$." australis (and our morphotype SA004), and thus we use quotations for the generic name here until the present taxon is formally revised. Morphotype SA004 has leaf architecture that better matches Nothofagaceae Kuprianova, 1962 and Betulaceae Gray, 1822 nom. cons., such as ovate leaf shape, compound teeth with medial principal veins, stout primary veins that are deflected at secondary junctions, agrophic veins, and strongly percurrent tertiaries. The accessory vein of the teeth arises from the sinus, as in extant Nothofagus alessandri Espinosa, 1926 of South America.

Morphotype SA004 is similar to many fossils assigned to Nothofagus Blume, 1851 nom. cons. from the Cretaceous and Paleogene of Chile, Argentina, and Antarctica, including N. magellanica (Engelhardt) Dusén, 1908; N. elongata Dusén, 1908; N. variabilis Dusén, 1908; and N. subferruginea (Dusén) Tanai, 1986 (Engelhardt, 1891; Dusén 1907, Romero and Dibbern 1985, Tanai 1986). Among these fossil species, the largeleaved N. zastawniakiae Dutra (in Dutra and Bat- ten, 2000) from Antarctica has the closest morphology to morphotype SA004.

\section{Morphotype SA005}

Affinity. Cunoniaceae? Brown, 1814 nom. cons. Exemplar. MPEF-PB-2023 from quarry PL2 (Figure $8 C-E)$.

\section{Figure 8}

Description. Blade medially symmetrical, base slightly asymmetrical; size microphyll to notophyll, generally microphyll, mean length $6.3(2.5-8) \mathrm{cm}$, mean width $2.0(0.5-2.7) \mathrm{cm}$. Length:width ratio $4: 1$ (3.7-4.2). Margin dentate. Petiole insertion marginal, angled to the main leaf axis (Figure 8B, 8L). Shape ovate-lanceolate (Figure 8B, 8D, 8F). Apex shape straight; angle narrow-acute (Figure 8B, 8D, $8 \mathrm{~F}$ ). Base shape decurrent, angle acute (Figure $8 \mathrm{~B}, 8 \mathrm{D}, 8 \mathrm{~L}$ ). Primary venation pinnate, primary vein stout, course slightly curved. Basal veins three, the medial vein and one pair of acute basal secondaries (Figure 8D). Agrophic veins absent. Secondary venation semicraspedodromous to weakly brochidodromous toward the apex, loops thin close to blade margin, with 13-15 moderately curved vein pairs, subopposite to midvein, spacing increasing towards base (Figure 8D, 8F). Secondaries at acute angles to midvein, smoothly decreasing towards the base. Secondaries decurrent on midvein. Perimarginal venation a fimbrial vein welldeveloped at base (Figure 8B, 8D), tapering apically. Intersecondary veins thin, proximally parallel to major secondaries, course irregular (Figure 8D, $8 \mathrm{~F}$ ), length less than half of subjacent secondary, frequency one or more per secondary. Intercostal tertiary venation random reticulate to opposite percurrent (Figure 8C, 8F, 8L). Epimedial tertiary venation mixed opposite-alternate percurrent, course straight or convex with high divergence angle from midvein, angle increasing basally (Figure $8 \mathrm{~F}$ ). Fourth and fifth order veins regular polygonal reticulate (Figure $8 \mathrm{H}$ ). Areolation moderately developed, shape pentagonal. Marginal ultimate venation looped (Figure 8H). Teeth small, not present at base of blade, very reduced in smaller leaves (Figure 6B). Apex always with a darkened gland (Figure 8G-H, 8J). Tooth shape convex/convex or straight/convex, sinuses angular. Tooth spacing uniform, three to five per centimeter (Figure $8 \mathrm{~J}, 8 \mathrm{M}$ ). Tooth venation supplied by a medial principal vein arising from a secondary branch that departs from secondary vein loops near the tooth base; accessory venation looped (Figure 8G, 8H, $8 \mathrm{~K})$. 


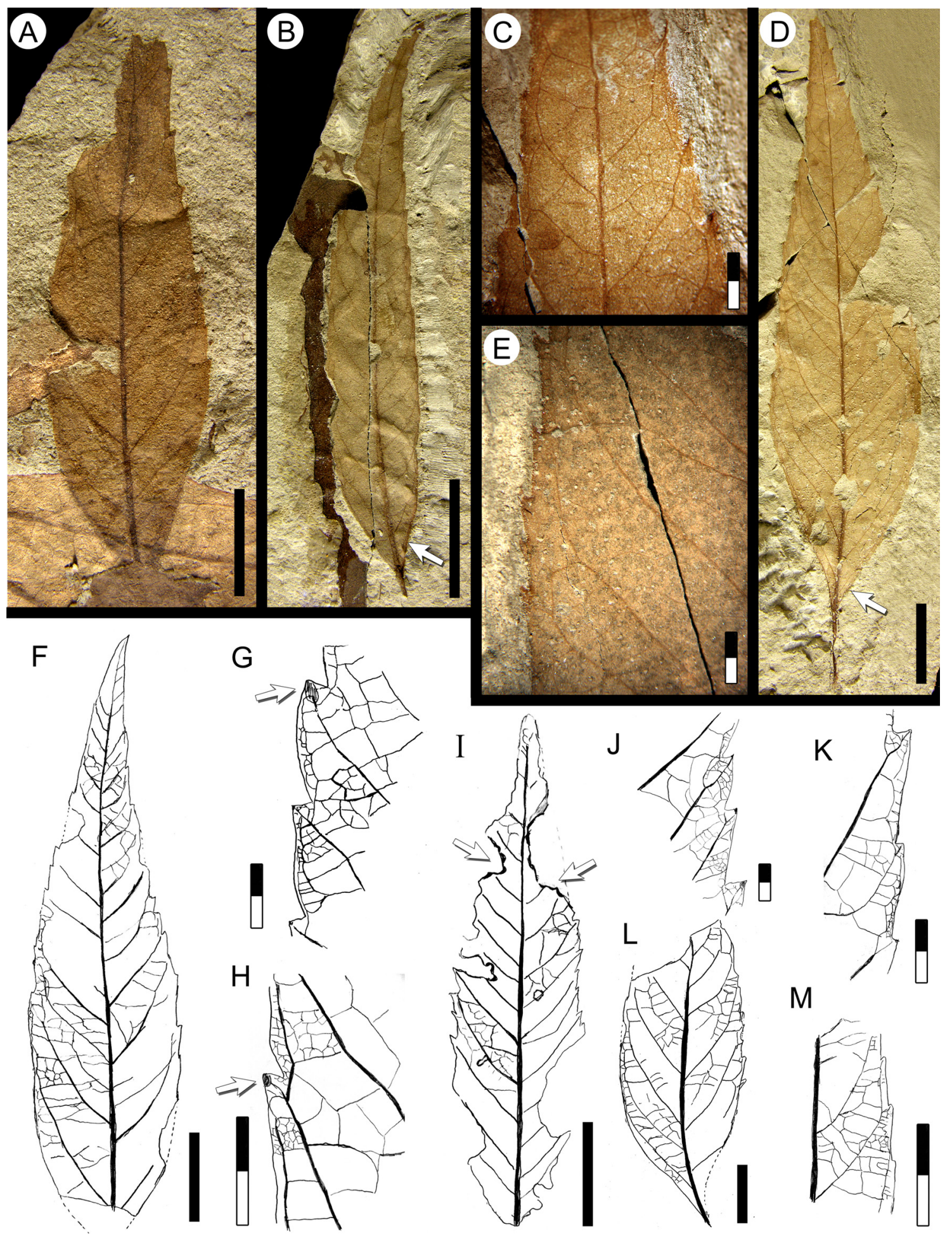

FIGURE 8. Morphotype SA005 (Cunoniaceae?). A, MPEF-Pb-4021; B, MPEF-Pb-4023 (arrow, basal portion of fimbrial vein); C, apical tooth detail of specimen in Figure 8D; D, MPEF-Pb-2023 (exemplar; arrow, basal portion of fimbrial vein); $\mathrm{E}$, medial tooth detail of specimen in Figure 8D; F, camera lucida drawing (CLD) of leaf venation of specimen in MPEF-Pb-9017; G, CLD of MPEF-Pb-9013 (arrow, darkened apical gland); H, CLD of tooth venation of MPEF-Pb-9014 (arrow, darkened apical gland); I, CLD of leaf venation of MPEF-PB-9015, note margin-feeding insect damage (DT12, arrows); J, CLD of tooth venation of specimen in Figure 8I; K, CLD of tooth venation of specimen in Figure 8G; L, CLD of leaf venation of MPEF-Pb-9016; M, tooth venation detail from leaf in Figure 8F. Single-color scale bars equal $10 \mathrm{~mm}$; grid scales equal one millimeter (per rectangle). 
Observations. Morphotype SA005 is present in all three time intervals studied here (Table 1). SA005 compares most closely with SA001 but differs in having a thick fimbrial vein along the full margin, more organized secondaries, and overall smaller tooth size. The asymmetrical base and blade of morphotype SA005 indicate a probable origin from a compound leaf, which along with the cunonioid teeth (Hickey and Wolfe, 1975) and minor order venation indicate a probable affinity with Cunoniaceae nom. cons. The presence of Cunoniaceae in the Paleogene of Patagonia is well established from Schizomerieae flowers from several of the same localities in the Salamanca and Peñas Coloradas formations (Jud et al., 2018a; see Introduction) and fruits assigned to Ceratopetalum Smith, 1793 from the early Eocene Laguna del Hunco site (Gandolfo and Hermsen, 2017).

\section{Morphotype SA008}

Affinity. Rosaceae Jussieu, 1789.

Exemplar. MPEF-PB-2024 from quarry PL1 (Figure 9A).

\section{Figure 9}

Description. Blade symmetrical; size nanophyll to mesophyll, generally microphyll; mean length 5.0 $(2.0-10.0) \mathrm{cm}$, mean width $3.0(0.8-5.9) \mathrm{cm}$, length:width ratio (1:1-2.6:1). Margin dentate. Petiole insertion marginal; petiole stout, base swollen, long, up to half of blade length (Figure 9l-J). Shape ovate. Apex shape straight or convex, angle acute (Figure 9A, 9C, 9F). Base shape concave, angle obtuse (Figure 9B-C). Primary venation pinnate. Primary vein moderate in thickness, course straight to leaf apex (Figure 9A-D). Basal veins one or three (when the first pair of basal secondaries arises basally, Figure 9C, 9E-F). Agrophic veins compound, irrigating basal teeth (Figure 9E, 9J). Secondary venation craspedodromous, veins in seven to nine opposite to subopposit vein pairs, spacing smoothly increasing toward base; angle acute and regular (Figure 9A-G, 9J). Secondaries bifurcate close to the margin: one branch irrigates a tooth medially, and the other branch reaches the supradjacent sinus (Figure 9B-C, 9K). Secondaries decurrent on midvein (Figure 9B-C, 9N). Perimarginal venation absent. Intersecondary veins generally absent, sometimes weakly expressed. Intercostal tertiary venation mixed opposite-alternate percurrent, becoming random reticulate toward margin (Figure $9 \mathrm{H}-\mathrm{K}$ ). Epimedial tertiary venation chevroned at base (Figure 9J), weakly opposite percurrent; course straight to convex with perpendicular departure from midvein. Departure angle increasing exmedially (Figure 9J-K). Fourth order veins regular polygonal reticulate. Marginal ultimate venation looped. Teeth compound, with up to three orders (Figure 9E-F). Tooth shape wide triangular; convex/convex, convex/straight, convex/ flexuous, or flexuous/flexuous; sinuses angular (Figure 9L). Tooth spacing uniform, one per centimeter. Tooth venation supplied by a medial principal vein corresponding to a branch of a secondary vein; lateral branches of the medial vein run to the supradjacent sinus with a curved course and fork, innerviating both sinus flanks; minor venation of the tooth is in several orders (Figure 9L-M).

Observations. Morphotype SA008 is present through all three Paleocene time intervals in this study (Table 1). The distinctive characteristics of morphoytpe SA008 are the craspedodromous secondaries that irrigate the teeth and send apical branches to the sinus or to minor teeth, the deeply incised compound teeth, and the compound agrophic veins, giving an overall morphology indistinguishable from living Rosaceae Jussieu, 1789 such as Crataegus Linnaeus, 1753 nom. cons. The leaves demonstrate a wide but continuous range of size and architecture, also similar to Crataegus. Larger leaves are markedly ovate, with dissected margins (Figure 9J). Fossils similar to SA008 have been described from the latest Cretaceous of Patagonia, including an identical morphotype from the Lefipán Formation (Stiles et al., 2020) and "Cissus" guidoensis Hünicken, 1995 from the Cerro Cazador Formation (Hünicken, 1995).

\section{Morphotype SA009}

Equivalent. "Cissites" patagonica Berry, 1937 in part: syntypes USNM-201955 (Berry, 1937a, plate VII, figure 1; here Figure 10N), USNM-201956 (Berry, 1937a, plate VII, figure 2; here Figure 10.OP), and USNM-201957 (Berry, 1937a, plate VII, figure 3; here Figure $10 \mathrm{Q}$ ) only (Table 2); syntype USNM-201959 excluded (see morphotype SA073). Affinities. Unknown.

Exemplar. MPEF-PB-2025 from quarry PL2 (Figure 10A).

Accessory exemplar. MPEF-PB-2054 from quarry PL2 (Figure 10J).

\section{Figures 10, 11}

Description. Blade symmetric or medially asymmetric, highly variable in size and shape; size nanophyll to macrophyll, generally mesophyll; mean length $7.7(2.0-16.0) \mathrm{cm}$, mean width 5.7 $(1.5-11.2) \mathrm{cm}$; length:width ratio (1:1-1.5:1). Margin finely serrate. Petiole base swollen, insertion marginal, length to $5 \mathrm{~cm}$, width to $2 \mathrm{~mm}$ (Figures $10 \mathrm{~A}$, 

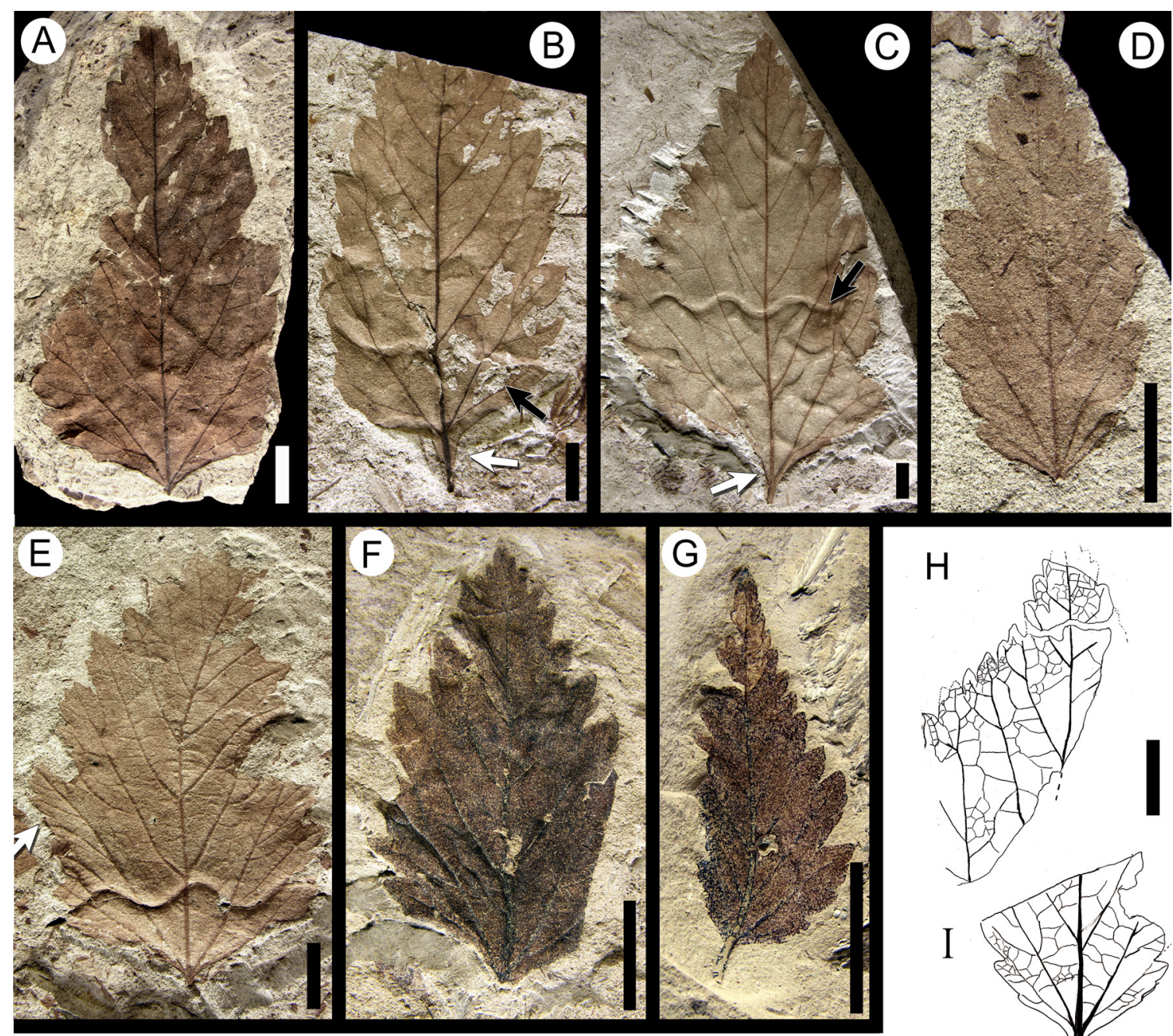

$\mathrm{H}$
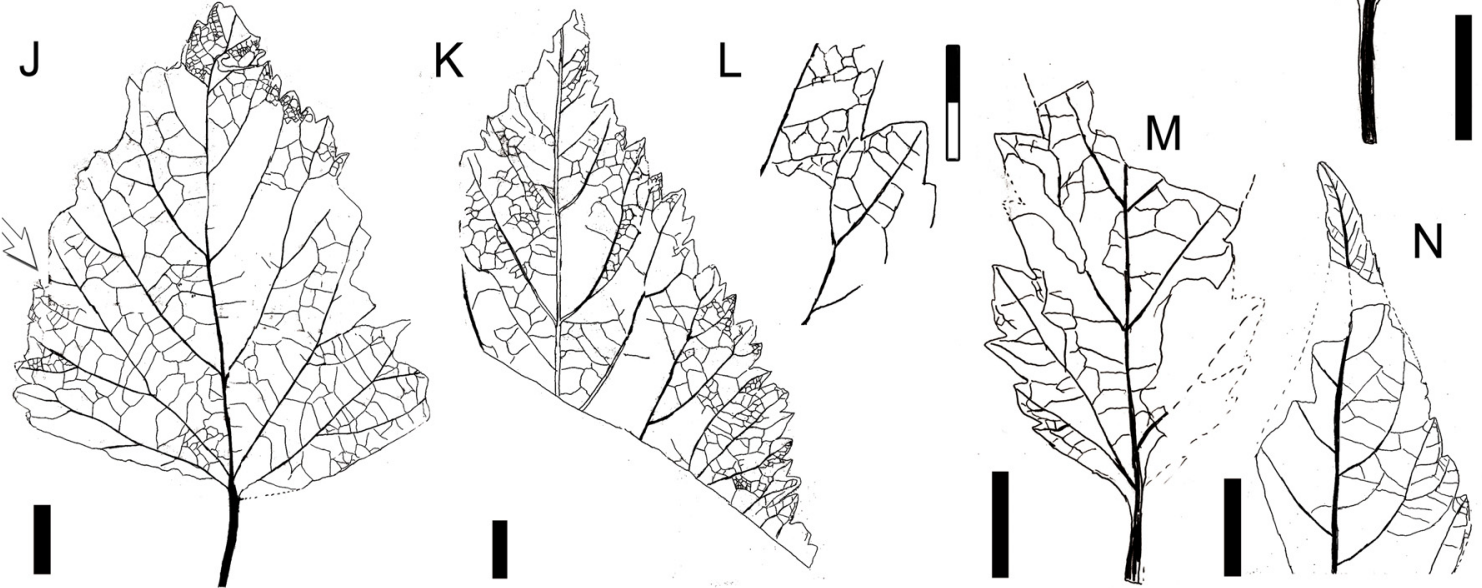

FIGURE 9. Morphotype SA008 (Rosaceae). A, MPEF-Pb-2024 (exemplar); B, MPEF-Pb-4028 (white arrow, concave base; black arrow, large polylobate hole feeding DT5, see also Donovan et al., 2018 figure 5.3); C, MPEF-Pb-4027 (white arrow, basal secondary veins), with Cochlichnus trace fossil (black arrow); D, MPרᄀEF-Pb-4030; E, MPEF-Pb4031 (arrow, compound agrophic veins), with Cochlichnus; F, MPEF-Pb-9018; G, MPEF-Pb-4033; H, camera lucida drawing (CLD) of leaf venation of MPEF-Pb-9019; I, CLD of leaf base of MPEF-Pb-9020; J, CLD of leaf venation of MPEF-Pb-9021 (arrow, compound agrophic veins); K, CLD of leaf venation of MPEF-PB-9022; L, CLD of tooth venation of MPEF-Pb-9023; M, CLD of leaf venation MPEF-Pb-9024; N, CLD of apical venation of MPEF-Pb-9025. Scale bars equal $10 \mathrm{~mm}$ except for 2 millimeter scale in Figure 9L. 


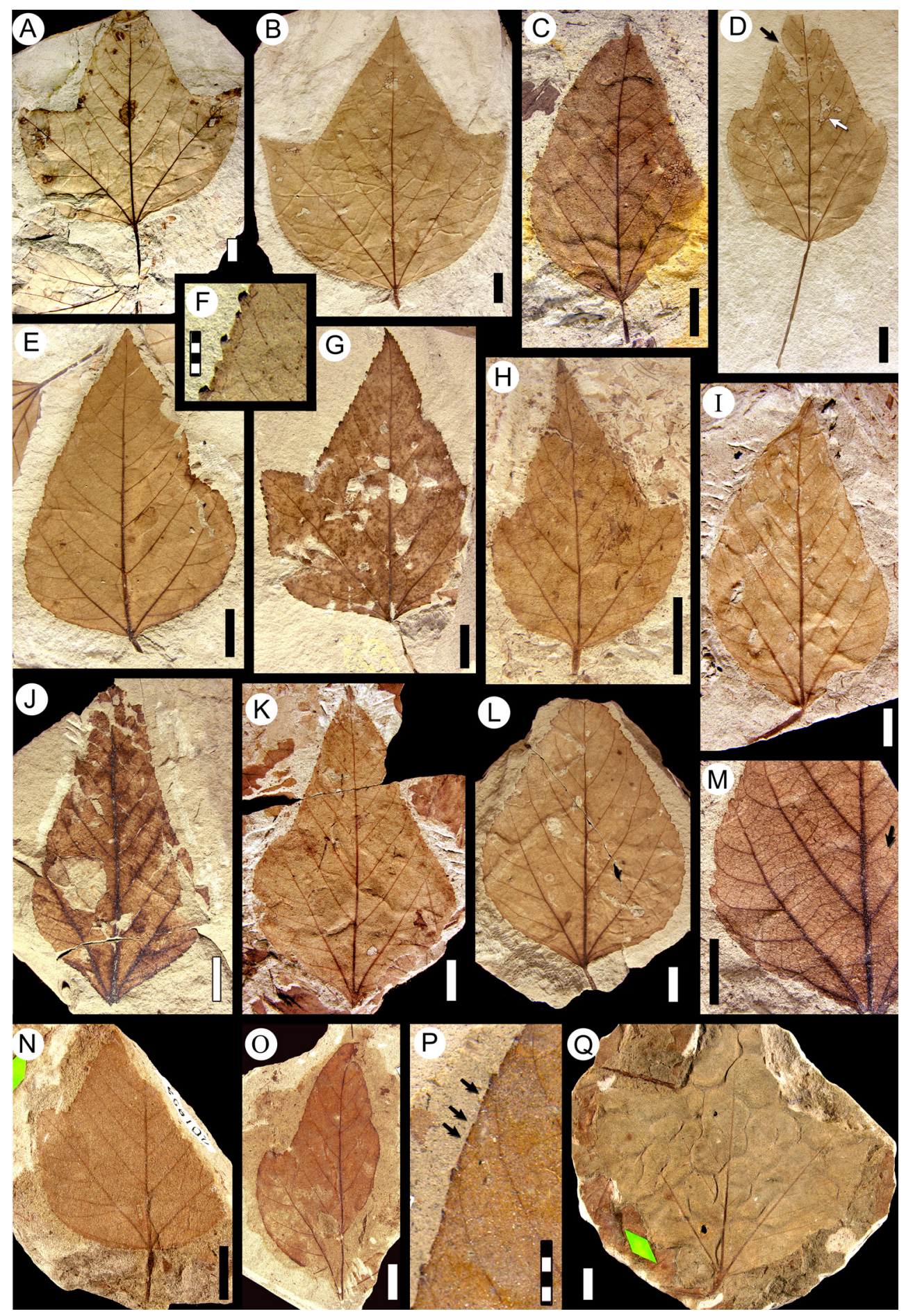

FIGURE 10. Morphotype SA009 “Cissites” patagonica Berry, 1937a (see also Figure 11). A, MPEF-Pb-2025 (exemplar); B, MPEF-PB-9026; C, MPEF-Pb-9027; D, MPEF-Pb-9028, note insect damage (black arrow, trenched margin feeding DT15; white arrow, hole feeding DT05; see also Donovan et al., 2018, figure 9.1); E, MPEF-Pb-4042; F, detail of glandular teeth of MPEF-Pb-9029; G, MPEF-Pb-4040; H, MPEF-Pb-4038; I, MPEF-Pb-4036; J, MPEF-Pb-2054 (accessory exemplar); K, MPEF-Pb-9031; L, MPEF-Pb-4041; M, detail of minor venation of MPEF-Pb-9032. N-Q, "C." patagonica syntypes; N, USNM-201955 (illustrated in Berry 1937a, plate VII, figure 1; see also Table 2); O, USNM201956a (illustrated in Berry 1937a, plate VII, figure 2; see also Table 2); P, tooth detail of specimen in Figure 100 (arrows, minute second-order teeth); Q, USNM-201957 (illustrated in Berry 1937a, plate VII, figure 3; see also Table 2), with Cochlichnus trace fossils. Scale bars equal $10 \mathrm{~mm}$, except millimeter scales (per rectangle) in Figure 10F and $10 \mathrm{P}$. 

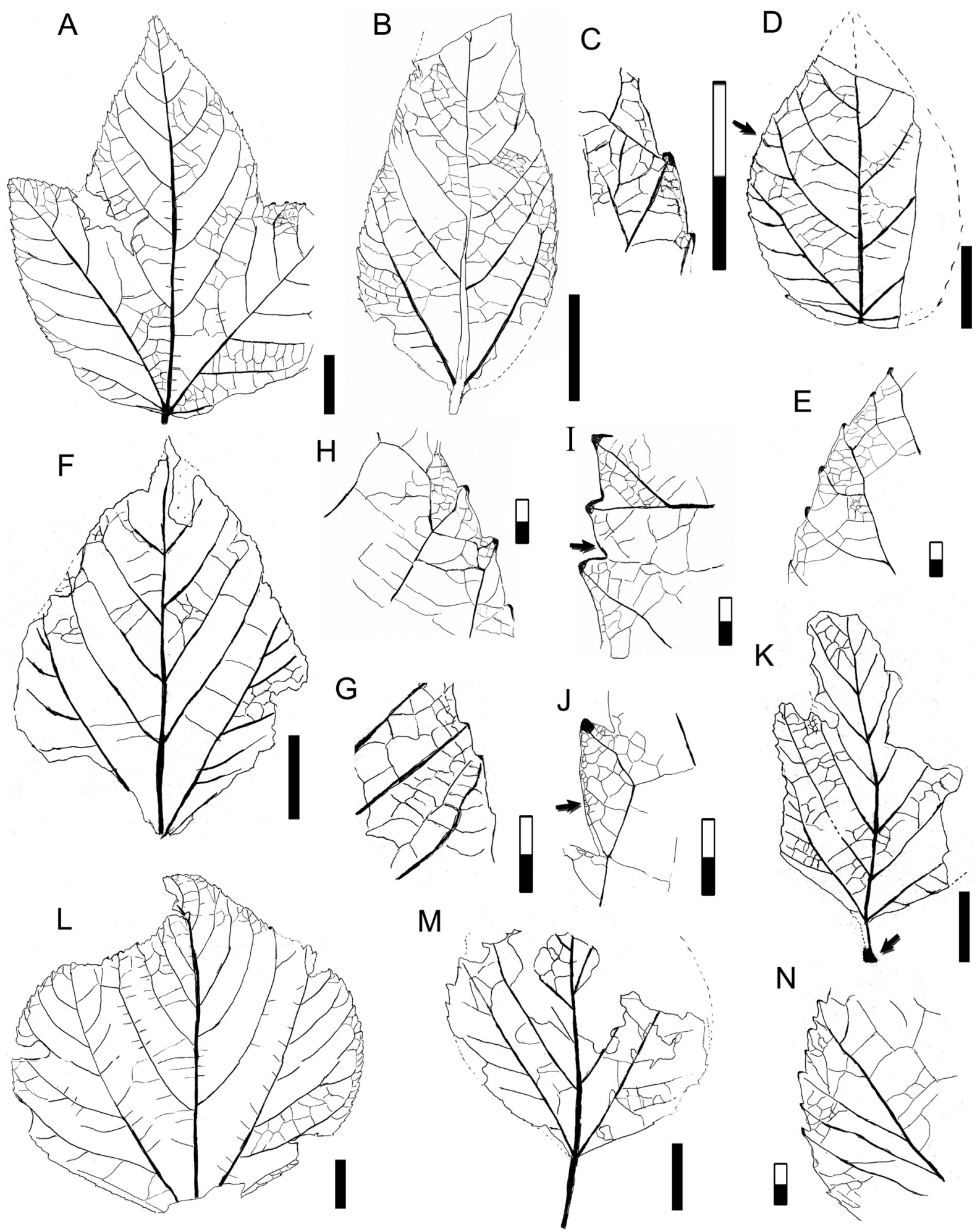

$\mathrm{N}$
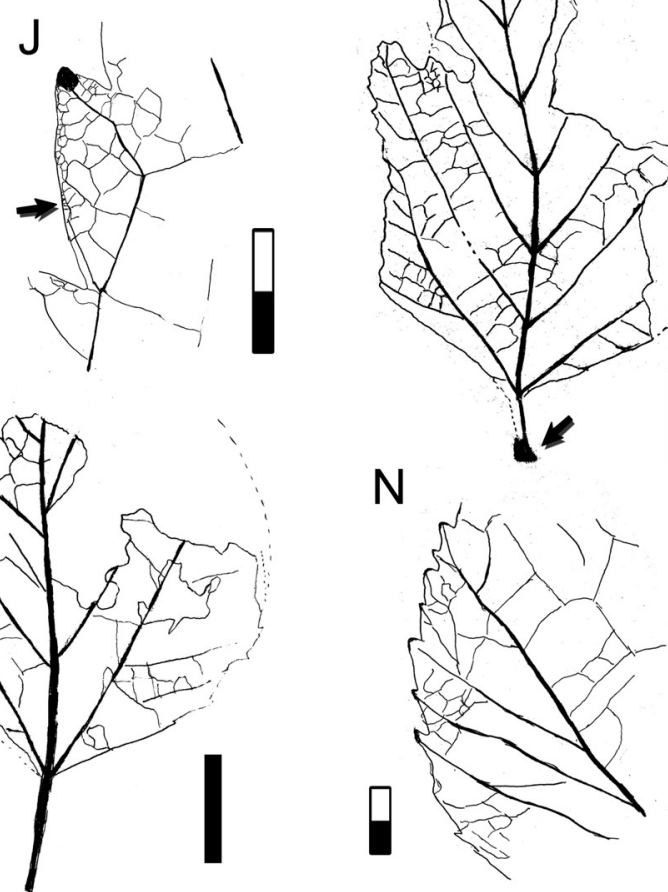

FIGURE 11. Morphotype SA009 "Cissites" patagonica Berry 1937a (continued from Figure 10), camera lucida drawings. A, MPEF-Pb-9020; B, MPEF-Pb-9152; C, tooth detail from Figure 11B; D, MPEF-Pb-9151 (arrow, compound agrophic veins); $\mathrm{E}$, tooth detail from Figure 11D; F, MPEF-Pb-9145; G, tooth detail from Figure 11F; $\mathrm{H}$, tooth detail from Figure 11A; I, tooth detail from Figure 11A (arrow, thin fimbrial vein); J, tooth venation detail from Figure 11A (arrow, looped marginal ultimate venation); K, MPEF-Pb-9160 (arrow, swollen petiole base); L, MPEF-Pb-9148; M, MPEF-Pb9146; N, tooth detail from Figure 11B. Scale bars equal $10 \mathrm{~mm}$ (per rectangle). 
10D, 10I, 11K, 11M). Shape varies from ovate unlobed to palmately 3-lobed. Apex shape convex to straight, occasionally concave if not well extended; angle acute. Base shape convex in narrow leaves (Figure 10J-M), rounded in wide leaves (Figure 10A, 10C-D, 10N); angle obtuse. Primary venation pinnate, midvein course straight. Basal veins five (Figure 10A-I). Agrophic veins may be simple in lobed leaves (Figures 10A-B, 10G-H, $11 \mathrm{~A}$ ) or compound in unlobed leaves (Figures 10E, 10J-M, 11B, 11D, 11L), and developed as semicraspedodromous. Secondary venation craspedodromous, with five to nine pairs of opposite to subopposite excurrent veins, the two basal pairs slightly thicker than other veins (Figure 10A-I). Secondary course slightly curved, unbranched; vein spacing uniform, angle acute and smoothly decreasing toward base in unlobed forms (Figure $10 \mathrm{C}$ ), and more uniform in lobed forms (Figures $10 \mathrm{~A}, 11 \mathrm{~A})$. Perimarginal venation a very thin fimbrial vein, thickening the leaf margin slightly (Figure $10 \mathrm{P}, 11 \mathrm{C}, 11 \mathrm{I})$. Intersecondary veins very thin or absent (Figures 10M, 11D). Interior secondaries present. Intercostal tertiary venation mixed opposite-alternate percurrent, sometimes random reticulate, arising at perpendicular angles to secondaries (Figure 11A-D, 11F-G, 11K). Epimedial tertiary venation mixed opposite-alternate percurrent, obtuse to primary, angle uniform (Figure 11A). Fourth order veins regular polygonal reticulate (Figure 11A, 11K). Areoles four-five sided, width to $0.9 \mathrm{~mm}$. Marginal ultimate venation looped (Figure 11E, 11G-J). Teeth small, asymmetrical, simple or compound with two orders (Figures 10P, 11G-H). Tooth shape convex/convex, convex/flexuous, straight/flexuous, or flexuous/flexuous; the apical flanks shorter than the basal flanks. First order teeth arise at secondary vein terminations; the additional tooth orders arise between the primary teeth (Figures 10F, 10P, 11E). Tooth apex glandular or with a small spherulate callosity fused to the apex (Figures 10F, 11E, 11G-J). Sinuses rounded. Tooth spacing uniform, about six per centimeter (Figures 10P, 11E). Tooth venation supplied by a thick, straight or deflected medial principal vein; accessory veins looped (Figure 11I-J).

Observations. Distinguishing characters of this morphotype include: blade trilobate or ovate; primary vein pinnate; secondary veins craspedodromous, not ramified; agrophic veins present; petiole long; and teeth numerous and small (salicoid), in two orders with glandular apices (see key in supplementary Appendix 2). Morphotype SA009 is referrable to "Cissites" patagonica Berry, 1937, originally described from Palacio de los Loros (Cerro Funes). However, among Berry's (1937a) four syntypes (Table 2), specimen USNM-201959 (Figure 36C) does not show the same characters listed in Berry's description and as seen in the other syntypes. Instead, USNM-201959 has an untoothed margin and correlates to our morphotype SA073 (Table 2) below. Morphotype SA009 is present through all three Danian time intervals considered here and is usually very abundant (Table 1). Berry's (1937a) original assignment to Vitaceae Jussieu, 1789 nom. cons. is dubious because lobed forms in that family characteristically have cordate or lobate bases.

\section{Morphotype SA010}

Equivalent. Laurophyllum piatnitzkyi Berry, 1937 (syntypes USNM-201965, USNM-201966; Berry, 1937a, plate IX, figures 1-2; here Figure 12A); and Cryptocaryoides maria-santisimensis Berry, 1937 (syntypes USNM-208520, USNM-208521; Berry, 1937a, plate VI, figure 3; here Figure 12B) (Table 2).

Affinity. Lauraceae Jussieu, 1789.

Exemplar. MPEF-PB-2026 from quarry PL2 (Figure 12M).

Accessory exemplar. MPEF-PB-2055 from quarry PL2 (Figure 12H-I).

Figures 12-13

Description. Blade simple, symmetrical; size nanophyll to macrophyll, generally notophyll. Mean length 8.1 (2.5-14.0) $\mathrm{cm}$, mean width 3.7 (1.3-8.4) $\mathrm{cm}$; length:width ratio variable (0.7-1:1-2-3:1). Margin entire, sometimes wavy (Figure 12E, 12K-L). Petiole thick, insertion marginal, length to $1 \mathrm{~cm}$, width to $3 \mathrm{~mm}$ (Figures 12F, 12L, 13D, 13G). Shape elliptic, tending to ovate (Figures 12E, 12M, $13 \mathrm{E}, 13 \mathrm{H}$ ) or narrow elliptic (Figures 12F-G, 13A$B)$. Apex shape acuminate, variably extended to form a drip tip (Figures 12N, 13A, 13C, 13G), angle acute. Base shape decurrent, angle generally acute but may be obtuse (Figure 13H). Primary venation pinnate with a thick primary vein deflected by secondaries (Figures 12M, 13A). Basal veins three, one primary and two fimbrial veins (Figure 12M). Agrophic veins variably present, usually absent in smaller leaves, simple (Figures 12F, 13G-H), sometimes compound (Figures 12E, 12K, $12 \mathrm{M}, 13 \mathrm{~F}$ ) with uniformly curved branches forming loops. Secondary venation brochidodromous, with opposite to alternate disposition, joining the supradjacent secondary by a series of loops parallel to the margin (Figures 12E, 13C, 13H). Vein course straight or curved, markedly thinner towards apex. 


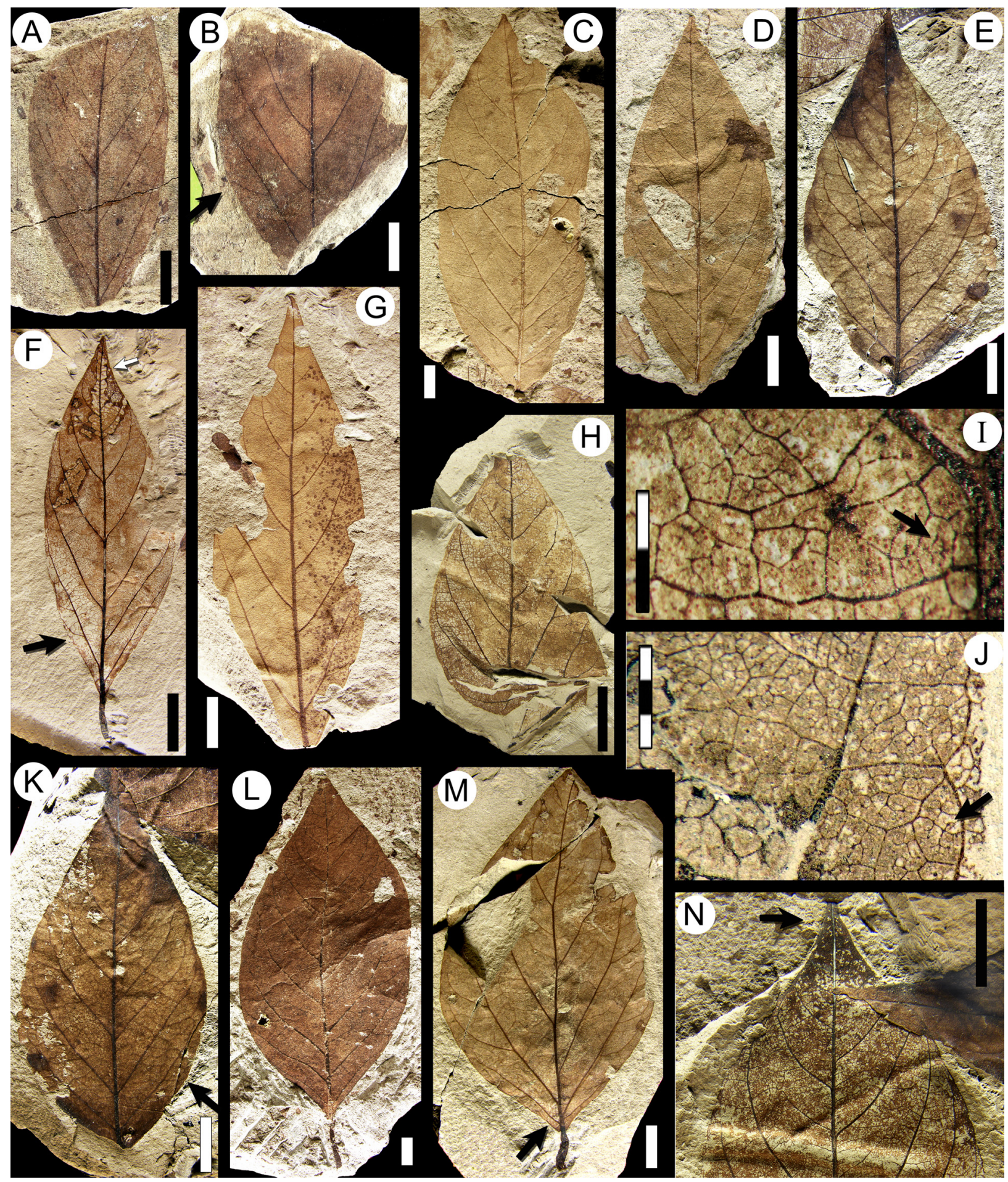

FIGURE 12. Morphotype SA010 (Lauraceae, see also Figure 13). A, Laurophyllum piatnitzkyi Berry, 1937a syntype specimen USNM-201966 (illustrated in Berry, 1937a, plate IX, figure 2; see also Tables 1, 2); B, Cryptocaryoides maria-santisimensis Berry, 1937a syntype specimen USNM-208521 (illustrated in Berry, 1937a, plate VI, figure 3; see also Tables 1,2) (arrow, agrophic veins); C, MPEF-PB-9033; D, MPEF-PB-9034; E, MPEF-Pb-4048; F, MPEF-Pb-9035 (black arrow, agrophic veins), with skeletonization (DT79, white arrow; see also Donovan et al., 2018, figure 9.23); G, MPEF-Pb-3036; H-I, MPEF-Pb-2055 (accessory exemplar), note idioblasts (dark dots, arrow) preservation; J, detail of areoles and FEVs associated with idioblasts (arrow) from Figure 12F; K, counterpart of specimen in Figure 12E (arrow, agrophic veins); L, MPEF-Pb-9039; M, MPEF-Pb-2026 (exemplar; arrow, basal portion of fimbrial vein); N, MPEF-Pb-4049 (arrow, drip tip). Single-color scale bars equal $10 \mathrm{~mm}$; grid scales equal one millimeter (per rectangle). 

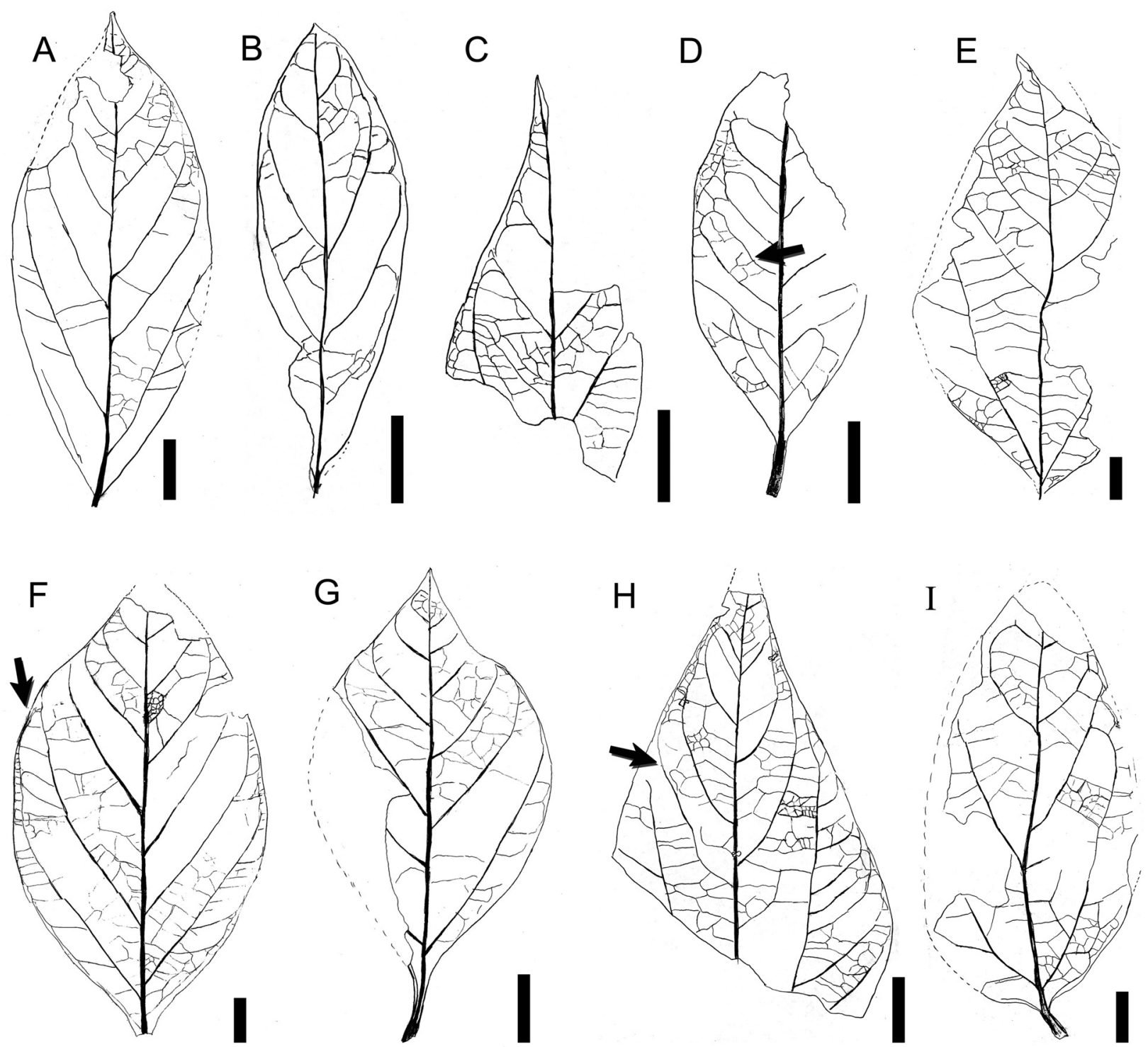

FIGURE 13. Morphotype SA010, Lauraceae (continue from Figure 12), camera lucida drawings. A, MPEF-Pb-9042; B, MPEF-Pb-9044; C, MPEF-PB-9049; D, MPEF-Pb-9051 (arrow, simple intersecondary vein); E, MPEF-Pb-9052; F, MPEF-Pb-9045 (arrow, compound agrophic veins); G, MPEF-Pb-9046; H, MPEF-Pb-9047 (arrow, simple intersecondary vein); I, MPEF-Pb-9048. Scale bars equal $10 \mathrm{~mm}$.

Spacing smoothly increasing toward base (Figures 12G, 13G); angle may decrease abruptly in the basal pair (Figure 13E) or remain uniform toward base. In some specimens (e.g., Figures 12G, 13C, $13 \mathrm{E}, 13 \mathrm{H})$, the angle of the basal secondaries is especially acute, and secondary veins may extend along over a third of the blade length. Secondaries decurrent on midvein (Figures 12F, 12M, 13A-B). Perimarginal venation a pronounced fimbrial vein, running along the full margin (Figure $12 \mathrm{M}$ ). Intersecondary veins simple (Figure 13D, 13H) or composite (Figure 13D), proximally parallel to major secondaries, length less than half of subjacent sec- ondary, frequency less than one per secondary. Intercostal tertiary venation opposite percurrent, perpendicular to secondaries (Figure 13E, 13I). Epimedial tertiary venation commonly opposite percurrent, may be mixed opposite-alternate percurrent, course straight, generally perpendicular to midvein (Figure 13E). Fourth order venation regular polygonal (Figures $12 \mathrm{~J}, 13 \mathrm{~F}$ ) to random reticulate (Figures 12I, 13H). Fifth order venation regular polygonal reticulate (Figure 12J, 12I). Areolation well developed, areoles four-five sided, width 0.3$1.0 \mathrm{~mm}$. Freely ending veinlets arise orthogonally, two or more branched, ending in small idioblasts 
(preserved as dark dots; Figure 12J). Vein density range $10.98-13.10 \mathrm{~mm} / \mathrm{mm}^{2}$. Marginal ultimate venation looped (Figure 13C, 13F, 13H).

Observations. Morphotype SA010 differs from other entire-margined, pinnate-veined leaf morphotypes studied here (i.e., morphotypes SA046, SA056, SA063, SA073, SA077, and SA078) in that it has a symmetrical blade, thick petiole, a drip tip, three basal veins including basal secondaries that may extend over a third of the blade length, intersecondary veins present, pronounced fimbrial vein, and presence of large and abundant idioblasts (see key in supplementary Appendix 2). Morphotype SA010 matches in architecture two species described from the historic Cerro Funes locality (Berry, 1937a): Laurophyllum piatnitzkyi Berry, 1937 and Cryptocaryoides maria-santisimensis Berry, 1937. Berry's type specimens are fragmentary (Figure 12A and 12B, respectively), but the new collection presented here has abundant specimens that are more completely preserved, including several with Lauraceae-type cuticle under separate study. The L. piatnitzkyi syntypes have several characters that match the more elliptical examples of morphotype SA010, whereas the $C$. maria-santisimensis syntypes correspond to our larger, wider, tending-to-ovate specimens. The new collection presented here shows these morphologies as end members along with the full range of intermediates (Figures 12,13). This morphotype is present at each sampling level and is often the most common leaf form (Table 1). The significant variation suggests that it could represent several biological species, a hypothesis that could be tested using the preserved cuticle.

\section{Morphotype SA014}

Equivalent. "Sterculia" acuminataloba Berry, 1937 in part: syntypes USNM-208524 (Berry, 1937a, plate VIII, figure 1; here Figure 14A), USNM208526 (Berry, 1937a, plate VIII, figure 3; here Figure 14B), USNM-208527 (Berry, 1937a, plate VIII, figure 4) only (Table 2); syntypes USNM-208525 and USNM-208528 excluded (see morphotypes SA058 and SA019).

Affinity. Unknown.

Exemplar. MPEF-PB-2027 from quarry PL2 (Figure 14C).

Figures 14, 15A-D

Description. Blade symmetrical; size nanophyll to microphyll, generally microphyll. Mean length 6.7 $(3-10.5) \mathrm{cm}$, mean width $7.6(1.3-13) \mathrm{cm}$, length:width ratio $\sim 1: 1$. Margin entire. Petiole insertion marginal, long (Figure 14E). Shape ovate to elliptic, palmately 3-5 lobed; smaller leaves may be 3-lobed, most leaves 5-lobed. Lobes large, usually deeply incised (Figure 14C,14I, 14N), up to $6.5 \mathrm{~cm}$ in length. Lobe length:width ratio 3.5:1. Lobe apex straight (Figure 14C), rarely convex (Figure 14DE). Base shape decurrent (Figure 14E, 14N, 15B), angle obtuse, may reach $180^{\circ}$ (Figure 15C-D). Primary venation actinodromous (Figures 14E, 15B) to rarely palinactinodromous (Figure 14B-C, 14L), origin variably basal or suprabasal (Figure $14 \mathrm{H}$, 14K-L). Primary vein course regular, slightly curved, unbranched except by palinactinodromy. Basal veins five primaries. Agrophic veins absent. Secondary venation either terminating in intramarginal vein and joining it at right angles (Figures $14 \mathrm{M}, 15 \mathrm{~B}$ ), weakly brochidodromous close to margin (Figures 14A, 14E-F, 14K, 15A), or interior near the base (Figure 15A). Sinus-bracing veins arise from both medial and lateral primaries, reaching the margin perpendicularly, forking near the sinus, and joining the intramarginal vein (Figures 14F, 15A-B). Spacing irregular, usually one or two secondaries per centimeter. Angle irregular, opposite to alternate. Secondaries decurrent on midvein (Figures 14E-F, 15B). Perimarginal venation a welldeveloped intramarginal vein, slightly irregular, may be thickened near the base as a fimbrial vein (Figures 14E-F, 15B). Intersecondary veins thin, proximally parallel to subjacent secondary, less than half the length of the subjacent secondary, distally perpendicular to subjacent secondary, one or two per secondary (Figures 14E, 15A-B, 15D). Intercostal tertiary venation random reticulate (Figure $14 \mathrm{~J}$ ). Epimedial tertiary venation random reticulate (Figure 15B). Fourth and fifth order venation regular polygonal reticulate (Figure 14J). Areolation well developed, areoles pentagonal, width less than $0.3 \mathrm{~mm}$. Freely ending veinlets absent. Vein density range $8.78-10.0 \mathrm{~mm} / \mathrm{mm}^{2}$. Marginal ultimate venation looped (Figures 14J, 15A-B).

Observations. Morphotype SA014 can be distinguished from other palmately lobed morphotypes (morphotypes SA019 and SA058) by a number of features including: blade palmately 3-5 lobed; lobes simple, large, with straight apex; primary veins actinodromous (rarely palinactinodromous), not branched; secondary veins thin, brochidodromous; and a conspicuous intramarginal vein (see key in supplementary Appendix 2). Morphotype SA014 corresponds to the type specimens of "Sterculia" acuminataloba Berry, 1937a from Palacio de los Loros indicated above. The excluded syntypes USNM-208525 and USNM-208528 (Figures 32I and $17 \mathrm{I}$, respectively) do not show the same mor- 


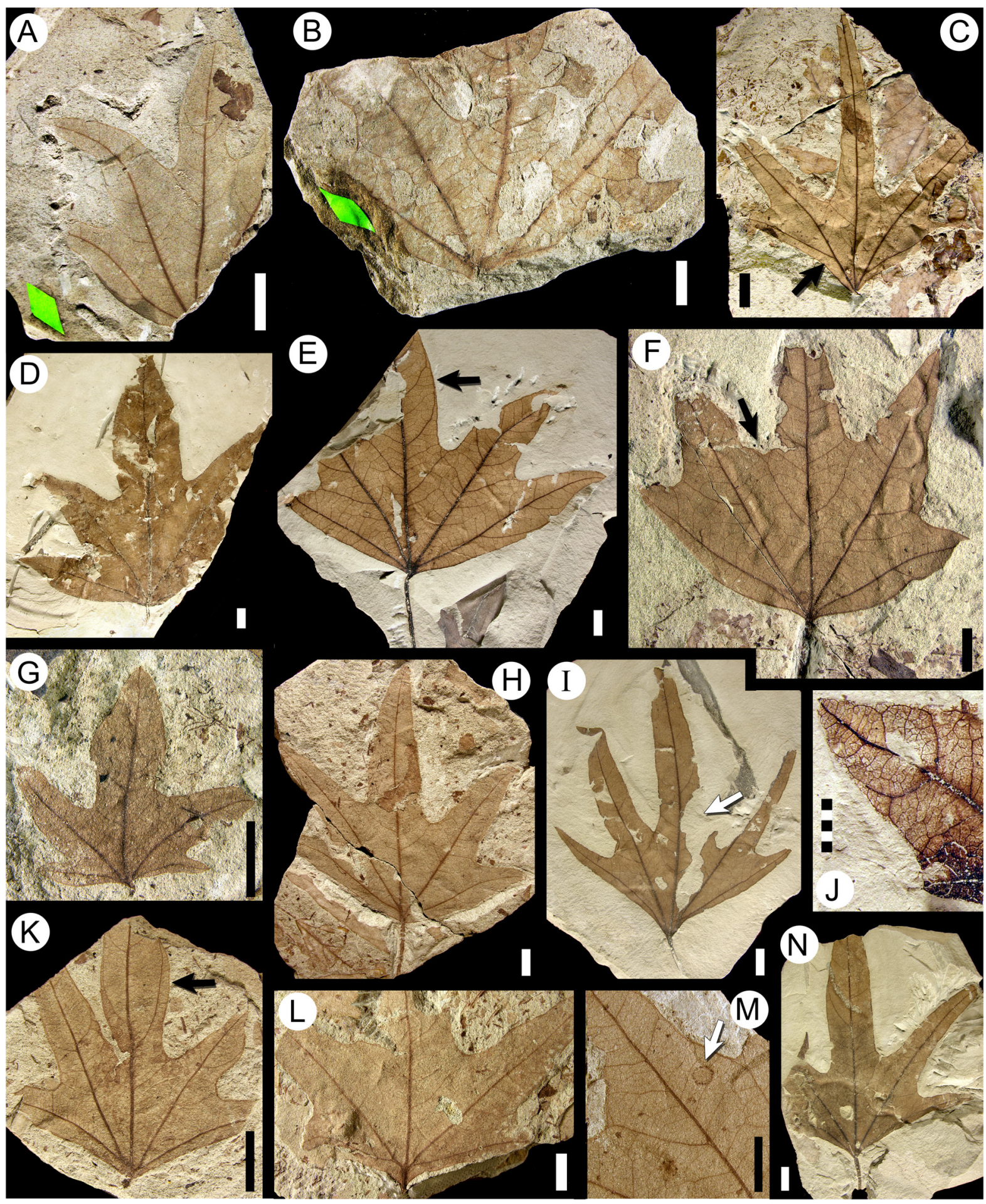

FIGURE 14. Morphotype SA014, "Sterculia" acuminataloba Berry 1937a (see also Figure 15). A, "S." acuminataloba syntype USNM-208524 (illustrated in Berry, 1937a, plate VIII, figure 1; see also Table 2); B, "S." acuminataloba syntype USNM-208526 (illustrated in Berry, 1937a, plate VIII, figure 3; see also Table 2); C, MPEF-Pb-2027 (exemplar; arrow, palinactinodromous venation); D, MPEF-Pb-4058; E, MPEF-Pb-9053 (arrow, weak brochidodromous secondary vein); F, MPEF-Pb-9054 (arrow, sinus-bracing vein); G, MPEF-Pb-4056; H, MPEF-Pb-4057; I, MPEF-Pb-4070, with margin feeding (DT14, arrow); J, lobe venation detail of MPEF-Pb-9056; K, MPEF-Pb-4053 (arrow, weak brochidodromous secondary vein); L, MPEF-Pb-9057; M, sinus-bracing veins, detail of MPEF-Pb-9058, and a fungal pycnidium or stroma leaf damage ring (arrow, DT334; see also Donovan et al., 2018 figure 6.13); N, MPEF-Pb-4059. Scale bars equal $10 \mathrm{~mm}$, except 5 millimeter scale in Figure $14 \mathrm{~J}$. 

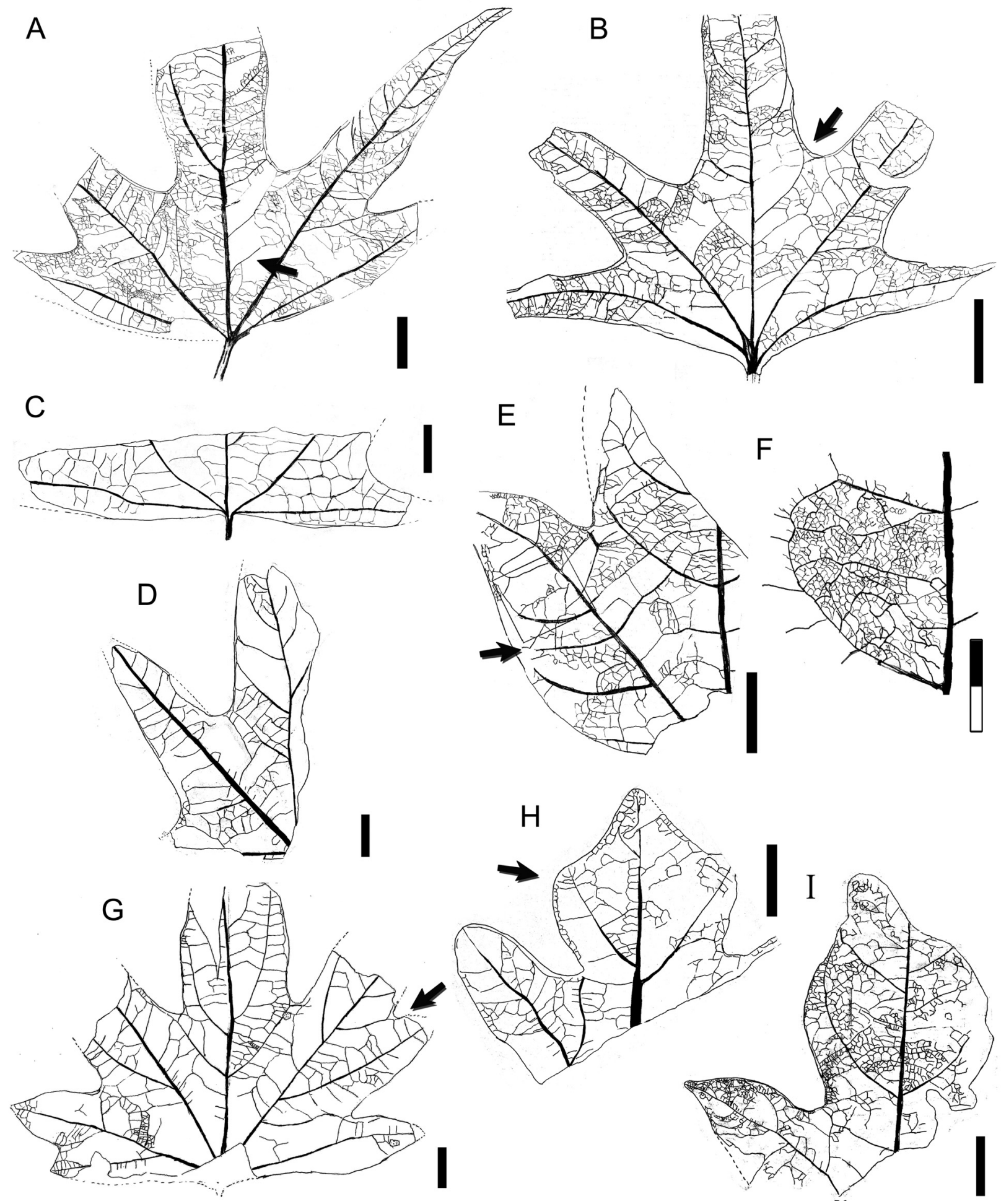

FIGURE 15. Morphotype SA014 "Sterculia" acuminataloba Berry 1937a (continued from Figure 14) and morphotype SA019 (see also Figure 17). A-D, camera lucida drawings (CLD) of morphotype SA014 "Sterculia" acuminataloba. A, MPEF-Pb-9059 (arrow, interior secondary vein); B, MPEF-Pb-9060 (arrow, sinus-bracing vein); C, MPEF-Pb-9061; D, MPEF-Pb-9062. E-I, CLD of morphotype SA019. E, MPEF-Pb-9063 (arrow, minor secondary vein); F, minor venation detail of specimen in Figure 15E; G, MPEF-Pb-9064 (arrow, sinus-bracing vein); H, MPEF-Pb-9065 (arrow, secondary lobe); I, MPEF-Pb-9066. Scale bars equal $10 \mathrm{~mm}$, except 2 millimeter scale in Figure $15 \mathrm{~F}$. 
phology as Berry's written description for the species and the other type material, instead corresponding to our morphotypes SA058 and SA019, respectively. Morphotype SA014 has been found at most localities discussed here and is abundant in most of them (Table 1). There are a large number of fossil leaf species from the Cretaceous and Paleogene of southern South America that are assigned to Sterculia Linnaeus 1753 (Berry, 1928; Berry, 1937b; Menéndez, 1959; Arguijo and Romero, 1981; Hünicken, 1995). None of them is clearly assignable to the living genus, nor do they appear to correspond to morphotype SA014, which is present through all three Paleocene paleomagnetic chrons sampled here (Table 1).

\section{Morphotype SA016}

Possible Affinity. Unknown.

Exemplar. MPEF-PB-2028 from quarry PL2 (Figure 16A-B).

Figures 16A-E, 19F-I

Description. Blade symmetric to slightly asymmetric; size nanophyll (Figures 16C-D, 19F) to microphyll, generally microphyll. Mean length 6.8 (310.5) $\mathrm{cm}$, mean width $1.7(0.4-2.3) \mathrm{cm}$, length:width ratio (4:1-7:1). Margin entire. Petiole stout, insertion marginal (Figure 16C-D). Shape narrow elliptic. Base and apex shape straight, angle acute. Primary venation pinnate, course smoothly curved and prominent to apex. Basal veins three, one primary and two secondary marginal veins that taper and disappear apically (Figure 19F). Agrophic veins absent. Secondary venation brochidodromous, with 9-11 pairs of opposite to alternate secondaries (Figures 16A, 16E, 19F-H). Course approximately straight, then reticulating (Figure 19G-H) and weakly looping toward margin (Figure 19F, 19I). Spacing and angles irregular; angles acute. Secondaries decurrent on midvein. Perimarginal venation an irregular intramarginal vein, formed from exterior secondary loops when consolidated (Figures 16B, 16D, 19F$\mathrm{G})$. Intersecondary veins thin, proximally parallel to subjacent secondary, more than half of the subjacent secondary length, distally reticulating or ramifying, one or two per secondary (Figures 16B, 19G$\mathrm{H})$. Intercostal tertiary venation random reticulate (Figures 16B, 19G). Epimedial tertiary venation reticulate. Fourth order veins thin, forming irregular polygons (Figure 16B). Fifth order veins freely ramifying. Areolation poorly developed. Freely ending veinlets dichotomously branching (Figure 16B). Vein density $11.64 \mathrm{~mm} / \mathrm{mm}^{2}$ (one leaf measured).
Areolation poorly developed. Marginal ultimate venation in two or more series or irregular loops (Figure 19F). Leaf rank 2r.

Observations. Distinguishing characters of morphotype SA016 include: blade narrow elliptic; primary veins pinnate; secondary veins densely spaced, weak brochidodromous or festooned brochidodromous; intramarginal vein irregular; and leaf rank $2 r$ (see key in supplementary Appendix 2). Morphotype SA016 is present through all Danian time intervals considered in this study (Table 1).

\section{Morphotype SA019}

Equivalent to. "Sterculia acuminataloba" Berry, 1937 in part: syntypes USNM-208528 (Berry, 1937a, plate VIII, figure 5; here Figure 17I) only (Table 2); syntypes USNM-208524-208527 excluded (see morphotypes SA014 and SA058).

Affinity. Unknown.

Exemplar. MPEF-PB-2029 from quarry PL2 (Figure 17A, 17D-E).

Figures 15E-I, 17

Description. Blade symmetrical or asymmetrical, size notophyll to macrophyll, generally mesophyll. Mean length 7.8 (3.5-19) cm, mean width 8.1 (2.620.0) $\mathrm{cm}$, length:width ratio (0.8-1.2:1). Margin technically entire but with reduced lobes resembling irregular teeth often present (Figure 17A, 17L). Petiole insertion marginal (Figure 17E, 17J), long (Figure 17G), width up to $1.9 \mathrm{~mm}$. Shape ovate, palmately odd lobed with up to six lobes (Figures 15G-H, 17L), the lobes compound, occasionally simple (Figure $17 \mathrm{H}$ ), lobe sinuses rounded (Figure 17C); lobe apex shape straight (Figure 17C) or convex (Figures 15I, 17G-H), angle obtuse. Base shape cordate (Figure 17A, 17F, $17 \mathrm{H}, 17 \mathrm{~L}$ ) or rounded (Figure 17J-K), angle obtuse, generally $>180^{\circ}$. Primary venation basally actinodromous (Figure 17G-L), rarely palinactinodromous (Figure 17A, 17F). Primary vein course straight (Figure $17 \mathrm{H}$ ) or slightly sinuous, midvein characteristically deflected by thick secondaries (Figure 15G, 17A-B, 17I). Basal veins seven (Figure $17 \mathrm{~K}$ ) to eight (Figure 17E, 17L), five or six basal primaries plus two lateral intramarginal veins (Figure 17E). Agrophic veins absent. Secondary venation irregular, variably craspedodromous (when entering lobes and lobules directly or through branching), eucamptodromous (usually in simple lobes, Figure $17 \mathrm{H}$ ), weakly brochidodromous (Figures 15I, 17F-G), or terminating in the intramarginal vein (Figures $15 \mathrm{H}, 17 \mathrm{~K}$ ). Sinus-bracing veins arise as a single thick secondary vein, 


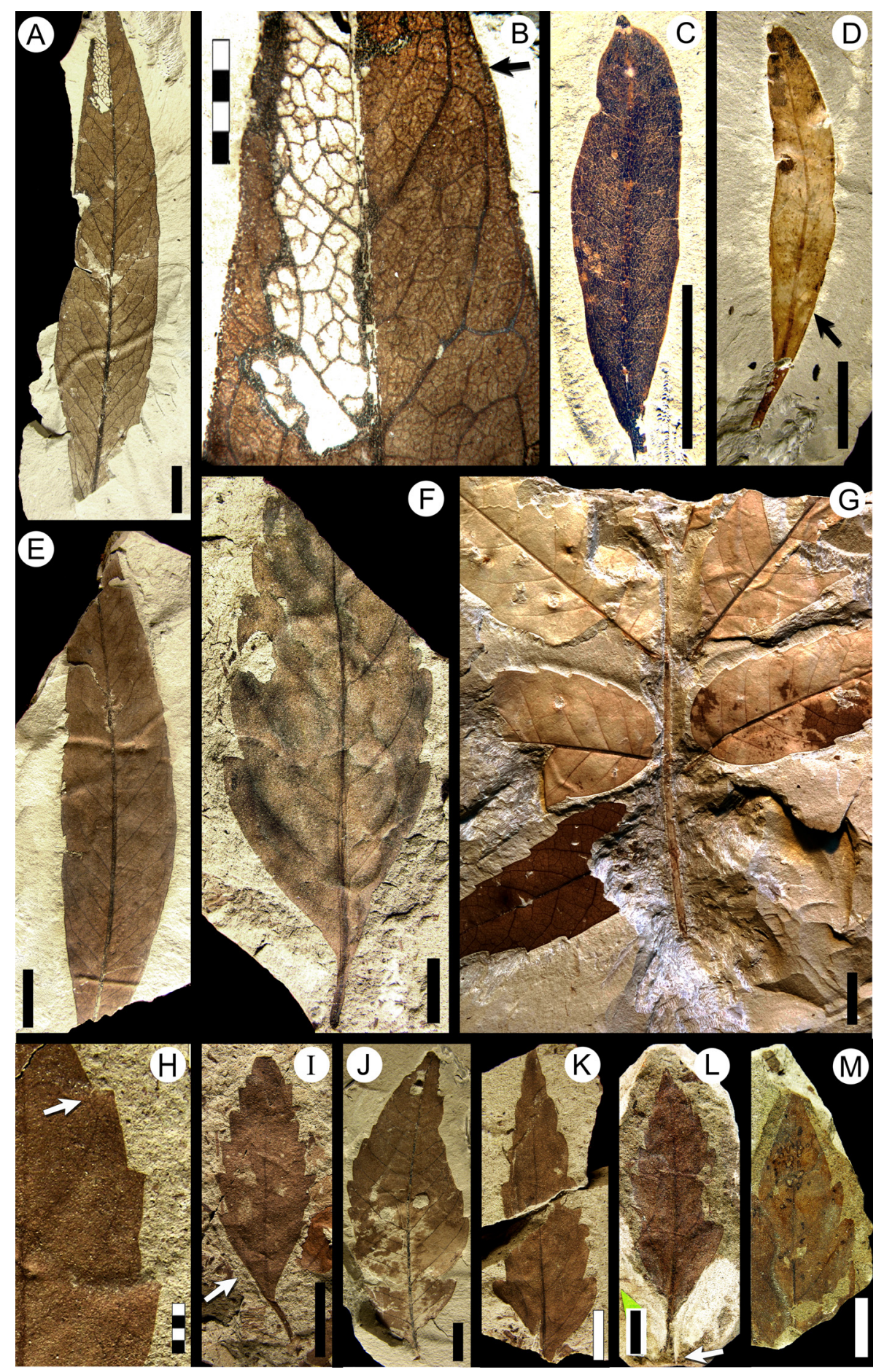

FIGURE 16. Morphotype SA016 (see also Figure 19F-I) and morphotype SA042 "Fagophyllum duseni" Berry 1937a (Cunoniaceae; see also Figure 25A-D). A-E, morphotype SA016. A, MPEF-Pb-2028 (exemplar). B, venation and skeletonization insect damage (DT19; see also Donovan et al., 2016) in apex detail of specimen in Figure 16A (arrow, intramarginal vein); C, MPEF-Pb-4062; D, MPEF-Pb-9067 (arrow, intramarginal vein); E, MPEF-Pb-9068. F-M, morphotype SA042, "F. duseni" (Cunoniaceae). F, MPEF-Pb-2035 (exemplar); G, articulated compound leaf MPEF-Pb9156; $\mathrm{H}$, tooth venation detail in MPEF-Pb-4093 (arrow, apically deflected vein from a secondary); I, MPEF-Pb-9070 (arrow, untoothed leaf base); J, MPEF-Pb-4098; K, MPEF-Pb-9069. L-M, "F. duseni” syntypes. L, USNM-201960 (illustrated in Berry 1937a, plate V, figure 1; see also Table 2) (arrow, swollen petiole base); M, USNM-201961 (illustrated in Berry 1937a, plate V, figure 2; see also Table 2). Single-color scale bars equal $10 \mathrm{~mm}$; grid scales equal one millimeter (per rectangle). 


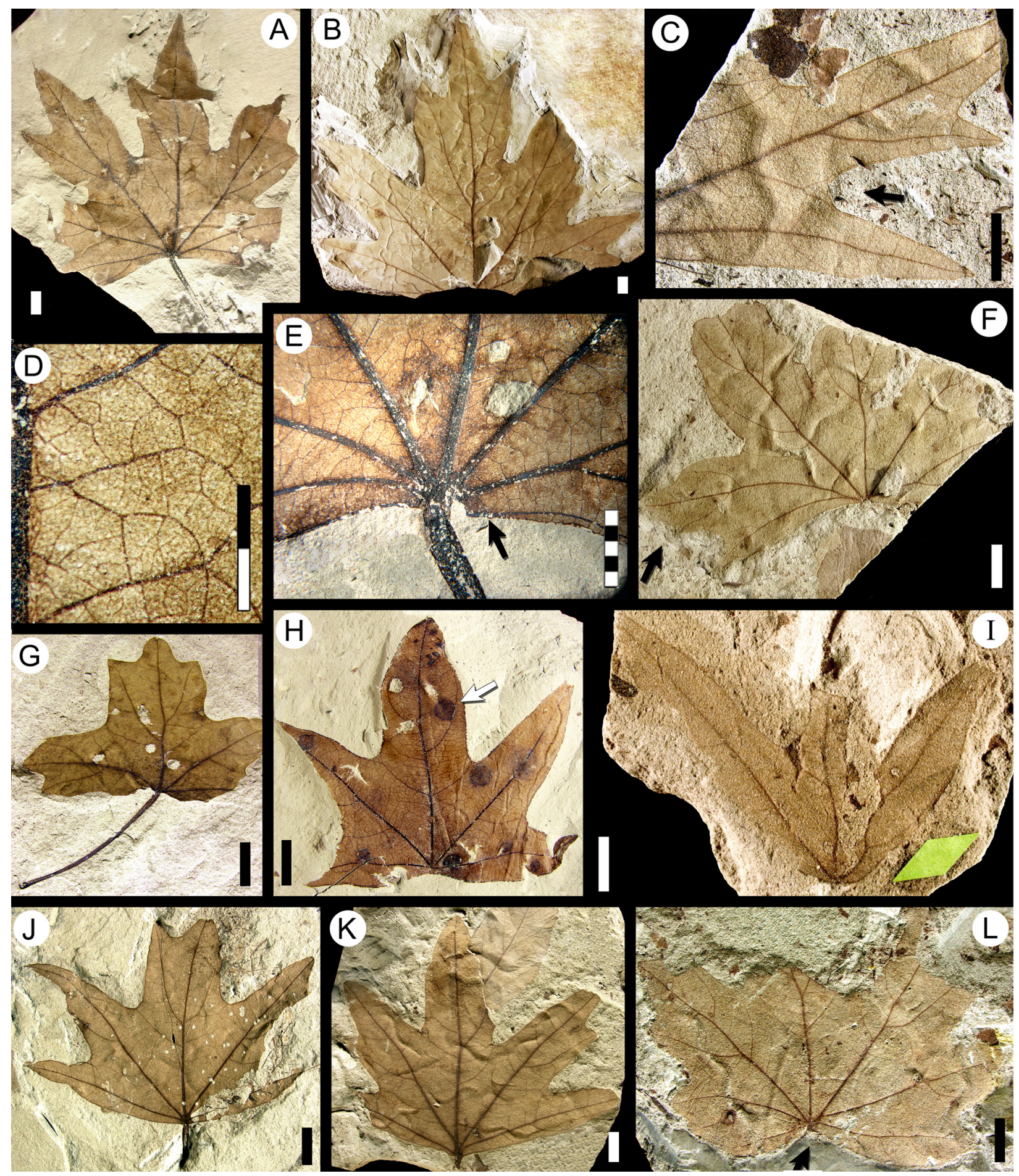

FIGURE 17. Morphotype SA019 (continued from Figure 15E-I). A, MPEF-Pb-2029 (exemplar, see also Figure 17D-E); $\mathrm{B}, \mathrm{MPEF}-\mathrm{Pb}-9149$, with Cochlichnus trace fossils; C, venation detail of MPEF-Pb-4070 (arrow, sinus-bracing vein); $\mathrm{D}$, minor venation detail from leaf in Figure 17A (exemplar); E, base detail of leaf in Figure 17A (exemplar; arrow, intramarginal vein); F, MPEF-Pb-9073 (arrow, secondary lobe; G, MPEF-Pb-9072; H, MPEF-Pb-4072, with several fungal pycnidia or stromata leaf-damage rings (DT334, arrow; see also Donovan et al., 2018, figure 10.13); I, "Sterculia" acuminataloba Berry, 1937a syntype USNM-208528 (illustrated in Berry 1937a, plate VIII, figure 5; see also Table 2); J, MPEF-Pb-4071; K, MPEF-Pb-9075; L, MPEF-Pb-9076 (arrow, cordate base). Single-color scale bars equal $10 \mathrm{~mm}$; grid scales equal one millimeter (per rectangle). 
course straight, then forking far from the margin and joining the intramarginal vein (Figures 15E, $15 G, 17 C)$. Intersecondary veins thin, simple, and short (Figure 15E). Perimarginal venation an irregular intramarginal vein. Intersecondary veins thin, proximally and distally parallel to subjacent secondary, shorter than half the length of the subjacent secondary, frequency one per secondary (Figure 15E). Intercostal tertiary venation opposite percurrent, perpendicular to secondaries, angles increasing exmedially (Figures 15E, 15G, 17I). Epimedial tertiary venation opposite percurrent; tertiaries thick, course straight or convex to chevroned, spacing irregular, departure from primaries nearly perpendicular (Figures 15H, 17D). Fourth and fifth order venation regular polygonal reticulate (Figure 15F, 17D). Areolation well developed, areoles fourfive sided (Figure 15F, 17D). Freely ending veinlets absent. Vein density $10.12 \mathrm{~mm} / \mathrm{mm}^{2}$ (one leaf measured). Marginal ultimate venation a thin intramarginal vein, running very close to the margin (Figure 15H-I).

Observations. Morphotype SA019 can be distinguished from other palmately lobed leaf morphotypes using a number of features including: lobes compound; base cordate; primary veins actinodromous, characteristically deflected by thick secondaries at the base of each lobe; secondary veins thick; and tertiary veins percurrent, chevroned towards base (see key in supplementary Appendix 2). Whereas the original description of "Sterculia" acuminataloba Berry, 1937 from Cerro Funes (Palacio de los Loros) matches morphotype SA014 as discussed above, syntype USNM-208528 (Figure 17I) does not show the same leaf morphology as the original description, instead corresponding to our morphotype SA019. As for SA014, there are many fossil leaf species from Patagonia that have been referred to Sterculia Linnaeus, 1753 that superficially resemble morphotype SA019, but none shows the same suite of morphological characters. Morphotype SA019 is present through all three Paleocene time intervals in this study (Table 1).

\section{Morphotype SA020}

Equivalent to. "Myrica premira" Berry, 1937 in part: syntype USNM-201962 (Berry, 1937a, plate $\mathrm{V}$, figure 3; here Figure 29C) only (see Table 2), USNM-201963 syntype excluded (see morphotype SA049).

Affinity. Cunoniaceae Brown, 1814 nom. cons.

Exemplar. MPEF-PB-2030 from quarry PL2 (Figure 18A, 18F).
Accessory exemplar. MPEF-PB-3020 from quarry PL2 (Figure 18G, 18K, 19E).

Figures 18, 19A-E

Description. Leaf odd pinnately compound with almost four pairs of alternate leaflets (Figure 18M). Leaflets petiolulate, medially asymmetric with asymmetric insertion; size nanophyll to mesophyll, generally microphyll. Mean length 7.6 (5.5-11.5) $\mathrm{cm}$, mean width $2.0(0.9-3.8) \mathrm{cm}$. Length:width ratio 5:1. Margin serrate. Petiolule insertion marginal (Figures 18L-M, 19B), semiterete in cross section. Texture chartaceous. Shape narrow ovate (Figure 18C-E, 18J-L). Apex shape extended acuminate (Figure 18A), angle acute. Base shape concave, angle acute, decurrent on the petiolule (Figure 18B-D). Primary venation pinnate. Midvein thick (Figure 18G), course smoothly curved. Basal veins one (Figure 19B-C). Agrophic veins absent. Secondary venation craspedodromous, becoming semicraspedodromous apically (Figures 18K-L, $19 \mathrm{E}$ ) and brochidodromous basally (Figures 18J-L, 19D). Secondary veins bifurcate near margin (Figure 19A-B, 19D) and sometimes join adjacent secondaries, forming loops (Figure 19E). Veins opposite to subopposite, course smoothly curved, regularly spaced, angle to midvein regular, departure from midvein excurrent. Perimarginal venation a thick, well-developed fimbrial vein (Figure 18F$\mathrm{G})$. Intersecondary veins simple, proximally and distally parallel to subjacent secondary, longer than half the length of subjacent secondary, angle to midvein broader than that of main secondary veins; less than one per secondary (Figure 19B, 19E). Intercostal tertiary venation alternate percurrent, develops in 4-5 rows of vein fields per intercostal area (Figures 18F-G, 19A, 19E). Epimedial tertiary venation mixed reticulate and percurrent, perpendicular to primary, course straight to sinuous, uniform angles (Figure 19A). Fourth order veins alternate percurrent (Figure 19E). Fifth order veins regular polygonal reticulate. Freely ending veinlets dichotomous branching (Figure 18G). Vein density range $6.78-14.36 \mathrm{~mm} / \mathrm{mm}^{2}$ (one leaf measured). Areolation well developed. Marginal ultimate venation join perimarginal vein. Teeth simple, triangular, well developed, and regular except at the leaf base, apex simple (Figure 18F-G). Tooth shape convex/convex, flexuous/convex, or flexuous/flexuous; sinuses rounded (Figure 18F). Tooth spacing uniform, one tooth per centimeter. Tooth venation supplied by a secondary vein running near the apical flank and reaching the tooth apex; and an apical short-curved branch running along the basal 


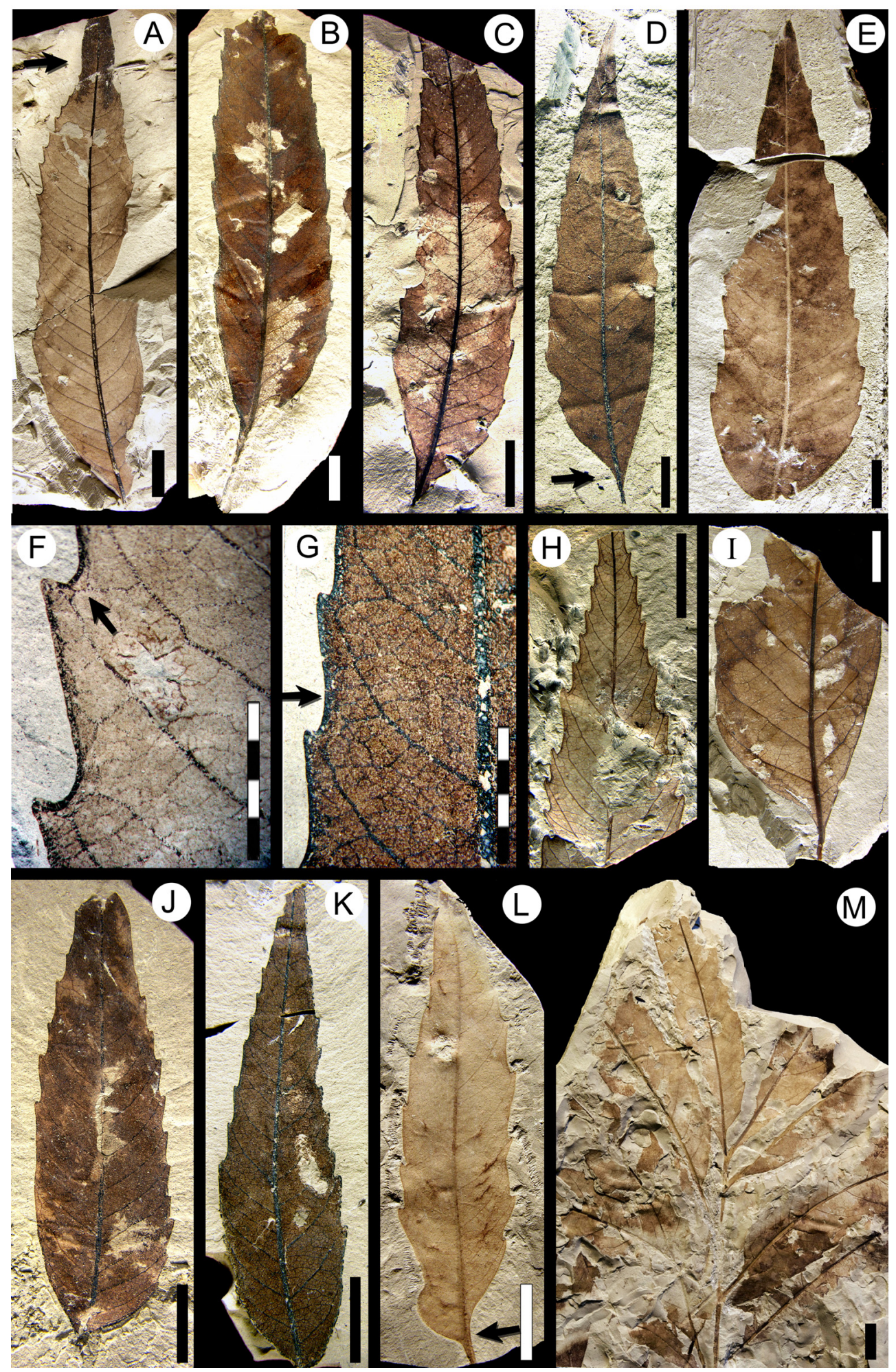

FIGURE 18. Morphotype SA020 (Cunoniaceae, see also Figure 19A-E). A, MPEF-Pb-2030 (exemplar, see also Figure 18F; arrow, extended acuminate apex); B, MPEF-Pb-9077; C, MPEF-PB-9078; D, MPEF-Pb-4076 (arrow, asymmetric decurrent base); E, MPEF-Pb-9079; F, perimarginal vein detail of specimen in Figure 18A (exemplar; arrow, an apical, short curved branch); G, tooth venation detail of specimen in Figure 18K (accessory exemplar; arrow, intramarginal vein); H, MPEF-Pb-9080; I, MPEF-Pb-9081; J, MPEF-Pb-9082; K, MPEF-Pb-3020 (accessory exemplar, see also Figure 18G); L, MPEF-Pb-9083 (arrow, asymmetric decurrent base); M, articulated compound leaf MPEF-Pb9154. Single-color scale bars equal $10 \mathrm{~mm}$; grid scales equal one millimeter (per rectangle). 

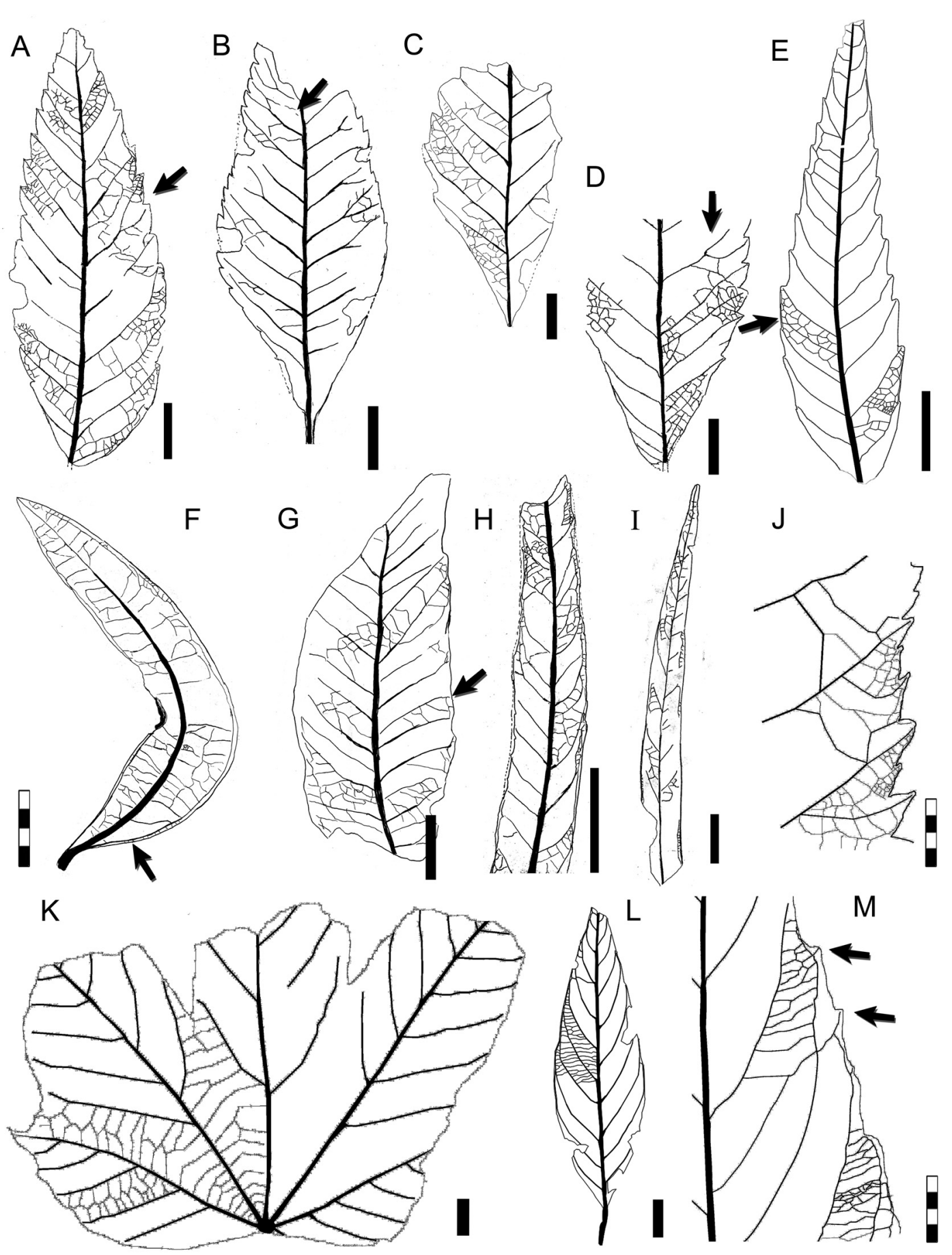

FIGURE 19. Morphotypes SA016 (continued, see also Figure 16A-E), SA035 (Bixaceae? see also Figure 20), SA020 (continued, see also Figure 18; Cunoniaceae), and SA041 (Rhamnaceae, see also Figure 23A-I). A-E, camera lucida drawings (CLD) of morphotype SA020 (Cunoniaceae). A, MPEF-Pb-9087 (arrow, bifurcation of a secondary vein); B, MPEF-Pb-9088 (arrow, intersecondary vein); C, MPEF-Pb-9089; D, MPEF-Pb-9090 (arrow, bifurcation of a secondary vein); E, MPEF-Pb-3020 (accessory exemplar, see also Figure 18K; arrow, intersecondary vein). F-I, CLD of morphotype SA016. F, MPEF-Pb-9091 (arrow, basal secondary vein); G, MPEF-Pb-9092 (arrow, intramarginal vein); $\mathrm{H}$, MPEF-Pb-9093; I, MPEF-Pb-9094. J-K, digital overlay drawing (DOD) of morphotype SA035 (Bixaceae?). J, tooth venation of MPEF-Pb-2031 (exemplar, see also Figure 20A, 20C); K, MPEF-Pb-4078. L-M, morphotype SA041 (Rhamnaceae). L, hand drawing of MPEF-Pb-2034 (exemplar, see also Figure 23A); M, DOD of venation in MPEF$\mathrm{Pb}-9095$ (arrow, two small, scattered teeth). Scale bars equal $10 \mathrm{~mm}$, except 4 millimeter scale in Figure 19F, 19J, and $19 \mathrm{M}$. 
flank of the supradjacent tooth; accessory tooth venation looped and fimbrial (Figure 18F-G).

Observations. All specimens of this morphotype have an asymmetric base and a smoothly curved primary vein, suggesting that they are isolated leaflets derived from compound leaves, and two specimens, MPEF-Pb-9154 (Figure 18M) and MPEF$\mathrm{Pb}-9155$, were found with organically attached leaflets. Morphotype SAO20 is found through all Danian time intervals considered here and is abundant from quarry PL2 (Table 1).

Morphotype SA020 matches the syntype USNM-201962 of "Myrica premira" (Berry, 1937a, plate V, figure 3; here Figure 29C), although it does not show the same characters listed in Berry's description and the other syntypes for that species (see morphotype SA049). USNM-201962 has an extended acuminate apex, craspedodromous secondary venation, a thick fimbrial vein, and regularly shaped teeth that correlate to our morphotype SA020 (Table 2).

The robust fimbrial vein, asymmetric base, craspedodromous secondary venation, and the tooth venation with an asymmetric midvein and a small apical branch (cunonioid tooth sensu Hickey and Wolfe, 1975) are consistent with Cunoniaceae. As mentioned earlier, Jud et al. (2018a) recently described cunoniaceous flowers (Tribe Schizomerieae) from the same localities of the Salamanca and Peñas Coloradas formations. Characters that distinguish this morphotype from similar cunonioid leaflets (i.e., SA005, SA042, SA043, SA44, SA049, SA054; Table 1) include: blade ovate; secondary veins forking far from margin to vascularize two teeth; intersecondaries weak, intramarginal vein thick, teeth triangular, and the tooth principal vein formed by a small branch that reaches the basal flank of the supradjacent tooth (see key in supplementary Appendix 2).

\section{Morphotype SA035}

Affinity. Bixaceae? Kunth, 1822 nom. cons. Exemplar. MPEF-PB-2031 from quarry PL1 (Figures 19J, 20A, 20C).

\section{Figures 19J-K, 20}

Description. Blade symmetric, microphyll to mesophyll, generally notophyll. Mean length 5.5 $(1.5-8.5) \mathrm{cm}$, mean width $6.0(1.4-11.0) \mathrm{cm}$. Length:width ratio (1:1-2:1). Margin serrate (Figure 19J). Petiole insertion marginal (Figure 20A, 20G). Texture chartaceous. Shape elliptic, unlobed (Figure 20E) or palmately 3-5 lobed (Figure 20A, 20G). Apex shape convex (Figure 20G, 20I), lobe-apex angle wide-obtuse. Base wide; shape concavo- convex (Figure 20A), cordate (Figure 20B, 20F, $20 \mathrm{I}$ ), or straight (Figure 20G); angle obtuse. Primary venation basal actinodromous with one primary vein per lobe, up to three thick primaries with straight courses and two thin laterals, for both the lobed and unlobed forms. In unlobed forms, lateral primaries taper apically, reaching the same strength as secondaries. Basal veins five, three primary veins and and two thinner lateral primaries (Figures 19K, 20B). Agrophic veins compound (Figure 20A, 20E, 20H). Secondary venation craspedodromous (may be semicraspedodromous at base, Figure 20F); veins robust, opposite to subopposite, course curved abruptly near the margin and branching to innervate teeth. Secondary spacing uniform, angle slightly decreasing toward base (Figure 20B, 20G-H). Sinus-bracing veins welldeveloped (Figure 20B, 20G). Perimarginal venation a thin fimbrial vein (Figures 19J, 20C). Intersecondary and interior secondary veins absent. Intercostal tertiary venation mixed opposite-alternate percurrent (Figure 19K). Epimedial tertiary venation alternate percurrent with perpendicular divergence from midvein, more or less uniform in gauge and course. Fourth and fifth order venation regular polygonal reticulate (Figure 19J). Vein density $10.59 \mathrm{~mm} / \mathrm{mm}^{2}$ (one leaf measured). Areolation moderately developed. Marginal ultimate venation looped (Figure 19J). Teeth compound in two orders, triangular, generally with a simple (Figures 20C, 19J) or spinose apex, sometimes with an opaque callosity fused to the apex (Figure 20F). Tooth shape concave/concave, concave/flexuous, straight/flexuous, straight/straight, or flexuous/flexuous; sinuses angular (Figures 20C, 19J). Tooth spacing slightly irregular, one to two teeth per centimeter. Tooth venation supplied by a robust medial vein arising from a secondary or a secondary branch, emitting a minor branch that innervates the supradjacent sinus; accessory veins looped and merging with the fimbrial vein (Figure 19J).

Observations. Morphotype SA035 is present at low abundances through all three Danian time intervals considered here (Table 1). It differs from morphotype SA009 (in part = "Cissites" patagonica Berry, 1937a) in base shape, tooth type, interior secondaries, and second- and higher-order venation (see key in supplementary Appendix 2). The teeth of morphotype SA035 can be assigned to the violoid type following Hickey and Wolfe (1975). Morphotype SA035 shares several features with extant Cochlospermum Kunth, 1822 nom. cons. (Bixaceae), such as symmetrical, palmately veined leaves with craspedodromous secondary venation 


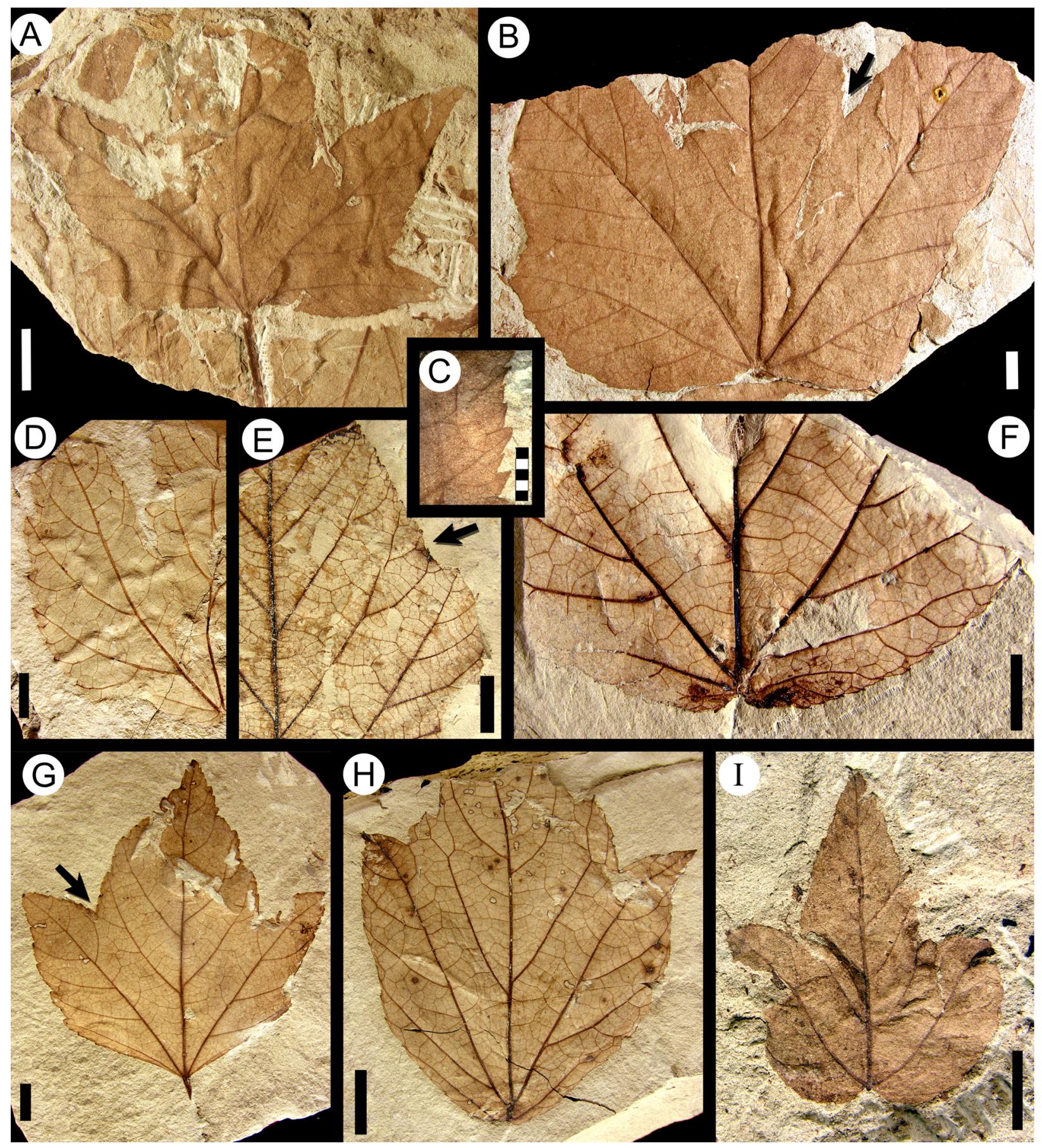

FIGURE 20. Morphotype SA035 (Bixaceae? continued, see also Figure 19J-K). A, MPEF-Pb-2031 (exemplar, see also Figures 19J, 20C); B, MPEF-Pb-4078 (arrow, sinus-bracing vein); C, tooth detail of specimen in Figure 20A (exemplar); D, venation detail at leaf base, MPEF-Pb-9096; E, venation detail of MPEF-Pb-9097 (arrow, compound agrophic veins); F, basal venation of MPEF-Pb-9098; G, MPEF-Pb-4083 (arrow, sinus-bracing vein); H, MPEF-Pb4082; I, MPEF-Pb-4079. Scale bars equal $10 \mathrm{~mm}$, except 5 millimeter scale in Figure 20C. 
and no intersecondaries, tertiary veins perpendicular to primary vein, presence of simple and compound teeth with angular sinuses, and looped marginal ultimate venation. Cochlospermum previtifolium Berry, 1935a leaves and fruits are known from the Eocene Laguna del Hunco and Río Pichileufú floras (Berry, 1935a; González, 2008), and that taxon shares the characters noted here for the extant species. C. previtifolium differs from morphotype SA035 in having a straight/convex tooth shape, percurrent tertiary venation, and a thick fimbrial vein.

\section{Morphotype SA038}

Affinity. Unknown.

Exemplar. MPEF-Pb-3033 from quarry OR2 (Figures 21C, 22I).

Accessory exemplar. MPEF-Pb-3034 from quarry OR2.

Figures 21C, 22I

Description. Blade simple, symmetric, notophyll to mesophyll, generally mesophyll. Mean length 7.2 $(4.8-8.2) \mathrm{cm}$, mean width $9.0(7.0-10.5) \mathrm{cm}$. Mean length:width ratio 0.8:1 (0.7:1-0.9:1). Margin entire. Petiole insertion marginal or subpeltate (Figure 21C). Shape broad elliptic in outline, medially and basally symmetrical, palmately 3-lobed, lobes wide and shallow (Figure 22I). Lobe apex convex; lobe sinuses rounded, narrow but shallow. Apex angle obtuse. Base shape lobate with a marked basal sinus, angle reflexed. Primary venation basal palinactinodromous, course straight (Figure 22I). Basal veins five, three palinactinodromous primaries and two thinner basal secondaries (Figure 21C). Agrophic veins simple, moderate in thickness. Secondary venation brochidodromous. Secondary veins thick, origination subopposite, course curved, joining supradjacent secondaries in a series of wide loops; spacing and angle smoothly decreasing basally; departure from midvein excurrent (Figure 21C). Perimarginal venation absent. Intersecondary veins proximally perpendicular to midvein, shorter than half the length of the subjacent secondary, distally reticulating or ramifying; one per secondary. Intercostal tertiary venation opposite percurrent, departing from secondaries at right angles. Epimedial tertiary venation (could be considered interior secondaries) opposite percurrent, moderately thick, course straight; departure from primaries perpendicular, opposite at the base; angle increasing exmedially, forming a concentric 'spider web' pattern at the base (Figure 21C). Fourth and fifth order venation regular polygonal reticulate. Freely ending veinlets unbranched. Are- olation well developed, areoles four-sided. Marginal ultimate venation looped.

Observations. Morphotype SA038 is only known from quarry OR2 (Table 1). It differs markedly from other lobed morphotypes (e.g., morphotype SA039) in exhibiting palinactinodromous primary venation, brochidodromous secondary venation, a lobate base, and five basal veins.

\section{Morphotype SA039}

Equivalent to. "Paranymphaea" aristolochiaformis Berry, 1937 (holotype USNM-201964; Berry, 1937a, plate $\mathrm{V}$, figure 5; here Figure $22 \mathrm{~A}$ and redrawn in Figure 21A, see also Table 2).

Affinity. Unknown.

Exemplar. MPEF-Pb-2032 from quarry PL1 (Figures 21B, 22B, 22D).

\section{Figures 21A-B, 22A-H}

Description. Blade simple, symmetrical; size notophyll to mesophyll, most frequently mesophyll. Maximum length to $15 \mathrm{~cm}$, maximum width to 18 $\mathrm{cm}$. Length:width ratio $\sim 0.8: 1$ (one leaf measured). Margin entire. Petiole insertion marginal or subpeltate (Figure 22G-H), disposed nearly perpendicular to the plane of the blade. Shape ovate, palmately 5-lobed; lobes wide and shallow (Figure 22G-H), lobe sinuses rounded (Figure 21B); lobe apex convex (Figure 22E) or acuminate (Figure 22B), apex angle obtuse. Base shape lobate, basal sinus prominent, base reflex (Figure 22A-E, 22G-H). Apex shape convex (Figure 22B, 22G), lobe-apex angle obtuse. Primary venation basal actinodromous; primary veins moderate in thickness, course deflected, occasionally forked near the margin (Figure 21A). Basal veins seven to nine, with five to seven primaries and two thinner basal secondaries (Figures 21A-B, 22D). Agrophic veins simple or compound, festooned brochidodromous (Figure 21B). Secondary venation festooned brochidodromous; secondaries thick, subopposite, course straight or abruptly curved; departure from midvein excurrent, deflecting the midvein. Secondary spacing increasing toward base; angle smoothly decreasing toward base. Sinus-bracing veins poorly marked, forking far from the sinus (Figure 21B). Perimarginal and intersecondary veins absent. Intercostal tertiary venation alternate percurrent, thickness moderate (Figures 21A-B, 22F). Epimedial tertiary venation opposite percurrent, course straight, opposite toward base of blade, otherwise alternate, perpendicular to primary, tertiary angle increasing exmedially, forming a concentric 'spider web' pattern (Figures 21A, 22D). Fourth and fifth order venation regular polygonal 


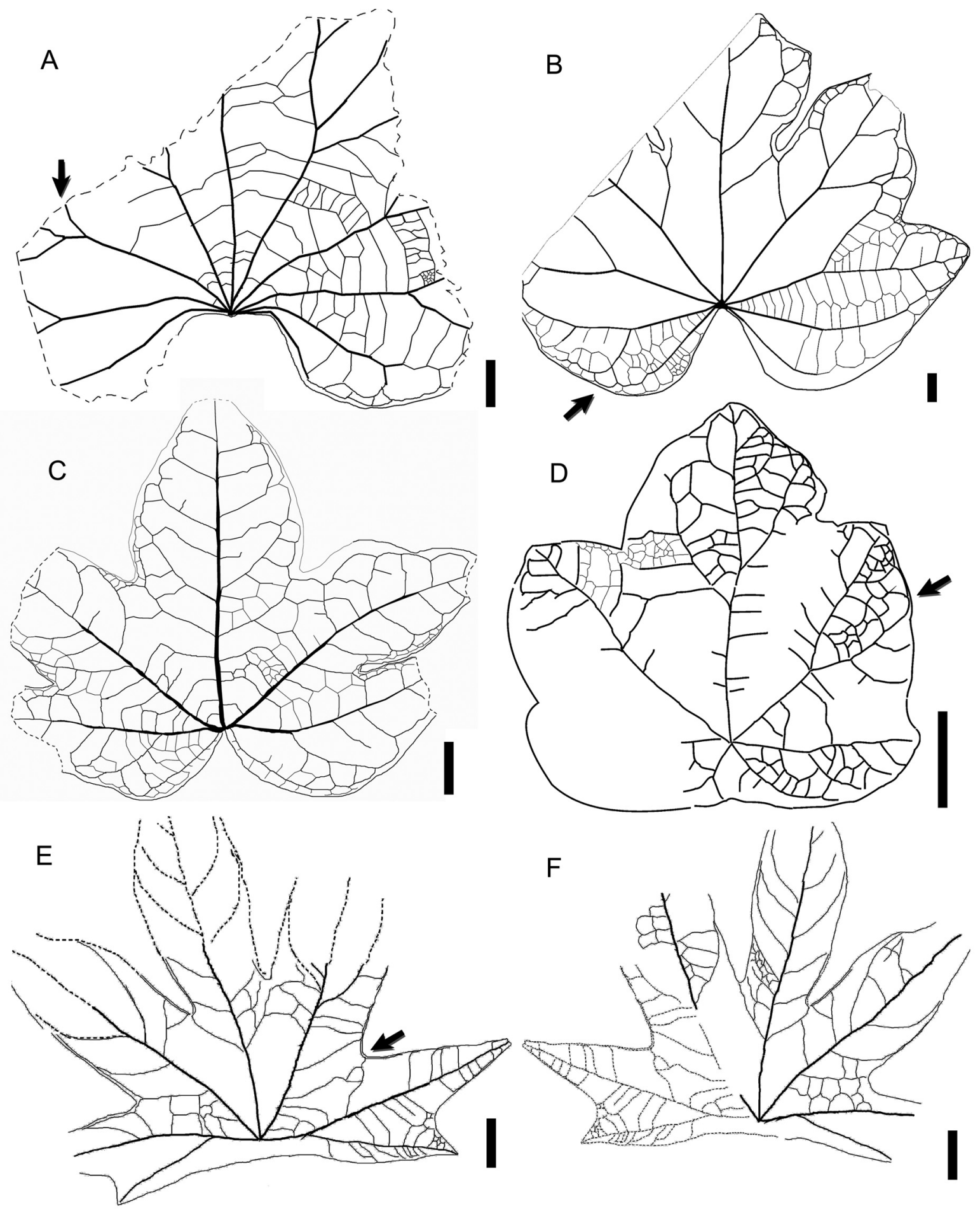

FIGURE 21. Morphotypes SA038 (see also Figure 22I), SA039 "Paranymphaea" aristolochiaformis Berry 1937a (see also Figure 22A-H), SA040 (see also Figure 22J-M), and SA055 (see also Figure 30K-L). A-B, drawings of morphotype SA039. A, redrawing of "Paranymphaea" aristolochiaformis syntype USNM-201964 (illustrated in Berry, 1937a, plate $\mathrm{V}$, figure 5; see also Figure $22 \mathrm{~A}$ and Table 2; arrow, primary vein distally forking); B, digital overlay drawing (DOD) of MPEF-PB-2032 (exemplar, see also Figure 22B, 22D; arrow, festooned brochidodromous agrophic veins). C, DOD of morphotype SA038, MPEF-Pb-3033 (exemplar, see also Figure 22I). D, DOD of morphotype SA040, MPEF-Pb-2033 (exemplar; see also Figure 22J; arrow, forked lateral primary vein). E-F, DOD of morphotype SA055, MPEF-PB-2047 (exemplar, part and counterpart; see also Figure 30K-L), counterpart reconstructed areas dotted (arrow, sinus-bracing and intramarginal veins). Scale bars equal $10 \mathrm{~mm}$. 

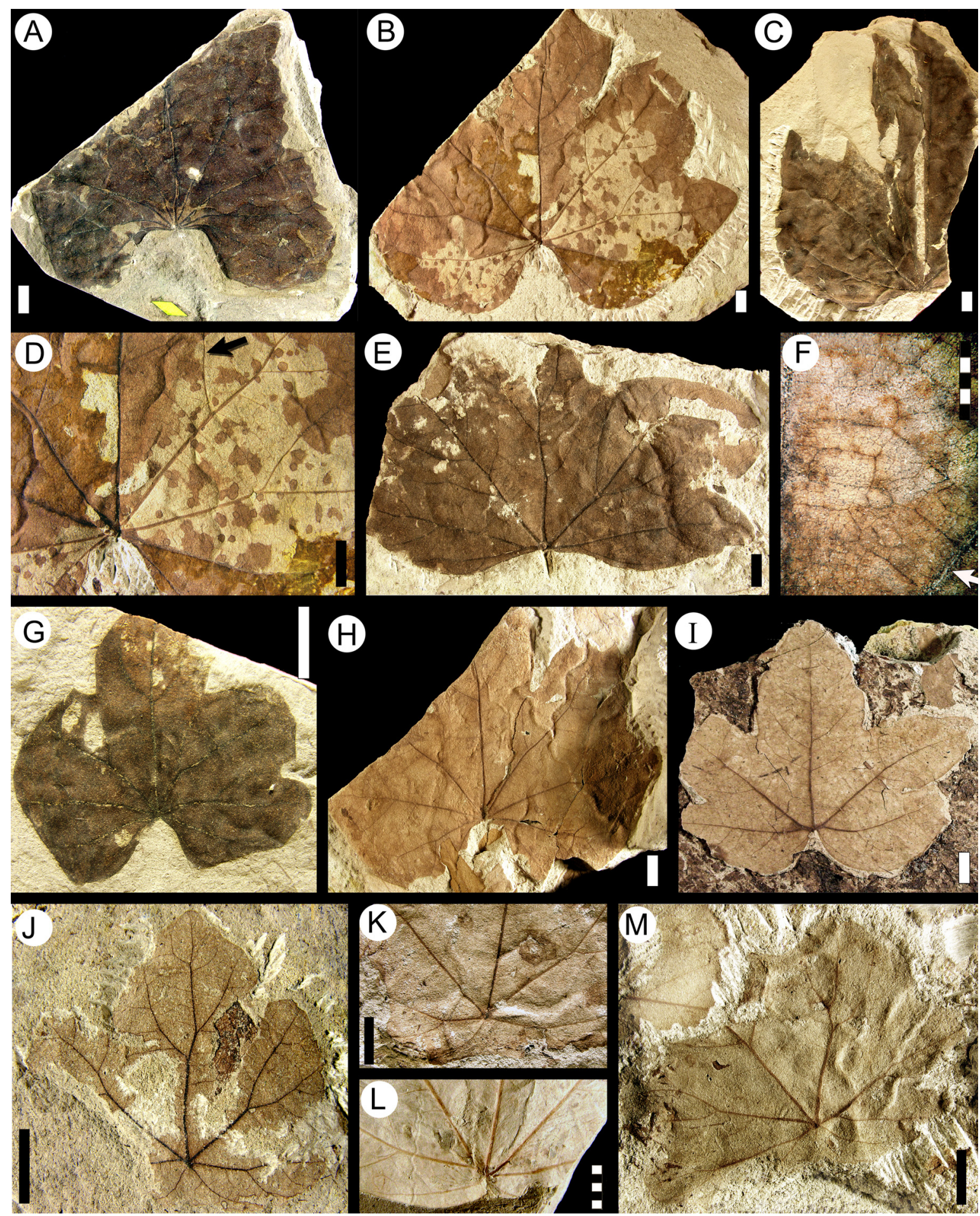

FIGURE 22. Morphotypes SA038 (continued, see also Figure 21C), SA039 "Paranymphaea" aristolochiaformis Berry 1937a (continued, see also Figure 21A-B), and SA040 (continued, see also Figure 21D). A-H, morphotype SA039. A, "P." aristolochiaformis syntype USNM-201964 (illustrated in Berry, 1937a, plate V, figure 5; see also Figure 21A and Table 2); B, MPEF-Pb-2032 (exemplar, see also Figures 21B, 22D); C, MPEF-Pb-9099; D, venation at leaf base of specimen in Figure 22B (exemplar; arrow, secondary vein); E, MPEF-Pb-9100; F, MPEF-Pb-4084 (arrow, primary vein); G, MPEF-Pb-9095; H, MPEF-Pb-9101. I, morphotype SA038, MPEF-Pb-3033 (exemplar, see also Figure 21C). J-M, morphotype SA040. J, MPEF-Pb-2033 (exemplar, see also Figure 21D); K, peltate base of MPEF-Pb-9102; L, peltate base of MPEF-Pb-9103; M, MPEF-Pb-9104. Scale bars equal $10 \mathrm{~mm}$, except 5 millimeter scale in Figure 22F and 22L. 
reticulate (Figure 21A). Vein density $9.74 \mathrm{~mm} / \mathrm{mm}^{2}$ (one leaf measured). Areolation well developed, areoles four-sided. Freely ending veinlets unbranched. Marginal ultimate venation looped (Figure 21B).

Observations. Morphotype SA039 has characters identical to those of the type specimen of "Paranymphaea" aristolochiaformis Berry, 1937 collected at the Palacio de los Loros locality (Berry, 1937a). More and better-preserved material here allows more complete description of this species, which Berry (1937a) described as palmate, even though most of the leaf margin, shape, and apex were not preserved in Berry's material (Figure 22A). The new material shows that the leaf is lobate rather than palmate. Paranymphaea Berry, 1935 is a fossil genus proposed by Berry (1935b) for specimens from the Paleocene of the Northern Hemisphere, originally assigned to the Nymphaeaceae Salisbury, 1805. Morphotype SA039 has actinodromous primary venation and festooned brochidodromous secondary venation, characters that can be observed within Nymphaeaceae. However, in the family leaves with festooned brochidodromous venation also have several orders of loops progressing toward the margin. In addition, the new specimens of morphotype SA039 have a palmately 5-lobed, lobate base (not cordate or sagittate), robust secondary veins arising at acute angles, and dense agrophic veins, none of which occurs in living Nymphaeaceae. Morphotype SA039 was found at quarries PL1 and PL4 (Salamanca Formation) and at quarry LF (Peñas Coloradas Formation), at relatively low abundance (Table 1 ). It differs markedly from other lobate morphotypes (e.g., morphotype SA038) in its actinodromous primary venation, lobate base shape, and larger number of basal veins.

\section{Morphotype SA040}

Affinity. Unknown.

Exemplar. MPEF-Pb-2033 from quarry PL1 (Figures 21D, 22J).

Figures 21D, 22J-M

Description. Blade simple, symmetrical, size microphyll to notophyll, generally notophyll. Maximum length $5 \mathrm{~cm}$, maximum width $8 \mathrm{~cm}$. Length:width ratio $\sim 1: 1$ (one leaf measured). Margin entire, wavy. Texture membranaceous. Petiole insertion peltate eccentric (Figures 21D, 22J, 22M). Shape ovate, palmately lobed with five lobes. Lobes very shallow (incised less than $1 \mathrm{~cm}$ in length) and wide; lobe apex convex, lobe sinuses angular (Figure 22J). Apex angle obtuse. Base shape slightly cordate, base angle reflex (Figures 21D, 22J-K). Primary venation basal actinodromous, course deflected by secondaries, forking prominently toward the margin (Figure 22J). Lateral primaries disposed at $>180^{\circ}$ (Figure 21D). Basal veins six to seven, five primaries and one or two basal secondaries. Agrophic veins simple, generating brochidodromous loops (Figure 21D). Secondary venation brochidodromous. Secondary veins alternate to subopposite, thin, deflecting the primaries, spacing irregular, angle acute and irregular (Figure 21D). Perimarginal and sinus-bracing veins absent. Intercostal tertiary venation opposite percurrent, departing from secondaries at acute angles. Epimedial tertiary venation thin, opposite percurrent, course straight, tertiary angle to primary perpendicular at departure, then obtuse, at the base arranged in chevrons, tertiary vein angle increasing exmedially (Figure 21D). Fourth order veins regular polygonal reticulate, very thin. Marginal ultimate venation looped.

Observations. Morphotype SA040 differs from other palmately-lobed, entire margined leaves studied here (i.e., SA014, SA019, SA038, SA039, SA041, SA055, and SA58) in having wide and shallow lobes, petiole insertion peltate eccentric, and seven basal veins (see key in supplementary Appendix 2). Morphotype SA040 occurs at low abundance at the Palacio de los Loros locality (PL1 and PL2 quarries, Table 1).

\section{Morphotype SA041}

Affinity. Rhamnaceae Jussieu, 1789.

Exemplar. MPEF-Pb-2034 from quarry PL2 (Figures 19L, 23A, 23I).

Figures 19L-M, 23A-I

Description. Blade symmetric, size microphyll to mesophyll, generally notophyll. Mean length 8.8 $(5.0-11.4) \mathrm{cm}$, mean width $3.7(2.0-6.6) \mathrm{cm}$. Length:width ratio 2.5:1 (one leaf measured). Margin wavy (undulate, Figure 23G-H), irregularly crenate near secondary loops. Small teeth rarely present on some specimens (Figures 19M, 23G). Texture typically membranaceous. Petiole insertion marginal (Figure 23A, 23D). Shape ovate or elliptic. Apex shape acuminate, generally extending into a drip tip (Figure 23C, 23E), angle acute. Base shape convex (Figure 23A-E), angle acute. Primary venation pinnate, primary vein course straight or slightly sinuous (Figure 23D-E). Basal veins one. Secondary venation eucamptodromous (Figures 19L, 23H); secondaries opposite to alternate on the midvein in 10-12 pairs, course regularly curved; spacing uniform, angle acute and uniform 


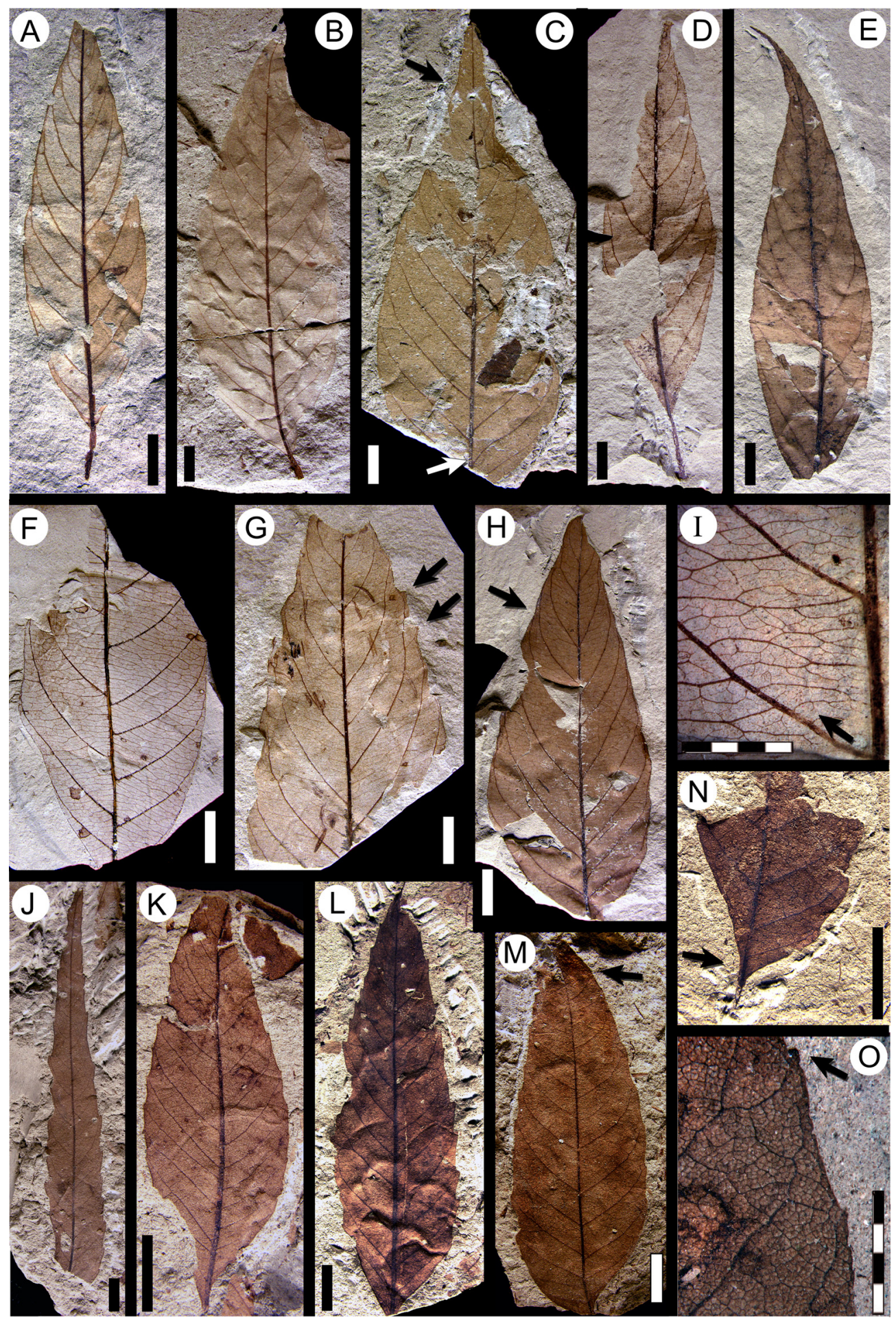

FIGURE 23. Morphotypes SA041 (Rhamnaceae; continued, see also Figure 19L-M) and SA044 (Cunoniaceae, see also Figure 25G-H). A-I, morphotype SA041 (Rhamnaceae). A, MPEF-Pb-2034 (exemplar, see also Figure 19L); B, MPEF-Pb-4085; C, MPEF-Pb-4089 (arrow, decurrent secondary veins); D, MPEF-Pb-4090; E, counterpart of MPEFPb-4090; F, MPEF-Pb-9105; G, MPEF-Pb-9106 (arrows, scattered teeth); H, MPEF-Pb-9107 (arrow, wavy margin); I, minor venation detail from leaf in Figure 23A (arrow, one-branched freely ending veinlets). J-O, morphotype SA044 (Cunoniaceae). J, MPEF-Pb-4105; K, MPEF-Pb-2037 (exemplar, see also Figure 25G-H); L, MPEF-Pb-3024 (accessory exemplar); M, MPEF-Pb-9108 (arrow, apical brochidodromous secondary vein); N, asymmetric base (arrow) of MPEF-Pb-9109; O, tooth venation of specimen in Figure 23K (exemplar; arrow, small triangular tooth). Scale bars equal $10 \mathrm{~mm}$, except 4 millimeter scale in Figure $23 \mathrm{l}$ and 230. 
(Figure 23A); departure from midvein decurrent for proximal secondaries, otherwise excurrent (Figure 23B-E). Perimarginal, agrophic and intersecondary veins absent. Intercostal tertiary venation markedly opposite percurrent (Figures 19L, 23F), departure from secondaries perpendicular. Epimedial tertiary venation mixed opposite-alternate percurrent (Figure 23I), course sinuous, disposed densely (more than 25 per intercostal area), very thin, departure from primary perpendicular, then obtuse at a uniform angle (Figures 19L-M, 23F). Fourth order veins regular pentagonal reticulate. Fifth order veins dichotomizing (Figure 23I). Vein density range $8.27-8.68 \mathrm{~mm} / \mathrm{mm}^{2}$. Areolation poorly developed. Freely ending veinlets one-branched. Marginal ultimate venation looped. Teeth irregularly spaced and scattered (Figures 19M, 23G). Tooth venation from secondary loops (Figure 19M).

Observations. Based on the wavy margin, pinnate primary venation, eucamptodromous secondary venation, and dense tertiaries that are disposed perpendicular to the primary and at homogeneous angles, morphotype SA041 could be assigned to Rhamnaceae Jussieu, 1789 (e.g., Hickey and Wolfe, 1975). Morphotype SA041 was found at quarries PL1, PL2 and PL4 (Salamanca Formation), with relatively high abundance at quarry PL2 (Table 1). Jud et al. (2017) described fossil flowers and leaves related to the ziziphoid/ampelozizyphoid clades of Rhamnaceae Jussieu, 1789 from the Rancho Grande (RG) locality (Chron C29n, Salamanca Formation, Figure 1); however, those leaves are quite different from morphotype SA041, which is more similar to other groups of Rhamnaceae such as Sageretia Brongniart 1826, from the rhamnoid clade (Richardson et al., 2000). Distinguishing characters of this morphotype include: margin wavy, irregularly crenate near secondary loops; a drip tip; and 10-12 pairs of regular, eucamptodromous secondary veins (see key in supplementary Appendix 2). Rhamnidium preglabrum Berry, 1925a (Rhamnaceae) from the Eocene Río Pichileufú and Laguna del Hunco (Berry, 1925a, 1938; Traverso, 1951) floras is probably the most similar Patagonian fossil leaf species to morphotype SA041. However, the Eocene material differs from morphotype SA041 in having a thicker and more prominent midvein and 8-11 pairs of secondary veins that are more thick and regular.

\section{Morphotype SA042}

Equivalent to. Fagophyllum duseni Berry, 1937 syntypes USNM-201960 (Berry, 1937a, plate V, figure 1; here Figure 16L), USNM-201961 (Berry, 1937a, plate V, figure 2; here Figure 16M); and "Dryophyllum australis" Berry, 1937 syntype USNM-208522 (Berry, 1937a, plate VI, figure 5; here Figure 7A) only (see Table 2); USNM-208523 syntype excluded (see morphotype SA004).

Affinity. Cunoniaceae Brown, 1814 nom. cons. Exemplar. MPEF-Pb-2035 (Figure 16F).

\section{Figures 16F-M, 25A-D}

Description. Leaf organization even-pinnately compound (Figure 16G), with at least three pairs of opposite leaflets. Rachis robust. Leaflet blade and base asymmetrical (Figure 16F-G, 16J-L), size microphyll to notophyll, generally microphyll. Mean length 6.2 (3.0-9.5) $\mathrm{cm}$, mean width 2.2 (1.1-4.0) $\mathrm{cm}$. Length:width ratio (2.1:1-4.1:1). Margin serrate. Petiolule short, insertion marginal, base slightly swollen (Figure 16G, 16L). Shape elliptic or ovate. Apex shape convex, angle acute (Figures 16J-M, 25A). Base shape straight, angle acute (Figure 16G, 16I-L). Primary venation pinnate, midvein thick, course straight or slightly curved. Basal veins one. Agrophic veins absent. Secondary venation craspedodromous. Secondary veins in seven to nine pairs, uniformly curved and regularly spaced; angle slightly irregular, increasing toward base, entering the teeth medially or apically. Secondaries generally not branching; if branched (Figure 25B-C) the apical branch runs toward the supradjacent basal tooth flank. Perimarginal venation a thin fimbrial vein originating from the base, thickenening the margin (Figure 16F). Intersecondary vein present, less than one per secondary (Figure 25B, 25D). Intercostal tertiary venation mixed opposite-alternate percurrent, departure from secondaries obtuse. Epimedial tertiary venation thin, percurrent or reticulate, course convex. Tertiary angle to midvein obtuse, uniform (Figure 25B). Fourth and fifth order veins regular polygonal reticulate. Areolation moderately developed. Marginal ultimate venation looped. Teeth simple asymmetric, non-glandular, large (length $1.6 \mathrm{~cm}$, width 0.6 $\mathrm{cm}$ ) and increasing in size toward the base of the blade (Figures 16F, 16I-L, 25A). Tooth shape convex/convex or straight/convex; sinuses angular (Figure 16H). Tooth spacing uniform, one per centimeter (Figure 16F), absent in the base of blade (Figure 16I-L). Tooth venation supplied by a secondary vein that enters asymmetrically into the tooth, plus an apical deflected vein running near the supradjacent tooth basal flank and reaching the tooth apex (Figures 16H, 25A-D).

Observations. Morphotype SA042 is present through all three Danian time intervals considered here (Table 1). It is equivalent to Fagophyllum 
duseni, which was described from two specimens from Palacio de los Loros (Figure 16L-M). Berry (1937a) assigned this material to the genus Fagophyllum Nathorst, 1888 because he found similarities with the genera Fagus Linnaeus, 1753 and Nothofagus Blume, 1851 nom. cons. (then considered part of Fagaceae). In addition, one of Berry's (1937a) syntypes of "Dryophyllum" australis Berry, 1937 (USNM-208522, Figure 7A) shows different leaf architecture from Berry's original description (see morphotype SA004) and is here considered equivalent to morphotype SA042 (Table 2).

The slightly asymmetric base and slightly curved primary vein of morphotype SA042 suggest that it corresponds to leaflets from compound leaves, confirmed by one specimen (MPEF-Pb9156, Figure 16F) found with organically attached leaflets. The compound leaf, asymmetric leaflet base, craspedodromous secondary venation, and the tooth venation with an asymmetric principal vein and a small apical branch (cunonioid tooth per Hickey and Wolfe, 1975) could place morphotype SA020 in the Cunoniaceae, also known from reproductive material from the same sites (Jud et al., 2018a; see Introduction).

\section{Morphotype SA043}

Affinity. Cunoniaceae? Brown, 1814 nom. cons. or Sapindaceae? Jussieu, 1789 nom. cons.

Exemplar. MPEF-Pb-2036 from quarry PL2 (Figure 24A).

Accessory exemplars. MPEF-Pb-3023 from quarry PL2 (Figure 24B), and MPEF-Pb-9110 from quarry PL2 (Figure 24C).

Figures 24A-G, 25E-F

Description. Leaf organization odd once-pinnately compound with at least three opposite, sessile to petiolulate leaflets (Figure 24C, 24G). Leaflets symmetric, size nanophyll to notophyll, generally microphyll. Mean length $4.2(2.8-5.7) \mathrm{cm}$, mean width 2.5 (1.3-3.7) $\mathrm{cm}$. Mean length:width ratio 2.4:1 (1.3:1-3.5:1); leaflet shape ovate or elliptic (Figure 24C, 24G). Texture chartaceous to membranaceous, characteristically preserved in a whitish yellow color (Figure 24A-E). Margin dentate. Petiolule sessile to $\sim 2.5 \mathrm{~cm}$ (on a terminal leaflet) in length, with an abscission zone at the base; insertion marginal (Figure 24C). Shape elliptic. Apex shape straight or concave (acuminate), angle acute (Figure 24B, 24E, 24G). Base asymmetric, shape cuneate, angle acute (Figure 24G). Primary venation pinnate; midvein thin $(0.7 \mathrm{~mm}$.), course straight. Basal veins one. Secondary venation craspedodromous; secondary vein course straight, spacing smoothly increasing toward base. Secondaries opposite on the midvein, arise at regular acute angles. Secondary veins fork once or twice near the margin, entering teeth medially (Figures 24F, 25E). Perimarginal and intersecondary veins absent. Intercostal tertiary venation mixed opposite-alternate percurrent, departure angle from secondaries obtuse (Figure 25F). Epimedial tertiary venation mixed opposite-alternate percurrent, course convex, departure angle from primary perpendicular and uniform (Figure 25E). Fourth order veins thick, regular polygonal reticulate. Fifth order veins regular polygonal reticulate, tending to opposite percurrent, forming large four-five sided areoles of $1.2 \mathrm{~mm}$ width (Figure 25E-F). Sixth order veins regular polygonal reticulate. Freely ending veinlets one-branched. Vein density $6.70 \mathrm{~mm} / \mathrm{mm}^{2}$ (one leaf measured). Areolation well developed (Figure 24F). Marginal ultimate venation looped. Teeth well developed around whole blade, simple, in two orders (Figure 25F). Teeth large (length 2.7 $\mathrm{mm}$, width $3.0 \mathrm{~mm}$ ), apex glandular to spherulate (Figures 24F, 25F). Tooth shape concave/flexuous, straight/flexuous, straight/concave, or flexuous/ flexuous; sinuses angular. Tooth spacing uniform, two teeth per centimeter (Figure 25E). Tooth venation supplied from secondary veins entering the first-order teeth medially and reaching the apex, minor secondary branches entering the subjacent second-order teeth, and apical secondary branches entering the superjacent sinuses (Figures $24 \mathrm{~F}, 25 \mathrm{~F}$ ).

Observations. Distinguishing characters of morphotype SA043 include: blade elliptic; secondary veins forking; perimarginal and intersecondary veins absent; and teeth prominent and in two orders (see key in supplementary Appendix 2). Morphotype SA043 is present through all three Danian time intervals considered here (Table 1). It is a compound leaf, as demonstrated by several attached specimens (e.g., Figure 24C, 24G) and resembles some genera in Cunoniaceae (e.g., Acrophyllum Bentham in Maund, 1840; Callicoma Andrews, 1809; and Vesselowskya Pampanini, 1905). In Cretaceous-Paleogene floras from southern South America, many fossil leaflets similar to morphotype SA043 have been placed in the genus Cupania Linnaeus, 1753 (Sapindaceae) (Engelhardt, 1891; Berry, 1925a, 1938; Traverso, 1951; Hünicken, 1967, 1995; Troncoso, 1992; Troncoso et al., 2002); however, the presence of that genus has never been confirmed with modern methods, and no extant species of Cupania resembles morphotype SA043. 


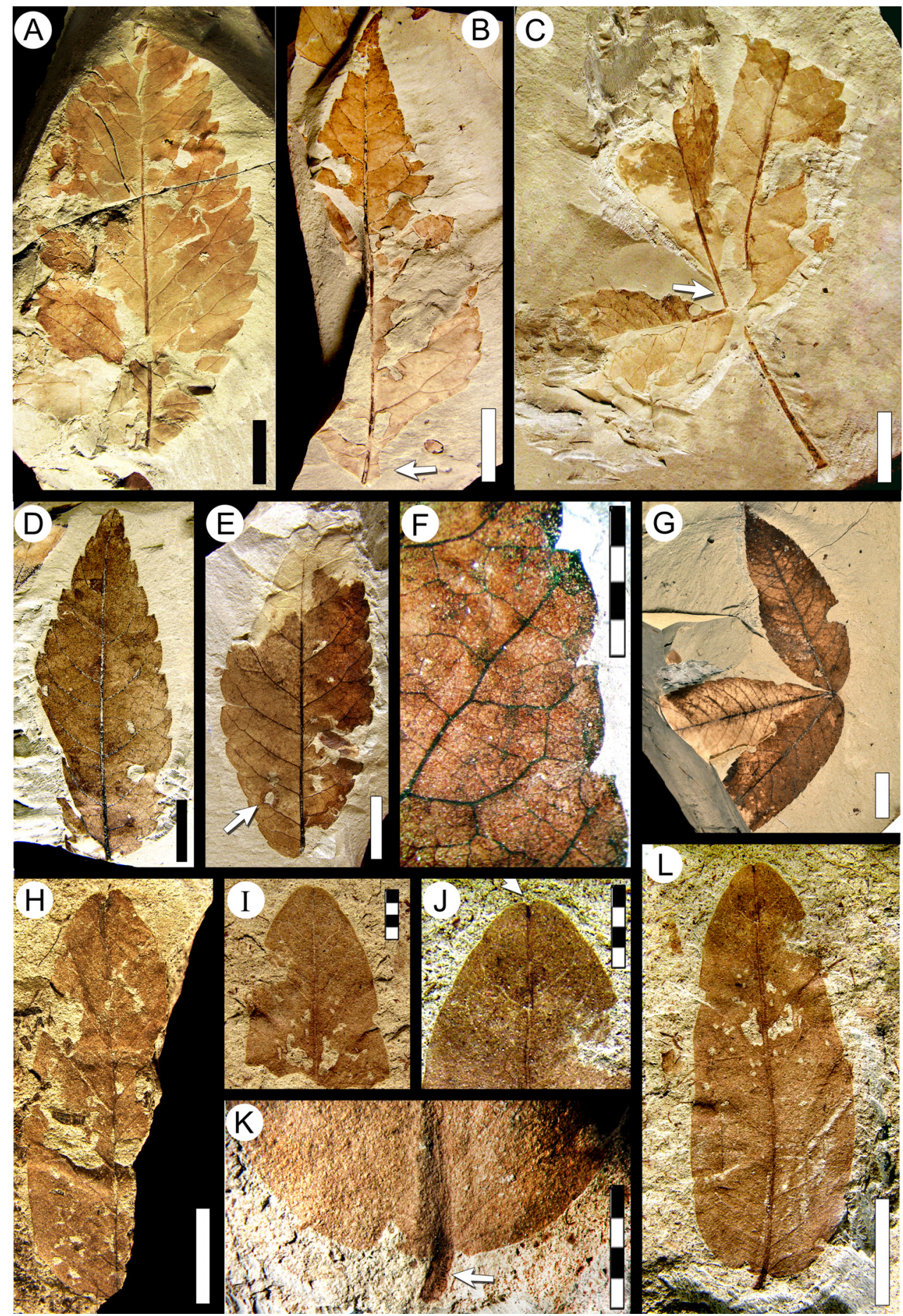

FIGURE 24. Morphotype SA043 (Cunoniaceae?/Sapindaceae? see also Figure 25E-F) and morphotype SA045 (Fabaceae; see also Figure 25J). A-G, morphotype SA043 (Cunoniaceae?/Sapindaceae?). A, MPEF-Pb-2036 (exemplar); B, MPEF-Pb-3023 (accessory exemplar 1; arrow, asymmetric base); C, articulated compound leaf, MPEF-Pb9110 (accessory exemplar 2; arrow, petiolule subtending terminal leaflet); D, MPEF-Pb-4102; E, MPEF-Pb-6561, with hole-feeding damage (DT113; see also Labandeira et al., 2007 p.7, figure DT113; and Donovan et al., 2016, figure 1i); F, tooth venation detail from Figure 24E; G, MPEF-Pb-9159 with three attached leaflets. H-L, morphotype SA045 (Fabaceae). H, MPEF-Pb-4110; I-L, MPEF-Pb-2038 (exemplar, see also Brea et al., 2008 figure 6, and Figure 25J); I, counterpart preserving apical portion; J, detail of retuse apex; (arrow); K, base detail with a pulvinulate, horizontally striated petiolule (arrow); L, complete exemplar. Single-color scale bars equal $10 \mathrm{~mm}$; grid scales equal one millimeter (per rectangle). 


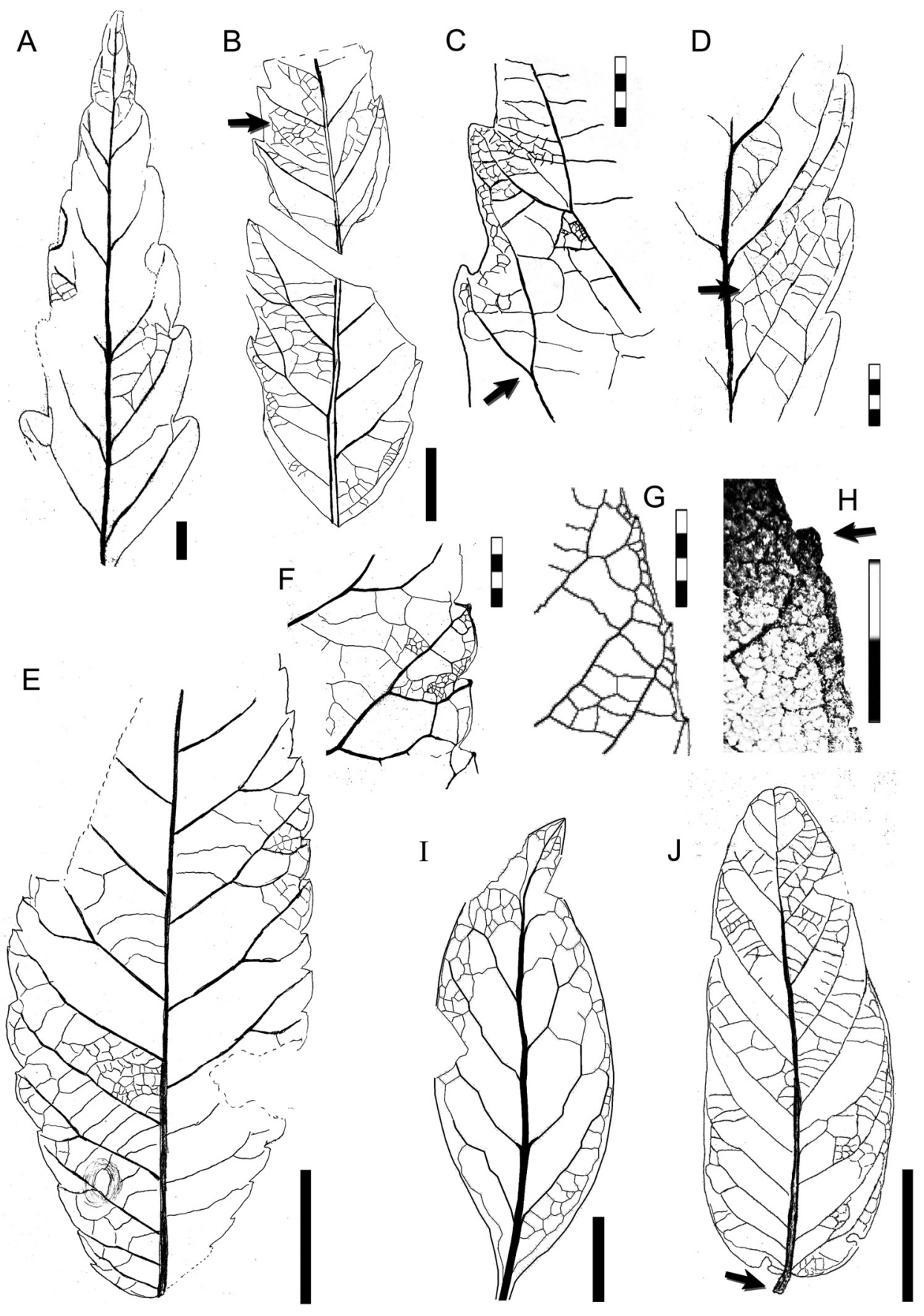

FIGURE 25. Morphotypes SA042 (Cunoniaceae; continued, see also Figure 16F-M), SA043 (Cunoniaceae?/Sapindaceae? continued, see also Figure 24A-G), SA044 (Cunoniaceae, see also Figure 23J-O), SA045 (Fabaceae; continued, see also Figure 24H-L), and SA046 Laurophyllum chubutensis Berry (Lauraceae, see also Figure 26A-F). A-D, camera lucida drawings (CLD) of morphotype SA042 (Cunoniaceae). A, MPEF-Pb-9069; B, MPEF-Pb-9113 (arrow, an intersecondary vein); C, tooth venation detail of specimen in Figure 25B (arrow, a forked secondary vein); D, tooth venation of specimen in Figure 25A (arrow, an intersecondary vein). E-F, morphotype SA043 (Cunoniaceae?/Sapindaceae?), CLD of MPEF-Pb-6561. G-H, morphotype SA044 (Cunoniaceae), MPEF-Pb-2037 (exemplar, see also Figure 23K, 23O). G, digital overlay drawing (DOD) of tooth venation; $\mathrm{H}$, triangular tooth with glandular apex (arrow). I, morphotype SA046 Laurophyllum chubutensis Berry (Lauraceae), DOD of MPEF-Pb-2039 (exemplar, see also Figure 26A-B), note low rank venation (see also Figure 26A-B). J, morphotype SA045 (Fabaceae), CLD of MPEF-Pb-2038 (exemplar; see also Figure 24I-L), note pulvinulate petiolule (arrow). Single-color scale bars equal $10 \mathrm{~mm}$; grid scales equal one millimeter (per rectangle). 


\section{Morphotype SA044}

Affinity. Cunoniaceae Brown, 1814 nom. cons. Exemplar. MPEF-Pb-2037 from quarry PL1 (Figures 23K, 23O, 25G-H).

Accessory exemplar. MPEF-Pb-3024 from quarry PL1 (Figure 23L).

\section{Figures 23J-O, 25G-H}

Description. Blade asymmetrical in base shape and/or insertion (Figure 23N, 23K). Size microphyll to notophyll, generally microphyll. Mean length 8.0 $(5.0-10.5) \mathrm{cm}$, mean width $2.0(1.1-4.0) \mathrm{cm}$. Mean length:width ratio 2.9:1 (2.2:1-5.6:1). Margin crenate or dentate (Figures 230, 25G-H). Petiole insertion marginal. Shape variable, generally wide elliptic or narrow elliptic. Apex shape straight, angle acute. Base shape decurrent (Figure 23N) or convex (Figure 23L), angle acute. Primary venation pinnate; primary vein thick, course slightly curved (Figure 23K). Basal veins one. Secondary venation craspedodromous, secondary veins numerous, opposite to alternate, thickness moderate, course regularly curved (Figure 23K-M), generally not branching but may fork near the blade base, running to the sinuses (Figure 25G). Isolated brochidodromous looping can develop in the largest leaves (Figure 23M). Secondary vein spacing increasing toward middle of blade (Figure 23M); vein angle smoothly increasing toward base (Figure 23L-M). Departure from midvein excurrent. Agrophic and intersecondary veins absent. Perimarginal venation a thin, irregular intramarginal vein (Figure 230). Intercostal tertiary venation thin, mixed opposite-alternate percurrent, departure from secondaries perpendicular (Figures 230, 25G-H). Epimedial tertiary venation opposite percurrent, thin, course sinuous, departure angle from primary perpendicular with uniform spacing. Fourth, fifth and sixth order venation regular polygonal reticulate (Figure 230). Freely ending veinlets not observed. Vein density $12.72 \mathrm{~mm} / \mathrm{mm}^{2}$ (one leaf measured). Areolation moderately developed. Marginal ultimate venation looped, forming a thin, irregular intramarginal vein (Figures 230, 25G). Teeth simple and very small (length $1.0-0.5 \mathrm{~mm}$ ), markedly asymmetrical; apex glandular (Figure $25 \mathrm{G}$ ), sometimes with a triangular capsule associated with the principal vein (Figures $230,25 \mathrm{H}$ ). Tooth shape concave/flexuous, straight/convex, straight/flexuous, or straight/concave; sinuses angular or rounded with extended concavity between teeth (Figure 25G). Tooth spacing uniform, two or three teeth per centimeter. Tooth venation supplied from a secondary running to the sinus, branching far from the tooth base and emitting a branch that innervates the tooth medially and another that runs near the basal flank of the supradjacent tooth (Figure 25G-H); tooth accessory venation looped, runing near the basal flank (Figure $25 \mathrm{H}$ ).

Observations. Distinguishing characters of this morphotype include: secondary veins numerous; teeth small, asymmetrical, and glandular; and principal tooth venation running to the sinus (see key in supplementary Appendix 2). Morphotype SA044 is present through all three Danian time intervals considered here and is abundant at the PL1 site (Table 1). The asymmetric base and the substantial variation in leaf architecture among leaves of different sizes suggests that morphotype SA044 may represent leaflets of a compound leaf. The teeth correspond to cunonioid teeth sensu Hickey and Wolfe (1975). The compound leaf, cunonioid teeth, and general leaf architecture resemble Australasian taxa in Cunoniaceae (e.g., Schizomeria spp., Pullea stutzeri [Mueller] Gibbs 1917, Aistopetalum viticoides Schlechter, 1915), and abundant Schizomerieae flowers occur at the same fossil localities (Jud et al., 2018a; see Introduction).

\section{Morphotype SA045}

Affinity. Fabaceae Lindley, 1836.

Exemplar. MPEF-Pb-2038 from quarry PL1 (Figures 24I-L, 25J).

Figures $24 \mathrm{H}-\mathrm{L}, 25 \mathrm{~J}$

Description. Blade symmetrical or weakly asymmetrical at the base, size microphyll. Length to 4.5 $\mathrm{cm}$, width to $2.0 \mathrm{~cm}$. Length:width ratio $\sim 2: 1$ (one leaf measured). Margin entire. Petiolule (presumed) short, pulvinulate with horizontal striations, insertion marginal (Figures 24K, 25J). Shape slightly ovate. Apex shape convex or retuse (Figures $24 \mathrm{H}, 24 \mathrm{~L}, 25 \mathrm{~J}$ ), angle acute. Base shape rounded (Figure 24K), angle acute. Basal veins three, two of them very thin and running near the margin (Figure 25J). Primary venation pinnate; midvein thick with course slightly curved, reaching the apical margin with strength (Figure 24H, 24L). Secondary venation brochidodromous; in seven 11 pairs of thin veins, course uniformly curved; spacing smoothly decreasing toward the base, angle increasing toward base. Secondaries decurrent on midvein (Figure 25J). Perimarginal venation absent. Intercostal tertiary venation mixed opposite-alternate percurrent, departing perpendicularly from secondaries. Epimedial tertiary venation mixed opposite-alternate percurrent, course straight; angle decreasing toward base (Figure 
25J). Freely ending veinlets not observed. Marginal ultimate venation looped.

Observations. Morphotype SA045 can be placed in the Fabaceae Lindley, 1836 based on its pulvinulate, horizontally striated petiolule, weakbrochidodromous secondary venation with thin secondaries running parallel to the margin for some distance, and retuse apex. This leaflet type was previously described along with co-occurring, permineralized Fabaceae wood (mimosoid clade) from the same unit (Brea et al., 2008, figure 6) as the oldest reliable record of legumes from the Southern Hemisphere. This occurrence is approximately coeval with the Danian fossil legumes recently reported from Corral Bluffs in Colorado (Lyson et al., 2019). Morphotype SA045 is only known from five total specimens at quarries OR2 and PL1 (Salamanca Formation, Table 1).

\section{Morphotype SA046}

Equivalent to. Laurophyllum chubutensis Berry, 1937, syntype USNM-208530 (Berry, 1937a, plate IX, figure 3; here Figure 26F, Table 2).

Affinity. Lauraceae Jussieu, 1789.

Exemplar. MPEF-Pb-2039 from quarry PL1 (Figures 25I, 26A-B).

Accessory exemplar. MPEF-Pb-3025 from quarry PL1 (Figure 26E).

Figures 25I, 26A-F

Description. Blade symmetrical, size microphyll to notophyll, generally microphyll. Mean length 6.1 (3.7-9.5) cm, mean width (2.9-4.4) cm. Mean length:width ratio 3.9:1 (2.5:1-6.7:1). Margin entire. Petiole robust, base swollen, insertion marginal. Shape elliptic (Figure 26A, 26E) or obovate (Figure 26C). Apex shape straight extended (Figure 26C$\mathrm{F}$ ), angle acute. Base shape decurrent on the petiole (Figure 25I), angle acute. Primary venation pinnate. Midvein thick, course straight or somewhat curved. Basal veins one. Agrophic veins absent. Secondary venation reticulodromous (Figures 25I, 26A) or slightly brochidodromous (Figure 26C). Secondary vein course irregular, branching (Figure 26B) a thin pair of acute basal secondaries present (Figure 25I), otherwise spacing and angle uniform. Perimarginal venation a thin fimbrial vein (Figure 26B). Intersecondary veins proximally parallel to subjacent secondary (Figure 26C); length less than half that of subjacent secondary; distally reticulating or ramifying; one or fewer per secondary. Intercostal tertiary venation random reticulate to irregular percurrent (Figure 26B). Epimedial tertiary venation mixed opposite percurrent and reticulate, angle variable with respect to primary, obtuse toward base, decreasing exmedially. Fourth and fifth order veins random reticulate. Freely ending veinlets not observed. Vein density $9.12 \mathrm{~mm} / \mathrm{mm}^{2}$ (one leaf measured). Areolation well developed (Figure 26B). Marginal ultimate venation joining the fimbrial vein. Leaf rank $2 r$.

Observations. Morphotype SA046 can be placed in the family Lauraceae Jussieu, 1789 based on its entire margin, fimbrial vein, thick cuticle (that conforms to Lauraceae; under separate study), pinnate primary venation with a thick midvein, acute basal secondary veins, thin secondary venation, and well-developed third to fifth orders of venation. Morphotype SA046 differs from other entire-margined, pinnate-veined leaf morphotypes studied here (i.e., morphotypes SA010, SA056, SA063, SA073, SA077, and SA078) in that it has secondaries with lower angles, lacks agrophic veins, and has a conspicuous fimbrial vein and a lower foliar rank (see key in supplementary Appendix 2). Morphotype SA046 is equivalent to one syntype specimen of Laurophyllum chubutensis Berry, 1937a (USNM-208530 only, Figure 26F) described from the Palacio de los Loros locality (Table 2). Even though Berry's original description lacks detail, the type material shares all leaf characters with morphotype SA046. This morphotype occurs at most quarries from the Salamanca Formation (Table 1).

\section{Morphotype SA047}

Affinity. Unknown.

Exemplar. MPEF-Pb-2040 from quarry PL2 (Figures 26G, 26K, 27A).

Figures 26G-L, 27A

Description. Blade symmetrical, size nanophyll to mesophyll, generally notophyll, mean length 6.5 $(4.6-8.5) \mathrm{cm}$, mean width $4.3(1.4-7.0) \mathrm{cm}$. Mean length:width ratio 1.8:1 (1.3:1-3.2:1). Margin serrate (Figure 26I-L). Petiole insertion marginal. Shape elliptic, palmately 3-lobed, lobes triangular (Figure 26G-H, 26L), lobe apex acuminate and spinose (Figure 26L), lobe sinuses rounded. Apex shape straight or concave (Figure 26I). Base shape decurrent, angle acute (Figures 26G-H, 27A). Primary venation basal actinodromous with three primaries of straight course (Figure 27A). Basal veins three. Agrophic veins simple (Figures 26G-H, 27A), may bifurcate near the margin, more commonly on smaller leaves. Secondary venation craspedodromous. Secondaries few, thick, subopposite. Secondary vein spacing $1.5-2.0 \mathrm{~cm}$, increasing toward the base, arise at uniform angles, course slightly curved. Secondaries branching near margin, main branch entering the 


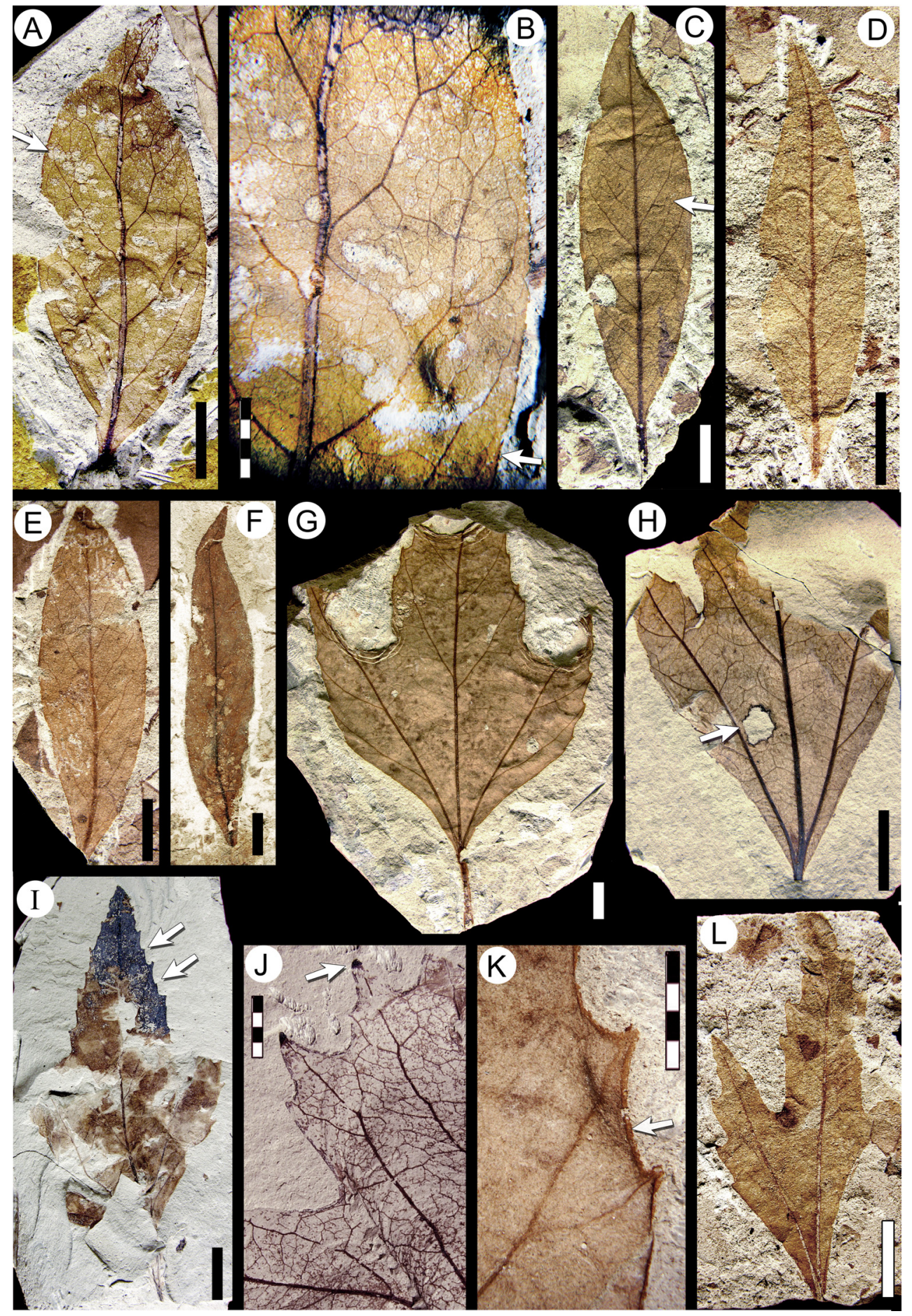

FIGURE 26. Morphotype SA046 Laurophyllum chubutensis Berry (Lauraceae; continued, see also Figure 25I) and morphotype SA047 (see also Figure 27A). A-F, morphotype SA046 (Lauraceae). A, MPEF-Pb-2039 (exemplar, see also Figure 25I), note patches of skeletonization (arrow, DT16; see also Donovan et a., 2018 figure 6.1); B, venation detail of specimen in Figure 26A (exemplar; arrow, fimbrial vein); C, MPEF-Pb-9114 (arrow, intersecondary vein); D, MPEF-Pb-4115; E, MPEF-Pb-3025 (accessory exemplar); F, Laurophyllum chubutensis Berry, 1937a syntype, USNM208530 (illustrated in Berry, 1937a, plate IX, figure 3-4; see also Tables 1, 2). G-L, morphotype SA047. G, MPEF-Pb2040 (exemplar, see also Figure 27A); H, MPEF-Pb-9116, with hole feeding damage (DT03, arrow; see also Donovan et al., 2016); I, MPEF-Pb-4124 (arrow, second order teeth); J, detail of compound teeth, MPEF-Pb-9117 (arrow indicating glandular tooth apex); K, tooth and venation detail of specimen in Figure 26G (exemplar), note the fimbrial vein (arrow); L, MPEF-Pb-4121. Single-color scale bars equal $10 \mathrm{~mm}$; grid scales equal one millimeter (per rectangle). 

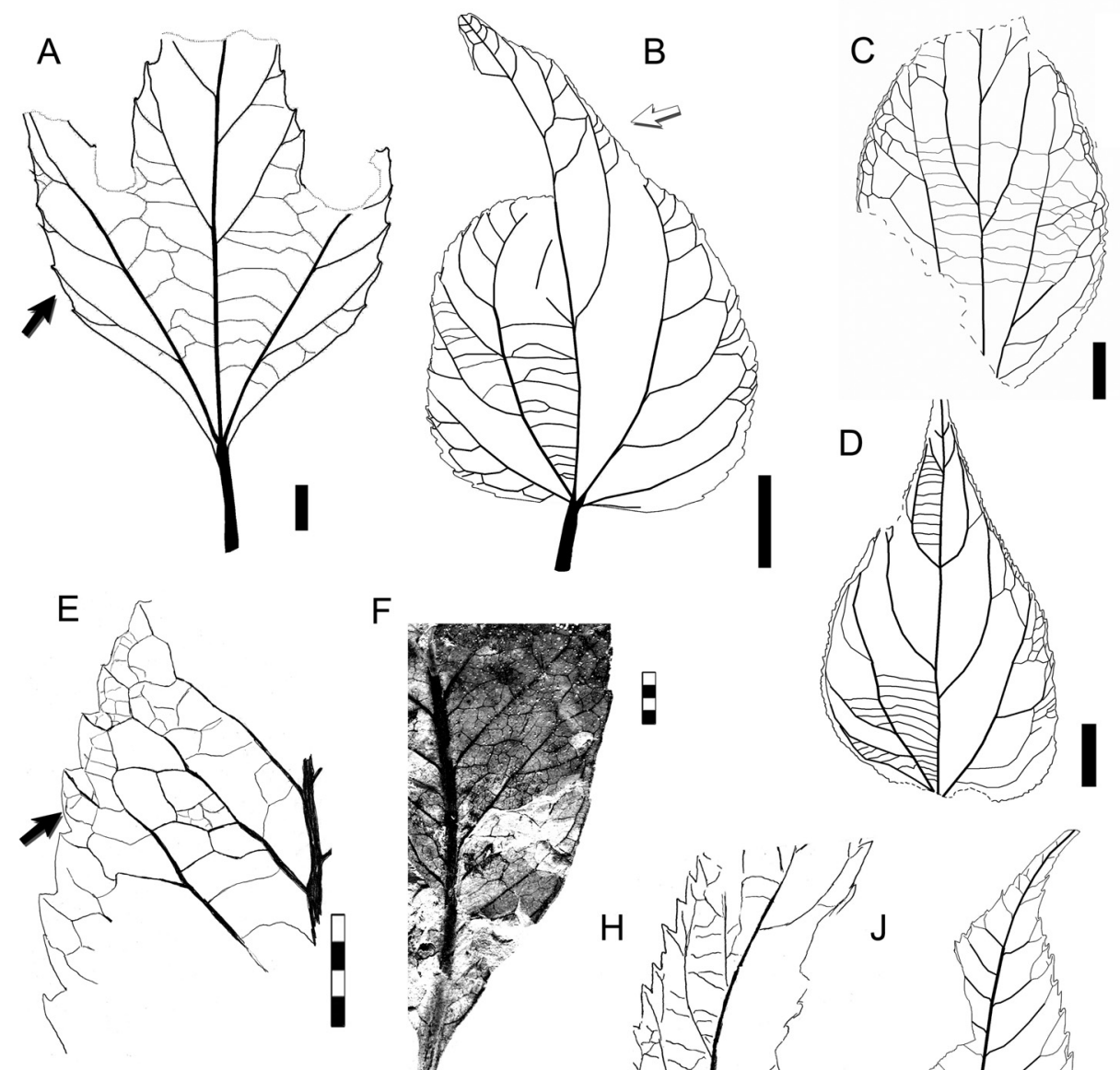

$\mathrm{F}$
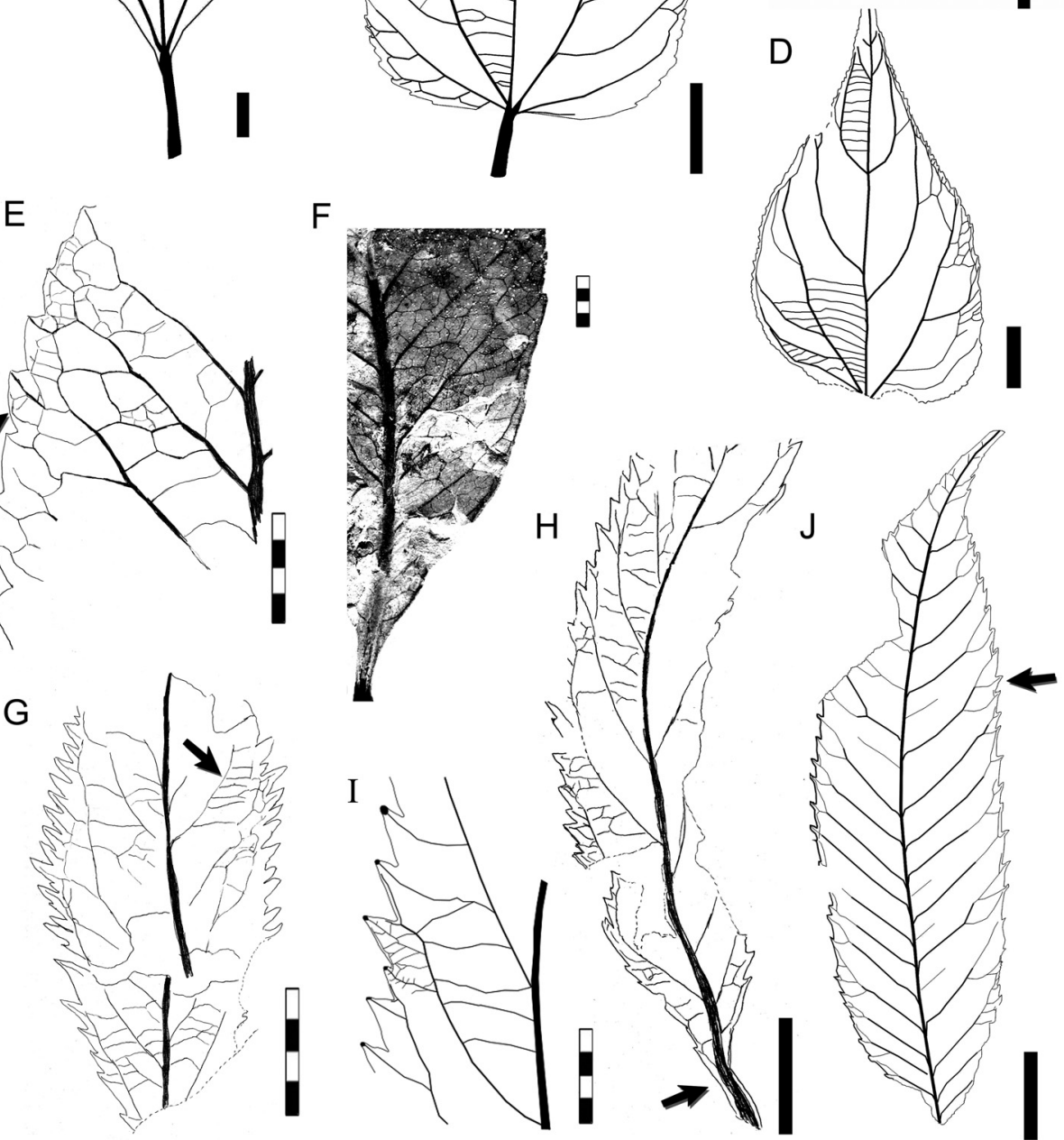

FIGURE 27. Morphotypes SA047 (continued, see also Figure 26G-L), SA048 (Rhamnaceae, see also Figure 28A-G), SA049 "Myrica" premira Berry 1937a (see also Figure 29A-K), SA051 (Urticaceae? see also Figure 30A-G), and SA052 (Sapindaceae? see also Figure 28H-I). A, morphotype SA047, digital overlay drawing (DOD) of MPEF-Pb2040 (exemplar; arrow, agrophic veins; see also Figure 26G, 26K). B-D, morphotype SA048 (Rhamanceae). B, DOD of MPEF-Pb-2041 (exemplar, see also Figure 28D), note compound agrophic veins (arrow); C, DOD of Ziziphus chubutensis Berry, 1937a holotype USNM-208529 (see also Figure 28B and Table 2); D, DOD of "Banaraphyllum" ovatum Berry, 1937a holotype USNM-201953 (see also Figure 28A and Table 2). E-F, morphotype SA049 "Myrica" premira. E, DOD of tooth venation of MPEF-PB-2042 (exemplar; see also Figure 29D) (arrow, an independent tooth accessory vein); F, venation and margin of MPEF-Pb-9118. G-I, morphotype SA051 (Urticaceae?). G, camera lucida drawing (CLD) of MPEF-Pb-3028 (accessory exemplar; see also Figure 30C, 30E) (arrow, percurrent tertiaries); $\mathrm{H}$, CLD of MPEF-Pb-2044 (exemplar; see also Figure 30A-B) (arrow, decurrent leaf base). I, DOD of teeth detail from Figure 27H (exemplar). J, morphotype SA052 (Sapindaceae?), DOD of MPEF-Pb-2045 (exemplar, see also Figure $28 \mathrm{H}-\mathrm{I}$ ); note secondary vein branches (arrow). Single-color scale bars equal $10 \mathrm{~mm}$; grid scales equal one millimeter (per rectangle). 
tooth (Figure 26J-K). Secondaries decurrent on midvein (Figure 27A). Perimarginal venation a thick fimbrial vein (Figure 26K). Intersecondary and interior secondary veins absent. Intercostal tertiary venation mixed opposite-alternate percurrent, departing perpendicularly from secondaries (Figure 26J). Epimedial tertiary venation opposite percurrent, tertiaries disposed perpendicular to primaries, course convex, angles inconsistent (Figures $26 \mathrm{H}$, $27 \mathrm{~A})$. Fourth and fifth order venation regular polygonal reticulate (Figure 26J). Freely ending veinlets not observed. Marginal ultimate venation joining the fimbrial vein and associated to small veins (Figure 26J-K). Teeth simple (Figure 26G-H) or compound in two distinct orders (Figure 26I-J); asymmetrical, glandular, and recurved (Figure 26J). Tooth shape concave/convex or concave/ flexuous; sinuses rounded Tooth spacing uniform; one tooth per centimeter (Figure 27A). Tooth venation supplied by secondaries or secondary basal branches forming the principal veins, deflected by tertiary junctions (Figure 26J); an apical branch run to the sinus and innervates both sinus flanks; or other accessory veins, including the fimbrial vein and other minor veins running inside the tooth margin. Tooth apex with an opaque gland.

Observations. Morphotype SA047 is present through all three Danian time intervals considered here (Table 1). It is easily recognized from its 3lobed form, prominent fimbrial vein, and its glandular teeth (Figure 26J; supplementary Appendix 2).

\section{Morphotype SA048}

Equivalent to. Ziziphus chubutensis Berry, 1937 holotype USNM-208529 (Berry, 1937a, plate IX, figure 2; here Figures $28 \mathrm{~B}$ and redrawn in $27 \mathrm{C}$ ); and "Banaraphyllum" ovatum Berry, 1937 holotype USNM-201953 (Berry, 1937a, plate IX, figure 1; here Figure 28A and redrawn in Figure 27D) (Table 2).

Affinity. Rhamnaceae Jussieu, 1789.

Exemplar. MPEF-Pb-2041 from quarry PL1 (Figures 27B, 28D-E).

\section{Figures 27B-D, 28A-G}

Description. Blade asymmetrical with asymmetrical base (Figure 28D, 28F), size nanophyll to mesophyll, generally notophyll. Mean length 6.2 $(2.1-10.5) \mathrm{cm}$, mean width $(1.2-7.1) \mathrm{cm}$. Mean length:width ratio 1.6:1 (1:1-2:1). Margin crenate (Figure 28E-F). Petiole stout, length $2-3 \mathrm{~cm}$, insertion marginal (Figures 27B, 28D, 28F). Shape wide ovate. Apex shape straight or slightly acuminate, angle acute. Base shape rounded, truncate, or concavo-convex; angle obtuse (Figure 28C-D,
28F). Primary venation basal actinodromous (Figure 27B). Basal veins five, one thicker primary, two sets of thinner lateral primaries, at least one primary naked at base (Figures 27B, 28C-D). Secondary venation eucamptodromous. Secondary veins sparse, only 3-4 pairs, course uniformly curved; spacing increasing toward base; secondaries arise decurrent on midvein at uniform angles (Figure 27B-D). Major and minor secondary veins exmedially branch and join in loops (semicraspedodromous). Agrophic veins compound with branching minor secondary veins (Figure 27B). Perimarginal and intersecondary veins absent. Intercostal tertiary venation opposite percurrent, departing from secondaries perpendicularly (Figure $28 \mathrm{E})$. Epimedial tertiary venation opposite percurrent, thin, course convex or sinuous, densely spaced, departing from midvein perpendicularly; angle slightly increasing exmedially (Figure 27BD). Fourth order venation indistinct. Areolation and freely ending veinlets not observed. Marginal ultimate venation of incomplete loops (Figure 28E). Teeth simple, asymmetric; tooth apex sometimes with a small gland (Figure 28E). Tooth shape convex/convex or straight/convex. Sinuses rounded. Tooth spacing uniform, five to seven per centimeter and four to five per secondary vein (Figure 28E-F). Tooth venation supplied by a curving secondary vein branch that runs near the apical flank and reaches the tooth apex; the principal vein can generate branches that innervate the tooth flank (Figure $28 \mathrm{E}$ ). Sinuses irrigated with a separated, straight secondary branch.

Observations. Morphotype SA048 is present through all three Danian time intervals considered here and is abundant at the PL1 site (Table 1). Its characters are identical to those of the type specimens of both "Banaraphyllum" ovatum Berry, 1937 (USNM-201953, Figures 27D, 28A) and Ziziphus chubutensis Berry, 1937 (USNM-208529, Figures 27C, 28B), which were described from well preserved but incomplete specimens collected at the Palacio de los Loros locality. Berry (1937a) placed these specimens in different genera and families despite the lack of marked differences between them. Based on a larger collection, both type specimens may be placed in the range of morphological variation we define for morphotype SA048 (Table 2). The specimens closely resemble extant Rhamnaceae, especially Ziziphus Miller, 1754 species as first described (Berry 1937a). As mentioned earlier, Jud et al. (2017) described fossil flowers and leaves related to the ziziphoid/ampelozizyphoid clades from the Rancho Grande (RG) locality 


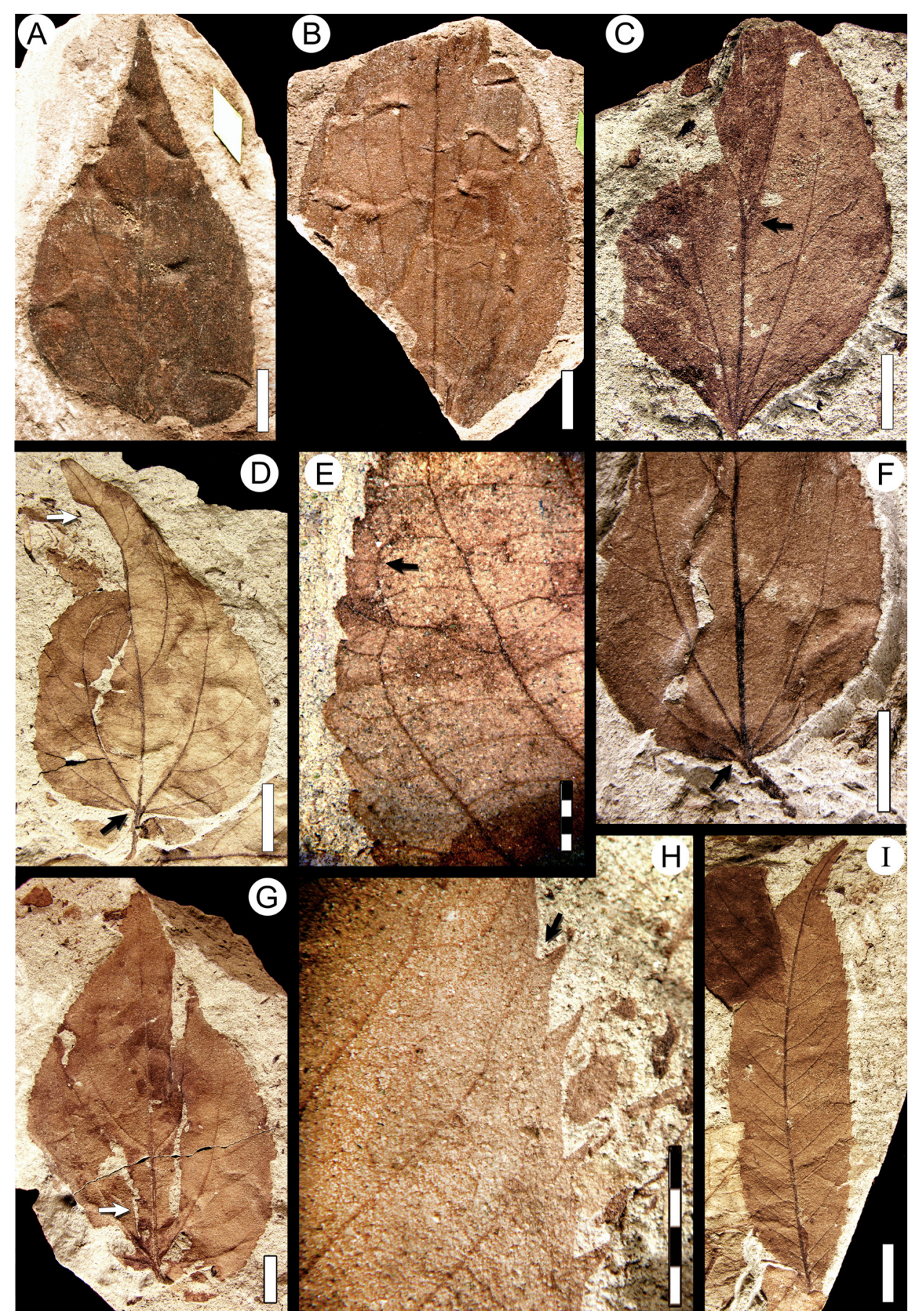

FIGURE 28. Morphotype SA048 (Rhamnaceae; continued, see also Figure 27B-D) and morphotype SA052 (Sapindaceae? continued, see also Figure 27J). A-G, morphotype SA048 (Rhamnaceae). A, "Banaraphyllum" ovatum Berry, 1937a holotype, USNM-201953 (illustrated in Berry, 1937a, plate IX, figure 1; see also Figure 27D and Tables 1, 2); B, Ziziphus chubutensis Berry, 1937a holotype, USNM-208529 (illustrated in Berry, 1937a, plate IX, figure 2; see also Figure 27C and Tables 1, 2); note the Cochlichnus trace fossils; C, MPEF-Pb-4129 (arrow, decurrent secondary veins); D, MPEF-Pb-2041 (exemplar, see also Figure 27B), note the naked basal vein (black arrow) and the margin feeding leaf damage (DT14, white arrow; see also Donovan et al., 2016); E, tooth detail from specimen in Figure 28D (arrow, an independent sinus vein); F, leaf base of MPEF-Pb-9119, note the naked basal vein (black arrow); G, MPEF$\mathrm{Pb}-9120$, with hole feeding leaf damage (DT05, arrow; see also Donovan et al., 2016). H-I, morphotype SA052 (Sapindaceae?), MPEF-Pb-2045 (exemplar, see also figure 27J). H, detail of the teeth and higher order venation (arrow, tooth venation forking at the sinus); I, complete (probable) leaflet. Single-color scale bars equal $10 \mathrm{~mm}$; grid scales equal one millimeter (per rectangle). 
(Chron C29n, Salamanca Formation, Figure 1); however, those leaves are quite different from morphotype SA048. Distinguishing characters of this morphotype include: blade ovate and asymmetrical; primary veins basal actinodromous; secondary veins eucamptodromous; five basal veins including at least one naked basal primary; and small, asymmetrical teeth (see key in supplementary Appendix 2 ). The most similar rhamnaceous morphotype in this study, morphotype SA069, is basally symmetrical and acrodromous with a crenulate margin and prominent, chevroned interior secondary veins (Figure 34J).

\section{Morphotype SA049}

Equivalent to. "Myrica" premira Berry, 1937 in part: syntype USNM-201963 (Berry, 1937a, plate $\mathrm{V}$, figure 4; here Figure 29A-B) only (Table 2), syntype USNM-201962 excluded (see morphotype SA020).

Affinity. unknown.

Exemplar. MPEF-PB-2042 from quarry PL2 (Figures 27E, 29D).

Accessory exemplars. MPEF-Pb-3026 from quarry PL2 (Figure 29F-G), and MPEF-Pb-3027 from quarry PL1 (Figure 29E).

Figures 27E-F, 29A-B, 29D-K

Description. Blade symmetric or slightly asymmetric (Figure 29A, 29I-J). Size nanophyll to notophyll, generally microphyll. Mean length 6.3 (2.4-12.0) $\mathrm{cm}$, mean width $2.2(0.7-4.5) \mathrm{cm}$. Length:width ratio variable, generally narrow (less than 2:1). Margin generally crenate with teeth more developed toward apex (Figure 29I-K), to nearly entire (Figure 29F-G) with only very small, irregularly spaced crenations. Petiole strong, slightly curved, insertion marginal, base swollen (Figure 29D). Shape very variable, generally obovate (Figure 29D, 29I-K) or elliptic (Figure 29E-F). Apex shape convex (Figure 29l-J), straight (Figure 29E), or rounded (Figure 29K); angle acute. Base shape decurrent, angle acute (Figure 29D). Primary venation pinnate; primary vein wide, course straight, or slightly curved; reaching the leaf apex without branching. Basal veins one. Secondary venation semicraspedodromous or festooned-semicraspedodromous (Figure 29E-G), sometimes reticulodromous near the apex (Figure 29K). Secondary veins numerous and thin, subopposite, course irregular, occasionally forking. Spacing and angle to midvein very variable, irregular. Perimarginal and agrophic veins absent. Intersecondary veins almost match secondaries in gauge, proximally and distally parallel to subjacent secondary, longer than half the length of subjacent secondary, frequency one or less per secondary (Figure 29G, 29K). Intercostal tertiary venation random reticulate (Figures 27E-F, 29G-H). Epimedial tertiary venation percurrent or random reticulate; tertiary angle inconsistent; course mixed (concave, convex, or sinuous). Fourth order venation random polygonal reticulate. Fifth order venation dichotomizing. Freely ending veinlets with dendritic branching (Figure $29 \mathrm{H}$ ). Vein density range $13.45-15.17 \mathrm{~mm} / \mathrm{mm}^{2}$. Areolation poorly developed. Marginal ultimate venation looped (Figure 29G). Foliar rank 2r. Teeth simple, generally small to cryptic (Figure 29G), reaching 2 $\mathrm{mm}$ in length (Figures 27E, 29K). Tooth shape convex/convex, straight/convex, flexuous/convex, or flexuous/flexuous; sinuses angular Tooth spacing uniform, up to four teeth per centimeter. Tooth venation supplied by a secondary vein or branch, reaching the supradjacent sinus or running near the tooth apical flank (Figure 27E). An accessory independent vein running near the basal flank of the tooth can occur.

Observations. Morphotype SA049 is present through all three Danian time intervals considered here and is abundant at the PL2 quarry (Table 1). It has a broad and continuous range of variation in many leaf architectural characters but is clearly distinguishable from other leaf morphotypes based on: blade obovate or elliptic; base decurrent; secondary veins thin and numerous, irregular in course and spacing, semicraspedodromous or festooned semicraspedodromous; low foliar rank; and teeth simple (see key in supplementary Appendix 2). Also, piercing and sucking insect-damage (e.g., DT46 of Labandeira et al., 2007; Figure 29F) occurs frequently on this leaf morphotype (Donovan et al., 2018).

The obovate leaf shape, decurrent base shape, extended base angle, irregularly coursing secondary veins, low foliar rank, poorly developed teeth, and coriaceous texture of morphotype SA049 are identical to the type specimen of "Myrica" premira Berry, 1937a (USNM-201963, Figure 29A-B, Table 2), described based on specimens from the Palacio de los Loros locality. The other type specimen USNM-201962 (Figure 29C), differs from morphotype SA049, and with Berry's (1937a) written description for " $M$." premira, and is placed here with morphotype SA020 (Table 2).

"Myrica" premira was originally compared to "M." mira Berry, 1925a from the Eocene Laguna del Hunco flora, northwest Patagonia, but we find no similarities between them, nor of either species with Myricaceae. Eocene M. mira has an ovate to 


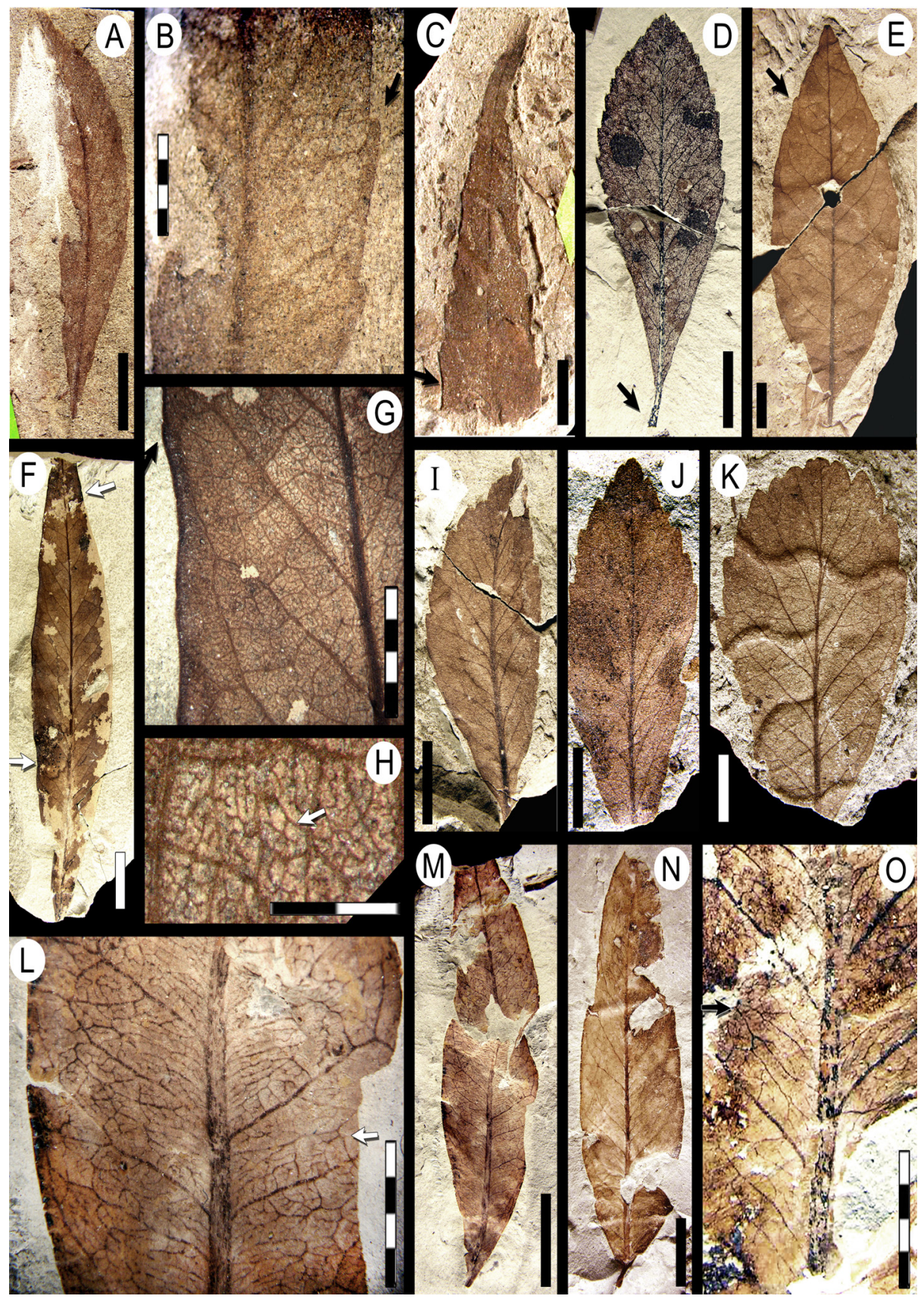

FIGURE 29. Morphotype SA049 "Myrica" premira Berry (continued, see also Figure 27E-F), morphotype SA050 (Anacardiaceae? see also Figure 31A-D), and related material. A-K, morphotype SA049 "Myrica" premira (except Figure 29C). A, "M." premira syntype USNM-201963 (illustrated in Berry, 1937a, plate V, figure 4; see also Table 2); B, tooth detail (arrow) from specimen in Figure 29A; C, "M. premira" syntype USNM-201962 (illustrated in Berry, 1937a, plate V, figure 3; here referred to morphotype SA020 Cunoniaceae; see also Tables 1, 2), note the thick fimbrial vein (arrow); D, MPEF-Pb-2042 (exemplar, see also Figure 27E), note swollen petiole base (arrow); E, MPEF-Pb-3027 (accessory exemplar 1) (arrow, a crenation); F, MPEF-Pb-3026 (accessory exemplar 2), with piercing and sucking leaf damage (DT 46, arrows; see also Donovan et al., 2016), often found on this leaf morphotype; G, venation detail from specimen in Figure 29F (arrow, a crene); $\mathrm{H}$, higher order venation of specimen in Figure 29F, note dendritic branching of freely ending veinlets (arrow); I, MPEF-Pb-4135; J, MPEF-Pb-4134, with piercing and sucking leaf damage (DT 46; see also Donovan et al., 2016); K, MPEF-Pb-4136, with Cochlichnus trace fossils. L-O, morphotype SA050 (Anacardiaceae?). L, higher-order venation from Figure 29M, note admedially ramified tertiary venation (arrow); M, MPEF-Pb2043 (exemplar, see also Figure 31A); N, MPEF-Pb-4143; O, leaf venation detail of MPEF-Pb-9150, showing admedially ramified venation (arrow). Single-color scale bars equal $10 \mathrm{~mm}$; grid scales equal one millimeter (per rectangle). 
elliptic shape, craspedodromous venation with straight secondary course, and a serrate margin with well-developed teeth deployed in distinctive pairs, each pair associated with a single secondary.

\section{Morphotype SA050}

Affinity. Anacardiaceae? Brown, 1814.

Exemplar. MPEF-Pb-2043 from quarry PL2 (Figures 29L-M, 31A).

Figures 29L-O, 31A-D

Description. Blade symmetrical; size microphyll to notophyll, generally microphyll. Mean length 7.0 $(2.4-12.5) \mathrm{cm}$, mean width $2.3(0.7-2.5) \mathrm{cm}$. Length:width ratio $\sim 3: 1$. Margin entire, irregularly wavy (undulating, Figures 29N, 31A). Petiole insertion marginal. Shape narrow ovate. Apex shape convex, angle acute. Base shape cuneate, angle acute. Primary venation pinnate; primary vein thick, course straight, reaching the apex with strength. Basal veins three, one medial primary and two intramarginal veins (Figure 31A, 31C). Agrophic veins absent. Secondary venation reticulodromous (Figure 31A-D), secondary veins thin and numerous, course irregular, branching near the margin one to three times, occasionally forming irregular loops. Spacing and angles irregular. Perimarginal venation an intramarginal vein, well developed at the leaf base (Figure 31C). Intersecondary veins thick, proximally and distally subparallel to subjacent secondaries, longer than half the length of the subjacent secondary (Figure 29L), frequency one or fewer per secondary. Intercostal tertiary venation admedially ramified, tertiaries tending to be disposed parallel to secondaries (Figures 29L, 290, 31B-C), generating compound intersecondaries. Epimedial tertiary venation reticulate (Figures 29L, 31B). Fourth order venation dichotomizing (Figure 31D). Freely ending veinlets one-branched. Vein density $8.40 \mathrm{~mm} / \mathrm{mm}^{2}$ (one leaf measured). Areolation poorly developed. Marginal ultimate venation looped Figure 31B. Foliar rank $3 r$.

Observations. Morphotype SA050 was found at low abundance at quarries OR1 and PL2 (Table 1). The characteristic irregular, admedially ramified tertiary venation and the presence of an intramarginal vein exhibited in morphotype SA050 are commonly found among the Anacardicaceae (e.g., Sorindeia Thouars, 1806, Dracontomelon Blume, 1851, Micronychia Oliver, 1881) and to some degree in Myrtaceae (e.g., Myrceugenia Berg, 1854 and Eugenia Linnaeus, 1753), which is repre- sented by wood from the Salamanca Formation, Myrceugenia chubutense Ragonese 1980.

\section{Morphotype SA051}

Affinity. Urticaceae? Jussieu, 1789 nom. cons.

Exemplar. MPEF-Pb-2044 from quarry PL2 (Figures 27H-I, 30A-B, 30G).

Accessory exemplar. MPEF-Pb-3028 from quarry PL2 (Figures 27G, 30C, 30E-F).

Figures 27G-I, 30A-G

Description. Blade symmetric, size nanophyll to microphyll, generally microphyll. Mean length to $5.3(3.8-6.8) \mathrm{cm}$, mean width to $1.48(1.2-2.3) \mathrm{cm}$. Length:width ratio $\sim 5.5: 1$. Margin serrate. Petiole thick, up to $1 \mathrm{~cm}$ in length, insertion marginal (Figure 30G). Shape elliptic or narrow ovate. Apex shape straight, angle acute (Figure 30A). Base shape decurrent, angle acute (Figures 27H, 30G). Primary venation pinnate; midvein thickness moderate, course curved to irregular. Basal veins three. Secondary venation semicraspedodromous, tending to craspedodromous; secondary veins subopposite on the midvein, moderate in thickness, course uniformly curved and running parallel to margin distally, joining to supradjacent secondary with several tertiary veins, giving off basal tertiaries that innervate teeth (Figures $27 \mathrm{H}-\mathrm{I}, 30 \mathrm{E}$ ). Spacing and angle of secondaries to midvein uniform (Figure 30A). Secondaries decurrent on midvein (Figure $27 \mathrm{H}$ ). Perimarginal venation a fimbrial vein (Figure 30B). Agrophic and intersecondary veins absent. Intercostal tertiary venation opposite percurrent, departing from secondaries perpendicularly (Figure $27 \mathrm{H}-\mathrm{I})$. Epimedial tertiary venation opposite percurrent, disposed perpendicular to primary, course convex, spacing uniform (Figure $27 \mathrm{G}-\mathrm{H}$ ). Fourth and fifth order venation regular polygonal reticulate. Fifth order venation very thin. Freely ending veinlets not observed. Areolation well developed. Marginal ultimate venation is the fimbrial vein. Teeth well developed, simple or compound, may have a minor cusp on basal flank (Figures 27I, 30B). Tooth shape irregular and asymmetrical, generally triangular (Figure 30C), apex spinose or spherulate. Tooth apex with a very thick spherule, sometimes preserved with some relief (Figures 27I, 30B-C, 30E), up to $0.1 \mathrm{~mm}$ in diameter, standing out from the tooth apex. Tooth shape straight/flexuous, straight/convex, or straight/concave; sinuses angular. Tooth spacing uniform, dense, five to eight teeth per centimeter (Figures 27G-H, 30F). Tooth venation supplied by one medial vein from a secondary or a tertiary vein; 


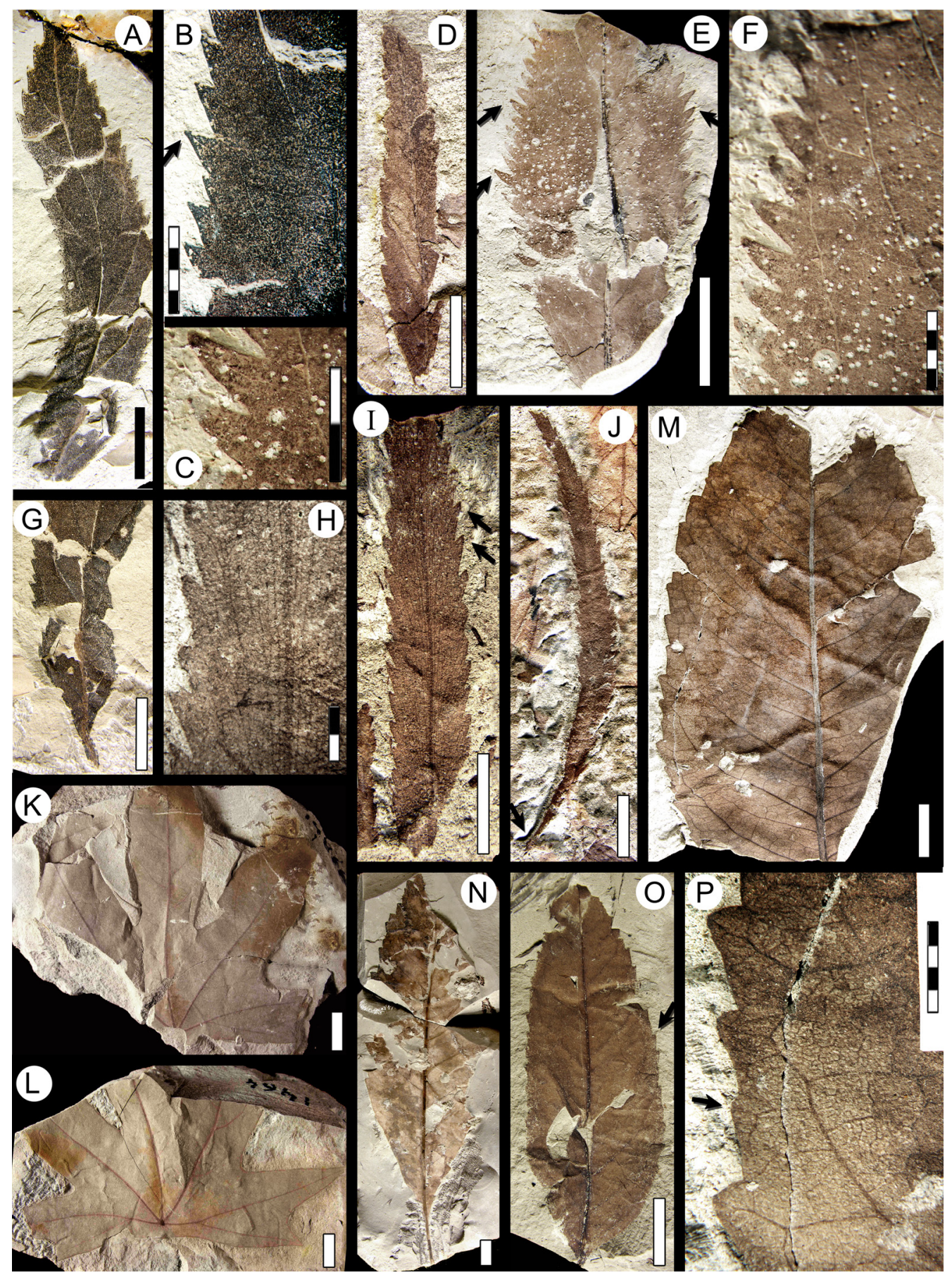

FIGURE 30. Morphotypes SA051 (Urticaceae? continued, see also Figure 27G-I), SA053 (see also Figure 31E-F), SA054 (Cunoniaceae; see also Figure 31G-H), and SA055 (continued, see also Figure 21E-F). A-G, morphotype SA051 (Urticaceae?). A, MPEF-Pb-2044 (exemplar, see also Figure 27H-I); B, tooth detail from specimen in Figure 30A (exemplar; arrow, spherulate apex); C, tooth detail from specimen in Figure 30E (accessory exemplar); D, MPEF$\mathrm{Pb}-4145$; E, MPEF-Pb-3028 (accessory exemplar; see also Figure 27G; arrows, spherulate teeth); F, venation detail from specimen in Figure 30E (accessory exemplar); G, leaf base from counterpart of specimen in Figure 30A. H-J, morphotype SA053. H, parallel venation detail from Figure 30I (exemplar); I, MPEF-Pb-3029 (exemplar; see also Figure 31E) (arrows, glandular teeth); J, MPEF-Pb-9121 (arrow, swollen petiole base; see also Figure 31F). K-L, morphotype SA055 MPEF-Pb-2047, part and counterpart (exemplar, see also Figure 21E-F). M-P, morphotype SA054 (Cunoniaceae). M, MPEF-Pb-2046 (exemplar, see also Figure 31G-H); N, MPEF-Pb-9122; O, MPEF-Pb-4147 (arrow, a glandular tooth apex); $\mathrm{P}$, tooth venation detail from Figure 30M (exemplar; arrow, a second-order tooth). Single-color scale bars equal $10 \mathrm{~mm}$; grid scales equal one millimeter (per rectangle). 


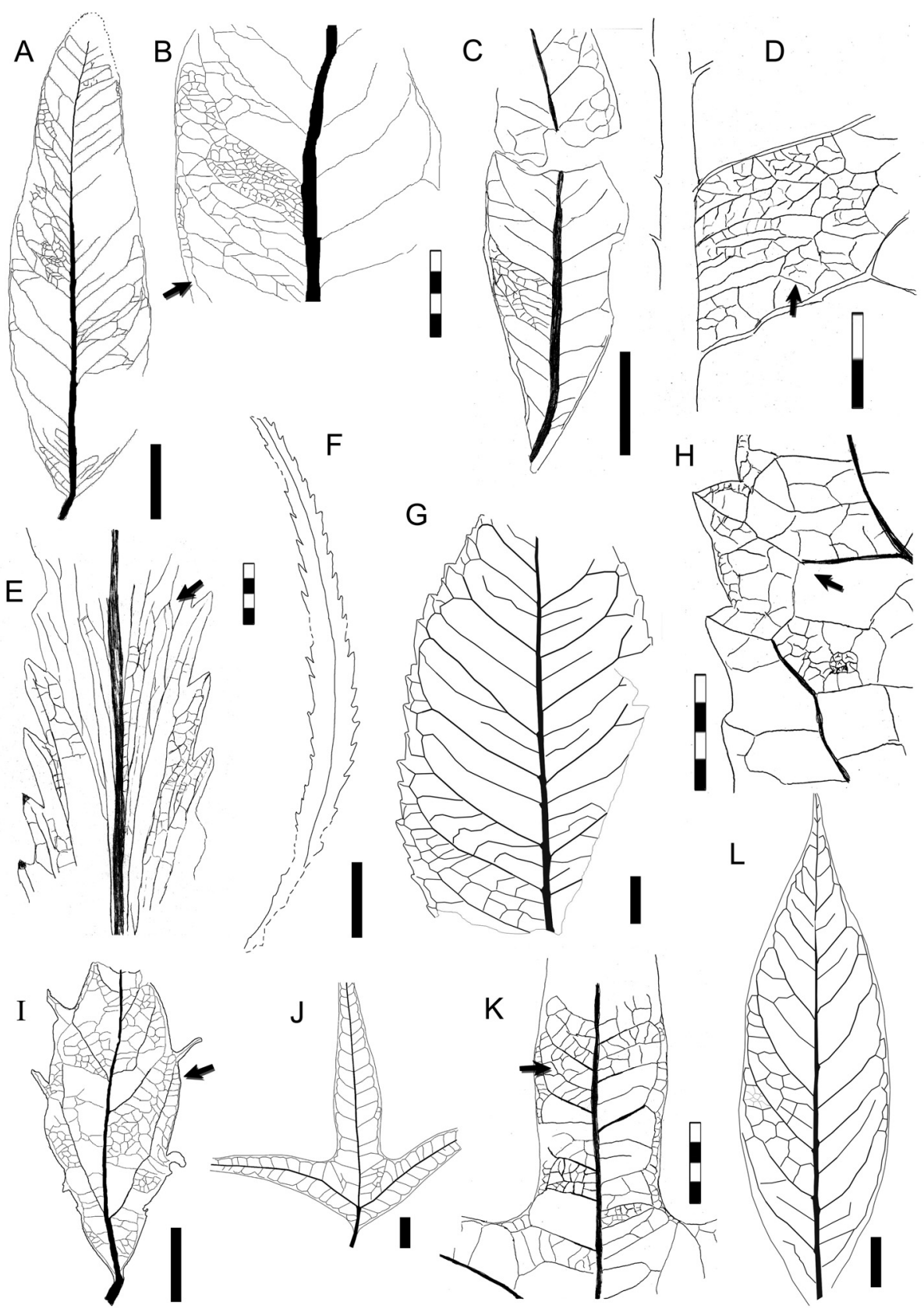

FIGURE 31. Morphotypes SA050 (Anacardiaceae? continued, see also Figure 29L-O), SA053 (continued, see also Figure 30H-J) SA054 (continued, see also Figure 30M-P), SA056 (see also Figure 32A-C), SA057 (see also Figure 32D-G), and SA058 (Malvaceae? see also Figure 32H-J). A-D, morphotype SA050 (Anacardiaceae?). A, digital overlay drawing (DOD) of MPEF-Pb-2043 (exemplar, see also Figure 29L-M); B, higher order venation detail from Figure 30A (arrow, intramarginal vein); C, camera lucida drawing (CLD) of MPEF-Pb-9123; D, higher order venation detail from Figure 31C (arrow, a dichotomizing fourth-order vein). E-F, morphotype SA053. E, CLD of MPEF-Pb-3029 (exemplar, see also Figure 30H-l; arrow, a secondary vein anastomosis); F, DOD of MPEF-Pb-9121 (from Figure 30J). G-H, morphotype SA054, MPEF-Pb-2046 (exemplar, see also Figure 30M, 30P). G, DOD of complete leaf; H, CLD of tooth venation, note vein junction (arrow) before entering the tooth. I, morphotype SA057, DOD of MPEF-Pb-2049 (exemplar, see also Figure 32D-G), note proximal secondary veins extended for over half the length of the blade (arrow). J-K, morphotype SA058 (Malvaceae?). J, DOD of MPEF-Pb-2050 (exemplar, see also Figure 32H, 32J); K, CLD of higher order venation from Figure 30J (exemplar; arrow, intersecondary vein). L, morphotype SA056, DOD of MPEF-Pb-2048 (exemplar, see also Figure 32A, 32C). Single-color scale bars equal $10 \mathrm{~mm}$; grid scales equal one millimeter (per rectangle). 
two thin accessory veins arise from other tertiary veins that fork near the sinus (Figure 27I).

Observations. Morphotype SA051 is easily recognized from its narrow blade; numerous, prominent compound teeth; and robust secondary veins. The teeth of morphotype SA051 are the salicoid type (Hickey and Wolfe, 1975), having a medial vein ending in a callose gland and two or more thinner accessory veins attenuating toward the apex. Morphotype SA051 resembles several Urtica spp. (e.g., U. dioica Linnaeus, 1753). Morphotype SA051 has been found only at quarries PL1 and PL2 (Table 1), at low abundance.

\section{Morphotype SA052}

Affinity. Sapindaceae? Jussieu, 1789 nom. cons. Exemplar. MPEF-PB-2045 from quarry PL1 (Figures $27 \mathrm{~J}, 28 \mathrm{H}-\mathrm{I})$.

Figures $27 \mathrm{~J}, 28 \mathrm{H}-\mathrm{I}$

Description. Blade and base asymmetrical, size microphyll. Length $9.0 \mathrm{~cm}$, width $2.2 \mathrm{~cm}$. Length:width ratio $\sim 4: 1$. Margin serrate. Petiolule (presumed) insertion marginal (Figure 28I). Shape narrow oblong. Apex shape acuminate extending into 'drip tip' (Figure 28I), angle acute. Base shape concave, angle acute (Figures 27J, 28I). Primary venation pinnate; primary vein thick, course uniformly curved. Basal veins one. Secondary venation craspedodromous, secondaries thin and dense, subopposite on midvein, course abruptly curved near the margin, may branch once or more before reaching the margin (Figure 27J), generating thin branches that innervate the teeth medially. Secondary vein spacing and angle somewhat irregular, smoothly decreasing toward base. Departure from midvein excurrent. Perimarginal, agrophic, and intersecondary veins absent. Intercostal tertiary venation alternate percurrent, angle obtuse at departure from secondaries (Figure $28 \mathrm{H})$. Epimedial tertiary venation alternate percurrent, course straight, tertiary angles obtuse to primary and uniform. Freely ending veinlets not observed. Areolation well developed. Marginal ultimate venation looped. Teeth simple, well developed, asymmetrical (Figure $28 \mathrm{H}$ ). Tooth shape straight/flexuous, straight/convex, or flexuous/convex; sinuses angular. Tooth spacing irregular, four per centimeter, one to three teeth per secondary vein. Tooth venation supplied by one principal vein, deflected apically by a tertiary vein directed to the sinus, then forking and entering the tooth along the distal flank and reaching the apex (Figure 28I).

Observations. Morphotype SA052 has an asymmetrical oblong blade and a curved drip tip sugges- tive of origin from a compound leaf, numerous irregular secondaries and irregular teeth, and thick veins entering the tooth sinuses, characters that are typical of leaflets in Sapindaceae and other families in Sapindales Jusseau ex Berchtold and Presl, 1820. Distinctive characters of this morphotype include: blade oblong; apex acuminate; secondary veins craspedodromous, subopposite, with branches to the tooth sinuses; teeth simple; and tooth venation directed from the sinus, then entering the tooth along the distal flank (see key in supplementary Appendix 2). Morphotype SA052 has only been found at the PL1 quarry, at low abundance (Table 1).

\section{Morphotype SA053}

Affinity. Unknown.

Exemplar. MPEF-Pb-3029 from quarry PL1 (Figures $30 \mathrm{H}-\mathrm{I}, 31 \mathrm{E}$ ).

Figures $30 \mathrm{H}-\mathrm{J}$, 31E-F

Description. Blade medially and basally asymmetrical. Size microphyll. Maximum length $8.0 \mathrm{~cm}$, mean width to $0.7(0.6-0.9) \mathrm{cm}$. Length:width ratio $>7: 1$. Margin serrate (Figure 30I-J). Petiole insertion marginal, petiole short and swollen (Figure 30J). Shape elliptic to oblong, very narrow. Texture chartaceous. Apex shape straight, angle narrow acute (Figure 31F). Base shape decurrent, angle acute (Figure 30J). Primary venation composed of a multistranded midvein $0.7 \mathrm{~mm}$ wide (Figure $30 \mathrm{H}$ ). This primary vein cluster acts as one basal vein. Agrophic veins absent. Secondary venation cladodromous, very thin and dense, arising decurrently from the midvein at a low angle (Figures $30 \mathrm{H}, 31 \mathrm{E})$, spacing irregular, angle decreasing toward base. Secondary course straight, subparallel, rarely anastomosing (Figures $30 \mathrm{H}, 31 \mathrm{E}$ ), basally branched, entering teeth or forming wellmarked sinus-bracing veins (Figure 31E). Perimarginal vein a fimbrial vein. Higher-order veins of indistinct orders, opposite percurrent with straight course (Figure 31E), sometimes resembling thin, compound intersecondary veins. Freely ending veinlets not observed. Vein density $6.12 \mathrm{~mm} / \mathrm{mm}^{2}$ (one leaf measured). Marginal ultimate venation joining the fimbrial vein. Teeth well developed, simple, triangular, oriented at acute angles respect to midvein, with glandular apices (Figures $30 \mathrm{H}, 31 \mathrm{E}$ ). Tooth shape straight/flexuous or straight/convex; sinuses angular. Tooth spacing uniform, two-three per centimeter (Figure 31F). Tooth venation supplied by secondary veins running medially; two accessory veins running along both tooth flanks that reach the midvein before reaching the tooth 
apex (Figure 31E); three accessory veins (two in the tooth basal flank) sometimes joining the principal vein; and numerous additional tertiary veins.

Observations. Distinguishing characters of this morphotype include: blade very narrow; midvein multistranded; secondary venation notably dense, thin, low-angled, and cladodromous, and the teeth numerous and triangular (see key in supplementary Appendix 2). Morphotype SA053 has been found at low abundance at several quarries in the Salamanca Formation (Table 1). The venation and development of the teeth correspond to the chloranthoid tooth type, in which the apex shape is generally acuminate and the venation is supplied by a medial vein and two lateral veins that reach the medial vein at or before the apex (Hickey and Wolfe, 1975). The multistranded medial vein giving off numerous subparallel, forking lateral veins at low angles and the tooth venation and density resemble some Apiaceae Lindley, 1836 (e.g., Eryngium Linnaeus, 1753).

\section{Morphotype SA054}

Affinity. Cunoniaceae? Brown, 1814 nom. cons. Exemplar. MPEF-PB-2046 from quarry PL2 (Figures 30M, 30P, 31G-H).

\section{Figures 30M-P, 31G-H}

Description. Blade symmetrical, base asymmetrical (Figure 300), size microphyll to mesophyll, generally notophyll. Mean length $9.8(7.0-12) \mathrm{cm}$, mean width $4.0(2.2-6.0) \mathrm{cm}$. Mean length:width ratio 2.7:1 (2.2:1-3.2:1). Margin dentate. Shape ovate (Figure 300). Apex shape convex, angle acute. Base shape convex (Figure 300) or decurrent (Figure $30 \mathrm{~N}$ ), angle acute. Primary venation pinnate; primary vein thick (slightly thick at secondary vein departures, Figure $31 \mathrm{G}$ ), course slightly curved. Basal veins three; two thin lateral veins and the medial vein. Agrophic veins absent. Secondary venation semicraspedodromous; secondaries thin, opposite to subopposite, course slightly curved, branching exmedially and forming a series of loops (Figures 30M, 31G). Secondary vein spacing slightly increasing toward base. Departure from midvein excurrent, at wide angles, angle increasing toward base. Perimarginal venation a fimbrial vein, thicker at leaf base (Figure $30 \mathrm{~N}$ ). Intersecondary veins proximally parallel to subjacent secondary, to slightly obtuse, longer than half the length of subjacent secondary, distally reticulating or ramifying; frequency less than one per secondary (Figures $30 \mathrm{M}, 31 \mathrm{G}$ ). Intercostal tertiary venation regular reticulate, dense. Epimedial tertiary venation reticulate to percurrent, perpendicular to primary, course convex, angle decreasing exmedially (Figure $31 \mathrm{G}$ ). Fourth and fifth order venation regular polygonal reticulate (Figure $31 \mathrm{H})$. Freely ending veinlets branch twice or more (Figure $31 \mathrm{H}$ ). Vein density $5.43 \mathrm{~mm} / \mathrm{mm}^{2}$ (one leaf measured). Areolation moderately developed. Marginal ultimate venation looped (Figure $31 \mathrm{H}$ ). Teeth triangular, compound with a minor tooth on the basal flank of the major tooth (Figures 30P, 31G), apex glandular with a small, dark, triangular cap. Tooth shape concave/straight, convex/convex or convex/straight; sinuses angular. Tooth spacing uniform, two to three teeth per centimeter (Figure 30M). Tooth venation supplied by a medial vein from the acute junction of two secondary veins or from a tertiary vein arising from a secondary loop, accessory veins looped (Figures 30P, 31H).

Observations. Morphotype SA054 has been found only at the PL2 quarry (Table 1), at low abundance. This morphotype has several characters shared among Cunoniaceae leaves (e.g., Weinmannia affinis Gray, 1854). Characters that distinguish this morphotype from similar cunonioid leaflets in the present work (i.e., SA005, SA020, SA042, SA043, SA44, SA049; Table 1) include: blade broad ovate; intersecondary and firmbrial veins present; teeth triangular and compound; and tooth venation supplied by a medial vein from a junction of two veins.

\section{Morphotype SA055}

Affinity. Unknown.

Exemplar. MPEF-Pb-2047 from quarry PL2.

Figures 21E-F, 30K-L

Description. Blade symmetrical, size mesophyll. Length $7.9 \mathrm{~cm}$, width $11.42 \mathrm{~cm}$ (from one specimen). Length:width ratio $\sim 0.7: 1$. Shape palmately lobed, ovate in outline. Texture chartaceous. Margin entire. Petiole insertion peltate eccentric (Figure $30 \mathrm{~L}$ ), incerted $0.6 \mathrm{~cm}$ from basalmost margin. Shape palmately 7-lobed, ovate in outline, lobe shape ovate to elliptic (Figure 30K). Apex angle obtuse, including all lobes (Figure 21E-F). Base shape cordate, angle wide-obtuse (Figure 30L). Lobe apex angle acute, shape straight (Figure $30 \mathrm{~L}$ ). Primary venation basal actinodromous with five primary veins arising from the eccentric petiolar insertion (Figure 21E); primary course slightly sinuous, the outermost pair forked once, innervating small basal lobes (Figure 21E). Basal veins five. Secondary venation eucamptodromous (Figure $21 \mathrm{~F}$ ); secondary veins ending at intramarginal vein. Secondaries somewhat variable, may be alternate to subopposite; course uniformly curved; 
running parallel to margin. Secondary spacing increasing toward base; secondary angle acute and decreasing toward base. Secondaries decurrent on midvein. Perimarginal venation an intramarginal vein developed around the whole margin (Figure 21E). Interior secondaries convex, chevroned (Figure 21E). Sinus-bracing veins thin, not forking. Agrophic and intersecondary veins absent. Intercostal tertiary venation mixed opposite-alternate percurrent departing from secondaries perpendicularly (Figure 21F). Epimedial tertiary venation mixed percurrent, course straight, tertiary angles perpendicular to primaries, angle uniform across whole blade. Fourth order veins regular polygonal reticulate (Figure 21F). Freely ending veinlets not observed. Areolation well developed. Marginal ultimate venation joining the intramarginal vein.

Observations. Morphotype SA055 is known from only two specimens, from quarries PL2 (Salamanca Formation) and LF (Peñas Coloradas Formation) (Table 1), and it has a unique leaf architecture: no other fossil leaves in the flora share its eccentrically peltate, palmately largelobed form.

\section{Morphotype SA056}

Affinity. Unknown.

Exemplar. MPEF-PB-2048 from quarry PL1 (Figures 31L, 32A, 32C).

\section{Figures 31L, 32A-C}

Description. Blade symmetrical, size microphyll to notophyll, generally microphyll. Mean length 9.5 $(7.5-12.0) \mathrm{cm}$, mean width $3.1(2.5-3.4) \mathrm{cm}$. Mean length:width ratio $3: 1$ (2.5:1-3.4:1). Margin entire. Petiole insertion marginal. Shape elliptic. Apex shape acuminate forming a short 'drip tip', angle acute (Figure 32A-B). Base shape straight, angle acute. Primary venation pinnate; primary vein thick, course straight (Figure 32A). Basal veins three, formed by one medial and two thin laterals. Secondary venation brochidodromous; secondary veins decurrent on midvein, subopposite to alternate, course uniformly curved to abruptly curved near the margin; losing gauge exmedially and becoming as thin as tertiaries, joining supradjacent vein by one or a series of arches, sometimes generating second order loops. Secondary veins arise at acute angles from primary, angle and spacing uniform throughout the blade. Perimarginal venation a fimbrial vein. Intersecondary veins compound, parallel to secondary (Figure 32C), less than one per secondary. Agrophic veins absent. Intercostal tertiary venation random reticulate (Fig- ure 32C). Epimedial tertiary venation alternate percurrent, course straight, perpendicular to primary, angles increasing toward base (Figure 31L). Fourth order veins random polygonal reticulate. Fifth order veins admedially dichotomizing, sometimes reticulate. Vein density $8.56 \mathrm{~mm} / \mathrm{mm}^{2}$ (one leaf measured). Areolation moderately developed. Freely ending veinlets one- or two-branched. Marginal ultimate venation joining the fimbrial vein.

Observations. Morphotype SA056 differs from other, similar entire-margined pinnate-veined leaf morphotypes (i.e., SA010, SA046, SA063, SA073, SA077, and SA078) in having elliptic shape, notophyll size, no agrophic veins, weak-brochidodromous secondary venation, high foliar rank (almost $3 r$ ), and chartaceous texture. Morphotype SA056 is present through all three Danian time intervals considered here, at low abundance (Table 1).

\section{Morphotype SA057}

Affinity. Unknown. Exemplar. MPEF-Pb-2049 from quarry PL2.

Figures 31I, 32D-G

Description. Blade symmetrical, size microphyll, length $4.6 \mathrm{~cm}$, width $1.7 \mathrm{~cm}$. Length:width ratio 2.7:1 (one leaf measured). Margin dentate. Petiole length $9.0 \mathrm{~mm}$, width $1.3 \mathrm{~mm}$; insertion marginal. Shape elliptic (Figure 32G). Apex shape not preserved, angle acute. Base shape convex, angle acute (Figure 31I). Primary venation pinnate; primary vein thick, course slightly curved. Basal veins three, one primary and two secondaries that become fimbrial veins (Figure 31I). Agrophic veins simple, looped. Secondary venation semicraspedodromous; secondary veins thick, alternate, opposite at base, decurrent on midvein; secondary angles acute, irregular, and decreasing toward base; spacing increasing toward the base. Proximal secondaries extend for over half the length of the blade parallel to margin (Figure 31I), course irregular, entering teeth near the margin. Perimarginal venation a thick fimbrial vein (Figure 32F). Intersecondary veins absent. Intercostal tertiary, epimedial tertiary, fourth, and fifth order venation regular polygonal reticulate (Figures 31I, 32F). Freely ending veinlets unbranched, terminating in idioblasts (preserved as dark dots, Figure 32F) throughout the blade. Vein density $11.00 \mathrm{~mm} / \mathrm{mm}^{2}$ (one leaf measured). Areolation well developed, areole shape six-sided. Marginal ultimate venation joining the fimbrial vein. Teeth simple, long and narrow (Figure 32D-E), length $4.0-7.0 \mathrm{~mm}$, width $0.8 \mathrm{~mm}$, isolated. Tooth shape straight/convex or straight/straight, sometimes recurved, tooth base 


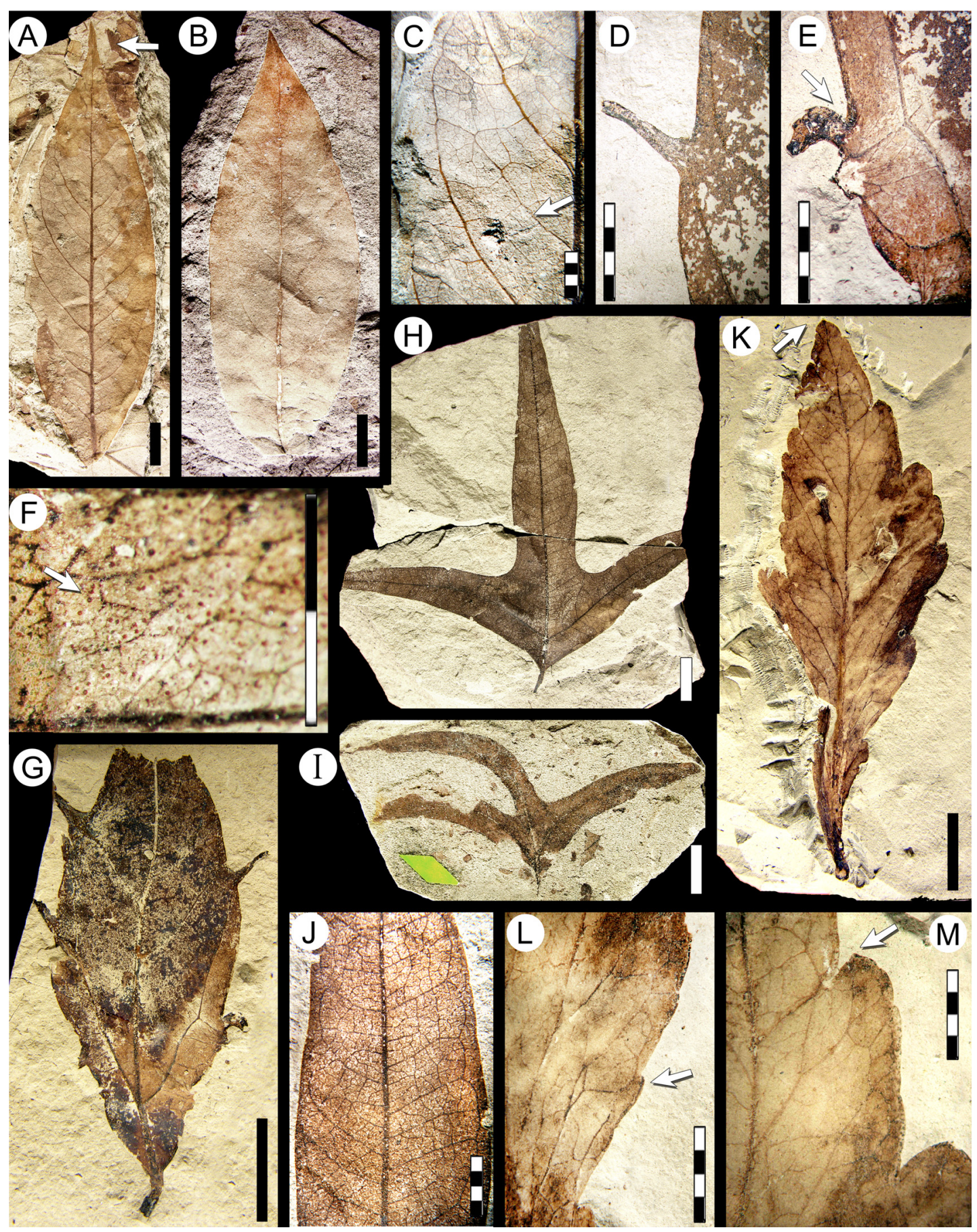

FIGURE 32. Morphotype SA056 (continued, see also Figure 31L), SA057 (continued, see also Figure 31I), SA058 (Malvaceae? continued, see also Figure 31J-K), and SA059 (see also Figure 33A-B). A-C, morphotype SA056. A, MPEF-Pb-2048 (exemplar; see also Figure 31L; arrow, drip tip); B, MPEF-Pb-4150; C, higher order venation from specimen in Figure 32A (exemplar; arrow, an intersecondary secondary vein). D-G, morphotype SA057, MPEF-Pb2049 (exemplar, see also Figure 31I). D, spinose tooth; E, tooth venation detail, with margin-feeding leaf damage (DT12, arrow; see also Donovan et al., 2016); F, higher order venation and idioblasts (dark dots, arrow); G, complete leaf of exemplar. H-J, morphotype SA058 (Malvaceae?). H, MPEF-Pb-2050 (exemplar, see also Figure 31J-K); I, "Sterculia" acuminataloba Berry, 1937a syntype USNM-208525 (illustrated in Berry 1937a, plate VIII, figure 2; see also Table 2); J, venation detail from specimen in Figure 32H (exemplar). K-M, morphotype SA059, MPEF-Pb-2051 (exemplar, see also Figure 33A-B). K, complete leaf of exemplar, note mucronate apex (arrow); L, basal teeth (arrow) and venation detail; $\mathrm{M}$, detail of apical compound teeth and venation (arrow, a small, opaque, spherulate tooth apex). Single-color scale bars equal $10 \mathrm{~mm}$; grid scales equal one millimeter (per rectangle). 
slightly expanded, apex acute; sinuses rounded (Figure 32G). Tooth spacing irregular, approximately one per $1.3 \mathrm{~cm}$. Tooth venation supplied by a single tertiary vein arising from a secondary loop or a secondary branch (Figure 31I).

Observations. Morphotype SA057 has distinctive non-glandular, narrow-elongate, widely spaced teeth; it is known from a single specimen from the PL2 quarry (Table 1).

\section{Morphotype SA058}

Equivalent to. "Sterculia acuminataloba" Berry, 1937, in part: syntype USNM-208525 (Berry, 1937a, plate VIII, figure 2; here Figure 32I) only (Table 2); syntypes USNM-208524, USNM208526, USNM-208527, and USNM-208528 excluded (see morphotypes SA014 and SA019).

Affinity. Malvaceae? Jussieu, 1789 nom. cons.

Exemplar. MPEF-Pb-2050 from quarry PL2 (Figures $31 \mathrm{~J}-\mathrm{K}, 32 \mathrm{H}, 32 \mathrm{~J}$ ).

Accessory exemplar. MPEF-Pb-3030 from quarry PL1.

Figures $31 \mathrm{~J}-\mathrm{K}, 32 \mathrm{H}-\mathrm{J}$

Description. Blade symmetrical, size microphyll to mesophyll, generally notophyll. Length to $8.9 \mathrm{~cm}$, width to $9.8 \mathrm{~cm}$. Length:width ratio $\sim 0.9: 1$. Margin entire. Petiole robust, more than $6.0 \mathrm{~mm}$ long and $1.1 \mathrm{~mm}$ wide; insertion marginal (Figures 31J, $32 \mathrm{H}$ ). Texture chartaceous. Shape palmately 3lobed, ovate in outline. Blade apex angle obtuse. Lobe apex straight, angle acute. Lobes thin and deeply incised (Figure 32H-I). Base shape straight or concave, angle obtuse. Primary venation suprabasal actinodromous with two lateral opposite or subopposite primaries; primary veins thick, course straight (Figure $32 \mathrm{H}-\mathrm{I}$ ). Basal veins three (Figure 31J). Secondary venation brochidodromous, secondaries alternate to subopposite, abruptly curved near the margin, joining supradjacent vein at nearright angles, tending to form a series of loops or a thin, sinuous intramarginal vein; departure from primary at wide, variable angles (Figure 32J). Secondaries dense and uniformly spaced. Perimarginal, agrophic, and sinus-bracing veins absent. Intersecondary veins proximally parallel to subjacent secondary; longer than half the length of subjacent secondary; distally reticulating or ramifying; one or more per secondary. Intercostal tertiary venation opposite percurrent, joining to secondaries perpendicularly, angle tending to increase exmedially. Epimedial tertiary venation opposite percurrent, course sinuous, perpendicular to primaries. Fourth and fifth order venation regular polygonal reticulate (Figure $31 \mathrm{~K}$ ). Freely ending veinlets not observed. Vein density $12.57 \mathrm{~mm} / \mathrm{mm}^{2}$ (one leaf measured). Areolation moderately developed. Marginal ultimate venation looped.

Observations. Morphotype SA058 is present through all three Danian time intervals considered here (Table 1), at low abundance. It shares all leaf characters with syntype USNM-208525 of "Sterculia" acuminataloba Berry, 1937 (Figure 32I), which shows very different leaf architecture from the other type specimens of "S." acuminataloba that we placed here in other morphotypes (i.e., SA014, SA019; see key in supplementary Appendix 2). There are many fossil leaf species from Patagonia that have been referred to Sterculia Linnaeus, 1753 that superficially resemble morphotype SA058, but none corresponds in the details (also see discussion for morphotypes SA014, SA019). Some specimens of Sterculia kurtzii Hünicken, 1995 (Hünicken 1995, plate C, figures 1-4, 6-21) from the Cretaceous flora of the Cerro Cazador Formation, southern Chile, are comparable to morphotype SA058 in having a trilobate form with suprabasal actinodromous primary venation, thin intramarginal veins, and well-developed lobes. However, S. kurtzii differs in having wider triangular lobes, straight to cuneate lobe apices, sub-rounded sinuses, and sinus-bracing veins. Regular actinodromous, lobed leaves are typical of but not exclusive to Malvaceae, including Sterculia and other genera (Carvalho et al., 2011).

\section{Morphotype SA059}

Affinity. Unknown.

Exemplar. MPEF-Pb-2051 from quarry PL2.

\section{Figures 32K-M, 33A-B}

Description. Blade symmetrical, size microphyll, length $6.8 \mathrm{~cm}$, width $2.5 \mathrm{~cm}$. Length:width ratio 2.7:1 (one leaf measured). Texture membranaceous. Margin dentate. Petiole short (length $4 \mathrm{~mm}$, width $2 \mathrm{~mm}$ ), base swollen, insertion marginal (Figure 33A). Shape elliptic. Apex mucronate (Figures $32 \mathrm{~K}, 33 \mathrm{~A}$ ), shape convex, angle acute. Base shape narrow-decurrent (Figure 33A), angle acute. Primary venation pinnate; primary thicker at the base, course slightly irregular. Basal veins one. Secondary venation mixed craspedodromous and cladodromous; secondaries thin, alternate, arising decurrently at acute angles (Figure $32 \mathrm{M}$ ), angle decreasing toward base (Figure 33A), spacing uniform, course irregular, forking up to two times with irregular anastomoses and loops (Figure 33A-B). Perimarginal venation an irregular intramarginal vein and a fimbrial vein (Figure 33B). Agrophic and intersecondary veins absent. Intercostal tertiary, 

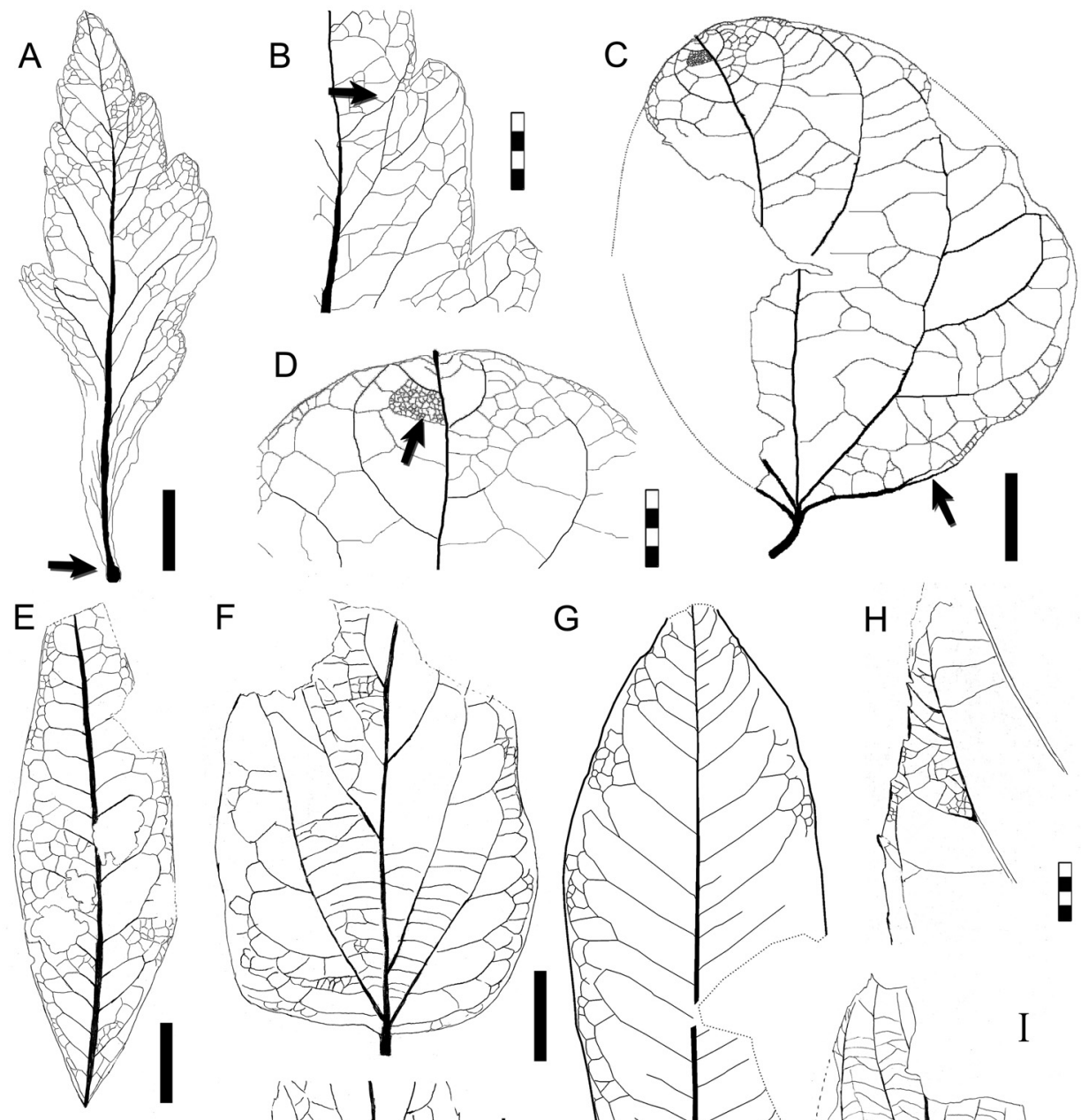

F $\quad G$
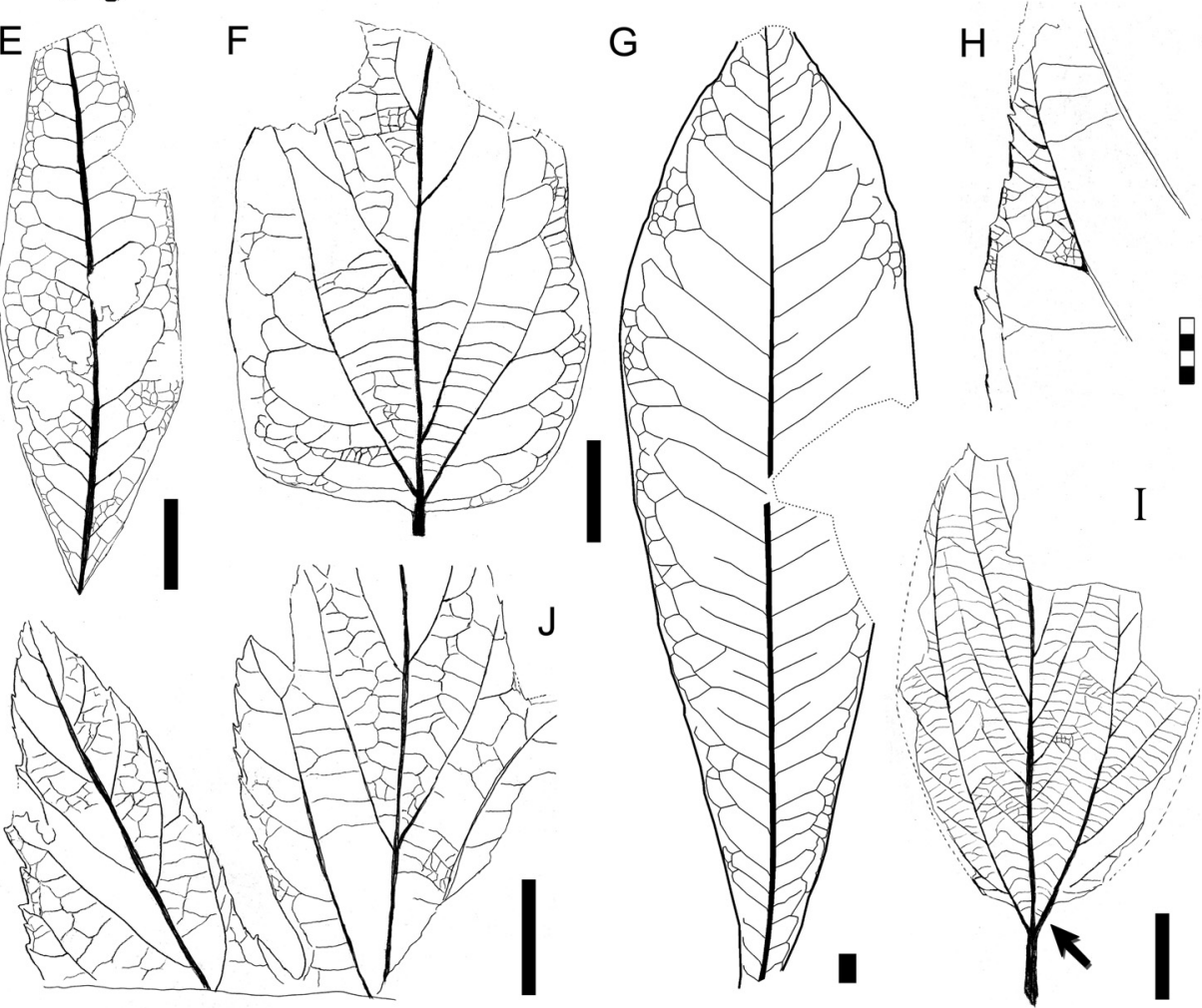

FIGURE 33. Morphotypes SA059 (continued, see also Figure 32K-M), SA060 Wilkinsoniphyllum menispermoides Jud, Gandolfo, Iglesias, and Wilf, 2018 (Menispermaceae, see also Jud et al., 2018 figure 4; and Figure 34A-C), SA063 (see also Figure 34G-I), SA066 (see also Figure 34L-M), SA067 (see also Figure 34D-F), SA068 (see also Figure 35G-K), and SA069 (Rhamnaceae; see also Figure 34J-K). A-B, morphotype SA059, MPEF-Pb-2051 (exemplar, see also Figure 32K-M). A, digital overlay drawing (DOD), note decurrent base shape and swollen petiole base (arrow); B, DOD of tooth venation detail (arrow, anastomosis in venation). C-D, morphotype SA060 W. menispermoides (Menispermaceae), MPEF-Pb-2052 (holotype, see also Figure 34A-C). C, DOD of the entire leaf (arrow, tapering fimbrial vein); D, DOD of mucronate apex and detail of regular, orthogonal higher order venation (arrow). E, morphotype SA063, camera lucida drawing (CLD) of MPEF-Pb-3002 (exemplar, see also Figure 34G, 34I). F, morphotype SA066, CLD of MPEF-Pb-3004 (exemplar, see also Figure 34L-M). G, morphotype SA067, CLD of MPEF-Pb-3006 (exemplar, see also Figure 34D-F). H-I, morphotype SA069, MPEF-Pb-3010 (exemplar, see also Figure 34J-K). H, CLD of tooth venation; I, CLD of the complete leaf (arrow, naked basal veins). J, morphotype SA068, CLD of MPEF-PB-3008 (accessory exemplar, see also Figure $35 \mathrm{G}-\mathrm{H}$ ). Single-color scale bars equal $10 \mathrm{~mm}$; grid scales equal one millimeter (per rectangle). 
epimedial tertiary, fourth, and fourth and fifth order venation random polygonal reticulate (Figure 33A). Areolation well developed. Freely ending ultimate veins one-branched. Vein density $6.98 \mathrm{~mm} / \mathrm{mm}^{2}$ (one leaf measured). Marginal ultimate venation joining the fimbrial vein. Foliar rank $2 r$. Teeth compound, large, with one to three orders, very small at leaf base (Figure 32L); tooth apex with a small opaque spherule (Figure 32M). Tooth shape convex/convex, convex/flexuous or straight/straight, with angular sinuses (Figures 32L-M, 33B). Tooth spacing irregular. Tooth venation variable, comprised of a principal vein derived from a secondary vein that reaches the tooth apex, two accessory veins running near both tooth flanks (Figures $32 \mathrm{M}$, 33B), and a well-defined fimbrial vein.

Observations. This morphotype is based on a single specimen from quarry PL2 (Table 1), but it has several distinctive characters such as its narrowdecurrent base, basally swollen petiole, low foliar rank, and large, compound teeth.

\section{Morphotype SA060}

Menispermaceae Jussieu, 1789 nom. cons. Wilkinsoniphyllum menispermoides Jud, Gandolfo, Iglesias, and Wilf, 2018.

Exemplar. MPEF-Pb-2052 from quarry PL1 (holotype of Wilkinsoniphyllum menispermoides; Jud et al., 2018b, figure 4).

\section{Figures 33C-D, 34A-C}

Description (after Jud et al., 2018b). Blade medially asymmetrical, size notophyll, length $6.0 \mathrm{~cm}$, width $\sim 6.0 \mathrm{~cm}$. Length:width ratio $1: 1$ (one leaf measured). Margin entire. Petiole thin, simple, apparently short (4.3 mm long, 1.7-0.7 mm wide); insertion marginal, pulvinate (wider at the insertion point). Shape wide ovate. Apex shape rounded, characterized by a short mucro $(0.5 \mathrm{~mm}$ long, Figures 33D, 34B) formed by the projecting midvein, angle obtuse. Base shape concavo-convex, angle obtuse, symmetrical. Primary venation suprabasal actinodromous; central primary slightly wider the than two lateral primaries, reaching the apex with strength and projecting as the short mucro; lateral primaries ascending about five-sixth the distance to apex. Basal veins five, three primaries and two secondary marginal veins that join agrophic loops. The two lateral primaries curved toward apex, reaching $2 / 3$ of blade length (Figure 33C). Agrophic veins simple, forming brochidodromous loops. Secondary venation weak brochidodromous; secondary veins excurrent, ascending from the central primary with the lowermost pair diverg- ing near the middle of the blade, central secondary veins alternate, course curved, becoming parallel to margin, joining supradjacent vein by serial loops. Secondary vein angle uniform, spacing increasing toward base. Perimarginal venation a tapering fimbrial vein (Figure 33C). Intersecondary veins absent. Intercostal tertiary venation mixed opposite-alternate percurrent, joining to secondaries at obtuse angles (Figures 33C, 34A). Epimedial tertiary venation alternate percurrent; tertiary vein course generally straight, perpendicular to midvein, angles increasing exmedially. Fourth and fifth order venation regular polygonal reticulate (Figure 33D). Sixth order venation thick, regular polygonal reticulate (Figure 33D). Freely ending veinlets not observed. Vein density $12.02 \mathrm{~mm} / \mathrm{mm}^{2}$ (one leaf measured). Areolation well developed, areoles four-five sided, size uniform. Marginal ultimate venation a thin fimbrial vein.

Observations. As discussed by Jud et al. (2018b; see also Iglesias et al., 2007), distinctive features of Wilkinsoniphyllum menispermoides include its ovate asymmetrical blade shape, mucronate apex, pulvinate petiole, actinodromous primary venation, brochidodromous secondary venation, deeply curved secondary veins, fimbrial vein, and uniform high order venation lacking freely ending veinlets. So far, the leaf species is known only from a single specimen at the PL1 quarry (Table 1). The presence of Menispermaceae in the Salamanca Formation was further confirmed by an endocarp fossil from the PL2 quarry assigned to Stephania psittaca Jud and Gandolfo, 2018 (Jud et al., 2018b). These fossils currently represent the oldest record of Menispermaceae globally (Jud et al., 2018b).

\section{Morphotype SA063}

Affinity. Unknown.

Exemplar. MPEF-Pb-3002 from quarry OR2 (Figures 33E, 34G, 34I).

Figures 33E, 34G-I

Description. Blade symmetrical, size microphyll to notophyll, generally microphyll, mean length 5.0 $(2.4-7.6) \mathrm{cm}$, mean width $2.1(1.2-4.3) \mathrm{cm}$. Mean length:width ratio 2.5:1 (1.9:1-3.8:1). Margin entire. Petiole robust (Figure $34 \mathrm{H}$ ), curved, $0.6 \mathrm{~cm}$ long and $1 \mathrm{~mm}$ wide, insertion marginal. Shape elliptic. Apex shape convex, angle acute (Figure $34 \mathrm{H}$ ). Base shape slightly convex or decurrent, angle acute (Figure $34 \mathrm{H}-\mathrm{I}$ ). Primary venation pinnate; midvein very thick, course straight with relief preserved. Basal veins one (Figure 33E). Secondary venation festooned brochidodromous with weak loops; secondary veins opposite, thickness moder- 


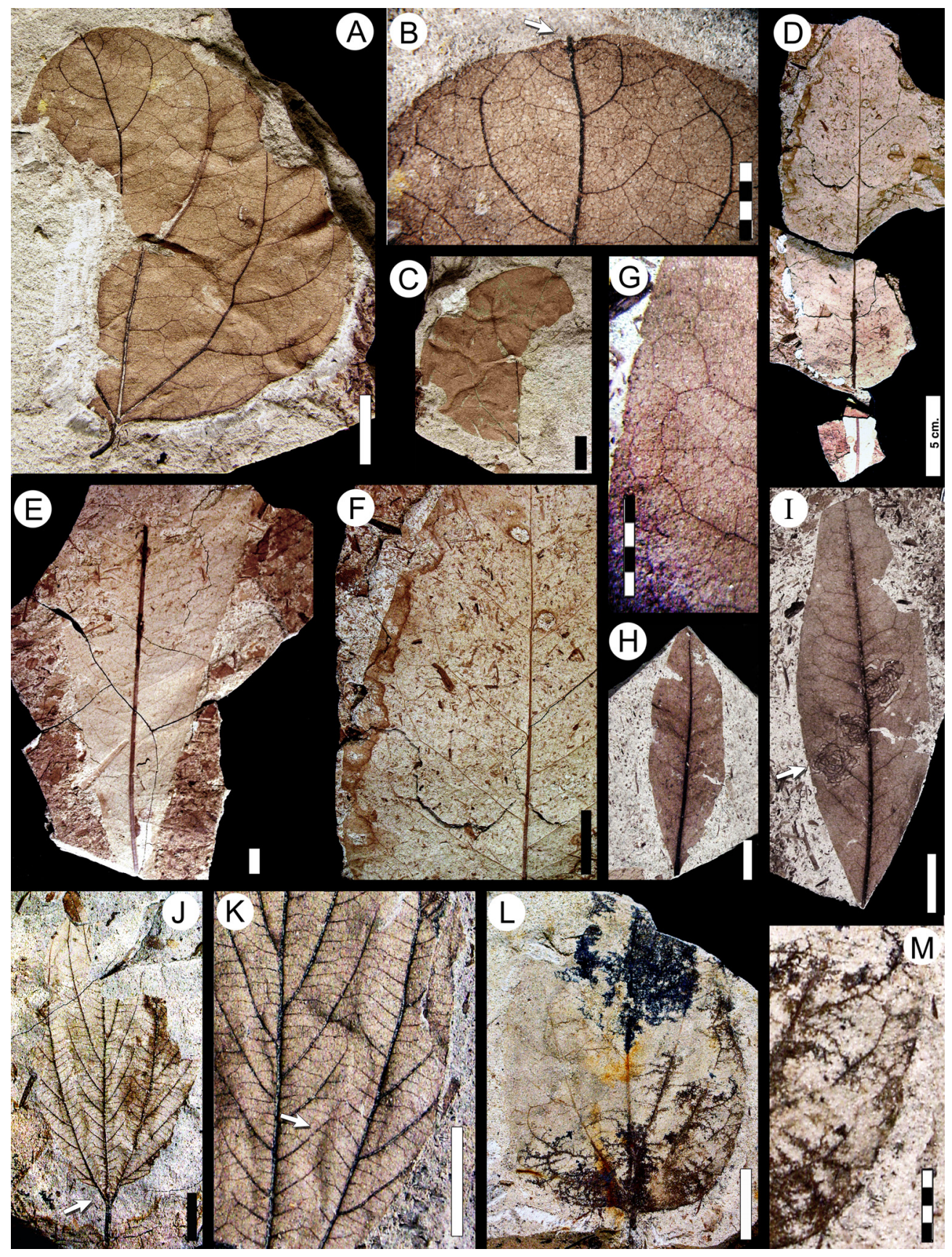

FIGURE 34. Morphotypes SA060 Wilkinsoniphyllum menispermoides Jud Gandolfo, Iglesias, and Wilf, 2018 (Menispermaceae; continued, see also Jud et al., 2018 figure 4, and Figure 33C-D), SA063 (continued, see also Figure 33E), SA066 (continued, see also Figure 33F), SA067 (continued, see also Figure 33G), and SA069 (Rhamnaceae; continued, see also Figure 33H-I). A-C, morphotype SA060 W. menispermoides (Menispermaceae), MPEF-Pb-2052 (holotype, see also Figure 33C-D). A, complete leaf; B, detail of mucronate apex (arrow) and higher order venation; C, counterpart. D-F, morphotype SA067, MPEF-Pb-3006 (exemplar, see also Figure 33G). D, complete leaf; E, leaf base detail; F, apex venation detail. G-I, morphotype SA063. G, venation detail from specimen in Figure 34I; H, MPEF-Pb9124; I, MPEF-Pb-3002 (exemplar, see also Figure 33E), with galling leaf damage (arrow, DT112, also Labandeira et al., 2007 p.14). J-K, morphotype SA069 (Rhamnacaeae), MPEF-Pb-3010 (exemplar, see also Figure 33H-I). J, complete leaf preserving petiole and naked basal veins (arrow); K, counterpart (arrow, chevroned interior veins). L-M, morphotype SA066, MPEF-Pb-3004 (exemplar, see also Figure 33F). L, complete leaf; M, venation detail along part of the basal margin. Single-color scale bars equal $10 \mathrm{~mm}$; grid scales equal one millimeter (per rectangle). 
ate to thin, forking near the margin, joining supradjacent vein with successive loops at near-right angles (Figures 33E, 34G), spacing uniform. Secondary angle to midvein wide-acute (ca. $80^{\circ}$ ), decreasing toward base. Perimarginal and agrophic veins absent. Intersecondary veins pronounced, proximally and distally parallel to subjacent secondary, longer than half the length of subjacent secondary, frequency one or more per secondary (Figure 33E). Intercostal tertiary, epimedial tertiary, fourth order, and fifth order venation random reticulate (Figure 34G). Freely ending veinlets not observed. Areolation well developed. Marginal ultimate venation looped (Figure 34G).

Observations. Morphotype SA063 differs from other entire-margined, pinnate-veined leaves (morphotypes SA010, SA046, SA056, SA073, SA077, and SA078; see key in supplementary Appendix 2) in having a robust midvein with a straight course, festooned brochidodromous secondary veins with angles that decrease toward the base, and prominent interesecondary veins. This morphotype was found only at quarry OR2 (Table 1), from the Salamanca Formation.

\section{Morphotype SA066}

Affinity. Unknown.

Exemplar. MPEF-Pb-3004 from quarry LF (Figures 33F, 34L-M).

Accessory exemplar. MPEF-Pb-3005 from quarry LF.

Figures 33F, 34L-M

Description. Blade symmetrical; size notophyll, length $5.5 \mathrm{~cm}$, mean width $3.4(2.4-4.4) \mathrm{cm}$. Length:width ratio $\sim 2: 1$. Margin entire. Petiole robust, width $1.0 \mathrm{~mm}$, insertion marginal. Shape ovate. Apex not preserved. Base shape rounded, angle obtuse (Figure 34L). Primary venation pinnate; midvein course straight; two thicker secondaries arise ca. $1 \mathrm{~cm}$ above the base, course curved, reaching $2 / 3$ of blade length. Basal veins three. Agrophic veins simple, looped (Figures 33F, $34 \mathrm{M})$. Secondary venation festooned brochidodromous; secondary veins decurrent, alternate, angle acute and decreasing toward base, spacing irregular (Figure 33F). Perimarginal and intersecondary veins absent. Intercostal tertiary venation opposite percurrent, joining to secondaries perpendicularly (Figure 33F). Epimedial tertiary venation opposite percurrent, course straight, perpendicular to midvein at departure. Fourth and fifth order veins opposite percurrent. Areolation well developed with rectangular areoles $1 \mathrm{~mm}$ in diameter. Marginal ultimate venation looped.
Observations. Morphotype SA066 differs from similar morphotypes (e.g., SA010, see key in Supplementary Appendix 2) in its rounded base shape with obtuse base angle, looped agrophic veins, and festooned brochidodromous secondary venation. This morphotype was found at quarries OR2 (Salamanca Formation) and LF (Peñas Coloradas Formation), at low abundance (Table 1).

\section{Morphotype SA067}

Affinity. Unknown.

Exemplar. MPEF-Pb-3006 from quarry OR2.

Figures 33G, 34D-F

Description. Blade symmetrical, size macrophyll, length $34.1 \mathrm{~cm}$, width $9.8 \mathrm{~cm}$. Length:width ratio 3.6:1 (one leaf measured). Margin entire. Petiole insertion marginal. Texture membranaceous. Shape obovate. Apex shape straight, angle acute (Figure 34D). Base shape straight, narrow-elongate; angle acute (Figures 33G, 34E). Primary venation pinnate; midvein prominent, course straight, losing strength distally (Figure 33G). Basal veins one. Secondary venation weakly brochidodromous to reticulodromous; secondary veins thin, course straight, abruptly curved or forked, alternate to subopposite, spacing decreases toward base, angle increasing towards the base (Figure 33G). Intersecondary veins proximally parallel to subjacent secondary, longer than half the length of the subjacent secondary, distally reticulating, one or fewer per secondary (Figures 33G, $34 \mathrm{~F}$ ). Perimarginal, agrophic, and fimbrial veins absent. Intercostal tertiary, epimedial tertiary, fourth, and fifth order venation random polygonal reticulate. Freely ending veinlets not evident due to preservation. Marginal ultimate venation looped (Figure 33G).

Observations. Morphotype SA067 is known from a single specimen from quarry OR2 (Table 1). Despite the fossil's condition, it shows several characters that easily differentiate this morphotype (see key in supplementary Appendix 2) including: large size, narrow-elongate base, obovate shape, and a primary vein that is substantially thicker than other veins.

\section{Morphotype SA068}

Affinity. Unknown.

Exemplar. MPEF-Pb-3007 from quarry LF (Figure 35J-K).

Accessory exemplars. MPEF-Pb-3008 from quarry LF (Figures 33J, 35G-H), and MPEF-Pb3009 from quarry LF. 
Figures 33J, 35G-K

Description. Blade symmetrical (Figure 35I), size mesophyll to megaphyll, mean length 9.1 (5.0$11.5) \mathrm{cm}$, mean width $8.6(5.4-12) \mathrm{cm}$. Length:width ratio $\sim 1: 1$. Margin serrate. Petiole insertion marginal. Shape wide elliptic in outline (Figure 35I-J), palmately 3-lobed, the lobes compound, similar in development. Lobe shape elliptic to ovate-triangular, apex convex; lobules developed along the lobe base, lobule apex angle acute, shape triangular (Figure 35G). Lobe sinuses rounded, untoothed. Blade apex angle obtuse. Base shape rounded, angle obtuse (Figure 35I). Primary venation basal actinodromous, course straight. Lateral primaries with first pair thin, second pair robust, supporting the two lateral lobes, branching basally to form agrophic veins that supply the basal teeth. Basal veins seven: five primary veins, and two secondaries (Figure 35J). Agrophic veins simple or compound (Figure 35J). Secondary venation craspedodromous, arrangement subopposite. Secondary veins curved, unbranched, angle decreasing toward base, spacing increasing toward base. Sinus-bracing veins well developed, arise from the midvein, forming intramarginal veins on both sides of the sinus (Figure $35 \mathrm{H}$ ). Perimarginal venation a fimbrial vein. Intersecondary and interior secondary veins absent. Intercostal tertiary venation mixed opposite-alternate percurrent, joining secondaries at nearly perpendicular angles (Figure 33J). Epimedial tertiary venation opposite percurrent, perpendicular to primaries at departure, course convex. Fourth and fifth order venation random reticulate. Freely ending veinlets not observed. Areolation well developed. Marginal ultimate venation a fimbrial vein. Teeth simple with narrow apex, simple or with a small opaque apical callosity. Tooth shape concave/flexuous, convex/ convex or flexuous/convex; sinuses rounded. Tooth spacing irregular, teeth more developed toward blade base (Figure 35I). Tooth venation supplied by a principal vein from a secondary or its branches, running medially up to the apical gland; accessory venation looped (Figures 33J, 35K).

Observations. Morphotype SA068 was found only at quarry LF (Peñas Coloradas Formation, Table 1). Its palmate, compound, and toothed lobes distinguish SA068 from similar morphotypes (e.g., morphotypes SA009 and SA075; see key in supplementary Appendix 2).

\section{Morphotype SA069}

Affinity. Rhamnaceae? Jussieu, 1789.

Exemplar. MPEF-Pb-3010 from quarry LF.
Figures $33 \mathrm{H}-\mathrm{I}, 34 \mathrm{~J}-\mathrm{K}$

Description. Blade symmetrical, size notophyll, length $6.8 \mathrm{~cm}$, width $3.5 \mathrm{~cm}$. Length:width ratio $\sim 2: 1$ (one leaf measured). Shape elliptic. Margin crenulate except at base. Petiole robust, length 1.0 $\mathrm{cm}$, width $1.3 \mathrm{~mm}$, insertion marginal. Apex shape not preserved, angle acute. Base shape convex, angle acute. Primary venation basal acrodromous. Basal veins three, all primary veins (Figure 34J). Two lateral primaries are naked at the base (Figures $33 \mathrm{I}, 34 \mathrm{~J}$ ) and run more than $2 / 3$ of the blade length, course smoothly curved. Agrophic veins simple (Figures 33I, 34K). Secondary venation semicraspedodromous, interior basally; secondary veins subopposite to alternate, departure excurrent at acute angles, course smoothly curved, angle increasing toward base, spacing decreasing towards the base, branching exmedially, joining to supradjacent vein by one or a series of loops (Figure $34 \mathrm{~J}$ ). Interior secondary veins at leaf base, convex to chevroned (Figures 33I, 34K). Perimarginal venation a fimbrial vein. Intersecondary veins absent. Intercostal tertiary venation opposite percurrent, prominent, convex, perpendicular to secondaries. Epimedial tertiary venation opposite percurrent, convex, perpendicular to primaries, angle increasing exmedially, spacing uniform, sometimes branching. Fourth and fifth order veins thin, regular polygonal reticulate. Areoles well developed, four-five sided. Freely ending veinlets not observed. Marginal ultimate venation a fimbrial vein. Teeth (crenulations) supplied by tertiary veins or the fimbrial vein (Figures 33H, 34K); tooth flanks straight; sinuses small and rounded.

Observations. Morphotype SA069 is known from a single specimen from quarry LF (Peñas Coloradas Formation, Table 1). The acrodromous primary venation with naked basal veins (Figures 33I, 34J); distinctive chevroned interior secondaries; perpendicular, prominent, opposite percurrent tertiaries; and tooth shape and venation distinguish the morphotype and are features that resemble some Rhamnaceae (e.g., Ziziphus mauritiana Lamarck, 1789). As mentioned earlier, Jud et al. (2017) recently described other ziziphoid-ampelozizyphoid clade (Rhamnaceae) leaves and flowers from the Salamanca Formation's Rancho Grande quarry (Figure 1); the Suessenia grandensis Jud, Gandolfo, Iglesias and Wilf, 2017 leaves have almost no secondary veins and have much thinner tertiary veins than morphotype SA069. The most similar rhamnaceous morphotype in this study, morphotype SA048, is basally asymmetrical and actinodromous with much larger teeth and lacks 

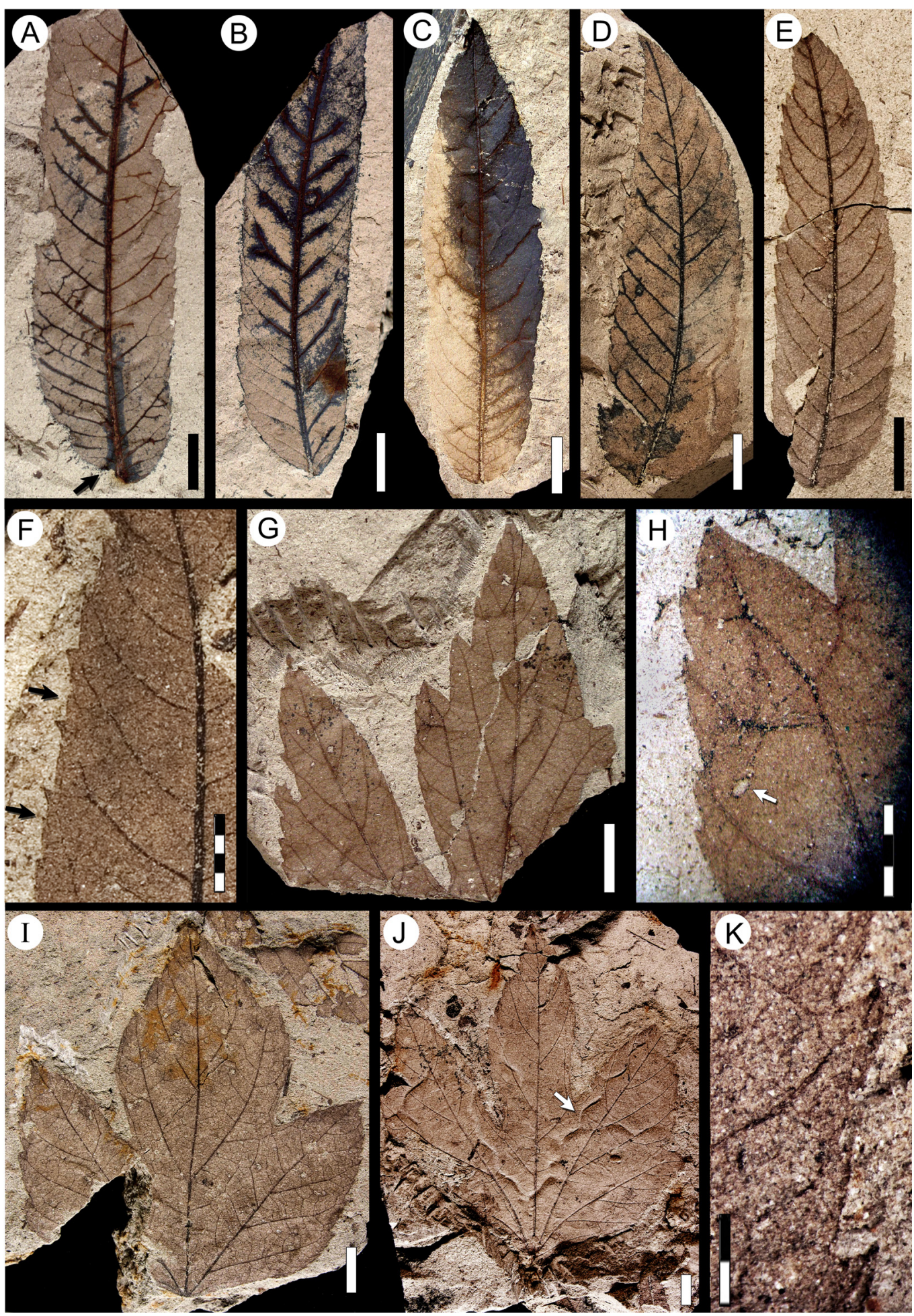

FIGURE 35. Morphotypes SA070 (Juglandaceae? see also Figure 39A-B), SA068 (continued, see also Figure 33J). A-F, morphotype SA070 (Juglandaceae?). A, MPEF-Pb-3012 (accessory exemplar, see also Figure 39A-B), note asymmetric base and basally crowded secondary veins (arrow); B, MPEF-Pb-9125; C, MPEF-Pb-3011 (exemplar); D, MPEF-Pb-3071; E, MPEF-Pb-9126; F, tooth detail of MPEF-Pb-9127 (arrows, second order teeth). G-K, morphotype SA068. G, MPEF-Pb-3008 (accessory exemplar, see also Figure 33J); $\mathrm{H}$, tooth detail around a second-order lobe sinus from specimen in Figure 35G (accessory exemplar), with hole-feeding leaf damage (DT01, arrow); I, MPEF-Pb9128; J, MPEF-Pb-3007 (exemplar), with Cochlichnus trace fossils (arrow); K, tooth detail from specimen in Figure 35J (exemplar). Single-color scale bars equal $10 \mathrm{~mm}$; grid scales equal one millimeter (per rectangle). 
the prominent chevroned veins of morphotype SA069.

\section{Morphotype SA070}

Affinity. Juglandaceae? de Candolle ex Perleb, 1818 nom. cons.

Exemplar. MPEF-Pb-3011 from quarry LF (Figure 35C)

Accessory exemplar. MPEF-PB-3012 from quarry LF (Figures 35A, 39A-B).

Figures 35A-F, 39A-B

Description. Blade asymmetrical, base asymmetrical (Figures 35A-E, 39A). Size notophyll to mesophyll, generally notophyll. Mean length 8.4 (5.710.5) $\mathrm{cm}$, mean width $2.4(1.3-3.4) \mathrm{cm}$. Mean length:width ratio $3.4: 1$ (2.1:1-4.7). Margin serrate. Texture chartaceous to coriaceous. Petiolule (inferred) insertion marginal, disposed at an angle to the midvein. Shape oblong. Apex shape straight, angle acute. Base shape rounded, angle acute. Primary venation pinnate; midvein thick, course slightly curved (Figure 35A-B, 35D-E), wide (width $1 \mathrm{~mm}$ ), reaching the apex with strength. Basal veins three (Figure 39A). Secondary venation craspedodromous to semicraspedodromous (Figure 39B); secondary veins robust, opposite to subopposite, excurrent on midvein at wide angles $\left(45^{\circ}-80^{\circ}\right)$, angles different on either side of blade, increasing toward base; spacing irregular, decreasing and sometimes crowded toward base (Figures $35 \mathrm{~A}, 35 \mathrm{C}, 39 \mathrm{~A}$ ), course slightly curved, branching near the margin. Perimarginal and agrophic veins absent. Intersecondary veins at variable angles to secondaries, shorter than half the length of the subjacent secondary, distally reticulating, one or fewer per secondary (Figures 35D, 39A). Intercostal tertiary venation thin, mixed opposite-alternate percurrent, perpendicular to secondaries. Epimedial tertiary venation opposite percurrent, perpendicular to primaries at departure, course convex to subjacent secondary. Fourth and fifth order venation regular polygonal reticulate (Figure 39A-B). Freely ending veinlets not observed. Areolation well developed, pentagonal in shape, areoles small ( $<0.3 \mathrm{~mm}$ width). Marginal ultimate venation looped. Teeth simple, triangular, in two orders on larger specimens (Figure 35E-F), asymmetrical. Tooth shape straight/convex, straight/straight or flexuous/flexuous; sinuses angular. Tooth spacing irregular, four teeth per centimeter (Figure 39B). Tooth venation supplied by a minor vein branching from a secondary loop, innervating the tooth medially with submarginal termination; accessory venation looped (Figure 39B).
Observations. Morphotype SA070 was found only at quarry LF (Peñas Coloradas Formation, Table 1). Features that resemble Juglandaceae Perleb, 1818 include its oblong and asymmetrical blade, asymmetrical base, serrate margin with simple triangular teeth, pinnate primary venation, thick midvein, craspedodromous and branching secondary venation, secondary vein angles differing on either side of the midvein, thin intersecondaries, and welldeveloped areolation.

\section{Morphotype SA073}

Equivalent to. USNM-201959 syntype of "Cissites patagonica" Berry, 1937 (Berry, 1937a, plate IX, figure 5; here Figure $36 \mathrm{C}$ ) only (Table 2); syntypes USNM-201955, USNM-201956, and USNM201957, excluded (see morphotypes SA009).

Affinity. Unknown.

Exemplar. MPEF-Pb-3031 from quarry LF (Figure 36B).

Accessory exemplar. MPEF-Pb-3032 from quarry LF (Figure 36E).

Figures 36A-E, 39E

Description. Blade symmetrical. Size nanophyll to mesophyll, generally microphyll. Mean length 6.3 $(2.2-10-9) \mathrm{cm}$, mean width $3.1(1.0-8.0) \mathrm{cm}$. Mean Length:width ratio 2.2:1 (1.1:1-3.4:1). Margin entire. Petiole robust, more than $1 \mathrm{~cm}$ in length, insertion marginal (Figure 36A). Shape ovate. Texture chartaceous. Apex shape acuminate, extending into a drip tip, angle acute (Figure 36A-B). Base shape decurrent (Figure 39E), angle acute. Primary venation suprabasal acrodromous; midvein thick, course uniformly curved; two thick lateral primaries arise opposite or subopposite, course slightly curved, running more than $1 / 2$ of the blade parallel to leaf margin tapering apically (Figure 36A-D). Basal veins five; three primary and two secondary veins (Figure 36A-E). Agrophic veins simple, uniformly curved, generating simple loops (Figure 39E). Secondary venation brochidodromous; secondary veins subopposite or subalternate, 4-5 pairs, course uniformly curved, arise in wide and uniform angles, departure from principal veins excurrent, spacing decreasing toward base. The two basal secondaries act as intramarginal veins (Figure 36E) until they joing to agrophic veins in a loop. Perimarginal venation a basal intramarginal vein and a thin fimbrial vein (Figure 36E). Intersecondary veins proximally parallel to the subjacent secondary; shorter than half the length of subjacent secondary, distally reticulating or ramifying, one or fewer per secondary. Intercostal tertiary venation mixed opposite-alternate percurrent, join- 

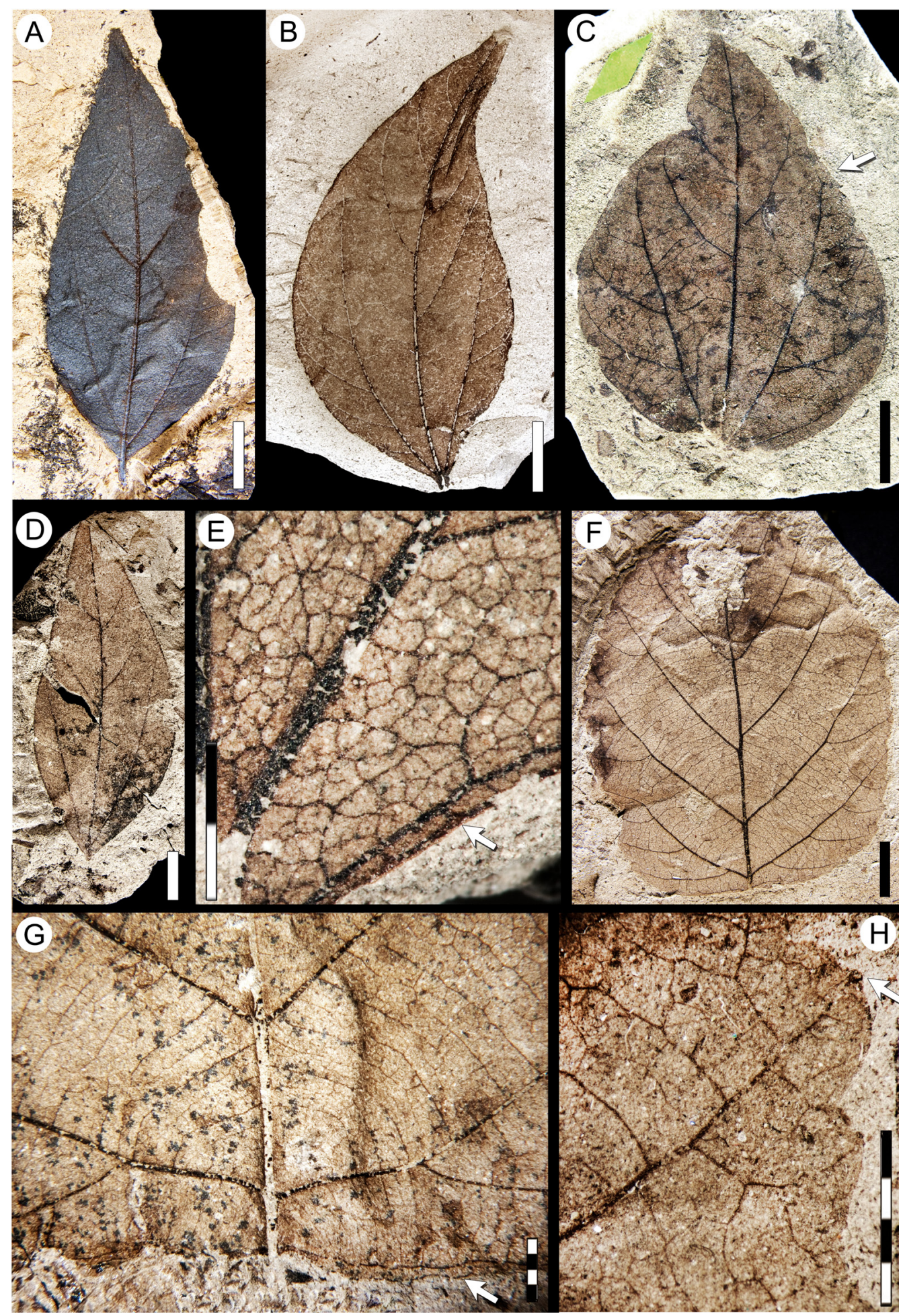

FIGURE 36. Morphotypes SA073 (see also Figure 39E) and SA074 (see also Figure 39F-G). A-E, morphotype SA073. A, MPEF-Pb-9129; B, MPEF-Pb-3031 (exemplar); C, "Cissites patagonica" Berry, 1937a syntype specimen USNM-201959 (illustrated in Berry, 1937a, plate IX, figure 5; see also Table 2), note the extended lateral primary veins (arrow); D, MPEF-Pb-9131; E, higher order venation detail of MPEF-Pb-3032 (accessory exemplar; arrow, fimbrial vein). F-H, morphotype SA074. F, MPEF-Pb-3015 (exemplar, see also Figures 36H, 39F-G); G, venation detail at base of MPEF-Pb-9132, note looped ultimate venation (arrow); $\mathrm{H}$, detail of tooth venation from specimen in Figure $36 \mathrm{~F}$ (exemplar), note glandular tooth apex (arrow). Single-color scale bars equal $10 \mathrm{~mm}$; grid scales equal one millimeter (per rectangle). 
ing to secondaries in acute angles increasing exmedially. Epimedial tertiary venation mixed opposite-alternate percurrent, thin, angle acute to primary increasing exmedially. Fourth and fifth order venation regular polygonal reticulate (Figure $36 \mathrm{E})$. Freely ending veinlets one-branched. Vein density $9.80 \mathrm{~mm} / \mathrm{mm}^{2}$ (one leaf measured). Marginal ultimate venation looped.

Observations. This morphotype is distinguished from other entire-margined, pinnate-veined morphotypes (i.e., SA010, SA046, SA056, SA063, SA077, and SA078) in having well-developed acrodromous secondaries with two simple agrophic veins, a smaller number of secondaries, secondaries with spacing decreasing toward the base, and in the absence of idioblasts (see key in supplementary Appendix 2). Morphotype SA073 has been found only at quarry LF (Peñas Coloradas Formation), where it is abundant ( $18 \%$ of the flora, Table 1).

Morphotype SA073 matches the syntype USNM-201959 for "Cissites patagonica" Berry 1937 (Berry, 1937a, plate IX, figure 5; here Figure $36 \mathrm{C}$ ), although it does not show the same characters listed in Berry's description that are seen in the other three syntypes for that species (see morphotype SA009). USNM-201959 has an untoothed margin, brochidodromous secondary venation, simple agrophic veins, and intramarginal vein that correlate to our morphotype SA073 (Table 2).

\section{Morphotype SA074}

Affinity. Unknown.

Exemplar. MPEF-Pb-3015 from quarry LF (Figures $36 \mathrm{~F}, 36 \mathrm{H}$, 39F-G).

\section{Figures 36F-H, 39F-G}

Description. Blade symmetrical. Size notophyll to mesophyll, generally mesophyll. Mean length 9 $(6.5-12.0) \mathrm{cm}$, mean width $7.2(5.2-9.0) \mathrm{cm}$. Length:width ratio $\sim 1.3: 1$. Margin dentate. Petiole insertion marginal. Shape ovate. Texture membranaceous. Apex shape convex, angle acute. Base shape truncate, angle wide obtuse. Primary venation pinnate, primary vein thickness normal, course straight or deflected, without branching. Basal veins three (Figure 36G). Agrophic veins compound, basal branches curved to reach teeth far from base (Figure 39F). Secondary venation craspedodromous; secondary veins thick, opposite, angle uniform, spacing uniform. Secondaries decurrent on midvein (Figures 36F, 39F). Perimarginal and intersecondary veins absent. Intercostal tertiary venation thin, mixed opposite-alternate percurrent, departing from secondaries at obtuse angles. Epimedial tertiary venation mixed percurrent, course sinuous, obtuse to primary. Tertiary vein form a characteristic concentrical pattern centered around the petiole insertion (Figures 36G, 39F), more distinctive near the leaf base. Fourth order of veins irregular alternate percurrent, course curved (Figure 39F). Fifth order of veins thin, regular polygonal reticulate, course straight. Freely ending veinlets unbranched. Vein density 12.10 $\mathrm{mm} / \mathrm{mm}^{2}$ (one leaf measured). Areolation well developed. Marginal ultimate venation looped. Teeth in two orders, symmetric, well developed along entirety of margin except at base, tooth apex glandular with a callosity fused to the apex (Figures $36 \mathrm{H}, 39 \mathrm{G})$. Tooth shape convex/convex, flexuous/ convex or flexuous/flexuous; sinuses angular. Tooth spacing uniform, two teeth per centimeter. Tooth venation supplied by a midvein from a secondary or its branch, running medially up to the apical gland; accessory venation looped. Sinuses supplied by an independent vein from the secondary or branches (Figures $36 \mathrm{H}, 39 \mathrm{G}$ ).

Observations. Distinguishing characters of this morphotype include: agrophic veins compound; tertiary venation mixed percurrent with a concentrical pattern centered around the petiole insertion; and teeth glandular, uniform in shape and spacing (see key in supplementary Appendix 2). Morphotype SA074 so far is only known from six specimens at quarry LF (Peñas Coloradas Formation, Table 1).

\section{Morphotype SA075}

Affinity. Unknown.

Exemplar. MPEF-Pb-3016 from quarry LF (Figures 37E, 39C).

Figures 37E-H, 39C-D

Description. Blade symmetrical. Size microphyll to mesophyll, generally mesophyll. Mean length 9.5 $(4.5-14.5) \mathrm{cm}$, mean width $10.8(5.0-16.0) \mathrm{cm}$. Length:width ratio $\sim 1: 1$. Margin dentate. Petiole thin, up to $6.2 \mathrm{~cm}$ in length, marginally inserted. Shape elliptic in outline, palmately 3-lobed with several small lobules or teeth. Apex angle obtuse, lobe apex convex. Base shape wide rounded to truncate with smoothly rounded lobes, angle obtuse (Figure 37E, 37G). Primary venation basal actinodromous; primary vein course straight to smoothly curved. Basal veins five, three primaries and two (sometimes three) secondary veins (Figure 39C). Agrophic veins simple, with minor secondaries innervating the basal teeth (Figures 37E, 37G, 39C). Secondary venation craspedodromous; secondary veins thin, subopposite, course curved, 

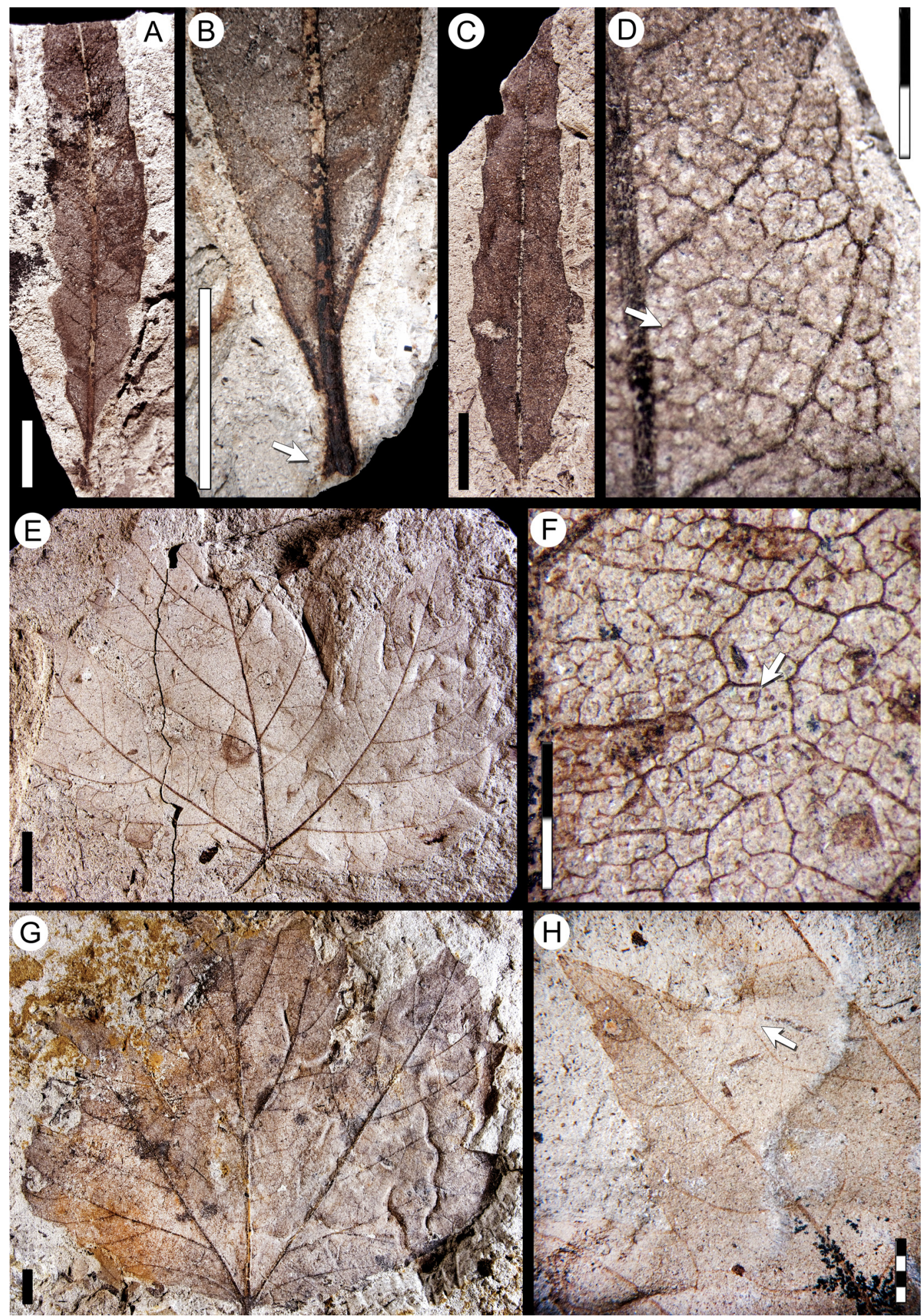

FIGURE 37. Morphotypes SA076 (see also Figure 40A-B) and SA075 (see also Figure 39C-D). A-D, morphotype SA076. A, MPEF-PB-3017 (exemplar, see also Figures 37B, 40A); B, base detail from counterpart of specimen in Figure 37A (arrow, swollen petiole base); C, MPEF-Pb-9135; D, higher order venation detail of MPEF-Pb-9136 (arrow, one-branched freely ending veinlets). E-H, morphotype SA075. E, MPEF-PB-3016 (exemplar, see also Figure 39C); $\mathrm{F}$, higher order venation in MPEF-Pb-9137 (arrow, regular polygonal reticulate higher-order veins); G, MPEF-Pb-9138; $\mathrm{H}$, tooth venation of MPEF-Pb-9139 (arrow, sinus-bracing vein, see also Figure 39D). Single-color scale bars equal 10 $\mathrm{mm}$; grid scales equal one millimeter (per rectangle). 
angle acute uniform, spacing smoothly increasing toward base. Perimarginal venation absent. Intersecondary veins proximally parallel to subjacent secondary, longer than half the length of subjacent secondary; distally reticulating or ramifying; one or fewer per secondary. Sinus-bracing veins moderately developed (Figures $37 \mathrm{H}, 39 \mathrm{C}$ ). Intercostal and epimedial tertiary venation mixed reticulate and weakly percurrent, thin, angle increasing exmedially. Fourth and fifth order venation regular polygonal reticulate. Sixth order of veins thin, regular polygonal reticulate (Figure 37F). Freely ending veinlets not observed. Vein density $10.12 \mathrm{~mm} / \mathrm{mm}^{2}$ (one leaf measured). Areolation well developed. Marginal ultimate venation looped. Teeth compound in two orders, first order teeth large, with several small second order teeth developed on both flanks; small teeth present on the leaf base (Figure 39C). Tooth shape straight/straight or convex/convex (first order teeth) or concave/convex (second order teeth), sinuses rounded. Tooth spacing increasing toward base for both first- and second-order teeth, first-order teeth one per centimeter, second-order teeth six per centimeter (Figure $37 \mathrm{H}$ ). Tooth venation for first-order teeth supplied by a secondary vein reaching the tooth apex medially with straight course, giving off uniformly curved branches to the basal second-order teeth (Figures 37H, 39D).

Observations. Morphotype SA075 was found only at quarry LF (Peñas Coloradas Formation, Table 1). It is easily distinguished by its palmate lobation with several orders of lobes, lobe apex convex, and dentate margin with compound teeth (see key in supplementary Appendix 2).

\section{Morphotype SA076}

Affinity. Unknown.

Exemplar. MPEF-Pb-3017 from quarry LF (Figures 37A-B, 40A).

\section{Figures 37A-D, 40A-B}

Description. Blade symmetrical, base and insertion asymmetrical (Figure 37A-B). Size microphyll, length to $8.0 \mathrm{~cm}$, width to $1.4 \mathrm{~cm}$. Length:width ratio 6:1 (one leaf measured). Margin serrate. Petiole thick and short, base swollen (Figures 37B, $40 \mathrm{~A}$ ); length $6 \mathrm{~mm}$, width $2 \mathrm{~mm}$, marginally inserted. Shape narrow ovate or elliptic. Apex shape convex, angle acute. Base shape straight, angle acute. Primary venation pinnate; midvein thick, course straight, tapering toward apex (Figures $37 \mathrm{C}, 40 \mathrm{~B})$. Basal veins three: one primary vein and two fimbrial veins (Figure 37B). Agrophic veins absent. Secondary venation craspedodro- mous; secondary veins subopposite, angle acute and slightly irregular, smoothly increasing toward base, spacing irregular, may branch near margin, innervating corresponding tooth and supradjacent sinus (Figure 40A-B). Perimarginal venation a fimbrial vein. Intersecondary veins thin, weak, proximally parallel to subjacent secondary; shorter than half the length of subjacent secondary, frequency less than one per secondary vein. Intercostal and epimedial tertiary, fourth, and fifth order venation random reticulate. Freely ending veinlets onebranched (Figure 37D). Areolation well developed. Marginal ultimate venation looped, with fimbrial vein thicker near base. Teeth small, simple, very asymmetrical. Tooth shape concave/flexuous or concave/convex, sinuses long-rounded. Tooth spacing uniform, one per centimeter. Tooth venation supplied by a secondary medial vein reaching the apex; accessory veins looped (Figure 37D).

Observations. Distinguishing characters of this morphotype include: secondary veins irregular and teeth small and widely spaced, with long-rounded sinuses (see key in supplementary Appendix 2). Morphotype SA076 was found only at quarry LF (Peñas Coloradas Formation, Table 1).

\section{Morphotype SA077}

Affinity. Unknown.

Exemplar. MPEF-Pb-3018 from quarry LF (Figures 38A, 38D, 40C).

\section{Figures 38A-D, 40C}

Description. Blade asymmetrical. Size microphyll to notophyll, generally microphyll. Mean length 6.4 $(4.0-8.5) \mathrm{cm}$, mean width $3.9(1.4-5.6) \mathrm{cm}$. Mean length:width ratio 1.7:1 (1.4:1-2.2:1). Margin entire. Petiole insertion marginal. Shape ovate. Apex shape acuminate, angle acute (Figures 38A-C, 40C). Base shape convex, angle obtuse (Figure $38 \mathrm{C}$ ). Primary venation pinnate; primary vein thick, course nearly straight, unbranched. Basal veins one. Agrophic veins absent; basal exterior tertiaries looped (Figures 38A, 40C). Secondary venation festooned weak-brochidodromous, departure from midvein excurrent, angles $\sim 60^{\circ}$, angle smoothly decreasing toward base; spacing irregular, increasing toward base; secondary vein course nearly straight, forking near the margin (Figure 38B), generating loops that join supradjacent vein at perpendicular angles, usually generating second order loops (festoons). Perimarginal and intersecondary veins absent. Intercostal tertiary venation opposite percurrent, weak, joining secondaries at acute angles (Figure 38D). Epimedial tertiary venation opposite percurrent, weak, course straight or 


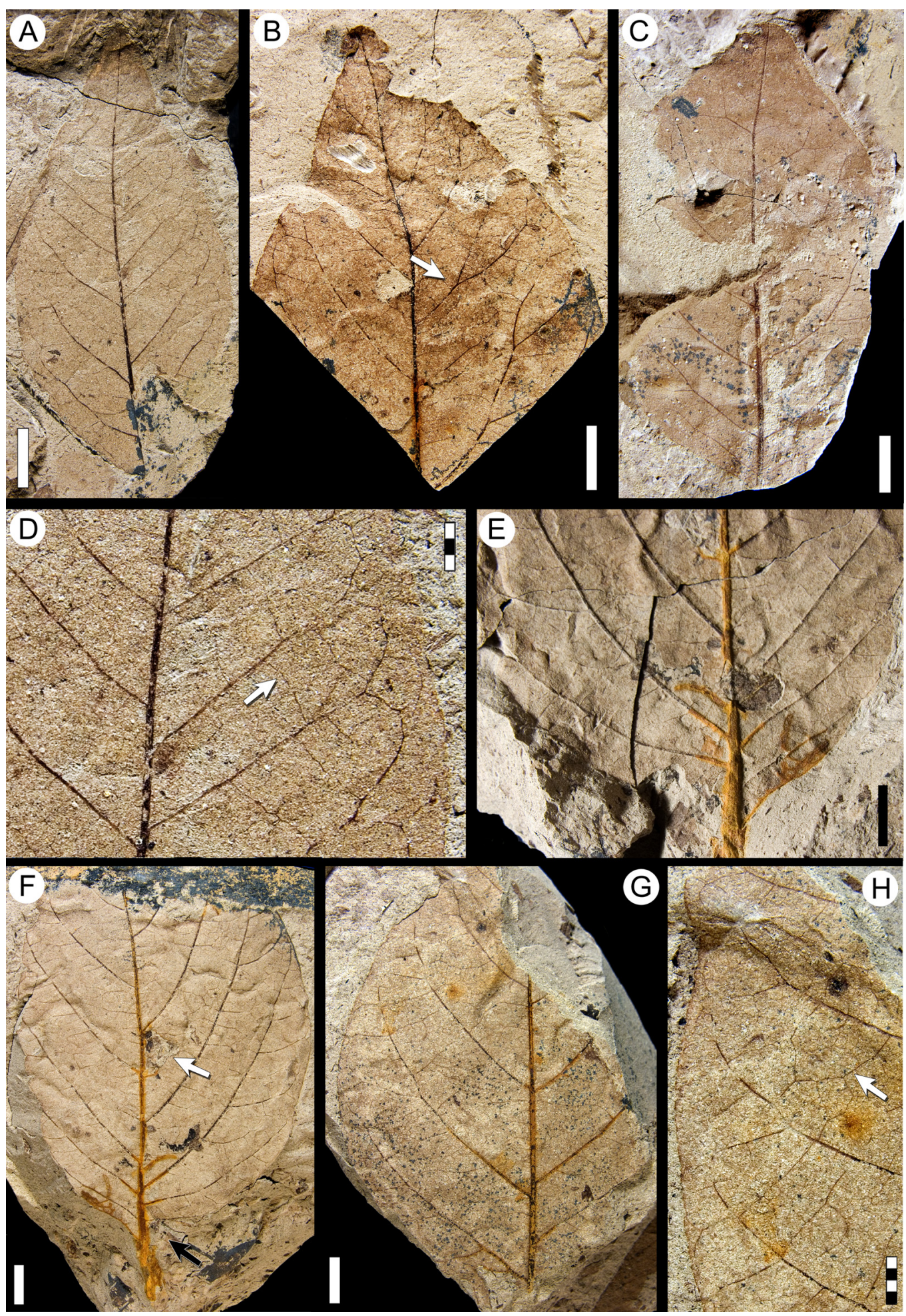

FIGURE 38. Morphotypes SA077 (see also Figure 40C), and SA078 (see also Figure 40E). A-D, morphotype SA077. A, MPEF-Pb-3018 (exemplar, see also Figure 40C); B, MPEF-Pb-9140 (arrow, forked secondary vein); C, MPEF-Pb9141; D, venation detail from Figure 38A (exemplar; arrow, percurrent tertiary vein). E-H, morphotype SA078. E, base venation from counterpart of specimen in Figure 38F (exemplar); F, MPEF-Pb-3019 (exemplar, see also Figures 38E, $40 \mathrm{E}$ ), note asymmetrical blade and base (black arrow) and large polylobate hole damage (white arrow DT5; see also Donovan et al., 2018 figure 13.4); G, MPEF-Pb-9142; H, venation detail from Figure 38G (arrow, alternate percurrent tertiary veins). Single-color scale bars equal $10 \mathrm{~mm}$; grid scales equal one millimeter (per rectangle). 

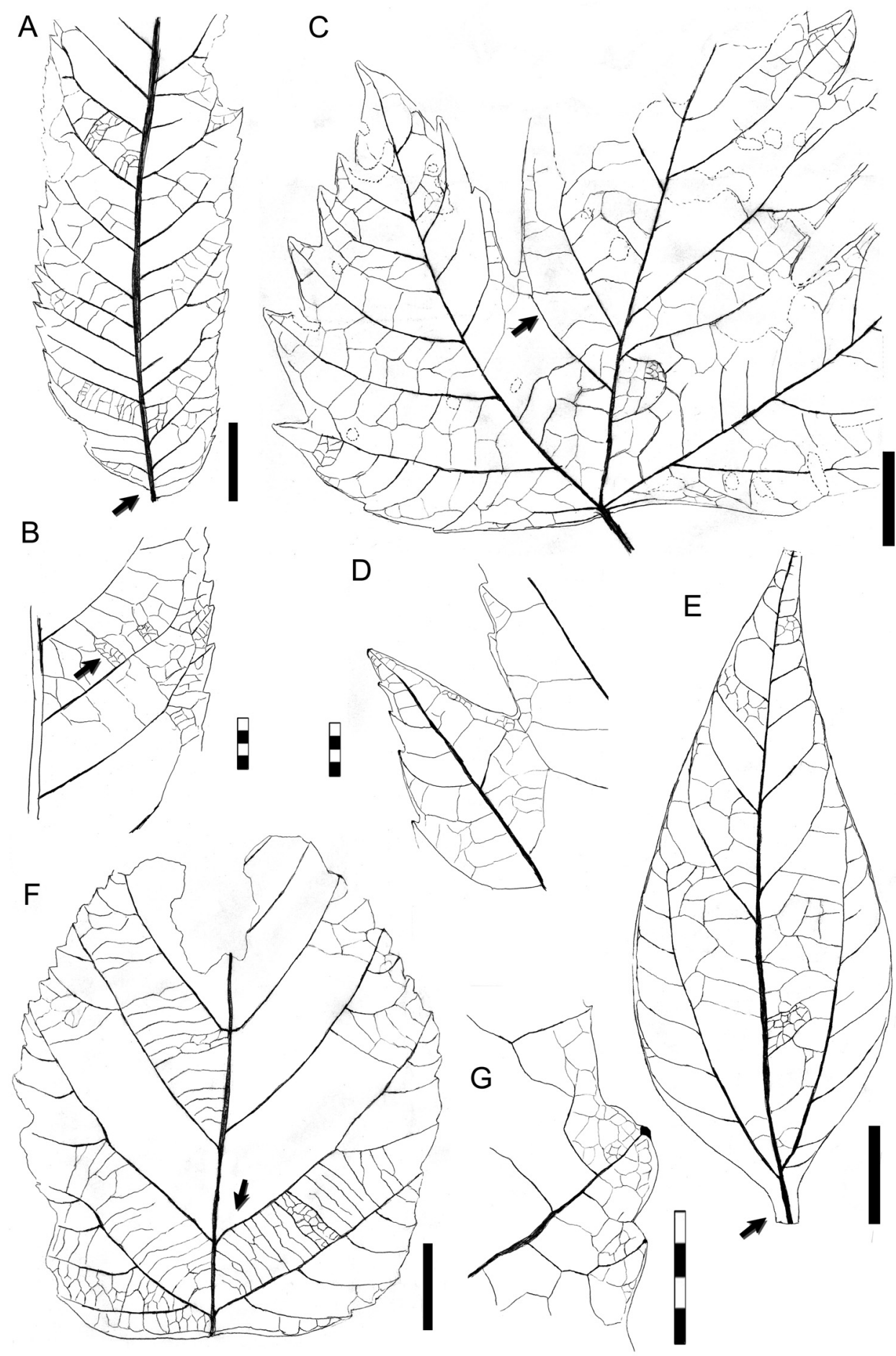
convex, angle variable (Figure 40C). Fourth- and fifth-order veins random reticulate. Areolation well developed. Freely ending veinlets not observed. Marginal ultimate venation looped.

Observations. Morphotype SA077 differs from other entire-margined, pinnate-veined leaves (Morphotypes SA010, SA046, SA056, SA063, SA073, and SA078) in having an asymmetrical blade, festooned, weak brochidodromous secondary venation, and an acuminate apex (see key in supplementary Appendix 2). Morphotype SA077 was found only at quarry LF (Peñas Coloradas Formation, Table 1).

\section{Morphotype SA078}

Affinity. Unknown.

Exemplar. MPEF-Pb-3019 from quarry LF (Figures 38E-F, 40E).

\section{Figures 38E-H, 40E}

Description. Blade and base asymmetrical (Figure 38F). Size mesophyll. Mean length 12.5 (12.013.5) $\mathrm{cm}$, mean width $8.0 \quad(7.8-8.4) \mathrm{cm}$. Length:width ratio $\sim 1.5: 1$. Margin entire. Petiole thick, almost $2.3 \mathrm{~cm}$ in length and $2.7 \mathrm{~mm}$ wide; marginally inserted (Figures 38E-F, 40E). Shape broad elliptic. Apex shape not preserved, angle acute. Base shape convex, angle obtuse. Primary venation pinnate; primary vein thick, course nearly straight. Basal veins three, one medial vein and two secondaries that initiate the fimbrial vein (Figure 40E). Agrophic veins compound, generating brochidodromous basal branches. Secondary venation brochidodromous; secondary veins thick, alternate, course smoothly curved, angle increasing toward base, spacing decreasing toward base, basal secondaries congested and more obtuse (Figures 38F, 40E). Perimarginal venation a fimbrial vein arising from the base (Figure 40E). Intersecondary veins absent. Intercostal tertiary venation mixed opposite-alternate percurrent (Figures $38 \mathrm{H}, 40 \mathrm{E}$ ), approximately perpendicular to secondaries, angle decreasing exmedially. Epimedial tertiary venation alternate percurrent, course straight, perpendicular to primary. Fourth and fifth order veins random reticulate (Figure 40E). Areolation well developed. Marginal ultimate venation looped.

Observations. Morphotype SA078 differs from other entire-margined pinnate-veined morphotypes (i.e., SA010, SA046, SA056, SA063, SA073, and SA077) in having a thick petiole, asymmetric base, basally crowded and irregular secondaries, and compound agrophic veins (see key in supplementary Appendix 2). This morphotype was found only at quarry LF (Peñas Coloradas Formation, Table 1).

\section{MONOCOT LEAF MORPHOTYPES}

\section{Morphotype SA032}

Affinity. Arecaceae Berchtold and Presl, 1820.

Exemplar. MPEF-Pb-3021 from quarry PL1 (Figure 41A).

Accessory exemplar. MPEF-Pb-3022 from quarry PL1 (Figure 41B).

Figure 41A-C

Description. Blade fragments linear, elongate, plicate (Figure 41A), preserved length to $20 \mathrm{~cm}$, width to $5 \mathrm{~cm}$. Preserved margin entire (Figure 41A-B). Texture chartaceous. Base and apex not preserved. Venation parallel in two orders ( $A$ and $B$ ), with one to five $B$ veins per $A$ vein (Figure $41 C$ ). The $A$ veins are spaced approximately $0.7 \mathrm{~mm}$ apart, nine to 11 per segment, with course very straight; the $B$ veins have a straight course with deflections from transverse veins; the transverse veins variably angled with course straight, convex, or sinuous.

Observations. The plicate segments, marked midvein in the basal portion of the leaf, and two orders of lateral veins are characteristic of Arecaceae (Read and Hickey, 1972). The well-marked midvein is present only in one specimen (MPEF-Pb-3021, Figure 41A). Specimens of morphotype SA032 have linear segments with nine and 11 lateral veins, with transverse veins at variable angles that differentiate morphotype SA032 from other palm morphotypes in this work. Although very fragmented, this morphotype was identified through all Danian time intervals considered here (Table 1).

\section{Morphotype SA061}

Affinity. Araceae? Jussieu, 1789.

Exemplar. MPEF-Pb-3000 from quarry PL2 (Figures 40G, 41H-K).

Accessory exemplar. MPEF-Pb-3001 from quarry PL2.

Figures 40G, 41H-K

Description. Blade fragments wide, size probably macrophyll, length at least $20 \mathrm{~cm}$, width almost 6 $\mathrm{cm}$. Margin entire. Venation pinnate (Figure 41I). Primary venation parallel-pinnate. Midrib very thick, $0.6 \mathrm{~cm}$ wide, comprised of multiple bundles ("costa" sensu Hickey and Peterson, 1978; Figure $41 \mathrm{I}, 41 \mathrm{~K})$, with a thicker area of surrounding accompanying tissue (keel?, arrows in Figure $41 \mathrm{~K}$ ). Lateral (secondary) veins eucamptodro- 

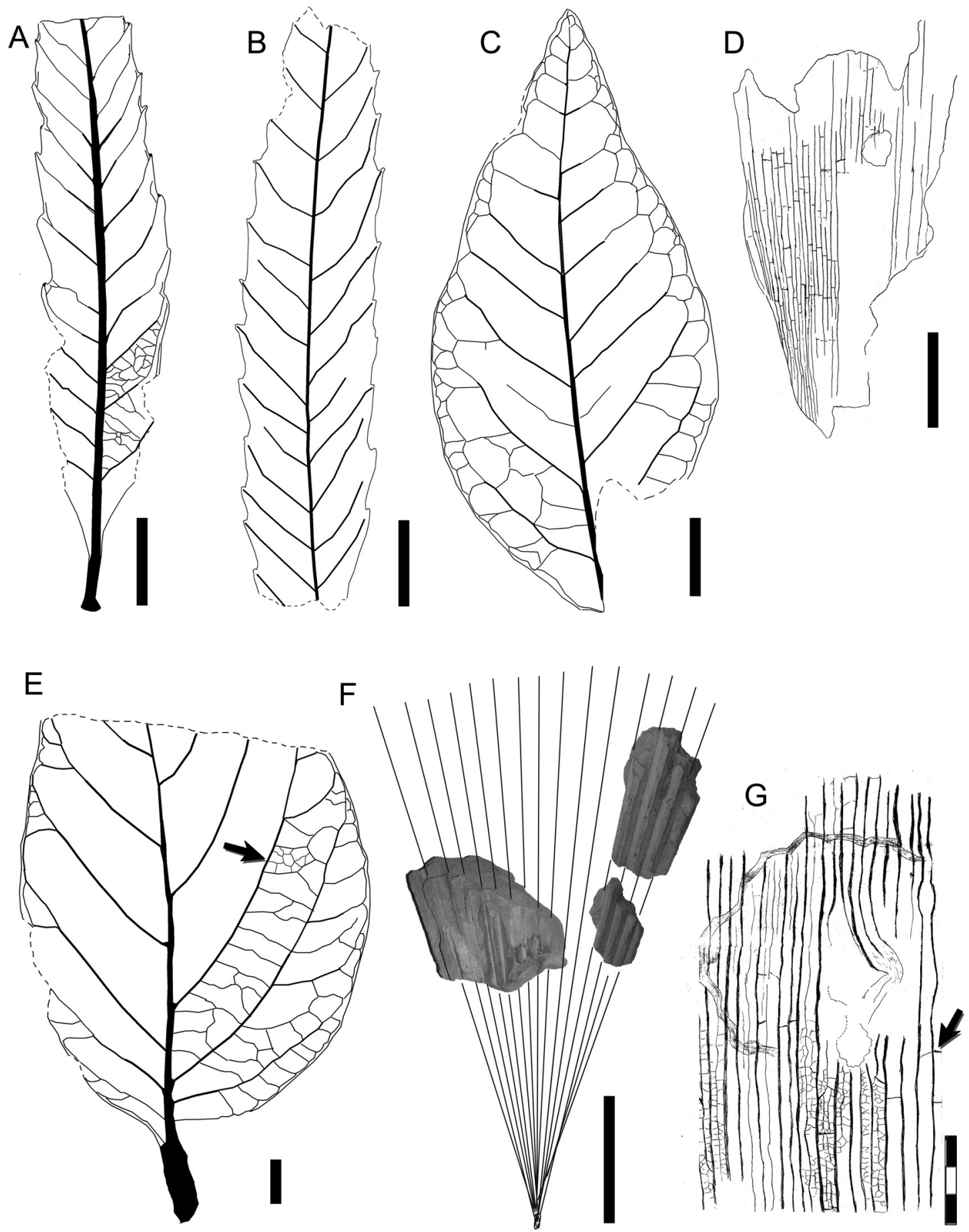

FIGURE 40. Morphotypes SA061 (Araceae? see also Figure 41H-K), SA065 (Arecaceae, see also Figure 41F-G), SA072 (Arecaceae, see also Figure 41D-E), SA076 (continued, see also Figure 37A-D), SA077 (continued, see also Figure 38A-D), SA078 (continued, see also Figure 38E-H). A-B, morphotype SA076. A, digital overlay drawing (DOD) of MPEF-Pb-3017 (exemplar, see also Figure 37A-B); B, DOD of MPEF-Pb-9153. C, morphotype SA077, DOD of MPEF-Pb-3018 (exemplar, see also Figure 38A, 38D). D, morphotype SA065 (Arecaecae), camera lucida drawing (CLD) of MPEF-Pb-3003 (exemplar, see also Figure 41F-G). E, morphotype SA078, DOD of MPEF-Pb-3019 (exemplar; see also Figure 38E-F) (arrow, reticulate higher-order veins). F, reconstruction of morphotype SA072 (Arecaceae) based on three leaf samples (MPEF-Pb-3013, -3014, and -4155). G, morphotype SA061 (Araceae?), CLD of MPEF$\mathrm{Pb}-3000$ (exemplar; see also Figure 41H-K; arrow, transverse veins). Scale bars equal $10 \mathrm{~mm}$, except $3 \mathrm{millimeter}$ scale in Figure 40G. 


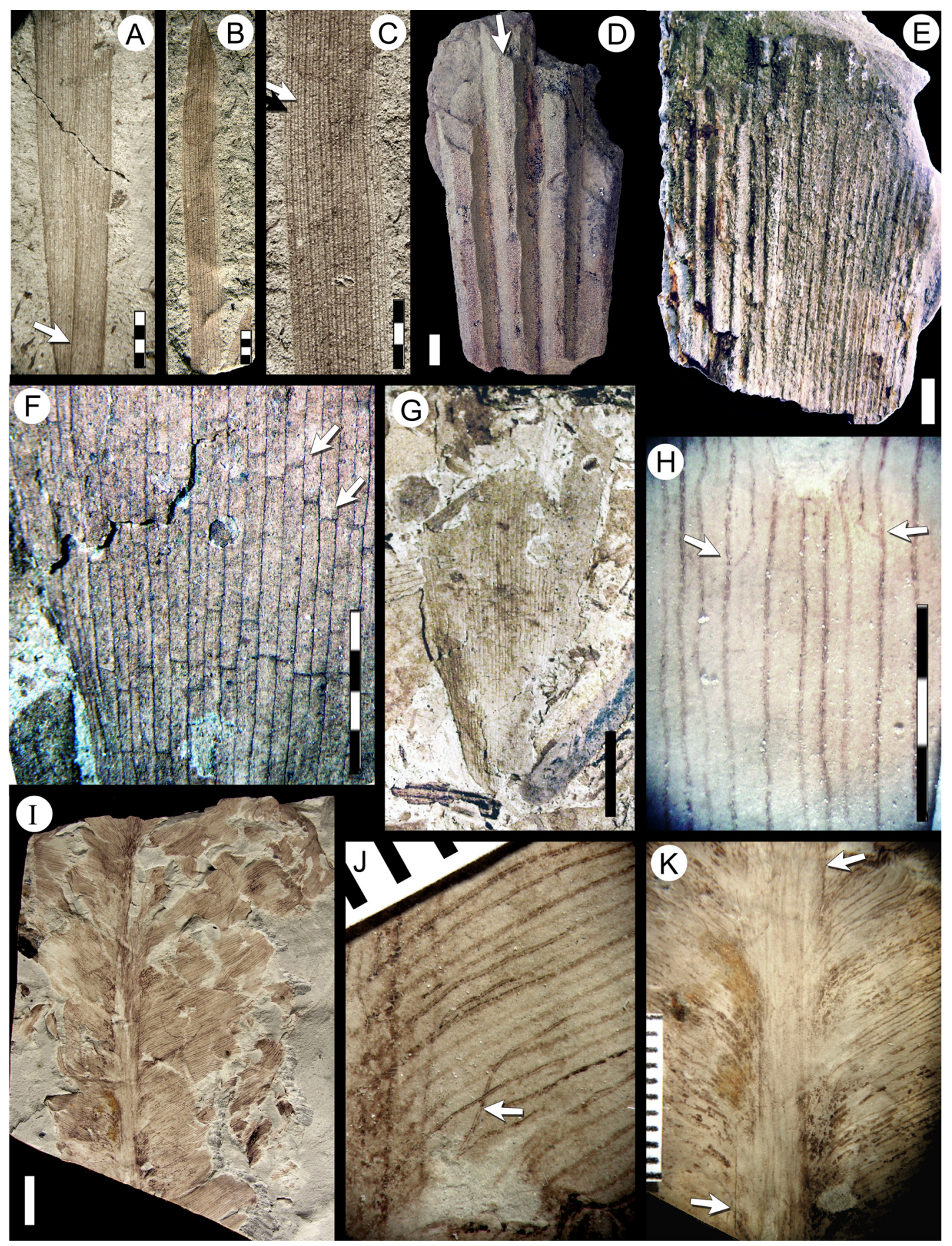

FIGURE 41. Monocot leaf morphotypes of Araceae? (SA061 continued, see also Figure 40G) and Arecaceae (SA032; SA065, continued, see also Figure 40D; SA072, continued, see also Figure 40F). A-C, morphotype SA032 (Arecaceae). A, MPEF-Pb-3021 (exemplar; arrow, segment plication); B, MPEF-Pb-3022 (accessory exemplar); C, MPEF-Pb-9144 (black arrow, A veins; white arrow B veins). D-E, morphotype SA072 (Arecaceae). D, MPEF-Pb-3013 (exemplar), note the flattened carena (arrow); E, MPEF-Pb-3014 (accessory exemplar). F-G, morphotype SA065 (Arecaceae), MPEF-Pb-3003 (exemplar, see also Figure 40D). F, detail of the segment venation (arrows indicating straight, sinuous or oblique transverse veins); G, detail of a segment. H-K, morphotype SA061 (Araceae?), MPEF-Pb3000 (exemplar, see also Figure 40G). H, detail showing vein anastomoses (arrows) and higher order venation; I, complete view of fossil; J, detail of fibers (arrow) accompanying lateral veins; $\mathrm{K}$, detail of massive midvein, with thick surrounding tissue (arrows). Single-color scale bars equal $10 \mathrm{~mm}$; grid scales equal one millimeter (per rectangle). 
mous, in multiple orders (Figures 40G, 41H); densely spaced, about 20 per centimeter; thin, parallel, strongly decurrent on primary, angle $80-90^{\circ}$ to midvein (Figure 41J-K); major lateral veins course straight, abruptly curved near the margin and joining in a perimarginal vein (intramarginal); minor lateral veins parallel to major laterals, sometimes dichotomizing or anastomosed in acute angles (Figures 40G, 41H). Intramarginal vein running parallel to the margin. Strengthening tissue as fibers (probably bundle sheath or caps) accompany major order lateral veins (Figure 41J), with 16 major strengthened lateral veins per centimeter. Transverse veins percurrent at ca. $80^{\circ}$ to lateral veins, in several orders (major veins spacing about $1.7 \mathrm{~mm}$ ), course straight, forming a rectangular reticulate pattern, forming rows of areoles between lateral veins (Figures 40G, 41H). Vein density 4.82 $\mathrm{mm} / \mathrm{mm}^{2}$ (one leaf measured). Texture chartaceous to membranaceous.

Observations. Morphotype SA061 was found only at the OR2 and PL2 quarries, at low abundance (Table 1). The minor-order veins perpendicular to principal veins or at wide angles $\left(\sim 80^{\circ}\right)$ are found in leaves of the order Zingiberales Grisebach, 1854 (Hickey and Peterson, 1978; Fischer et al., 2009); however, the fossil differs in having noticeable fibers accompanying lateral veins. Strengthening tissue (bundle sheath or caps) accompanying major order lateral veins and midribs with surrounding tissue as a keel are common in aroids (e.g., Orontoideae Mayo, Bogner, and Boyce, 1997, Pothoideae Keating, 2002).

\section{Morphotype SA065}

Affinity. Arecaceae Berchtold and Presl, 1820. Exemplar. MPEF-Pb-3003 from quarry OR2.

Figures 40D, 41F-G

Description. Fragment of leaf basal segment, length at least $4 \mathrm{~cm}$, width almost $3 \mathrm{~cm}$ (one leaf measured), shape of fragment triangular (Figure $41 \mathrm{G}$ ), preserved with a slightly curved surface. Margin entire and straight. Venation consists of one order of parallel veins and transverse veins. Midrib indistinguishable. Parallel venation radiating from the segment base, spacing $0.7 \mathrm{~mm}$, course uninterrupted. Transverse veins in two orders of thickness, mostly perpendicular, some oblique (Figures 40D, 41F), mostly straight, some sinuous or convex. Major transverse veins stratified at same blade level spaced $6 \mathrm{~mm}$. apart (Figure 40D).

Observations. Morphotype SA065 is represented by a single fragmentary fossil from the OR2 quarry
(Salamanca Formation). The apparent curvature, base shape, entire margin, and transverse venation suggest that morphotype SA065 is a base segment from a fossil palm. Morphotype SA065 differs from other palm morphotypes in this study based on the triangular segment shape, single order of parallel veins, and major transverse veins. Only few palm genera with pinnate compound leaves (e.g., Iriartea Ruiz and Pavón, 1797; Normanbya Mueller, 1858; Podococcus Mann and Wendland, 1864; and Socratea Karsten, 1856) have midribs that are indistinguishable from other single order lateral veins.

\section{Morphotype SA072}

Affinity. Arecaceae Berchtold and Presl, 1820.

Exemplar. MPEF-Pb-3013 from quarry CP (Figure 41D).

Accessory exemplar. MPEF-Pb-3014 from quarry CP (Figure 41E).

Figures 40F, 41D-E

Description. Blade flabellate (Figure 40F), strongly plicate (Figure 41D-E). Venation parallel. Segments fused, plicate, with a strong carena, rectangular in section (Figure 41D), $2 \mathrm{~mm}$ in width, $0.5 \mathrm{~mm}$ tall, margin straight, and thickened. Segment preserved length at least $45 \mathrm{~cm}$, width variable. Segments radiating at angles of $2.6^{\circ}$, fused, spacing uniform.

Observations. Morphotype SA072, the only strongly plicate and flabellate palm morphotype, is known from four fragmentary fossils from Cerro de las Palmeras (CP) quarry, at the Ormachea Petrified Forest Park locality (correlated to OR2 in Table 1). The fossils are preserved as casts in a coarse sand matrix with iron cement. Three specimens (Figure 41D-E) preserve the segments' strong ridge (rectangular), which indicate the induplicate nature for this leaf and restrict morphotype SA072 to the induplicate palm groups (Moore and Uhl, 1982). Projecting lines between the segment axes and angles from the associated leaf fragments, we have reconstructed a minimum blade length of 45 $\mathrm{cm}$ (Figure 40F) and interpret that it may not have a well-developed costa (hastula). Palmate leaves without a costa are placed in the coryphoide-lepidocaryoid clade (Moore and Uhl, 1982; Uhl et al., 1987). None of the several estipites (Romero, 1968; Arguijo, 1979, 1981) and fruits (Futey et al., 2012) known from the Salamanca Formation corresponds to palmate leaved taxa.

A separate specimen (MPEF-Pb-4154) from the CP quarry (not placed in a morphotype) that may be related to morphotype SA072, corresponds 
to a rachis fragment $(8 \mathrm{~cm}$ in length and $3.8 \mathrm{~cm}$ in width), preserving the rachis margin, which is smooth (unarmed) with small striations parallel to the long axis of the rachis.

\section{DISCUSSION}

The Salamanca and Peñas Coloradas formations from central Patagonia yield a well-dated time series of abundant, diverse, and exceptionally preserved Danian macrofloras from the Southern Hemisphere. Spanning three time slices, the oldest of these assemblages correlate to Chron C29n (early Danian, 65.58-64.86 Ma, Table 1), postdating the end-Cretaceous extinction by about one million years or less and offering insight into floral recovery following this catastrophic event. Unbiased collecting at these Danian sites yielded over 3,400 leaf compression fossils, dominated by angiosperms in both abundance (> 95\%) and richness (51 morphotypes here presented, vs. three conifer and five fern morphotypes or species (see Introduction). The angiosperm leaf dominance contrasts with the fossil wood assemblages from the same units that are dominated by gymnosperms (e.g., Petriella, 1972; Brea et al., 2005; Ruiz et al., 2020) and the high diversity of conifers and pteridophytes in the local palynofloras (Archangelsky, 1973, 1976a, 1976b; Petriela and Archangelsky, 1975; Clyde et al., 2014), indicating heterogeneity in source forest composition and the well-known, differing taphonomic pathways for leaf, wood, and pollen deposition (Behrensmeyer et al., 2000; Comer et al., 2015). The elevated overall diversity of leaves detailed here from about $51^{\circ} \mathrm{S}$ paleolatitude (Iglesias et al., 2007; Stiles et al., 2020) and their associated insect damage (Donovan et al., 2016,2018 ) is remarkable in light of the typically depauperate early Paleocene floras and insectdamage associations that are well known from similar absolute paleolatitudes (i.e., ca. $50^{\circ} \mathrm{N}$ ) in North America (i.e., North Dakota, Montana; e.g., Johnson, 2002; Iglesias et al., 2007; Donovan et al., 2014). However, there is increasing evidence of higher floral richness in some North American early Paleocene floras at lower paleolatitudes (e.g., Colorado, New Mexico; Johnson and Ellis, 2002; Flynn and Peppe, 2019; Lyson et al., 2019).

As would be expected in any comparable fossiliferous sequence, the composition and relative abundances of the compression floras are not completely uniform through time (Table 1). However, overall turnover among these floras is remarkably low, especially considering the variable environments of deposition within the ancient Sala- manca estuary and the switch to almost entirely continental environments during Peñas Coloradas Formation deposition (see Comer et al., 2015). Dividing the fossil quarries among the three paleomagnetic chrons sampled here $(\mathrm{C} 29 \mathrm{n}$, early Danian, 65.58-64.86 Ma; C28n, early Danian, 64.67-63.49 Ma; C27n, late Danian, 62.52-62.22 Ma; Table 1); 19 of the 51 (37\%) leaf morphotypes are present in all three time intervals. Of the 40 morphotypes present during C29n, 29 leaf types are found in $\mathrm{C} 28 \mathrm{n}(73 \%)$. Of the 32 morphotypes present in C28n, from estuarine deposits, 21 (66\%) are found in $\mathrm{C} 27 \mathrm{n}$, in fluvial deposits. At the local scale of a single valley of the Salamanca Formation exposures, 25 of 30 species $(83 \%)$ found at quarry PL1 (C29n) 'survive' at PL2 (C28n), which is derived from a more landward depositional environment in the estuary system than PL1 (see Comer et al., 2015). Moreover, the same two leaf types (morphotypes SA010, Lauraceae sp., and SA009 "Cissites" patagonica) are the most abundant throughout all these assemblages (Table 1). The exception to the pattern is the Rancho Grande flora of the Salamanca Formation (C29n; under separate study, see Geological Setting; Jud et al., 2017). That assemblage represents an inner shelf depositional setting (Comer, et al., 2015) and preserves a low-diversity flora having little compositional overlap with those reported here, showing that there was floristic variation associated with very broad environmental gradients and notably long transport distances.

Overall, whereas Danian plant diversity in Patagonia was high in comparison with many North American floras (per Iglesias et al., 2007), the homogeneous nature of the Patagonian floras through time and across facies constitutes a remarkable similarity to most of the North American record of this time interval, especially at comparable absolute paleolatitudes (e.g., Brown, 1962; Hickey, 1980; Johnson, 2002; Nichols and Johnson, 2008; see also prior discussion). Our results suggest that compositionally homogeneous regional floras, potentially dominated by generalist taxa, may have occupied extensive areas of the Earth for long stretches of time after the end-Cretaceous extinction. However, several paleobotanical sites from North America, as well as the Rancho Grande flora in the Salamanca Formation, show that much remains to be learned about how latitudinal, topographic, and environmental variation fostered heterogeneous patterns of early Paleocene floral recovery and diversity (Johnson and Ellis, 
2002; Ellis et al., 2003; Flynn and Peppe, 2019; Lyson et al., 2019).

The Danian floras presented here, while selfsimilar, appear to be quite distinct from other roughly coeval floras so far known from the Southern Hemisphere and appear to include many paleo-endemic taxa. We recognize very few angiosperm fossils that show close similarities to the Patagonian morphotypes presented here from latest Cretaceous to Paleocene assemblages of New Zealand and Antarctica (Pole, 1992, 1997; Dutra and Batten, 2000; Kennedy et al., 2002; Kennedy, 2003; Tosolini et al., 2013, 2020). Exceptions are the generally widespread leaf morphotypes that probably correspond to Nothofagaceae (here, morphotype SA004) and Lauraceae (SA010, SA046).

Within Patagonia, compression macrofloras from the latest Maastrichtian (67-66 Ma) portion of the Lefipán Formation in northwest Chubut (Barreda et al., 2012; Donovan et al., 2016, 2018; Wilf et al., 2017; Escapa et al., 2018; see also Martínez et al., 2018) are of considerable interest because they are very close to the Danian assemblages in time and space but separated from them by the terminal Cretaceous event. Quantitative comparisons and other analyses of the Lefipán and Salamanca-Las Flores floras are the topic of a related paper (Stiles et al., 2020).

Otherwise, the closest well-sampled points of comparison in Patagonia are the early Eocene (ca. $52 \mathrm{Ma}$ ), extremely diverse caldera-lake flora from Laguna del Hunco, also in northwest Chubut, and the early middle Eocene (ca. $47.8 \mathrm{Ma}$ ) Río Pichileufú flora from Río Negro Province (e.g., Berry, 1925a, 1938; Wilf et al., 2003, 2005; Wilf, 2012). Although more than 10 million years younger, the Eocene Laguna del Hunco and Pichileufu floras together include most of the plant clades so far identified from the Danian floras, including Agathis, Dacrycarpus, Arecaceae, Fabaceae, Lauraceae, Menispermaceae, Rhamnaceae, and diverse Cunoniaceae (compare Table 1; e.g., Wilf et al., 2005, 2012, 2014; Brea et al., 2008; Gandolfo and Hermsen, 2017; Jud et al., 2017, 2018a, 2018b; Escapa et al., 2018). These results, together with the apparent paleo-endemism of the Paleocene flora and the stem positions of Salamanca Formation fossils that have been phylogenetically ana- lyzed (Jud et al., 2017, 2018a; Escapa et al., 2018; Andruchow-Colombo et al., 2018) suggest that in situ diversification following the terminal Cretaceous event contributed significantly to the noted diversity of Eocene Patagonian floras. This idea raises interesting questions about when and where the trans-Antarctic floristic links from South America to Australia developed that characterize Eocene Gondwanan floras (e.g., Hill and Brodribb, 1999; Wilf et al., 2013; Kooyman et al., 2014; Barreda et al., 2020).

The richness of Paleocene Patagonian floras, along with the growing list of $\mathrm{K}-\mathrm{Pg}$ survivor plant and animal taxa (see discussions in Wilf et al., 2013, 2017) and the rapid Danian rebound of plant-insect associations (Donovan et al., 2016, 2018,2020 ), contributes to the emerging understanding of significant regional variation in ecosystem response to the terminal Cretaceous event and the role of Gondwana in the recovery phase.

\section{ACKNOWLEDGMENTS}

For generous support, we thank the National Science Foundation (grants DEB-1556666, DEB0345750, DEB-0919071, and EAR-1925755), the Consejo Nacional de Investigaciones Científicas y Técnicas (CONICET-Argentina), and the National Agency for Scientific and Technological Promotion (MINCYT-Argentina). Funding to E. Stiles came from Geological Society of America Student Research Grant No.12008-18 and a Mid-American Paleontological Society (MAPS) Outstanding Research Award. We thank I. Miller and M. Brea for reviews; M. Caffa, L. Canessa, B. Cariglino, R. Cúneo, M. Raigemborn, I. Escapa, C. González, K. Johnson, P. Puerta, S. Little, E. Ruigomez, M. Carvalho, M. Dawkins, A. Guillaume, and the late A. Zamuner for dedicated assistance in the field and laboratory; E. Romero and M. del C. Zamaloa for help with the UBA collections; J. Wingerath for assistance with Berry's type specimens at USNM; and for land access Secretaría de Cultura de la Provincia de Chubut, Panamerican Energy, and the Bochatey, de Galáz, Martínes, Porto, Salazar, Slápeliz, and Visser families. T. Dutra and T. Wang kindly provided the Portuguese and Chinese translations of the abstract, respectively. 


\section{REFERENCES}

Adanson, M. 1763. Familles des Plantes. Vincent, Paris. https://doi.org/10.5962/bhl.title.271

Ameghino, F. 1906. Les formations sédimentaires du Crétacé Superieur et du Tertiaire de Patagonie avec un paralléle entre leurs faunes mammalogiques et celles de l'ancien continent. Anales del Museo Nacional de Historia Natural de Buenos Aires, 15:1-568.

Andrews, H.C. 1809. The Botanist's Repository for New, and Rare Plants, v. 3-4. T. Bensley, London. https://doi.org/10.5962/bhl.title.51972

Andruchow-Colombo, A., Escapa, I.H., Carpenter, R.J., Hill, R.S., Iglesias, A., Arbazua, A.M., and Wilf, P. 2018. Oldest record of the scale-leaved clade of Podocarpaceae, early Paleocene of Patagonia, Argentina. Alcheringa, 43:127-145. https://doi.org/10.1080/03115518.2018.1517222

Archangelsky, S. 1973. Palinología del Paleoceno de Chubut. I. Descripciones sistemáticas. Ameghiniana, 10:339-399.

Archangelsky, S. 1976a. Palinología del Paleoceno de Chubut. II. Diagramas polínicos. Ameghiniana, 13:43-55.

Archangelsky, S. 1976b. Palinología del Paleoceno de Chubut. III. Análisis numérico. Ameghiniana, 13:169-184.

Arguijo, M.H. 1979. Palmoxylon bororoense n. sp. de la Formación Cerro Bororó (Paleoceno), Provincia de Chubut, República Argentina. Physis C, 38:87-96.

Arguijo, M.H. 1981. Palmoxylon vaterum n. sp. del Paleoceno (Daniano) de la Provincia de Chubut, República Argentina. Physis C, 39:49-59.

Arguijo, M.H. and Romero, E.J. 1981. Análisis bioestratigráfico de formaciones portadores de tafofloras Terciarias. VIII Congreso Geológico Argentino, San Luis, Actas, 4:691-717.

Ash, A., Ellis, B., Hickey, L.J., Johnson, K., Wilf, P., and Wing, S. 1999. Manual of Leaf Architecture: Morphological Description and Categorization of Dicotyledonous and NetVeined Monocotyledonous Angiosperms. Smithsonian Institution, Washington, D.C.

Askin, R.A. 1988. The palynological record across the Cretaceous/Tertiary transition on Seymour Island, Antarctica. Geological Society of America Memoirs, 169:155-162. https://doi.org/10.1130/mem169-p155

Awasthi, N. 1984. Studies on some carbonized woods from the Neyveli Lignite deposits, India. Geophytology, 14:82-95.

Barreda, V.D., Cúneo, N.R., Wilf, P., Currano, E.D., Scasso, R.A., and Brinkhuis, H. 2012. Cretaceous/Paleogene floral turnover in Patagonia: Drop in diversity, low extinction, and a Classopollis spike. PLoS One, 7:e52455. https://doi.org/10.1371/journal.pone.0052455

Barreda, V.D., Palazzesi, L., Pujana, R.R., Panti, C., Tapia, M.J., Fernández, D.A., and Noetinger, S. 2020. The Gondwanan heritage of the Eocene-Miocene Patagonian floras. Journal of South American Earth Sciences, 103022. https://doi.org/10.1016/j.jsames.2020.103022

Behrensmeyer, A.K., Kidwell, S.M., and Gastaldo, R.A. 2000. Taphonomy and paleobiology. Paleobiology, 26S:103-147. https://doi.org/10.1017/s0094837300026907

Bentham, G. and Hooker, J.D. 1862. Genera Plantarum ad Exemplaria Imprimis in Herbariis Kewensibus Servata Definita. A. Black. London. https://doi.org/10.5962/bhl.title.747

Berchtold, F. and Presl, J. 1820. O Přirozenosti Rostlin aneb. Rostlinář. Krala Wiljma Endersa, Prague.

Berg, O.K. 1854. Revisio Myrtacearum Americae, huc usque cognitarum s. Klotzschii, "Flora Americae aequinoctialis" exhibens Myrtaceas, auct. Linnaea 27:1-472.

Berry, E.W. 1925a. A Miocene flora from Patagonia. Johns Hopkins University Studies in Geology, 6:183-251.

Berry, E.W. 1925b. Fossil plants from the Tertiary of Patagonia and their significance. Proceedings of the National Academy of Sciences USA, 11:404-405. https://doi.org/10.1073/pnas.11.7.404

Berry, E.W. 1928. Tertiary fossil plants from the Argentine Republic. Proceedings of the U.S. National Museum, 73:1-27. https://doi.org/10.5479/si.00963801.73-2743.1

Berry, E.W. 1935a. A fossil Cochlospermum from northern Patagonia. Bulletin of the Torrey Botanical Club, 62:65-67. https://doi.org/10.2307/2480867 
Berry, E.W. 1935b. A preliminary contribution to the floras of the Whitemud and Ravenscrag formations. Canada Department of Mines, Geological Survey Memoirs, 182:1-107. https://doi.org/10.4095/100814

Berry, E.W. 1938. Tertiary flora from the Río Pichileufú, Argentina. Geological Society of America Special Paper, 12:1-149.

Berry, E.W. 1937a. A Paleocene flora from Patagonia. Johns Hopkins University Studies in Geology, 12:33-50.

Berry, E.W. 1937b. An Upper Cretaceous flora from Patagonia. Johns Hopkins University Studies in Geology, 12:11-31.

Blume, K.L.v. 1851. Museum botanicum Lugduno-Batavum, sive, Stirpium exoticarium novarum vel minus cognitarum ex vivis aut siccis brevis expositio et descriptio. Brill, Lyon. https://doi.org/10.5962/bhl.title.274

Bonaparte, J.F., Van Valen, L.M., and Kramartz, A. 1993. La fauna local de Punta Peligro, Paleoceno inferior, de la provincia del Chubut, Patagonia, Argentina. Evolutionary Monographs, 14:1-61.

Bradford, J.C. and Barnes, R.W. 2001. Phylogenetics and classification of Cunoniaceae (Oxalidales) using chloroplast DNA sequences and morphology. Systematic Botany, 26:354385.

Brea, M., Matheos, S.D., Raigemborn, M.S., Iglesias, A., Zucol, A.F., and Prámparo, M. 2007. Aspectos paleoecológicos y paleoambientales del Bosque Petrificado Ameghino (Daniano), Chubut, Argentina. 4th European Meeting on the Palaeontology and Stratigraphy of Latin America, Madrid. Cuadernos del Museo Geominero, 8:45-50.

Brea, M., Matheos, S.D., Raigemborn, M.S., Iglesias, A., Zucol, A.F., and Prámparo, M. 2011. Paleoecology and paleoenvironments of Podocarp trees in the Ameghino Petrified forest (Golfo San Jorge Basin, Patagonia, Argentina): Constraints for Early Paleogene paleoclimate. Geologica Acta, 9:13-28. https://doi.org/10.1344/105.000001647

Brea, M., Matheos, S., Zamuner, A., and Ganuza, D. 2005. Análisis de los anillos de crecimiento del bosque fósil de Víctor Szlápelis, Terciario Inferior del Chubut, Argentina. Ameghiniana, 42:407-418.

Brea, M., Zamuner, A.B., Matheos, S.D., Iglesias, A., and Zucol, A.F. 2008. Fossil wood of the Mimosoideae from the early Paleocene of Patagonia, Argentina. Alcheringa 32:427-441. https://doi.org/10.1080/03115510802417695

Brea, M. and Zucol, A.F. 2006. Leños fósiles de Boraginaceae de la Formación Peñas Coloradas (Paleoceno superior), Puerto Visser, Chubut, Argentina. Ameghiniana, 43:139146.

Brea, M., Zucol, A.F., Bargo, M.S., Fernicola, J.C., and Vizcaíno, S.F. 2018. First Miocene record of Akaniaceae in Patagonia (Argentina): a fossil wood from the early Miocene Santa Cruz formation and its palaeobiogeographical implications. Botanical Journal of the Linnean Society, 183:334-347. https://doi.org/10.1093/botlinnean/bow014

Brongniart, A.T. 1826. Memoire sur la famille des Rhamnées. Didot le Jeune, Paris.

Brown, R. 1814. General Remarks, Geographical and Systematical, on the Botany of Terra Australis. In Flinders, M. A Voyage to Terra Australis 2, Appendix 3. W. Bulmer, London. https://doi.org/10.5962/bhl.title.50709

Brown, R.W. 1962. Paleocene flora of the Rocky Mountains and Great Plains. Geological Survey Professional Paper 375, United States Government Printing Office, Washington. https://doi.org/10.3133/pp375

Carvalho, M.R., Herrera, F.A., Jaramillo, C.A., Wing, S.L., and Callejas, R. 2011. Paleocene Malvaceae from Northern South America and their biogeographical implications. American Journal of Botany, 98:1337-1355. https://doi.org/10.3732/ajb.1000539

Clyde, W.C., Wilf, P., Iglesias, A., Slingerland, R.L., Barnum, T., Bijl, P.K., Bralower, T.J., Brinkhuis, H., Comer, E.E., Huber, B.T., Ibañez-Mejia, M., Jicha, B.R., Krause, J.M., Schueth, J.D., Singer, B.S., Raigemborn, M.S., Schmitz, M.D., Sluijs, A., and Zamaloa, M.C. 2014. New age constraints for the Salamanca Formation and lower Río Chico Group in the western San Jorge Basin, Patagonia, Argentina: Implications for Cretaceous/Paleogene extinction recovery and land mammal age correlations. Geological Society of America Bulletin, 126:289-306. https://doi.org/10.1130/b30915.1 
Comer, E.E., Slingerland R.L., Krause J.M., Iglesias A., Clyde, W.C., Raigemborn, M.S., and Wilf, P. 2015. Sedimentary facies and depositional environments of diverse early Paleocene floras, north-central San Jorge Basin, Patagonia, Argentina. Palaios, 30:553-573. https://doi.org/10.2110/palo.2014.064

de Laubenfels, D.J. 1969. A revision of the Malesian and Pacific rainforest conifers, I. Podocarpaceae in part. Journal of the Arnold Arboretum, 50:274-369. https://doi.org/10.5962/bhl.part.24691

Donovan, M.P., Wilf, P., Labandeira, C.C., Johnson, K.R., and Peppe, D.J. 2014. Novel insect leaf-mining after the end-Cretaceous extinction and the demise of Cretaceous leaf miners, Great Plains, USA. PLoS One, 9:e103542. https://doi.org/10.1371/journal.pone.0103542

Donovan, M.P., Iglesias, A., Wilf, P., Labandeira, C.C., and Cúneo, N.R. 2016. Rapid recovery of Patagonian plant-insect associations after the end-Cretaceous extinction. Nature Ecology \& Evolution, 1:0012. https://doi.org/10.1038/s41559-016-0012

Donovan, M.P., Iglesias, A., Wilf, P., Labandeira, C.C., and Cúneo, N.R. 2018. Diverse plantinsect associations from the latest Cretaceous and early Paleocene of Patagonia, Argentina. Ameghiniana, 55:330-338. https://doi.org/10.5710/amgh.15.02.2018.3181

Donovan, M.P., Wilf, P., Iglesias, A., Cúneo, N.R., and Labandeira, C.C. 2020. Persistent biotic interactions of a Gondwanan conifer from Cretaceous Patagonia to modern Malesia. Communications Biology 3:708. https://doi.org/10.1038/s42003-020-01428-9

Dunn, R.E., Madden, R.H., Kohn, M.J., Schmitz, M.D., Strömberg, C.A.E., Carlini, A.A., Ré, G.H., and Crowley, J. 2013. A new chronology for middle Eocene-early Miocene South American Land Mammal Ages. Geological Society of America Bulletin, 125:539-555. https://doi.org/10.1130/b30660.1

Dusén, P. 1907. Über die Tertiäre Flora der Magellansländer, p.87-108. In Nordenskjöld, O. (ed.), Wissenschaftliche Ergebnisse der Schwedischen Expedition nach den Magellansländern 1895-1897, Band I. Norstedt and Söner, Stockholm. https://doi.org/10.5962/bhl.title.111414

Dusén, P. 1908. Über Die Tertiare Flora der Seymour Insel, p. 1-27. In Nordenskjöld, O. (ed.), Wissenschaftliche Ergebnisse der Schwedischen Südpolar-Expedition 1901-1903, Geologie und Paläontologie 3. Institut des Generalstaes, Stockholm. https://doi.org/10.5962/ bhl.title. 111414

Dutra, T.L. and Batten, T.J. 2000. Upper Cretaceous floras of King George Island, West Antarctica, and their palaeoenvironmental and phytogeographic implications. Cretaceous Research, 21:181-209. https://doi.org/10.1006/cres.2000.0221

Ellis, B., Daly, D.C., Hickey, L.J., Johnson, K.R., Mitchell, J.D., Wilf, P., and Wing, S.L. 2009. Manual of Leaf Architecture. Cornell University Press, New York.

Ellis, B., Johnson, K.R., and Dunn, R.E. 2003. Evidence for an in situ early Paleocene rainforest from Castle Rock, Colorado. Rocky Mountain Geology, 38:73-100. https://doi.org/10.2113/gsrocky.38.1.173

Engelhardt, H. 1891. Ueber Tertiärpflanzen von Chile. Abhandlungen der Senckenbergischen Naturforschenden Gesellschaft, 16:629-692.

Escapa, I.H., Iglesias, I., Wilf, P., Catalano, S.A., Caraballo-Ortiz, M.A., and Cúneo, N.R. 2018. Agathis trees of Patagonia's Cretaceous-Paleogene death landscapes and their evolutionary significance. American Journal of Botany, 105:1345-1368. https://doi.org/10.1002/ajb2.1127

Espinosa, M.R. 1926. Nothofagus alessandrii Espinosa. Revista Chilena de Historia Natural, 30:268.

Feild, T.S., Brodribb, T.J., Iglesias, A., Chatelet, D.S., Baresch, A., Upchurch, G.R. Jr., Gomez, B., Mohr, B.A.R., Coiffard, C., Kvaček, J., and Jaramillo, C.A. 2011. Fossil evidence for Cretaceous escalation in angiosperm leaf vein evolution. Proceedings of the National Academy of Sciences of the United States of America, 108:8363-8366. https://doi.org/ 10.1073/pnas.1014456108

Ferrow, E., Vajda, V., Koch, C.B., Peucker-Ehrenbrink, B., and Willumsen, P.S. 2011. Multiproxy analysis of a new terrestrial and a marine Cretaceous-Paleogene (K-Pg) boundary site from New Zealand. Geochimica et Cosmochimica Acta, 75:657-672. https://doi.org/10.1016/j.gca.2010.10.016

Feruglio, E. 1949. Descripción geológica de la Patagonia, Tomo I. Ministerio de Industria y Comercio de la Nación, Dirección General de Yacimientos Petroliferos Fiscales, Coni, Buenos Aires. 
Fischer, T.C., Butzmann, R., Meller, B., Rattei, T., Newman, M., and Hölscher, D. 2009. The morphology, systematic position and inferred biology of Spirematospermum - An extinct genus of Zingiberales. Review of Palaeobotany and Palynology, 157:391-426. https://doi.org/10.1016/j.revpalbo.2009.06.010

Flynn, A.G. and Peppe, D.J. 2019. Early Paleocene tropical forest from the Ojo Alamo Sandstone, San Juan Basin, New Mexico, USA. Paleobiology, 45:612-635. https://doi.org/10.1017/pab.2019.24

Futey, M.K., Gandolfo, M.A., Zamaloa, M.C., Cúneo, N.R., and Cladera, G. 2012. Arecaceae fossil fruits from the Paleocene of Patagonia, Argentina. The Botanical Review, 78:205-234. https://doi.org/10.1007/s12229-012-9100-9

Gandolfo, M.A., Dibbern, M.C., and Romero, E.J. 1988. Akania patagonica n. sp. and additional material on Akania americana Romero \& Hickey (Akaniaceae), from Paleocene sediments of Patagonia. Bulletin of the Torrey Botanical Club, 115:83-88. https://doi.org/10.2307/2996138

Gandolfo, M.A. and Hermsen, E.J. 2017. Ceratopetalum (Cunoniaceae) fruits of Australasian affinity from the early Eocene Laguna del Hunco flora, Patagonia, Argentina. Annals of Botany, 119:507-516. https://doi.org/10.1093/aob/mcw283

Gandolfo, M.A., Hermsen, E.J., Zamaloa, M.C., Nixon, K.C., González, C.C., Wilf, P., Cúneo, N.R., and Johnson, K.R. 2011. Oldest known Eucalyptus macrofossils are from South America, PloS One, 6:e21084. https://doi.org/10.1371/journal.pone.0021084

Gibbs, L.S. 1917. Dutch N.W. New Guinea: A Contribution to the Phytogeography and Flora of the Arfak Mountains, Taylor and Francis, London. https://doi.org/10.5962/bhl.title.31591

González, C.C. 2008. Revisión Taxonómica de las Familias de Angiospermas Dominantes de la "Flora del Hunco" (Eoceno Temprano), Chubut, Argentina. Unpublished PhD Thesis. Buenos Aires University, Buenos Aires, Argentina.

Gradstein, F.M., Ogg, J.G., Schmitz, M.D., and Ogg, G.M. (eds.) 2012. The Geologic Time Scale 2012. Elsevier, Amsterdam. https://doi.org/10.1016/c2011-1-08249-8

Gray, A. 1854. United States Exploring Expedition During the Years 1838, 1839, 1840, 1841, 1842 Under the Command of Charles Wilkes, U.S.N. vol. XV. Botany. Phanerogamia. C. Sherman, Philadelphia. https://doi.org/10.5962/bhl.title.61410

Gray, S.F. 1822. A Natural Arrangement of British Plants, according to their relations to each other as pointed out by Jussieu, De Candolle, Brown, \&c. Baldwin, Cradock and Joy, London. https://doi.org/10.5962/bhl.title.43804

Green, W.A., Little, S.A., Price, C.A., Wing, S.L., Smith, S.Y., Kotrc, B., and Doria. G. 2014. Reading the leaves: A comparison of leaf rank and automated areole measurement for quantifying aspects of leaf venation. Applications in Plant Sciences, 2:1-1400006. https://doi.org/10.3732/apps.1400006

Grisebach, A. 1854. Grundriss der Systematischen Botanik für Akademische Vorlesungen Entworfen. Dieterischen Buchhandlung, Göttingen.

Hermsen, J.H., Jud, N.A., De Benedetti, F., and Gandolfo, M.A. 2019. Azolla sporophytes and spores from the Late Cretaceous and Paleocene of Patagonia, Argentina. International Journal of Plant Sciences, 180:737-754. https://doi.org/10.1086/704377

Hickey, L.J. 1971. Evolutionary significance of leaf architectural features in the woody dicots. (Abstract) American Journal of Botany, 58:469.

Hickey, L.J. 1973. Classification of the architecture of dicotyledonous leaves. American Journal of Botany, 60:17-33. https://doi.org/10.1002/j.1537-2197.1973.tb10192.x

Hickey, L.J. 1979. A revised classification of the architecture of dicotyledonous leaves, p. 25-39. In Metcalfe, C.R. and Chalk, L. (eds.), Anatomy of the Dicotyledons, 2nd edition. Oxford, Clarendon.

Hickey, L.J. 1980. Paleocene stratigraphy and flora of the Clark's Fork Basin, p. 33-49. In Gingerich, P.D. (ed.), Early Cenozoic Paleontology and Stratigraphy of the Bighorn Basin, Wyoming: 1880-1980, University of Michigan Papers on Paleontology 24, Ann Arbor, Michigan.

Hickey, L.J. and Peterson, R.K., 1978. Zingiberopsis, a fossil genus of the ginger family from Late Cretaceous to early Eocene sediments of Western Interior North America. Canadian Journal of Botany, 56:1136-1152. https://doi.org/10.1139/b78-128

Hickey, L.J. and Wolfe, J.A. 1975. The bases of angiosperm phylogeny: vegetative morphology. Annals of the Missouri Botanical Garden, 62:538-589. https://doi.org/10.2307/2395267

Hill, R.S. and Brodribb, T.J. 1999. Southern conifers in time and space. Australian Journal of Botany, 47:639-696. https://doi.org/10.1071/BT98093 
Hinojosa, L.F., Pérez, F., Gaxiola, A., and Sandoval, I. 2011. Historical and phylogenetic constraints on the incidence of entire leaf margins: insights from a new South American model. Global Ecology and Biogeography, 20:380-390.

https://doi.org/10.1111/j.1466-8238.2010.00595.x

Hitchcock, E. 1858. Ichnology of New England. A Report on the Sandstone of the Connecticut Valley, Especially Its Fossil Footmarks. W. White, Boston.

Hünicken, M.A. 1967. Flora Terciaria de los estratos de Río Turbio, Santa Cruz: niveles plantíferos del arroyo Santa Flavia. Revista de la Facultad de Ciencias Exactas, Físicas y Naturales de la Universidad de Córdoba, Serie Ciencias Naturales, 27:139-227.

Hünicken, M.A. 1995. Floras Cretácicas y Terciarias. Actas de la Academia Nacional de Ciencias Córdoba, 11:199-220.

Iglesias, A. 2007. Estudio Paleobotánico, Paleoecológico y Paleoambiental en Secuencias de la Formación Salamanca, del Paleoceno Inferior en el Sur de la Provincia de Chubut, Patagonia, Argentina. Unpublished Ph.D. Thesis, La Plata University, Buenos Aires, Argentina.

Iglesias, A., Wilf, P., Johnson, K.R., Zamuner, A.B., Cúneo, N.R., Matheos, S.D., and Singer, B.S. 2007. A Paleocene lowland macroflora from Patagonia reveals significantly greater richness than North American analogs. Geology, 35:947-950. https://doi.org/10.1130/G23889A.1

Johnson, K.R. 2002. Megaflora of the Hell Creek and lower Fort Union Formations in the western Dakotas: vegetational response to climate change, the Cretaceous-Tertiary boundary event, and rapid marine transgression. Geological Society of America Special Paper, 361:329-391. https://doi.org/10.1130/0-8137-2361-2.329

Johnson, K.R. and Ellis, B. 2002. A tropical rainforest in Colorado 1.4 million years after the Cretaceous/Tertiary boundary. Science, 296:2379-2383. https://doi.org/10.1126/science.1072102

Johnson, K.R., Nichols, D.J., Attrep, M. Jr., and Orth, C.J. 1989. High-resolution leaf-fossil record spanning the Cretaceous/Tertiary boundary. Nature, 340:708-711. https://doi.org/ 10.1038/340708a0

Jones, J.H., Manchester, S.R., and Dilcher, D.L. 1988. Dryophyllum Debey ex Saporta, juglandaceous not fagaceous. Review of Palaeobotany and Palynology, 56:205-211. https://doi.org/10.1016/0034-6667(88)90059-0

Jud, N.A., Gandolfo, M.A., Iglesias, A., and Wilf, P. 2017. Flowering after disaster: Early Danian buckthorn (Rhamnaceae) flowers and leaves from Patagonia. PLoS ONE, 12:e0176164. https://doi.org/10.1371/journal.pone.0176164

Jud, N.A., Gandolfo, M.A., Iglesias, A., and Wilf, P. 2018a. Fossil flowers from the early Palaeocene of Patagonia, Argentina, with affinity to Schizomerieae (Cunoniaceae). Annals of Botany, 121:431-442. https://doi.org/10.1093/aob/mcx173

Jud, N.A., Iglesias, A., Wilf, P., and Gandolfo, M.A. 2018b. Fossil moonseeds from the Paleogene of West Gondwana (Patagonia, Argentina). American Journal of Botany, 105:927942. https://doi.org/10.1002/ajb2.1092

Jussieu, A.L.de. 1789. Genera Plantarum. Herissant \& Barrois, Paris. https://doi.org/10.5962/bhl.title.284

Karsten, H. 1856. Deutsche Flora. Pharmaceutisch-medicinische Botanik. J.M. Spaeth, Berlin. https://doi.org/10.5962/bhl.title.12026

Keating, R.C. 2002. Leaf anatomical characters and their value in understanding morphoclines in the Araceae. The Botanical Review, 68:510-523.

Kennedy, E.M. 2003. Late Cretaceous and Paleocene terrestrial climates of New Zealand: leaf fossil evidence from South Island assemblages. New Zealand Journal of Geology and Geophysics, 46:295-306. https://doi.org/10.1080/00288306.2003.9515010

Kennedy, E.M., Spicer, R.A., and Rees, P.M. 2002. Quantitative palaeoclimate estimates from late Cretaceous and Paleocene leaf floras in the northwest of the South Island, New Zealand. Palaeogeography, Palaeoclimatology, Palaeoecology, 184:321-345. https://doi.org/10.1016/s0031-0182(02)00261-4

Kooyman, R.M., Wilf, P., Barreda, V.D., Carpenter, R.J., Jordan, G.J., Sniderman, J.M.K., Allen, A., Brodribb, T.J., Crayn, D., Feild, T.S. Laffan, S.W., Lusk, C.H., Rossetto, M., and Weston, P.H. 2014. Paleo-Antarctic rainforest into the modern Old World tropics: The rich past and threatened future of the "southern wet forest surviors". American Journal of Botany, 101:2121-2135. https://doi.org/10.3732/ajb.1400340 
Krause, J.M., Clyde, W.C., Ibañez-Mejía, M., Schmitz, M.D., Barnum, T., Bellosi, E.S., and Wilf, P. 2017. New age constraints for early Paleogene strata of central Patagonia, Argentina: implications for the timing of South America land mammal ages. Geological Society of America Bulletin, 129:886-903. https://doi.org/10.1130/b31561.1

Kunth, C.S. 1822. Synopsis Plantarum Quas in Itinere ad Plagam Aequinoctialem Orbis Novi Collegerunt Humboldt et Bonpland. Levraut, Paris.

Kuprianova, A.L. 1962. Palinological data and the systematics of the Fagales and Urticales. In Sladkov, A.N. (ed.), For the First International Conference on Palinology: Reports of Soviet Palinologists (extended theses). U.S.S.R. Academy of Sciences, Moscow.

Labandeira, C.C., Wilf, P., Johnson, K.R., and Marsh, F. 2007. Guide to Insect (and Other) Damage Types on Compressed Plant Fossils. Version 3.0. Smithsonian Institution, Washington D.C.

Lamarck, J.B.P.A.M.C. 1783. Encyclopédie Méthodique, Botanique, I. Pancoucke, Paris. https://doi.org/10.5962/bhl.title.824

Lamarck, J.B.P.A.M.C. 1789. Encyclopédie Méthodique, Botanique, III. Pancoucke, Paris. https://doi.org/10.5962/bhl.title.824

Legarreta, L. and Uliana, M.A. 1994. Asociaciones de fósiles y hiatos en el SupracretácicoNeogeno de la Patagonia: una perspectiva estratigráfico-secuencial. Ameghiniana, 31:257281.

Lindley, J. 1836. A Natural System of Botany; or, A systematic View of the Organization, Natural Affinities, and Geographical Distribution of the Whole Vegetable Kingdom: Together with the Uses of the Most Important Species in Medicine, the Arts, and Rural or Domestic Economy. Longman, Rees, Orme, Brown, Green, and Longman, London. https://doi.org/10.5962/ bhl.title.130142

Linnaeus, C. 1753. Species Plantarum. Laurentii Salvii, Stockholm.

Little, S.A., Green, W.A., Wing, S.L., and Wilf, P. 2014. Reinvestigation of leaf rank, an underappreciated component of Leo Hickey's legacy. Bulletin of the Peabody Museum of Natural History, 55:79-87. https://doi.org/10.3374/014.055.0202

Lyson, T.R., Miller, I.M., Bercovici, A.D., Weissemburger, K., Fuentes, A.J., Clyde, W.C., Hagadorn, J.W., Butrim, M.J., Johnson, K.R., Fleming, R.F., Barclay, R.S., Maccracken, S.A., Lloyd, B., Wilson, G.P., Krause, D.W., and Chester, S.G.B. 2019. Exceptional continental record of biotic recovery after the Cretaceous-Paleogene mass extinction. Science, 366:977983. https://doi.org/10.1126/science.aay2268

Mabberley, D.J. 1989. The Plant-Book. A Portable Dictionary of the Higher Plants. Cambridge University Press, Cambridge.

Macphail, M.K. 1994. Impact of the K/T event on the southeast Australian flora and vegetation: mass extinction, niche disruption or nil? Palaeoaustral, 1:9-13.

Mann, G. and Wendland, H. 1864. On the Palms of Western Tropical Africa. Transactions of the Linnean Society of London, 24:421-439. https://doi.org/10.1111/j.1096-3642.1863.tb00165.x

Martínez, C., Gandolfo, M.A., and Cúneo, N.R. 2018. Angiosperm leaves and cuticles from the uppermost Cretaceous of Patagonia, biogeographic implications and atmospheric paleo- $\mathrm{CO}_{2}$ estimates. Cretaceous Research, 89:107-118. https://doi.org/10.1016/j.cretres.2018.03.015

Maund, B. 1840. The Botanist: containing accurately coloured figures of tender and hardy ornamental plants; with descriptions, scientific and popular; intended to convey both moral and intellectual gratification. R. Groombridge, W. White and Co., M. Calignani, Huaman and Co., and Jackson, London.

Mayo, S.J., Bogner, J., and Boyce, P.C. 1997. The Genera of Araceae. Royal Botanic Gardens, Kew, UK.

Menéndez, C.A. 1959. Flora Cretácica de la Serie de Castillo, al S del Cerro Cachetaman, Chubut. Revista de la Asociación Geológica Argentina, 14:219-238.

Miller, P. 1754. The Gardeners Dictionary. J. and J. Rivington, London. https://doi.org/10.5962/bhl.title.79061

Moore, H.E. and Uhl, N.W. 1982. Major trends of evolution in palms. The Botanical Review, 48:169. https://doi.org/10.1007/bf02860535

Mueller, F. 1858. Fragmenta Phytographiae Australiiae. Auctoritate Gubernamentale, Coloniae Victoriae, Melburne.

Nathorst, A.G. 1888. Zur Fossilen Flora Japan's. Palaeontologische Abhandlungen, 4:197-250.

Nichols, D.J. and Johnson, K.R. 2008. Plants and the K-T Boundary. Cambridge University Press, Cambridge. 
Oliver, D. 1881. Icones Plantarum or Figures, With Brief Descriptive Characters and Remarks, of New or Rare Plants, Selected From the Author's Herbarium, volume IV, page 27. In Hooker, W.J. (ed.), Longman, Rees, Orme, Brown, Green \& Longman, London.

Pampanini, R. 1905. Le Cunoniacee degli Erbari di Firenze e di Ginevra. Annali di Botanica, 2:43-106

Pascual, R., Goin, F.J., Balarino, L., and Udrizar Sauthier, D.E. 2002. New data on the Paleocene monotreme Monotrematum sudamericanum, and the convergent evolution of triangulate molars. Acta Palaeontologica Polonica, 47:487-492.

Peppe, D.J., Royer, D.L., Cariglino, B., Oliver, S.Y., Newman, S., Leight, E., Enikolopov G., Fernandez-Burgos, M., Herrera, F., Adams, J.M., Correa, E., Currano, E.D., Erickson, J.M., Hinojosa, L.F., Hoganson, J.W., Iglesias, A., Jaramillo, C.A., Johnson, K.R., Jordan, G.J., Kraft, N.J.B., Lovelock, E.C., Lusk, C.H., Niinemets, Ü., Peñuelas, J., Rapson, G., Wing, S.L., and Wright, I.J. 2011. Sensitivity of leaf size and shape to climate: global patterns and paleoclimatic applications. New Phytologist, 190:724-739. https://doi.org/10.1111/j.1469-8137.2010.03615.x

Perleb, K.J. 1818. Versuch über die Arzneikräfte der Pflanzen verglichen mit den äusseren Formen und der natürlichen Klasseneintheilung derselben. Sauerländer, Aarau.

Petriella, B. 1972. Estudio de maderas petrificadas del Terciario inferior del área central de Chubut (Cerro Bororó). Revista del Museo de La Plata, 6:159-254.

Petriella, B. and Archangelsky, S. 1975. Vegetación y ambiente en el Paleoceno de Chubut. I Congreso Argentino de Paleontología y Bioestratigrafía, Tucumán, Actas, 2:257-270.

Pole, M. 1992. Cretaceous macrofloras of eastern Otago, New Zealand: Angiosperms. Australian Journal of Botany, 40:169-206. https://doi.org/10.1071/bt9920169

Pole, M. 1997. Paleocene plant macrofossils from Kakahu, South Canterbury, New Zealand. Journal of the Royal Society of New Zealand, 27:371-400. https://doi.org/10.1080/03014223.1997.9517544

Pole, M. and Vajda, V. 2009. A new terrestrial Cretaceous-Paleogene site in New Zealand turnover in macroflora confirmed by palynology. Cretaceous Research, 30:917-938. https://doi.org/10.1016/j.cretres.2009.02.007

Quiroga, M.P., Mathiasen, P., Iglesias, A., Mill, R.R., and Premoli, A.C. 2016. Molecular and fossil evidence disentangle the biogeographical history of Podocarpus, a key genus in plant geography. Journal of Biogeography, 43:372-383. https://doi.org/10.1111/jbi.12630

Ragonese, A.M. 1980. Leños fósiles de dicotiledóneas del Paleoceno de Patagonia, Argentina. I. Myrceugenia chubutense n. sp. (Myrtaceae). Ameghiniana, 17:297-311.

Raigemborn, M.S. 2005. Procedencia de las areniscas de la Formación Peñas Coloradas (Paleoceno Superior) en la zona costera al Norte de Comodoro Rivadavia, Cuenca del Golfo San Jorge (Chubut, Argentina). XVI Congreso Argentino de Geología, Buenos Aires, Argentina, Actas, 3:103-110.

Raigemborn, M.S. 2006. Análisis composicional y procedencia de la Formación Peñas Coloradas, Grupo Río Chico (Paleoceno superior-Eoceno?), en la región oriental de la Cuenca del Golfo San Jorge, Chubut, Argentina. Latin American Journal of Sedimentology and Basin Analysis, 13:119-133.

Raigemborn, M.S., Brea, M., Zucol, A., and Matheos, S.D. 2009. Early Paleogene climate at mid latitude in South America: Mineralogical and paleobotanical proxies from continental sequences in Golfo San Jorge basin (Patagonia, Argentina). Geologica Acta, 7:125-145. https://doi.org/10.1344/105.000000269

Raigemborn, M.S., Gómez-Peral, L.E., Krause, J.M., and Matheos, S.D. 2014. Controls on clay minerals assemblages in an early paleogene nonmarine succession: Implications for the volcanic and paleoclimatic record of extra-andean Patagonia, Argentina. Journal of South American Earth Sciences, 52:1-23. https://doi.org/10.1016/j.jsames.2014.02.001

Read, R.W. and Hickey, L.J. 1972. A revised classification of fossil palm and palm-like leaves. Taxon, 21:129-137. https://doi.org/10.2307/1219237

Richardson, J.E., Fay, M.F., Cronk, Q.C.B., and Chase, M.W. 2000. A revision of the tribal classification of Rhamnaceae. Kew Bulletin, 55: 311-340. https://doi.org/10.2307/4115645

Romero, E.J. 1968. Palmoxylon patagonicum n. sp., del Terciario Inferior de la Provincia de Chubut, Argentina. Ameghiniana, 5:417-432.

Romero, E.J. and Dibbern, M. 1985. A review of the species described as Fagus and Nothofagus by Dusén. Palaeontographica, Abteilung B-Paläophytologie, 197:123-137. 
Romero, E.J. and Hickey, L.J. 1976. A fossil leaf of Akaniaceae from Paleocene beds in Argentina. Bulletin of the Torrey Botanical Club, 103:126-131. https://doi.org/10.2307/2484888

Ruiz, D.P., Brea, M., Raigemborn, M.S., and Matheos S.D. 2017. Conifer woods from the Salamanca Formation (early Paleocene), Central Patagonia, Argentina: Paleoenvironmental implications. Journal of South American Earth Sciences, 76:427-445. https://doi.org/10.1016/j.jsames.2017.04.006

Ruiz, D.P., Raigemborn, M.S., Brea, M., and Pujana, R.R. 2020. Paleocene Las Violetas forest: Wood anatomy and paleoclimatology. Journal of South American Earth Sciences, 98:102414. https://doi.org/10.1016/j.jsames.2019.102414

Ruiz, H. and Pavón, J. 1797. Florae Peruvianae, et Chilensis Prodromus sieve novorum generum plantarum Peruvianarum, et Chilensium descriptiones, et icones. Paleariniano, Rome. https://doi.org/10.5962/bhl.title.11759

Salisbury, R.A. 1805. Description of the natural order of Nymphaeaceae. Annals of Botany, 2:6976.

Salisbury, R.A. 1807. The characters of several genera in the natural order of Coniferae: with remarks on their stigmata, and cotyledons. Transactions of the Linnean Society of London, 8:308-317. https://doi.org/10.1111/j.1096-3642.1807.tb00316.x

Saporta, G. de. 1862. Prodrome d'une flora fossile des travertins anciens de Sézanne. Mémoires de la Société Géologique de France, 8:289-436.

Schlechter, R. 1915. Die Cunoniaceae Papuasiens. Botanische Jahrbücher für Systematik, Pflanzengeschichte und Pflanzengeographie, 52:139-166.

Smith, A.C. 1793. A Specimen of the Botany of New Holland. J. Davis, London.

Stapf, O. 1912. Akaniaceae: A new family of Sapindales. Bulletin of Miscellaneous Information, Royal Botanical Gardens, Kew, 9:378-380. https://doi.org/10.2307/4104555

Steinthorsdottir, M., Vajda, V., and Pole, M. 2016. Global trends of $\mathrm{pCO}_{2}$ across the CretaceousPaleogene boundary supported by the first Southern Hemisphere stomatal proxy-based $\mathrm{pCO}_{2}$ reconstruction. Palaeogeography, Palaeoclimatology, Palaeoecology, 464:143-152. https://doi.org/10.1016/j.palaeo.2016.04.033

Sterli, J. and de la Fuente, M.S. 2012. New evidence from the Palaeocene of Patagonia (Argentina) on the evolution and palaeo-biogeography of Meiolaniformes (Testudinata, new taxon name). Journal of Systematic Palaeontology, 11:835-852. https://doi.org/10.1080/14772019.2012.708674

Stiles, E., Wilf, P., Iglesias, A., Gandolfo, M.A., and Cúneo, N.R. 2020. Cretaceous-Paleogene plant extinction and recovery in Patagonia. Paleobiology, 46:445-469. https://doi.org/10.1017/pab.2020.45

Sylwan, C.A. 2001. Geology of the Golfo San Jorge Basin, Argentina. Journal of Iberian Geology, 27:123-157.

Tambussi, C.P. and Degrange, F.J. 2013. The Paleogene birds of South America, p. 29-47. In Tambussi, C.P. and Degrange, F.J. (eds.), South American and Antarctic Continental Cenozoic Birds. Springer, Dordrecht, Netherlands. https://doi.org/10.1007/978-94-007-5467-6_5

Tanai, T. 1986. Phytogeographic and phylogenetic history of the genus Nothofagus BI. (Fagaceae) in the Southern Hemisphere. Journal of the Faculty of Sciences, Hokkaido University, Series 4, 21:505-582.

Tosolini A-M.P., Cantrill, D.J., and Francis, J.E. 2013. Paleocene flora from Seymour Island, Antarctica: revision of Dusén's (1908) angiosperm taxa. Alcheringa, 37:366-391. https://doi.org/10.1080/03115518.2013.764698

Tosolini A-M.P., Cantrill, D.J., and Francis, J.E. 2020. Paleocene high-latitude leaf flora of Antarctica Part 1: entire-margined angiosperms. Review of Palaeobotany and Palynology, 104317. https://doi.org/10.1016/j.revpalbo.2020.104317

Thouars, L.M.A.d.P. 1806. Genera Nova Madagascariensia secundum methodum lussiaenam disposita. Thouars, L.M.A.d.P. France.

Traverso, N.E. 1951. La Flora Fósil de El Mirador (Chubut) Conservada en el Museo de la Plata. Unpublished Ph.D. Thesis, La Plata University, La Plata, Buenos Aires, Argentina.

Troncoso, A.A. 1992. La tafoflora Terciaria de Quinamávida (VII Región, Chile). Boletín del Museo Nacional de Historia Natural de Chile, 43:155-178. 
Troncoso, A., Suárez, M., De la Cruz, R., and Palma-Heldt, S. 2002. Paleoflora de la Formación Ligorio Márquez (XI Región, Chile) en su localidad tipo: sistemática, edad e implicancias paleoclimáticas. Revista Geológica de Chile, 29:113-135. https://doi.org/10.4067/s0716-02082002000100007

Upchurch, G.R. and Dilcher, D.L. 1990. Cenomanian Angiosperm Leaf Megafossils, Dakota Formation, Rose Creek Locality, Jefferson County, Southeastern Nebraska. United States Geological Survey Bulletin, Denver, Colorado. https://doi.org/10.3133/b1915

Uhl, N.W., Moore, H.E., Dransfield, J., and Sheehan, M.R. 1987. Genera Palmarum. A Classification of Palms Based on the Work of Harold E. Moore, jr. The L.H. Bailey Hortorum and The International Palm Society, Lawrence, Kansas.

Vajda, V. and McLoughlin, S. 2006. Extinction and recovery patterns of the vegetation across the Cretaceous-Palaeogene boundary - a tool for unravelling the causes of the end-Permian mass-extinction. Review of Palaeobotany and Palynology, 144:99-112. https://doi.org/10.1016/j.revpalbo.2005.09.007

Vajda, V. and Raine, J.I. 2003. Pollen and spores in marine Cretaceous/Tertiary boundary sediments at mid-Waipara River, North Canterbury, New Zealand. New Zealand Journal of Geology and Geophysics, 46:255-273. https://doi.org/10.1080/00288306.2003.9515008

Vajda, V., Raine, J.I., and Hollis, C.J. 2001. Indication of global deforestation at the CretaceousTertiary boundary by New Zealand fern spike. Science, 294:1700-1702. https://doi.org/10.1126/science.1064706

Vieillot, L.J.P. 1818. Ornithologie. Lanoe, Paris.

Wilf, P. 2012. Rainforest conifers of Eocene Patagonia: Attached cones and foliage of the extant Southeast Asian and Australasian genus Dacrycarpus (Podocarpaceae). American Journal of Botany, 99:562-584. https://doi.org/10.3732/ajb.1100367

Wilf, P., Cúneo, N.R., Johnson, K.R., Hicks, J.F., Wing, S.L., and Obradovich, J.D. 2003. High plant diversity in Eocene South America: Evidence from Patagonia. Science, 300:122-125. https://doi.org/10.1126/science.1080475

Wilf, P., Johnson, K.R., Cúneo, N.R., Smith, M.E., Singer, B.S., and Gandolfo, M.A. 2005. Eocene plant diversity at Laguna del Hunco and Río Pichileufú, Patagonia, Argentina. The American Naturalist, 165:634-650. https://doi.org/10.1086/430055

Wilf, P., Cúneo, N.R., Escapa, I.H., Pol, D., and Woodburne, M.O. 2013. Splendid and seldom isolated: The paleobiogeography of Patagonia. Annual Review of Earth and Planetary Sciences, 41:561-603. https://doi.org/10.1146/annurev-earth-050212-124217

Wilf, P., Donovan, M.P., Cúneo, N.R., and Gandolfo, M.A. 2017. The fossil flip-leaves (Retrophyllum, Podocarpaceae) of southern South America. American Journal of Botany, 104:1344-1369. https://doi.org/10.3732/ajb.1700158

Wilf, P., Escapa, I.H., Cúneo, N.R., Kooyman, R.M., Johnson, K.R., and Iglesias, A. 2014. First South American Agathis (Araucariaceae), Eocene of Patagonia. American Journal of Botany, 101:156-179. https://doi.org/10.3732/ajb.1300327

Wing, S.L., Herrera, F., Jaramillo, C.A., Gómez-Navarro, C., Wilf, P., and Labandeira, C.C. 2009. Late Paleocene fossils from the Cerrejón Formation, Colombia, are the earliest record of Neotropical rainforest. Proceedings of the National Academy of Sciences of the United States of America, 106:18627-18632. https://doi.org/10.1073/pnas.0905130106

Woodburne, M.O., Goin, F.J., Bond, M., Carlini, A.A., Gelfo, J.N., López, G.M., Iglesias, A., and Zimicz, A.N. 2014. Paleogene land mammal faunas of South America; a response to global climatic changes and indigenous floral diversity. Journal of Mammalian Evolution, 21:1-73. https://doi.org/10.1007/s10914-012-9222-1 


\section{APPENDIX 1.}

Specimen lists for each angiosperm leaf morphotype by locality, for fossil samples housed in the Paleobotanical Collections of Museo Paleontológico Egidio Feruglio, Trelew, Chubut Province, Argentina (acronym MPEF-Pb). Additional referred specimens are cited by field numbers, which are prefixed by collection locality (Table 1 ) with MPEF-Pb repository number added when assigned. See Table 2 for an inventory of historic type material housed at USNM. (Available in zipped download at https://palaeo-electronica.org/content/2021/3257-patagonia-danian-forests.)

\section{APPENDIX 2.}

Keys to angiosperm leaf/leaflet morphotypes from the Danian Salamanca and Peñas Coloradas formations, San Jorge Basin, Chubut, Argentina. With two organizational pathways, by key numbers and by major leaf shape (subtitles in parentheses). (Available in zipped download at https:// palaeo-electronica.org/content/2021/3257-patagonia-danian-forests.) 